\title{
Nickel-Catalyzed Amidoalkylation Reaction of $\gamma$-Hydroxy \\ Lactams: An Access to 3-Substituted Isoindolinones
}

Shuo Zhang ${ }^{\dagger}$, Xinhua Shi ${ }^{\dagger}$, Jichao Lit, Zitong Hou ${ }^{\dagger}$, Zihe Song ${ }^{\dagger}$, Xiaofeng Sü, Dan Peng ${ }^{* \dagger}$, Feng

Wang $^{\dagger}$, Yitao $\mathrm{Yu}^{\dagger}$, and Guilong Zhao ${ }^{* \dagger}$

${ }_{\dagger}$ Shandong Provincial Key Laboratory for Special Silicone-Containing Materials, Advanced

Materials Institute, Qilu University of Technology (Shandong Academy of Sciences), Jinan 250014, P. R. China

¥Department of Drug Discovery, Guangzhou Lixin Pharmaceuticals, Inc., Guangzhou 510535, P.

R. China

*E-mail: lonarpeng@aliyun.com (D.P.).

*E-mail: zhao_guilong@126.com (G.Z.).

\section{Content}

1. ${ }^{1} \mathrm{H}$ NMR and ${ }^{13} \mathrm{C}$ NMR Spectra. 2-82 


\section{1. ${ }^{1} \mathrm{H}$ NMR and ${ }^{13} \mathrm{C}$ NMR Spectra}

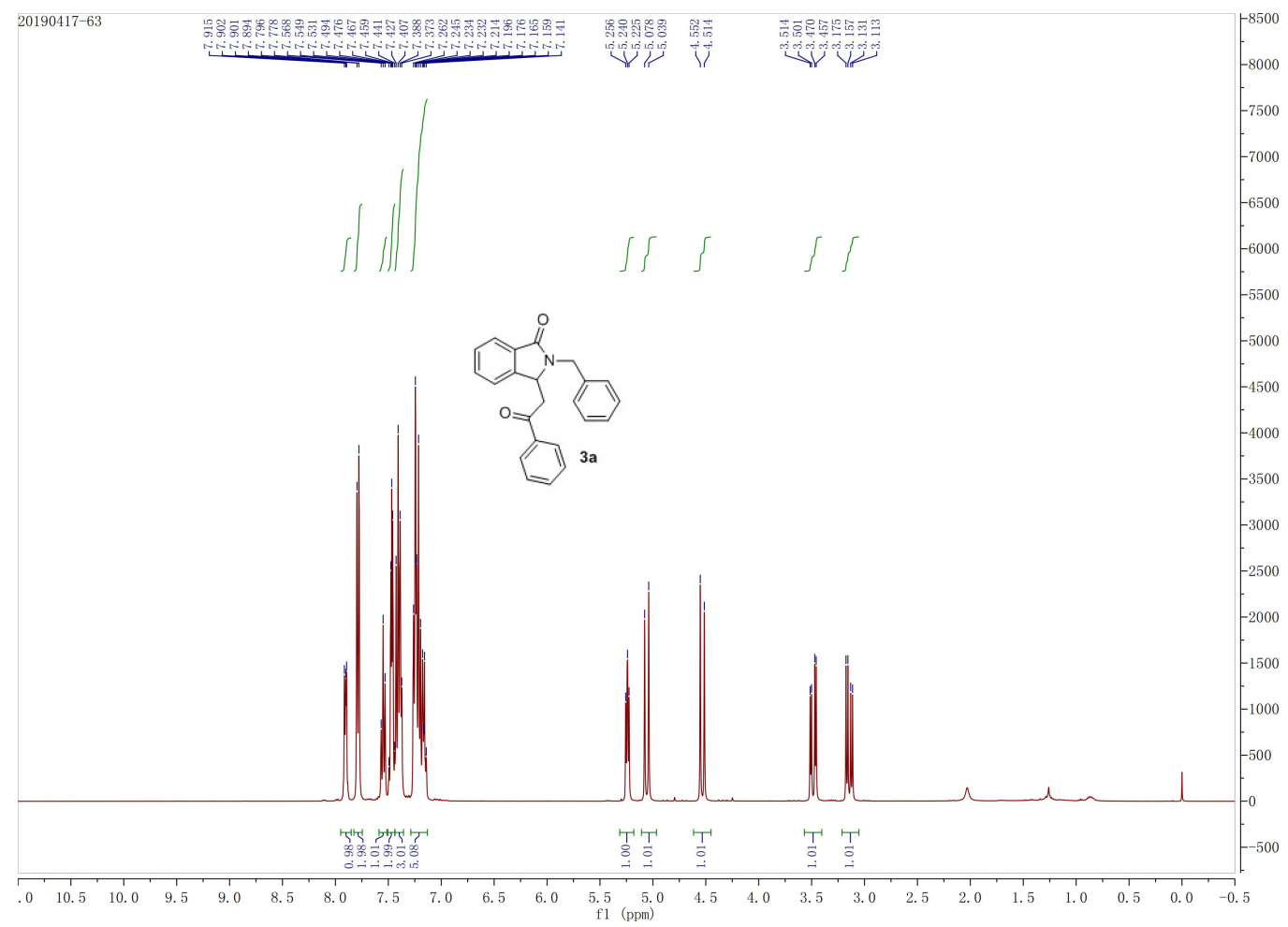

Figure S1. ${ }^{1} \mathrm{HNMR}\left(400 \mathrm{MHz}, \mathrm{CDCl}_{3}\right)$ of compound 3a

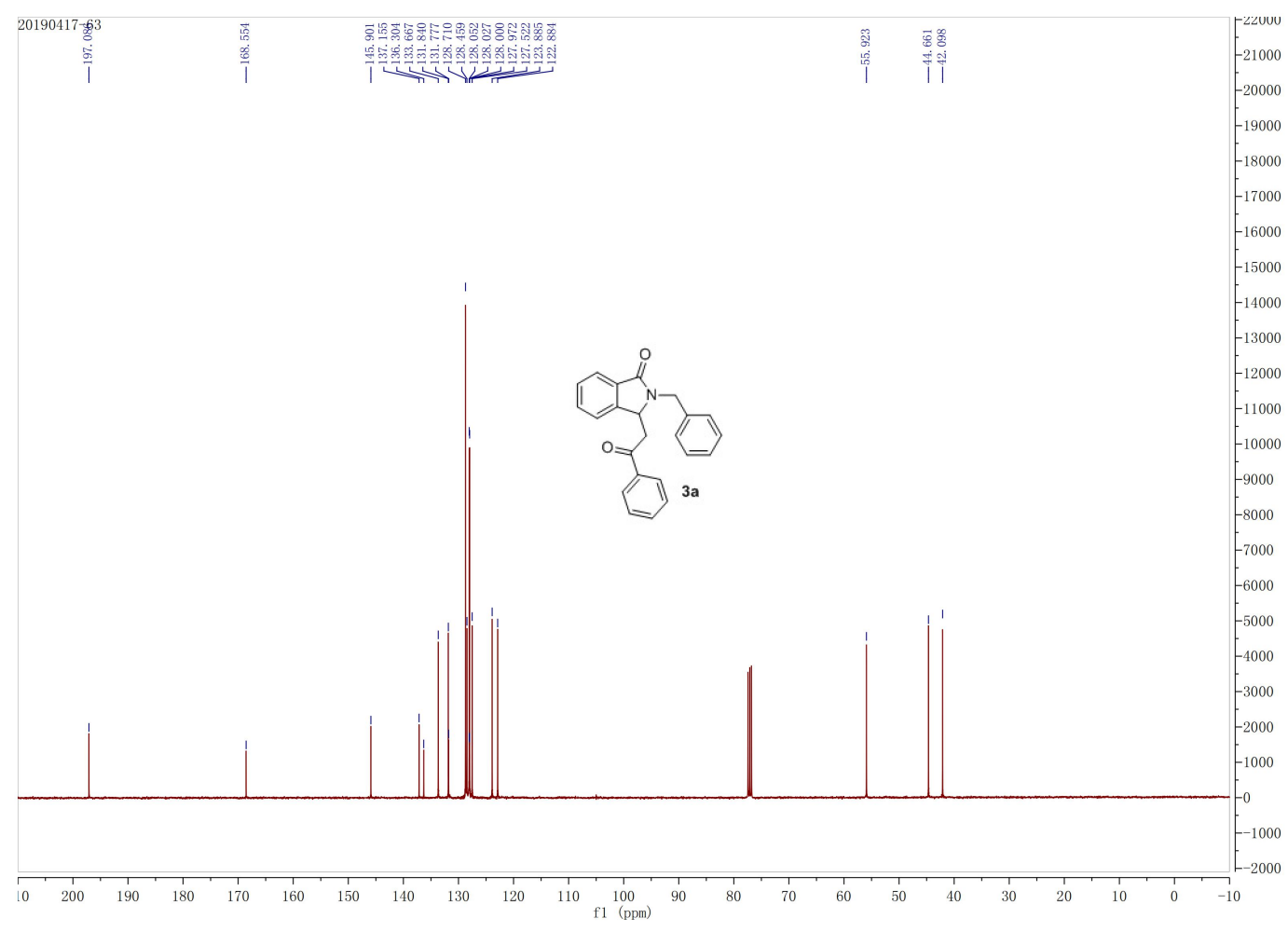

Figure S2. ${ }^{13} \mathrm{CNMR}\left(100 \mathrm{MHz}, \mathrm{CDCl}_{3}\right)$ of compound 3a 


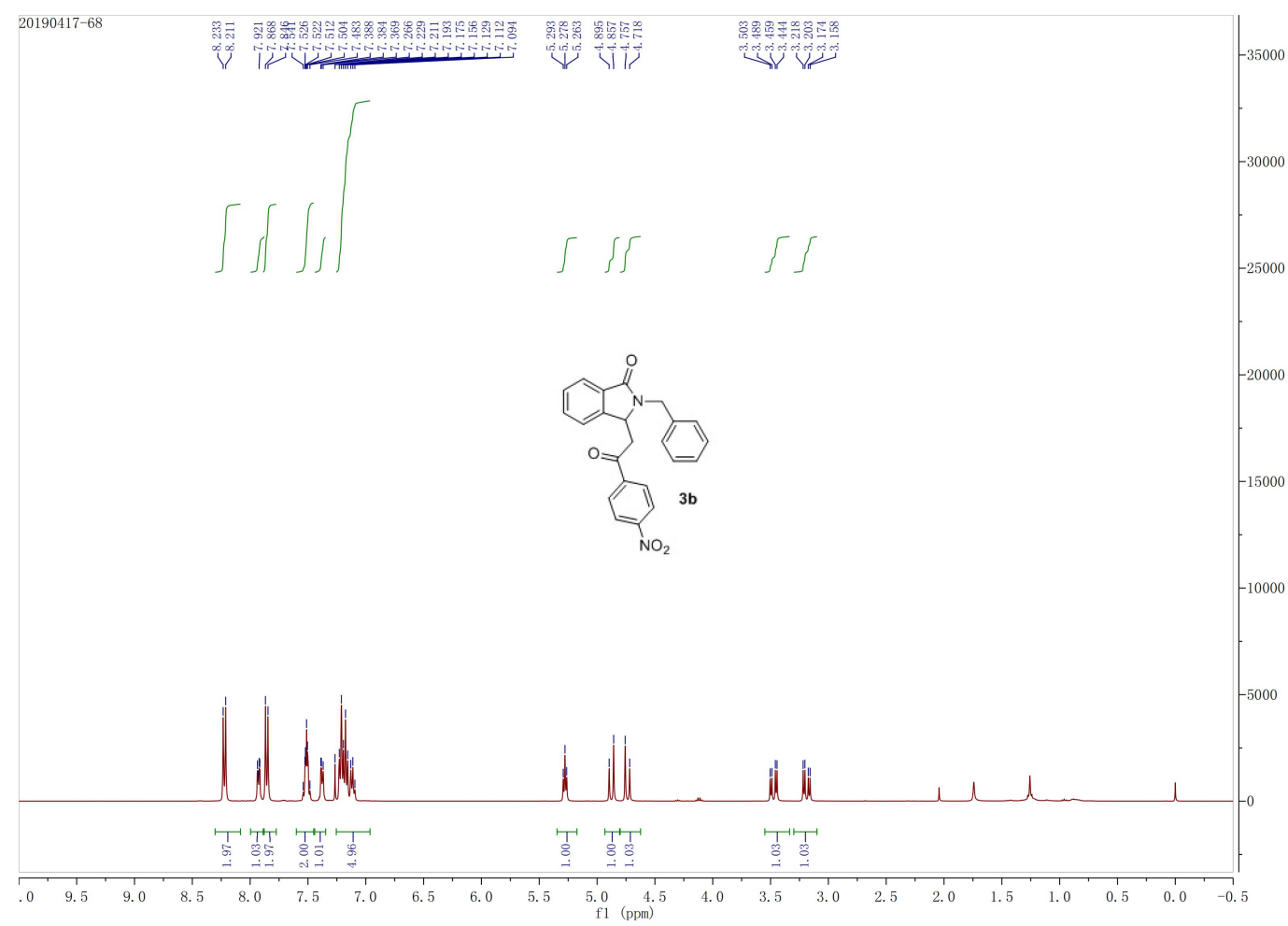

Figure S3. ${ }^{1} \mathrm{HNMR}\left(400 \mathrm{MHz}, \mathrm{CDCl}_{3}\right.$ ) of compound 3b

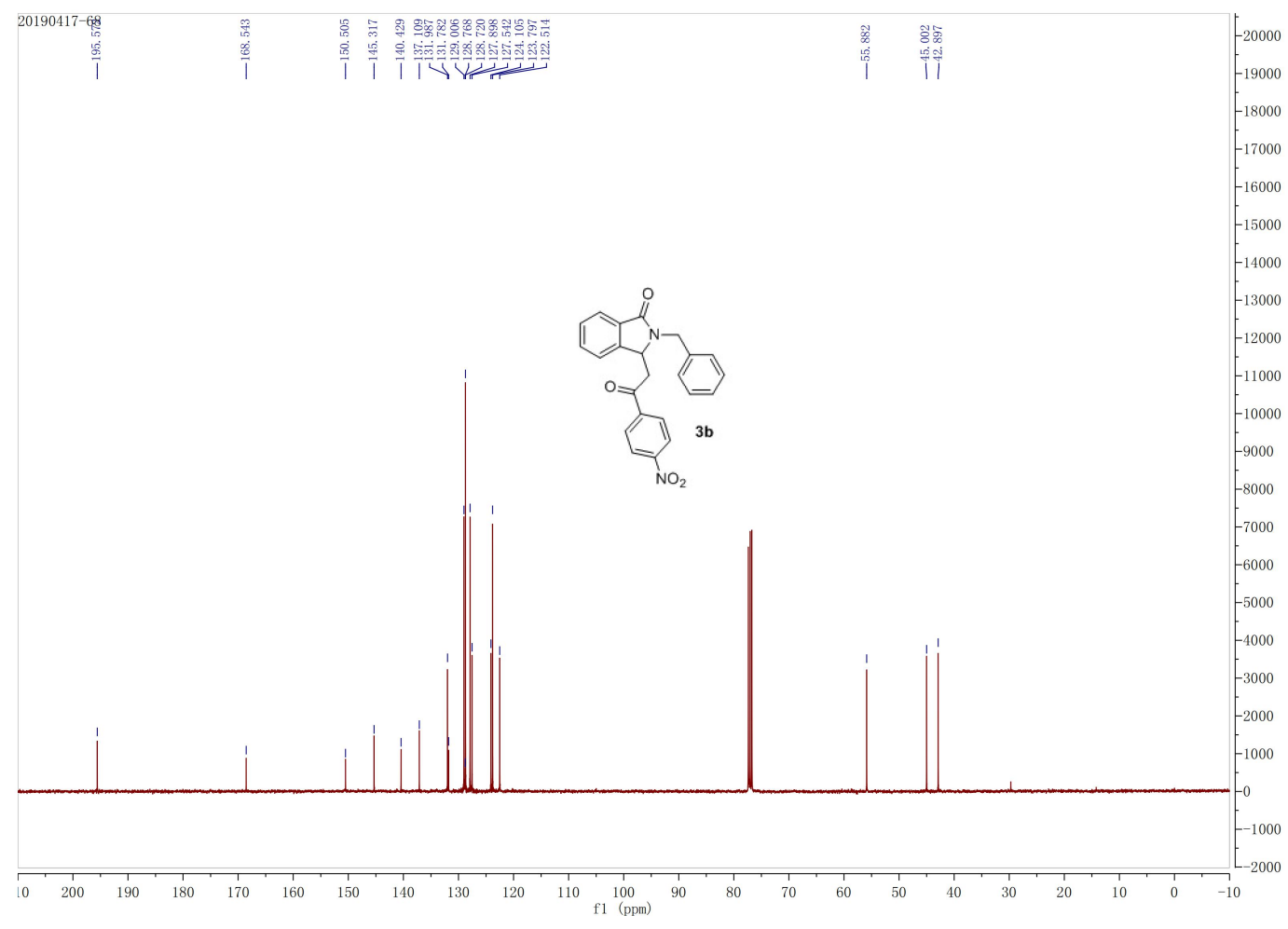

Figure S4. ${ }^{13} \mathrm{CNMR}\left(100 \mathrm{MHz}, \mathrm{CDCl}_{3}\right)$ of compound $3 \mathrm{~b}$ 


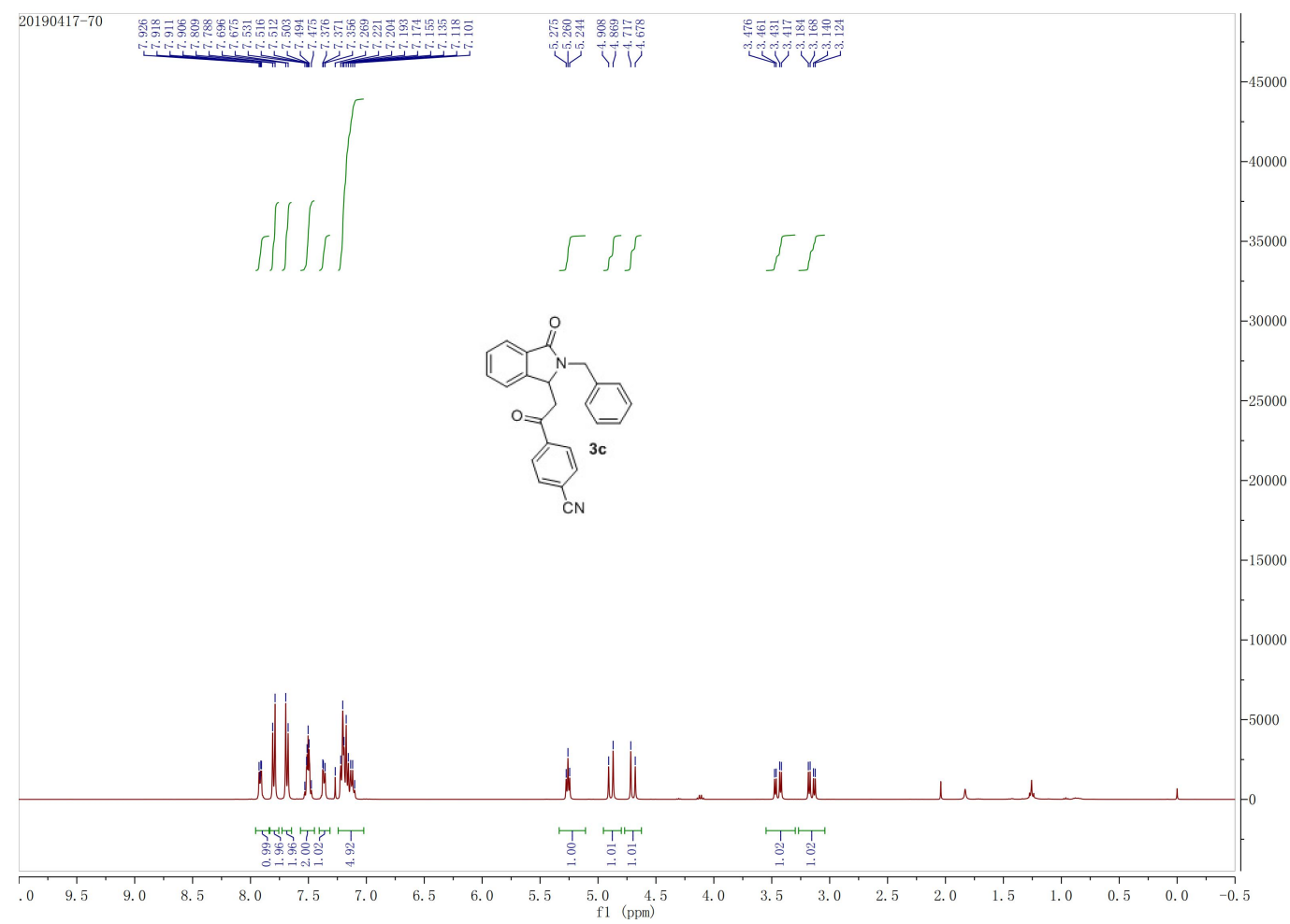

Figure S5. ${ }^{1} \mathrm{HNMR}\left(400 \mathrm{MHz}, \mathrm{CDCl}_{3}\right)$ of compound $3 \mathrm{c}$

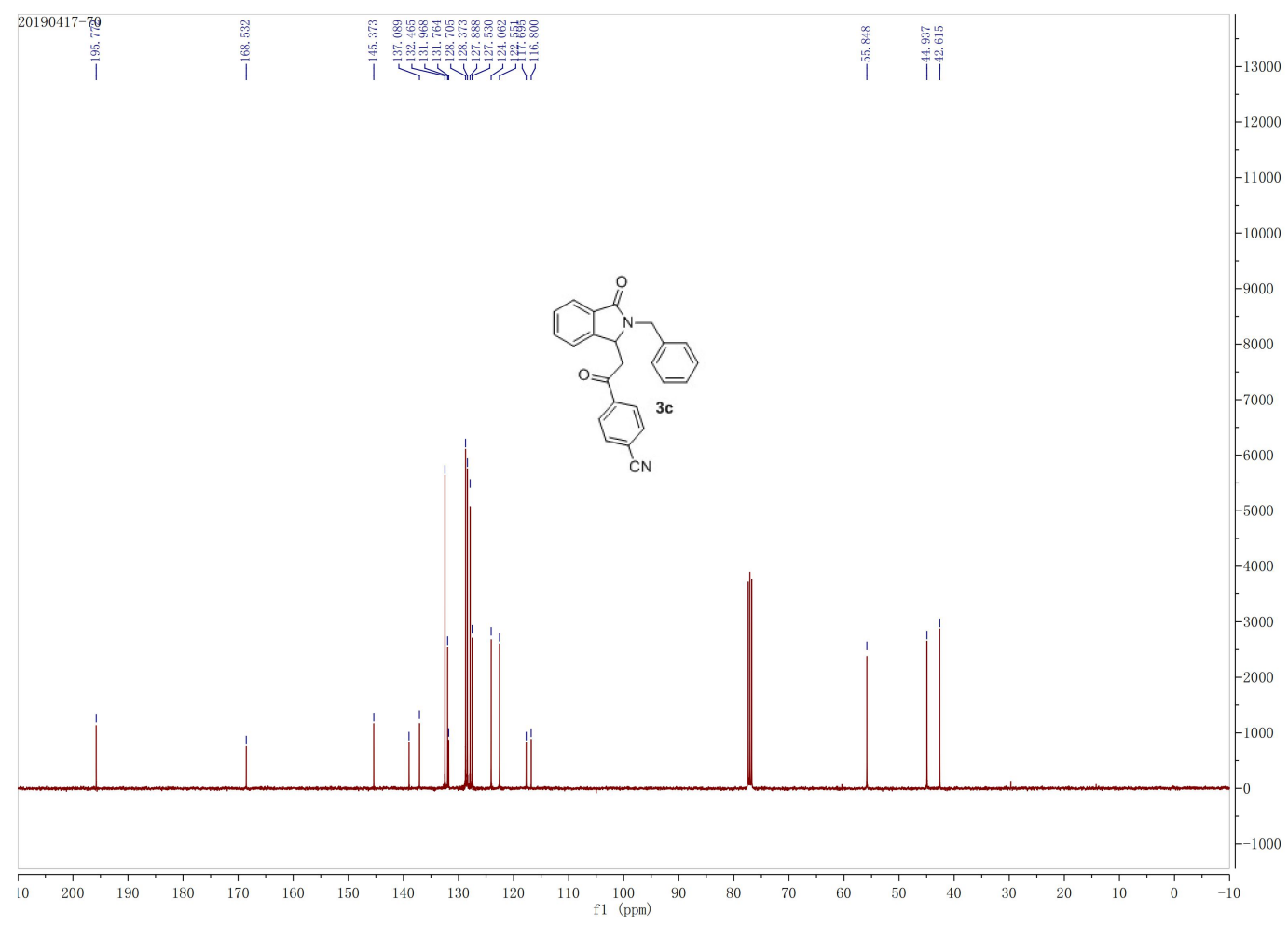

Figure S6. ${ }^{13} \mathrm{CNMR}\left(100 \mathrm{MHz}, \mathrm{CDCl}_{3}\right)$ of compound 3c 


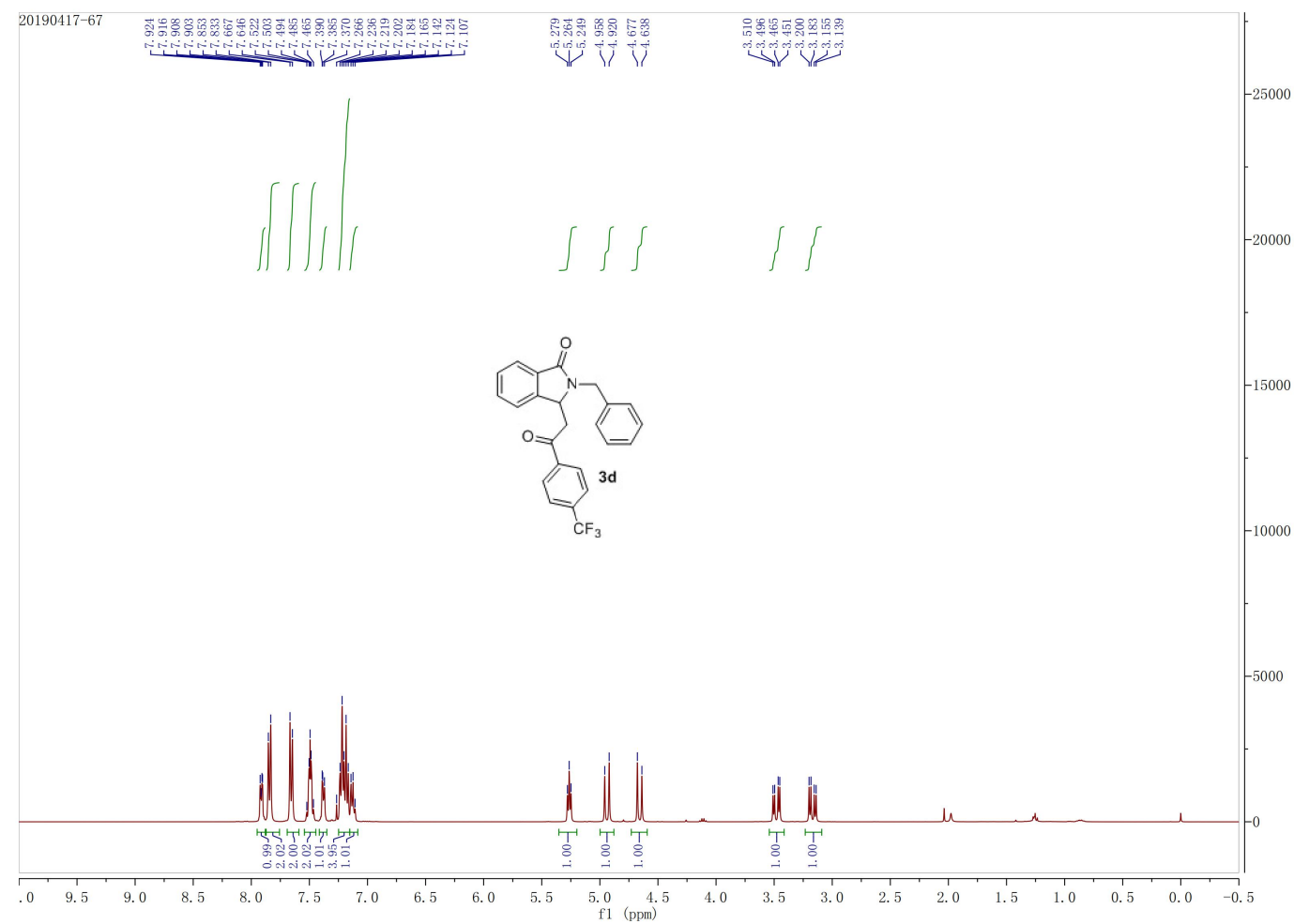

Figure S7. ${ }^{1} \mathrm{HNMR}\left(400 \mathrm{MHz}, \mathrm{CDCl}_{3}\right)$ of compound 3d

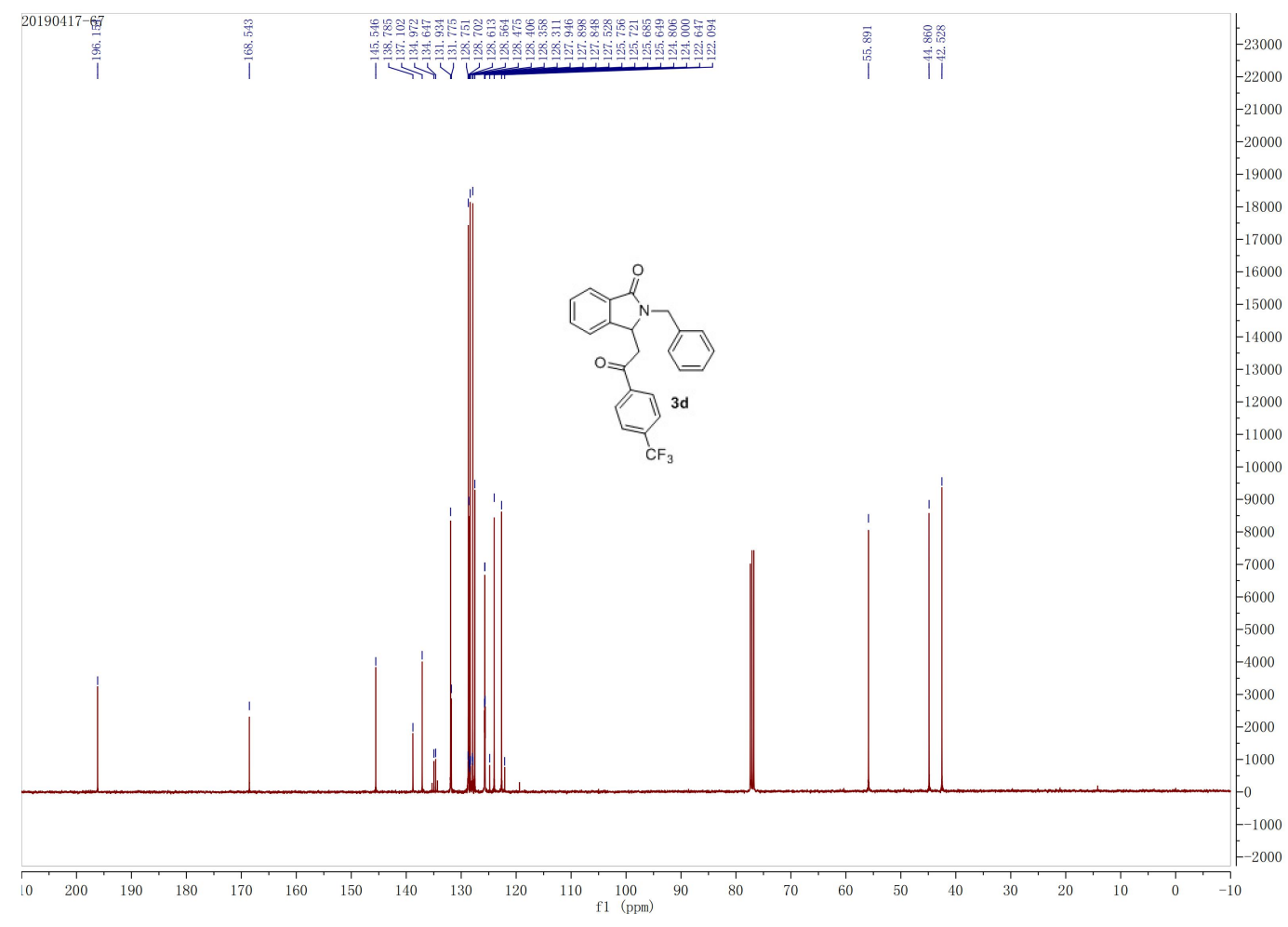

Figure S8. ${ }^{13} \mathrm{CNMR}\left(100 \mathrm{MHz}, \mathrm{CDCl}_{3}\right)$ of compound 3d 


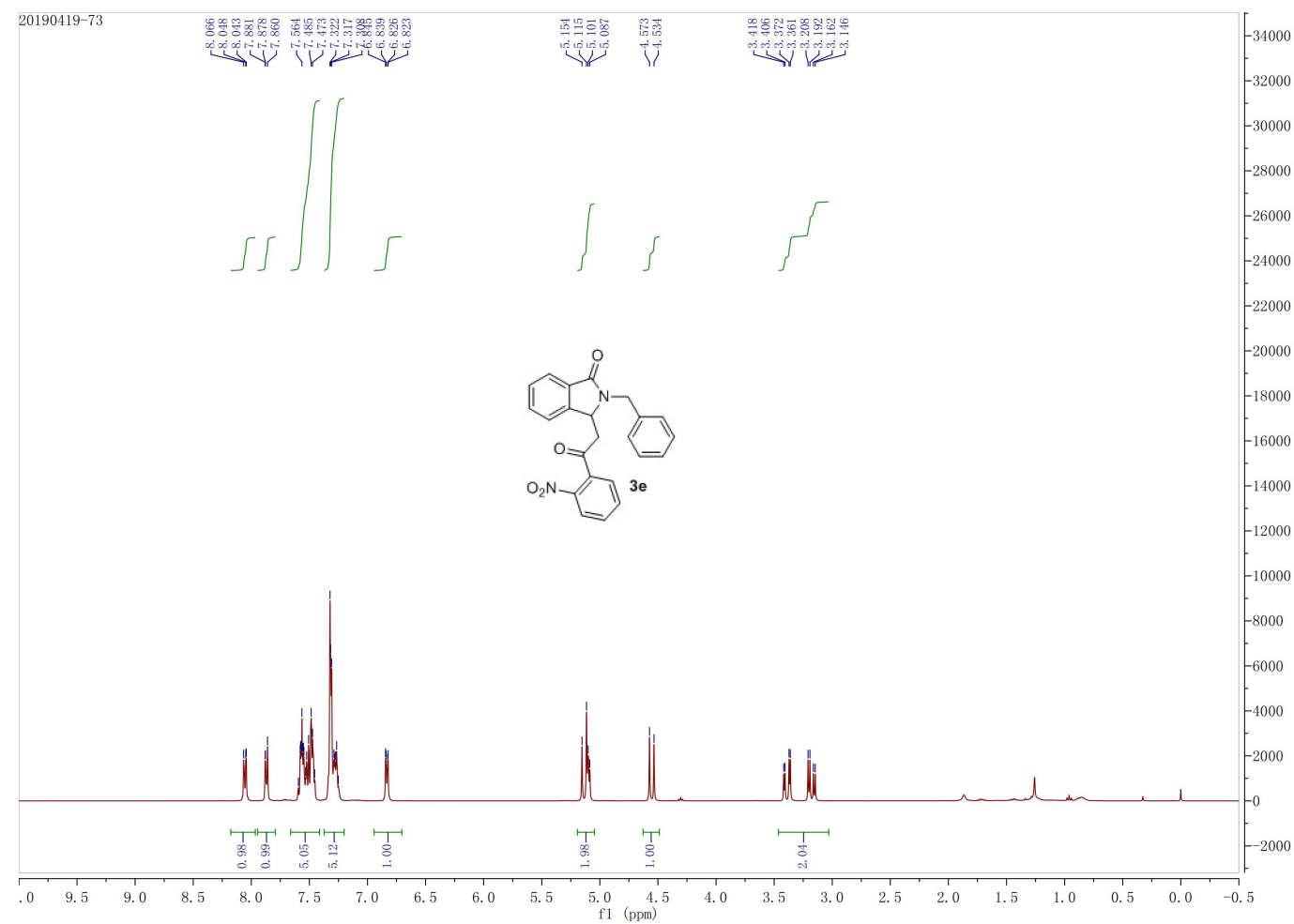

Figure S9. ${ }^{1} \mathrm{HNMR}\left(400 \mathrm{MHz}, \mathrm{CDCl}_{3}\right)$ of compound $3 \mathrm{e}$

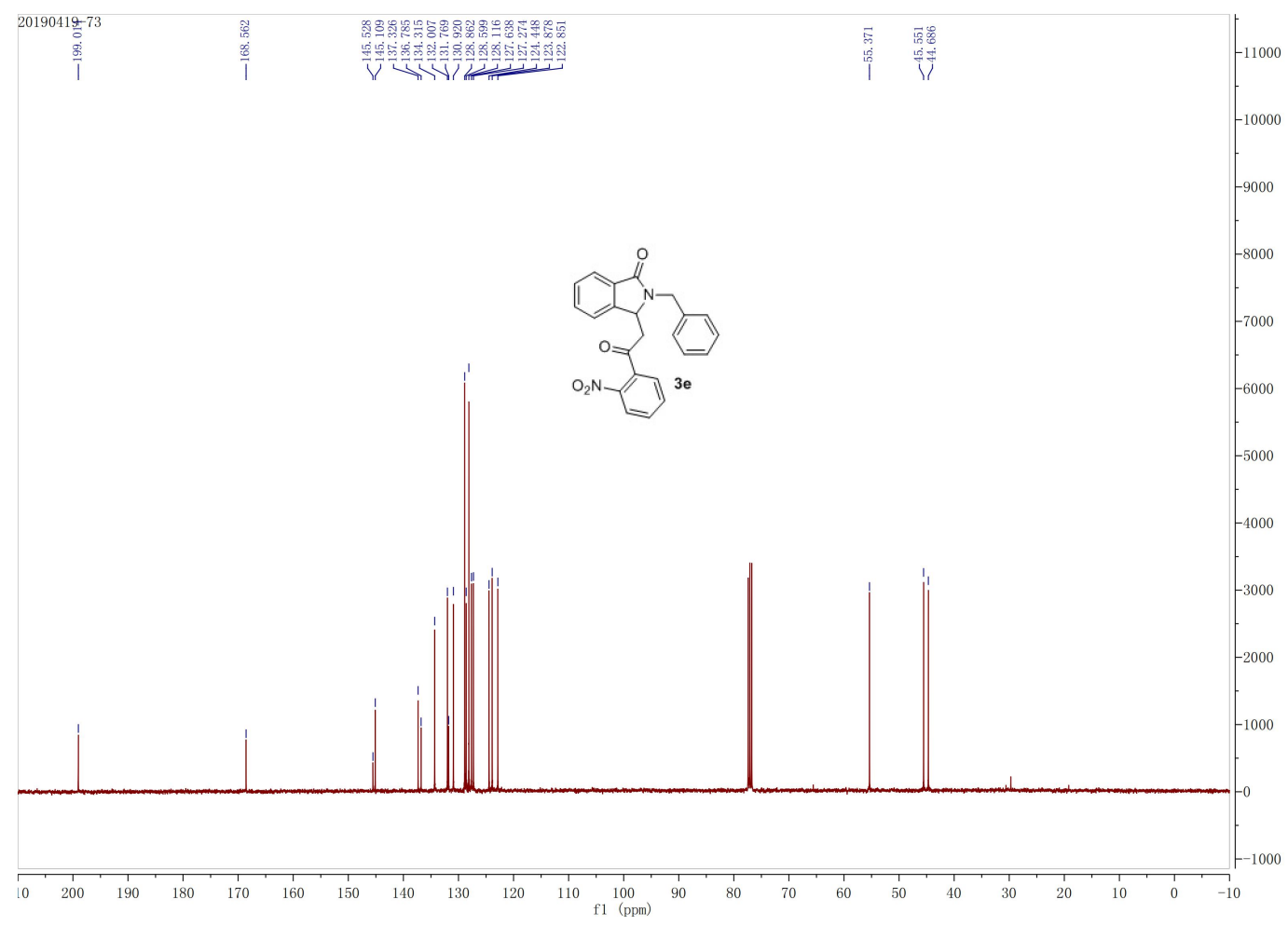

Figure S10. ${ }^{13} \mathrm{CNMR}\left(100 \mathrm{MHz}, \mathrm{CDCl}_{3}\right)$ of compound $3 \mathrm{e}$ 


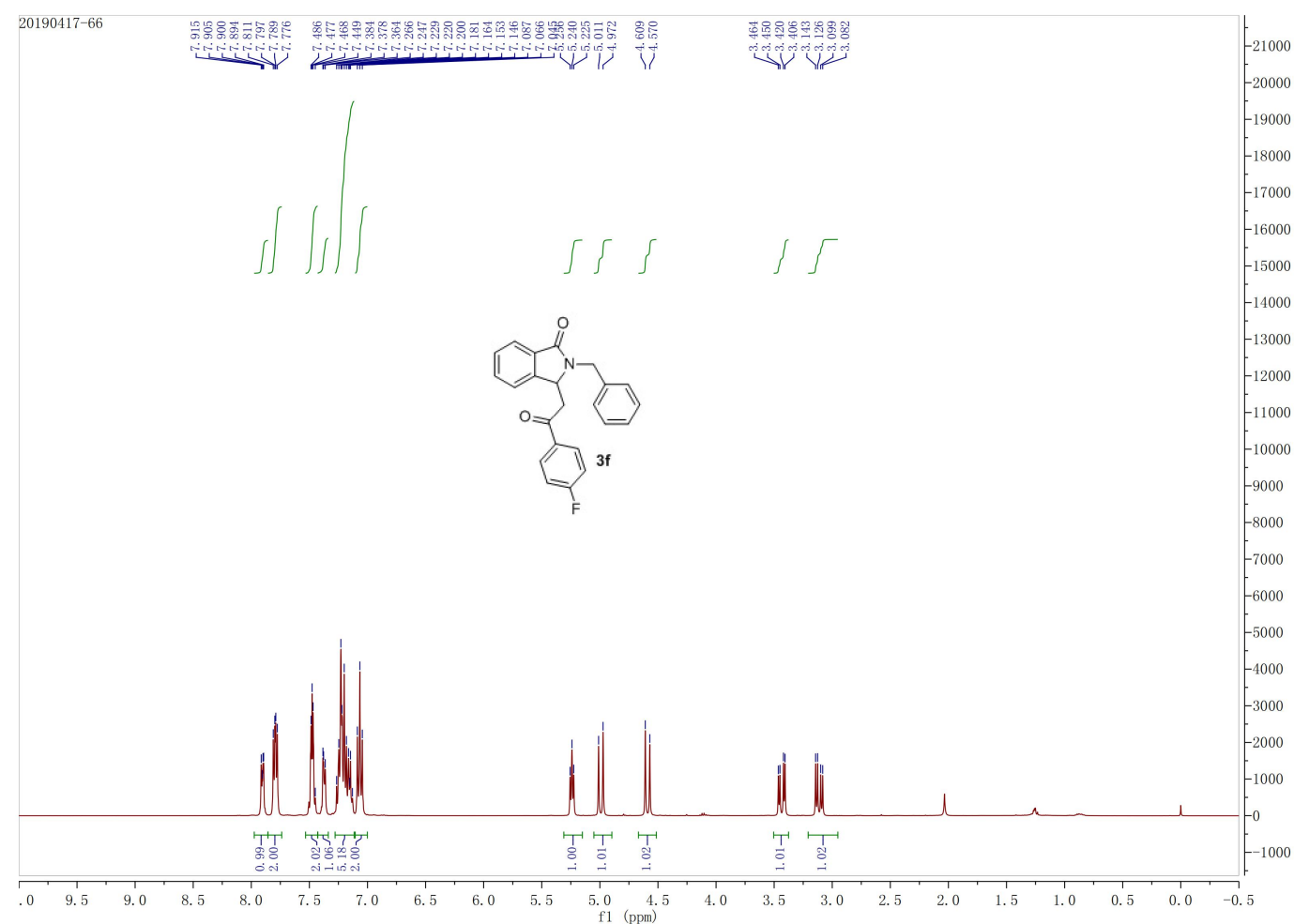

Figure S11. ${ }^{1} \mathrm{HNMR}\left(400 \mathrm{MHz}, \mathrm{CDCl}_{3}\right)$ of compound $3 \mathrm{f}$

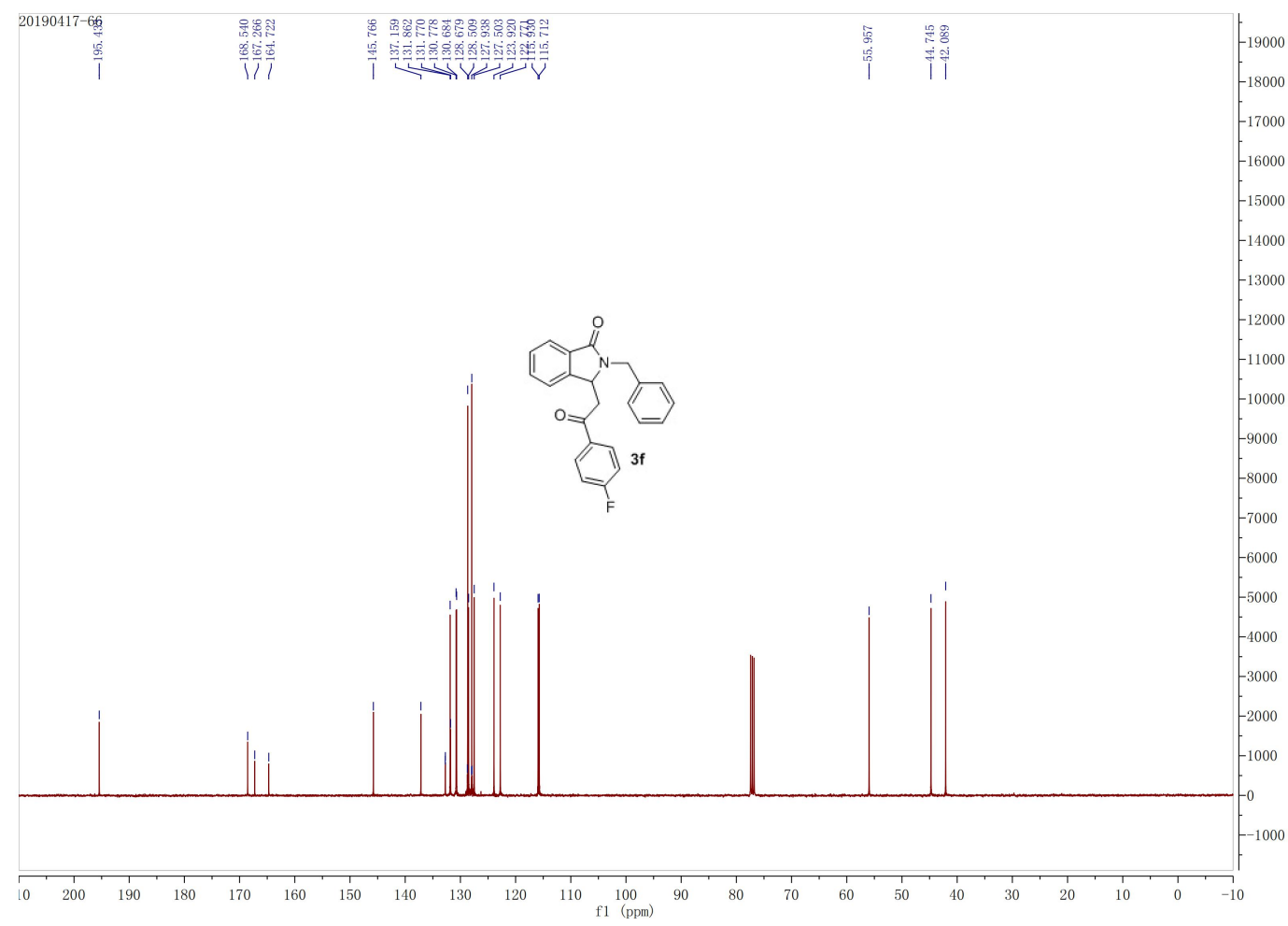

Figure S12. ${ }^{13} \mathrm{CNMR}\left(100 \mathrm{MHz}, \mathrm{CDCl}_{3}\right)$ of compound $3 \mathrm{f}$ 


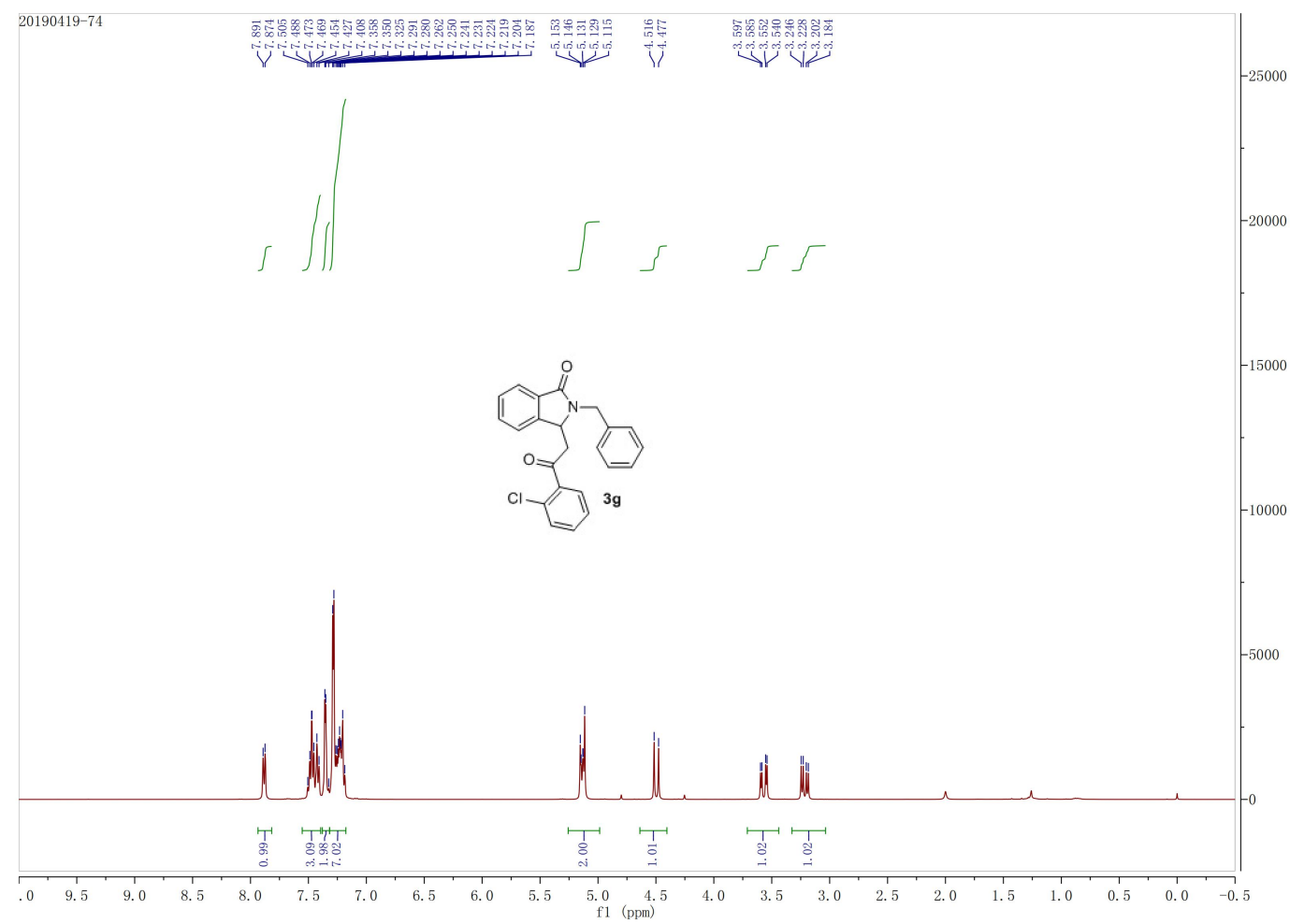

Figure S13. ${ }^{1} \mathrm{HNMR}\left(400 \mathrm{MHz}, \mathrm{CDCl}_{3}\right)$ of compound $3 \mathrm{~g}$

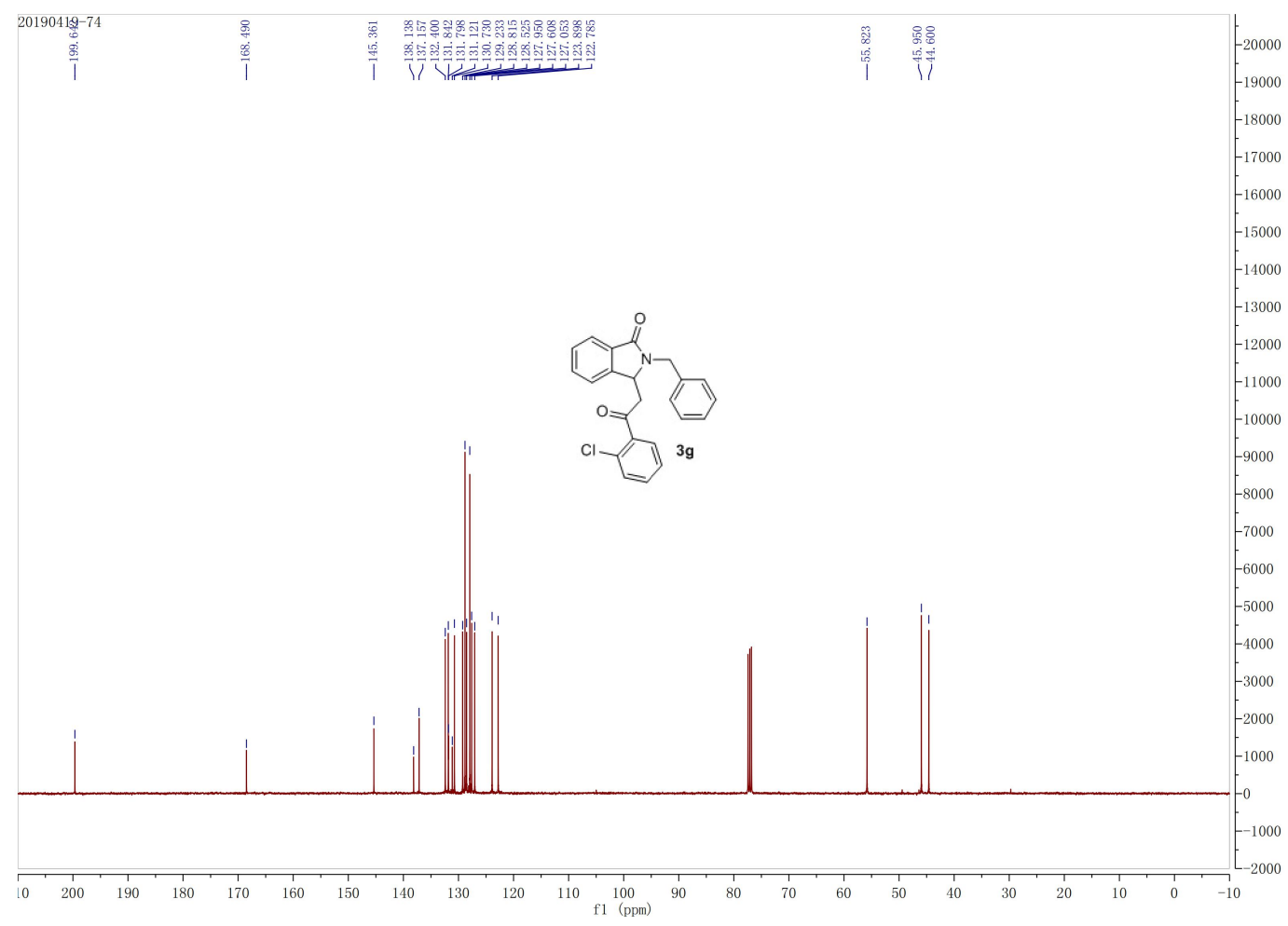

Figure S14. ${ }^{13} \mathrm{CNMR}\left(100 \mathrm{MHz}, \mathrm{CDCl}_{3}\right)$ of compound $3 \mathrm{~g}$ 


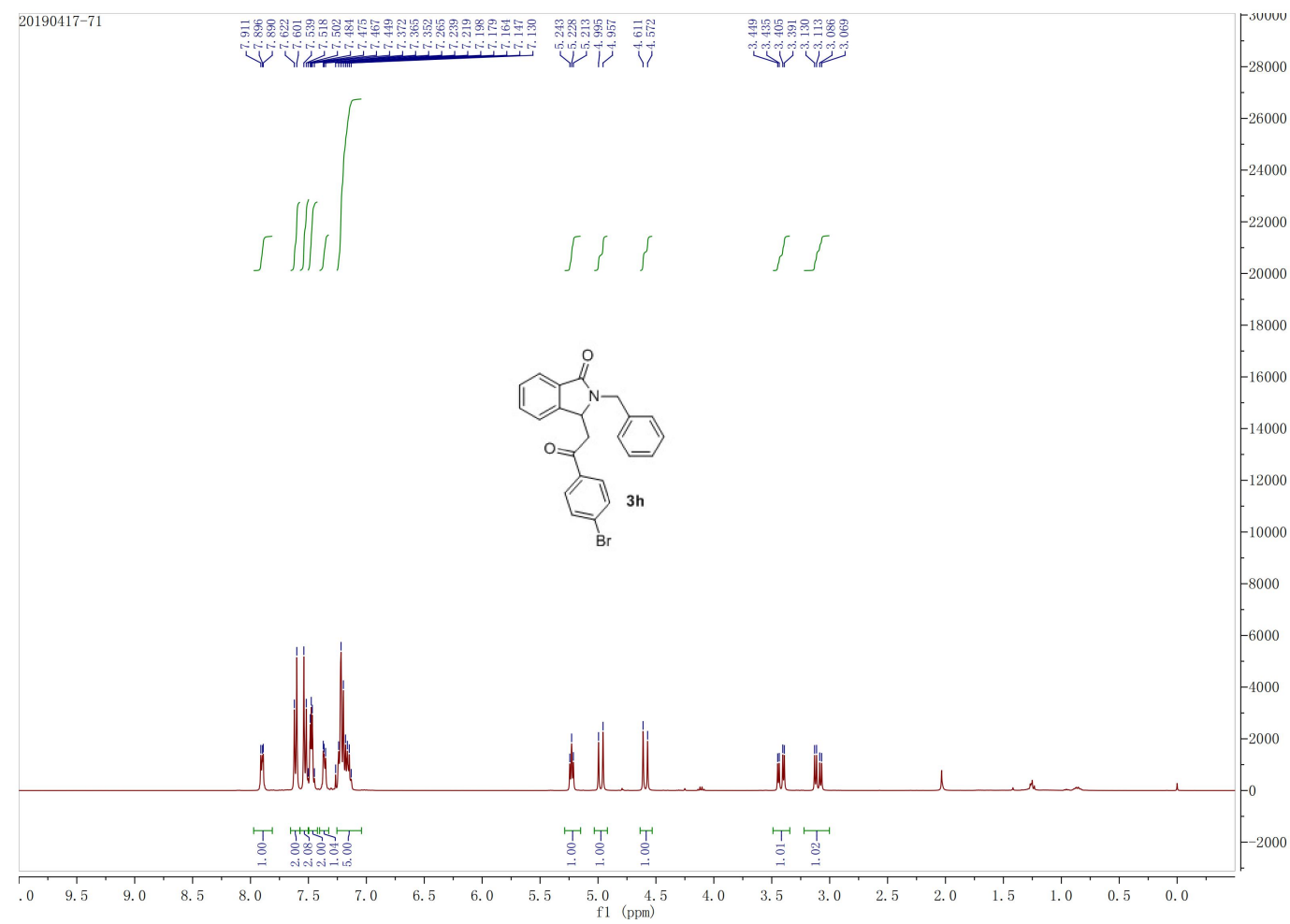

Figure S15. ${ }^{1} \mathrm{HNMR}\left(400 \mathrm{MHz}, \mathrm{CDCl}_{3}\right)$ of compound $3 \mathrm{~h}$

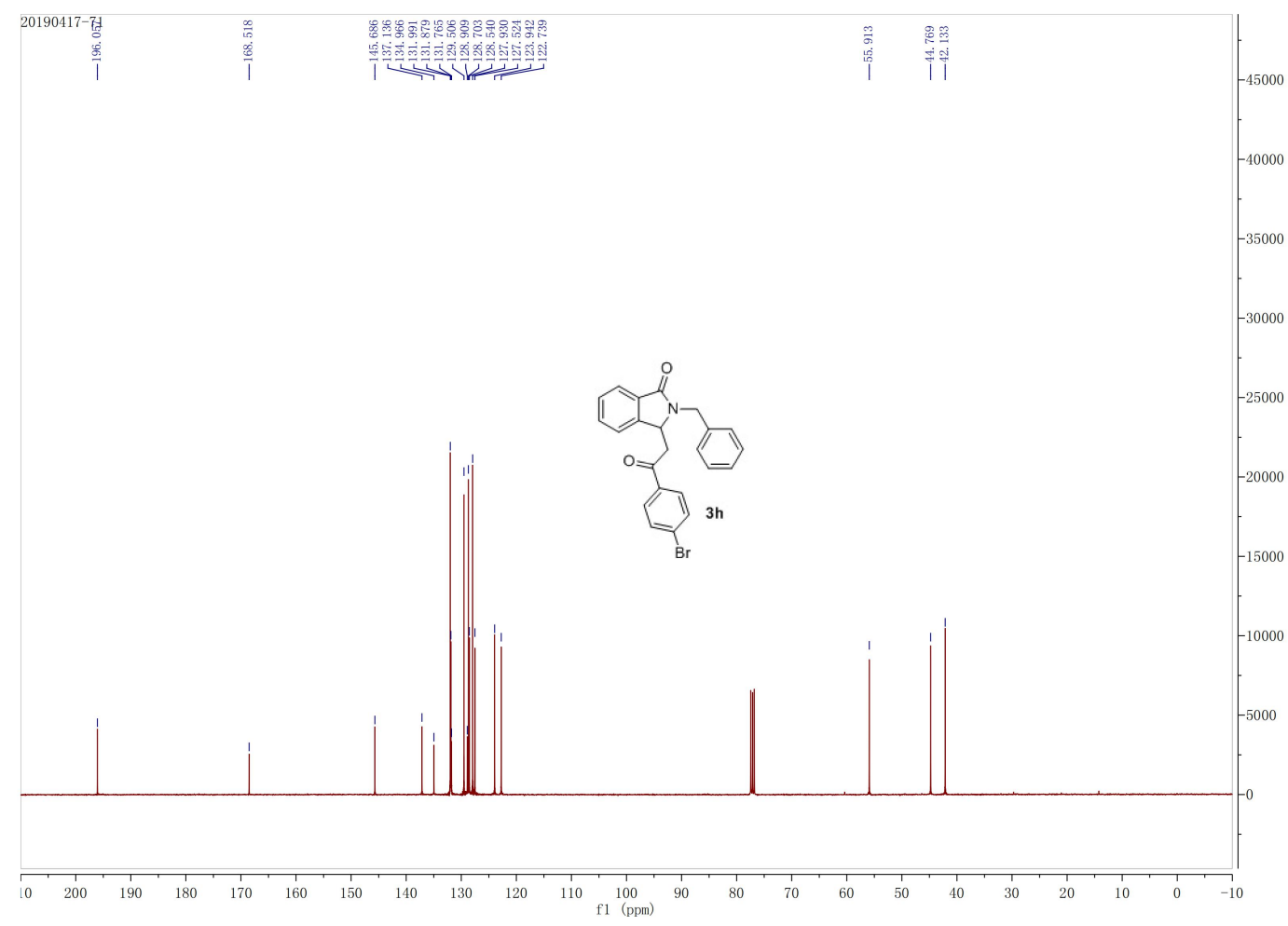

Figure S16. ${ }^{13} \mathrm{CNMR}\left(100 \mathrm{MHz}, \mathrm{CDCl}_{3}\right)$ of compound $3 \mathrm{~h}$ 


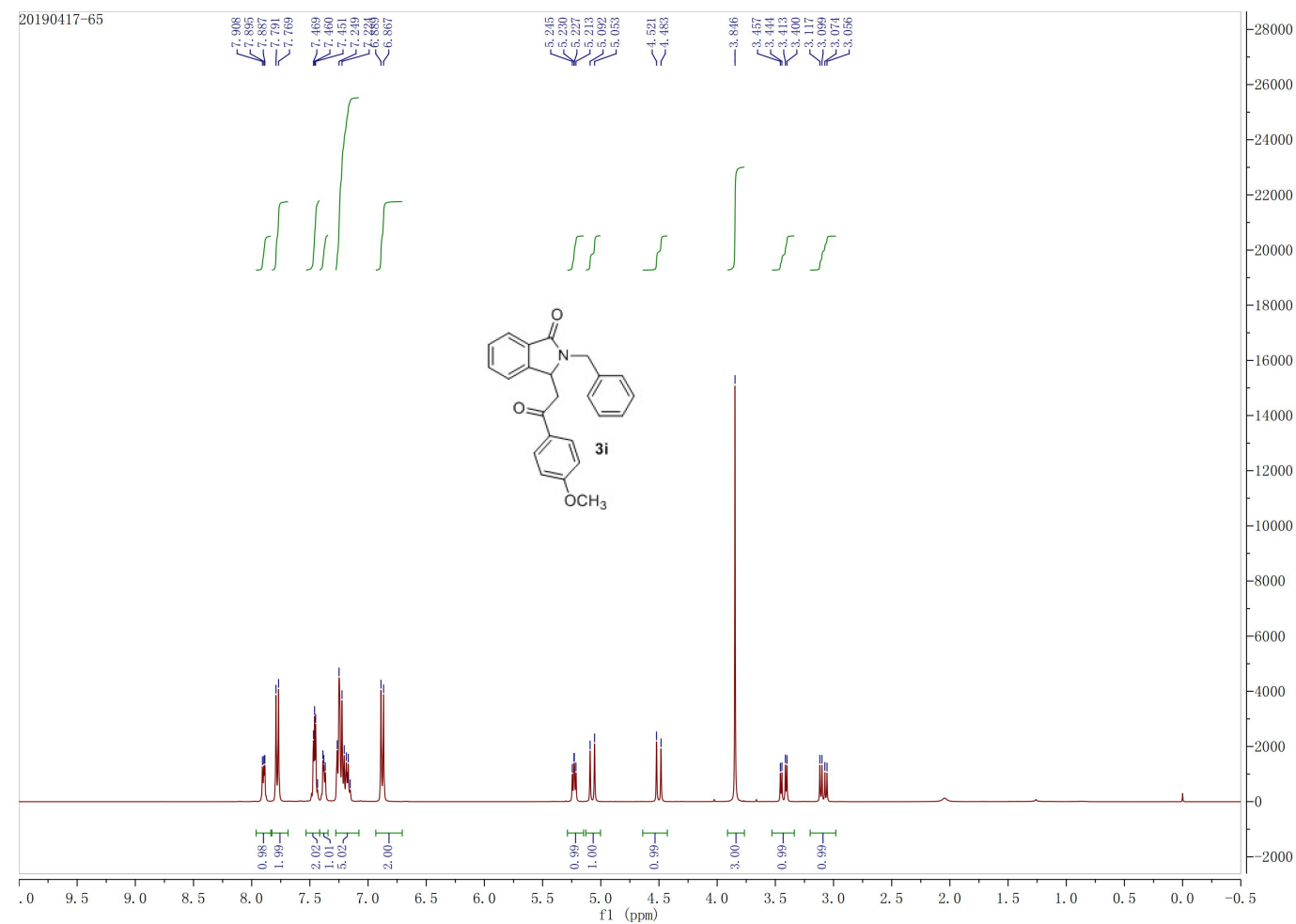

Figure S17. ${ }^{1} \mathrm{HNMR}\left(400 \mathrm{MHz}, \mathrm{CDCl}_{3}\right)$ of compound $3 \mathrm{i}$

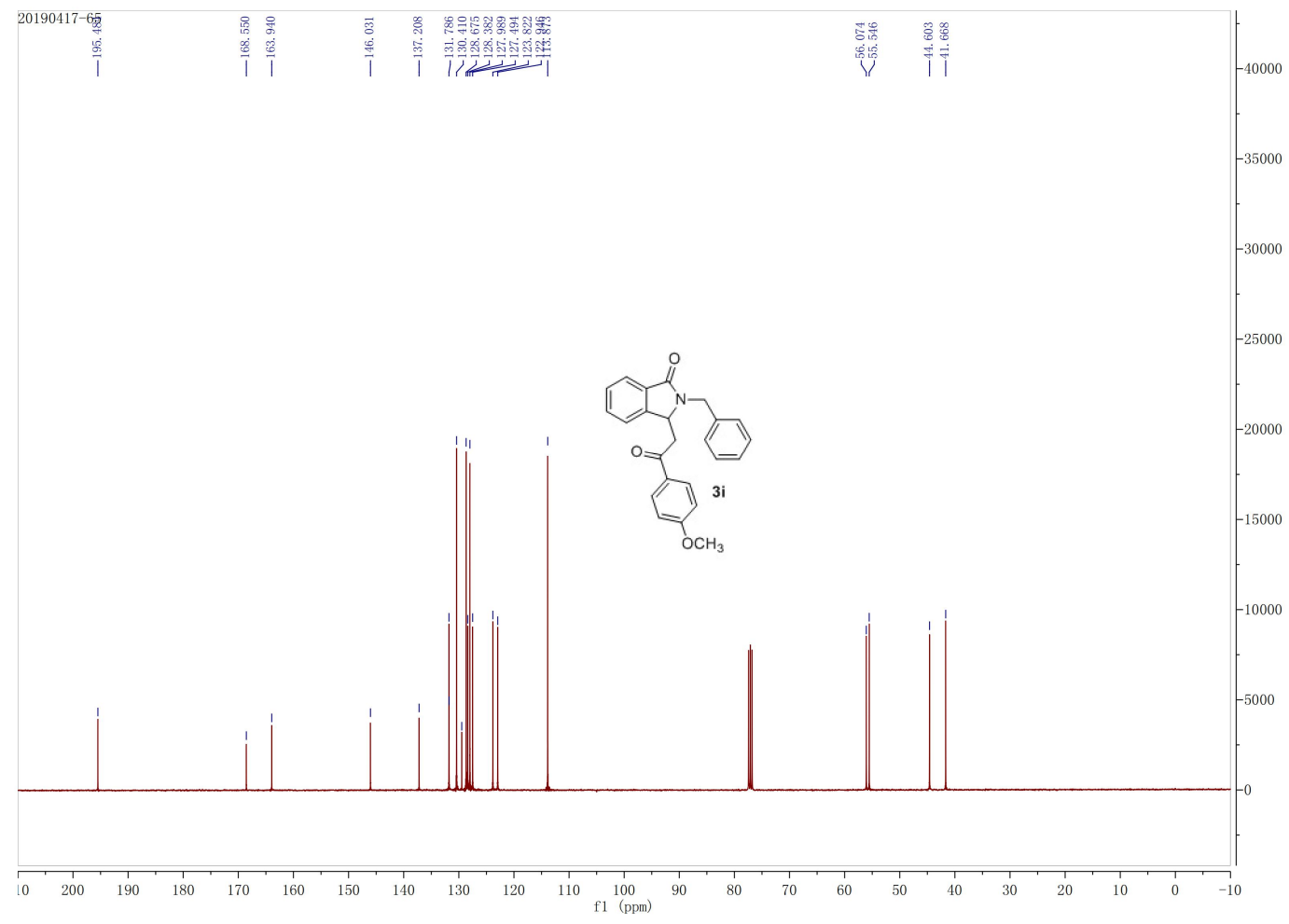

Figure S18. ${ }^{13} \mathrm{CNMR}\left(100 \mathrm{MHz}, \mathrm{CDCl}_{3}\right)$ of compound 3i 


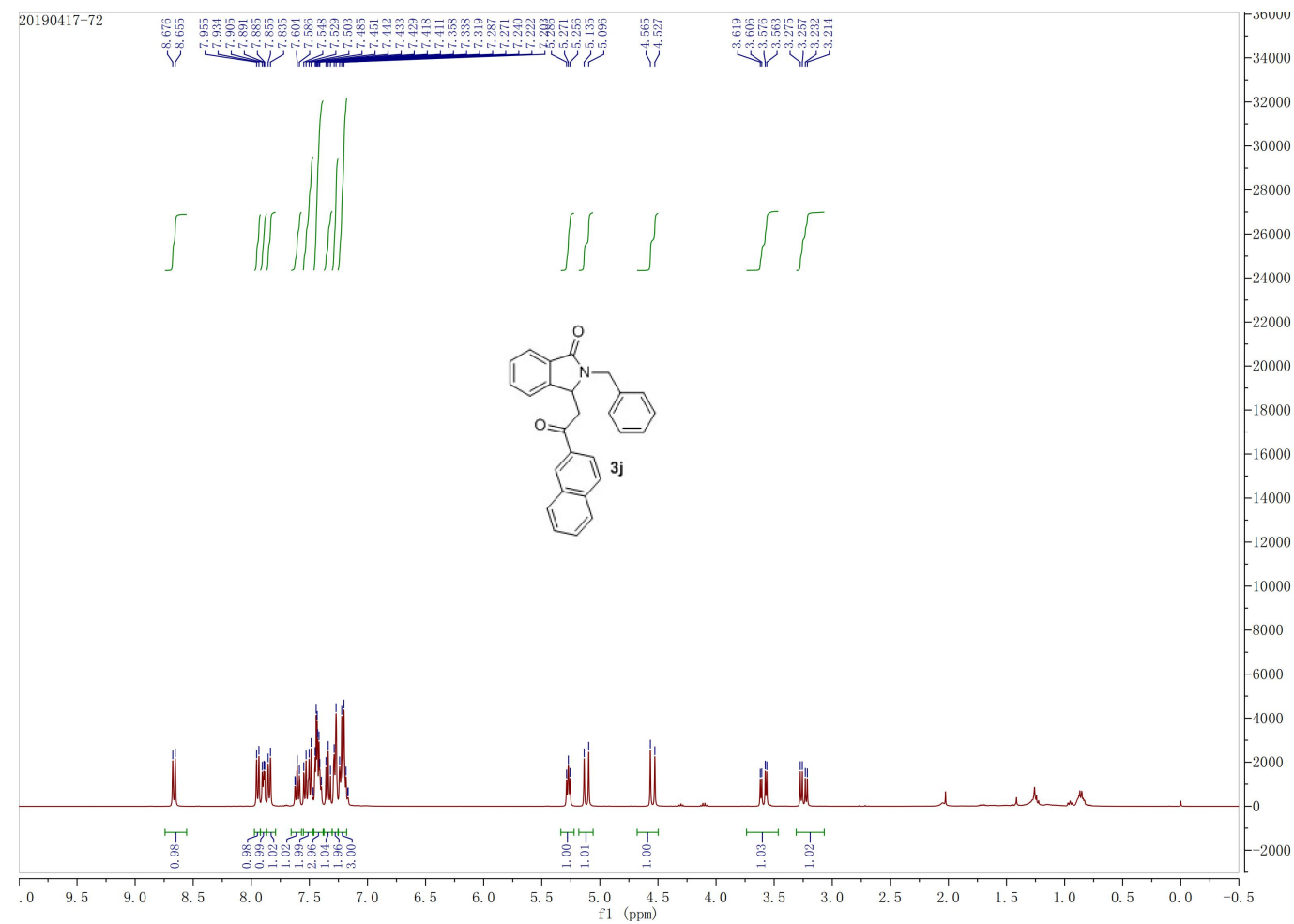

Figure S19. ${ }^{1} \mathrm{HNMR}\left(400 \mathrm{MHz}, \mathrm{CDCl}_{3}\right)$ of compound $3 \mathrm{j}$

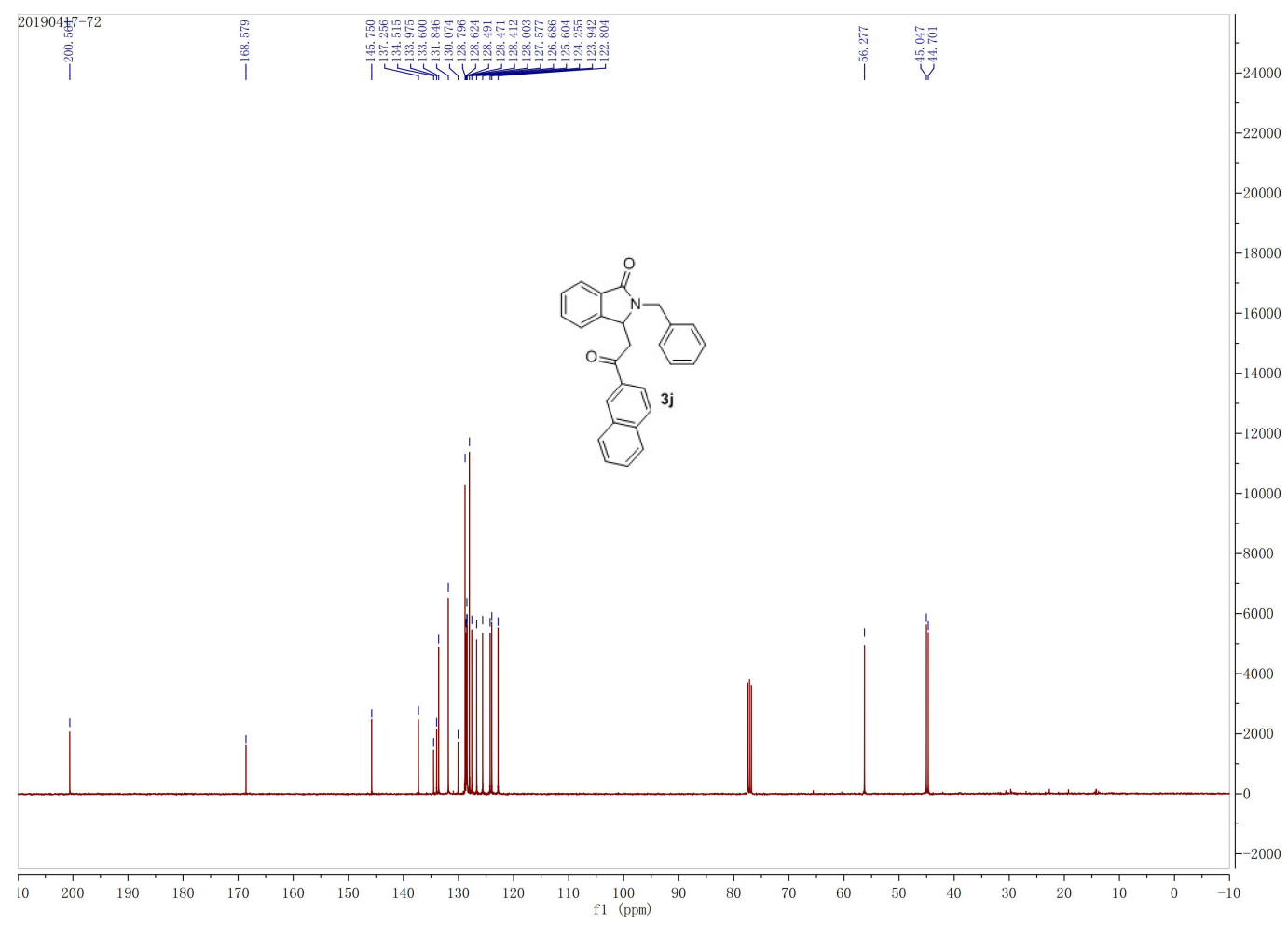

Figure S20. ${ }^{13} \mathrm{CNMR}\left(100 \mathrm{MHz}, \mathrm{CDCl}_{3}\right)$ of compound $3 \mathrm{j}$ 


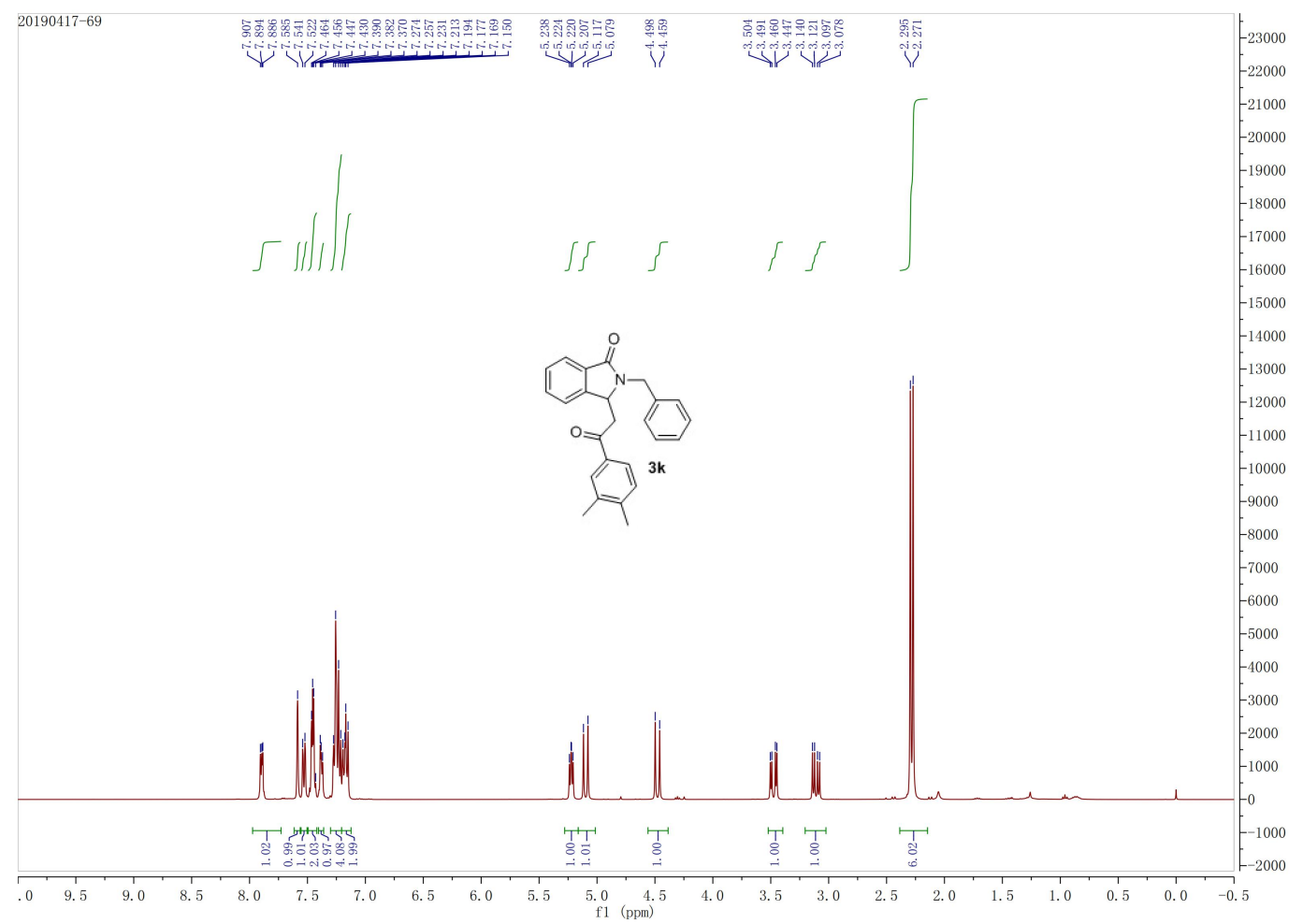

Figure S21. ${ }^{1} \mathrm{HNMR}\left(400 \mathrm{MHz}, \mathrm{CDCl}_{3}\right)$ of compound $3 \mathrm{k}$

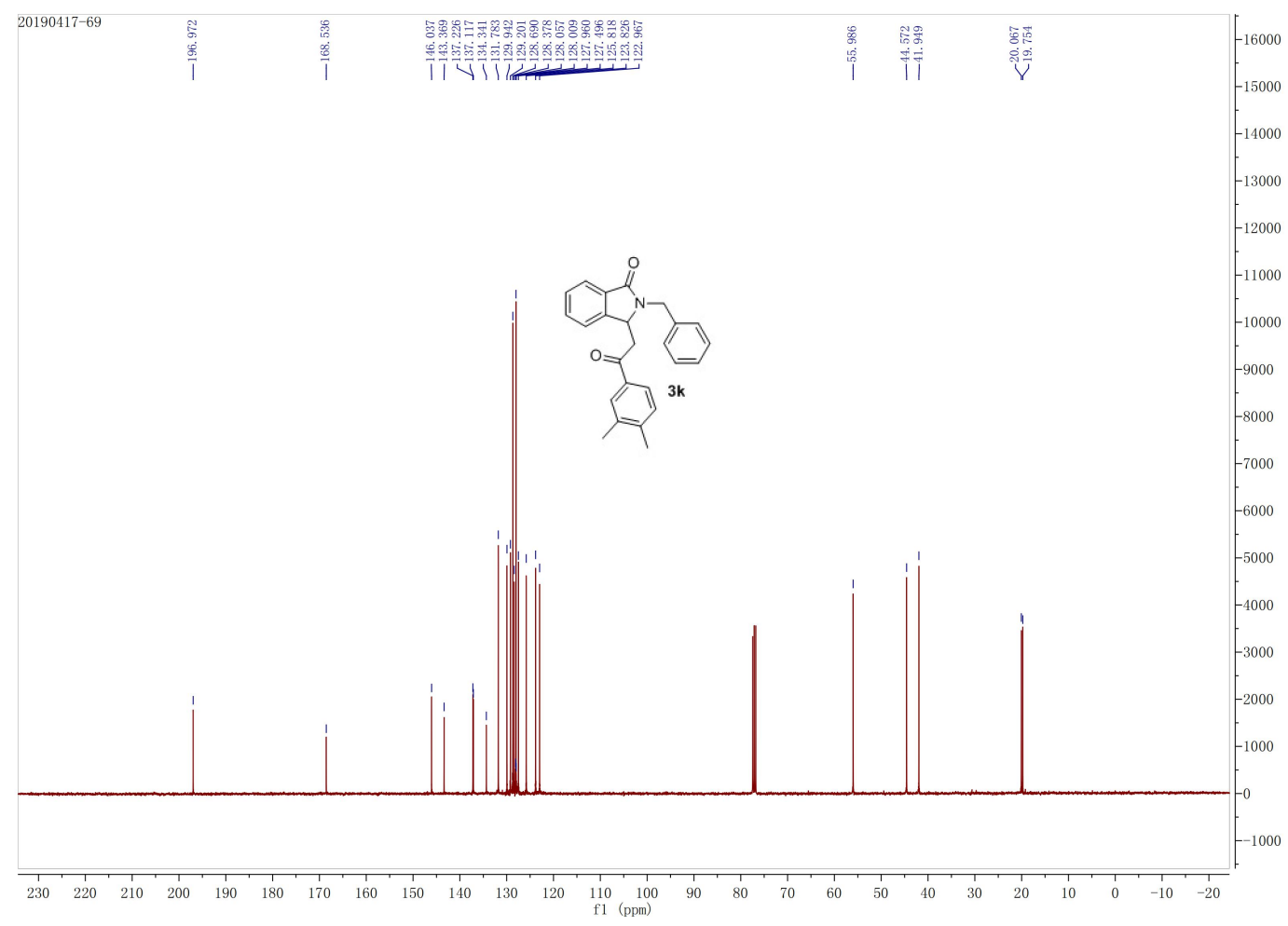

Figure S22. ${ }^{13} \mathrm{CNMR}\left(100 \mathrm{MHz}, \mathrm{CDCl}_{3}\right)$ of compound 3k 


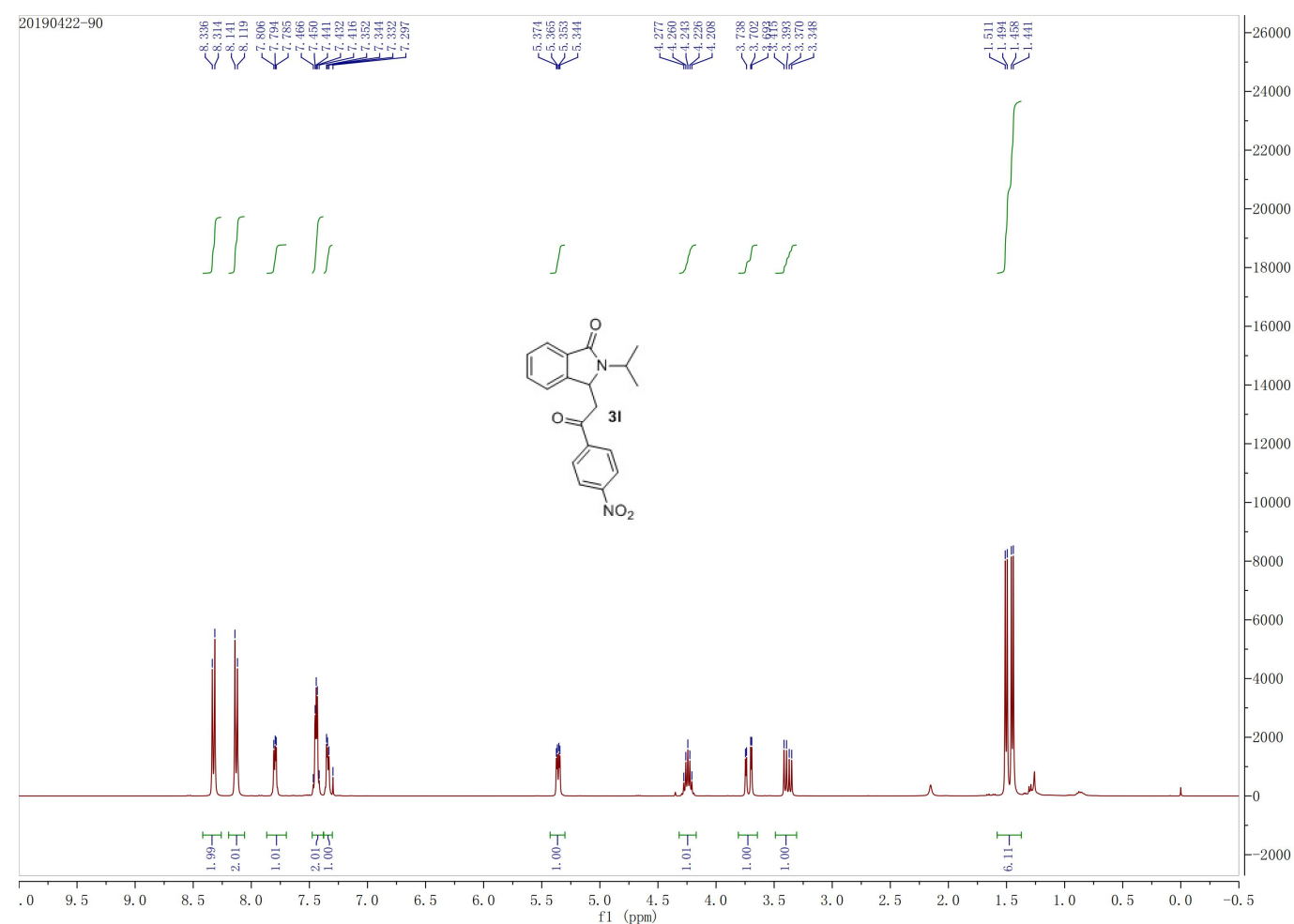

Figure S23. ${ }^{1} \mathrm{HNMR}\left(400 \mathrm{MHz}, \mathrm{CDCl}_{3}\right)$ of compound $3 \mathrm{I}$

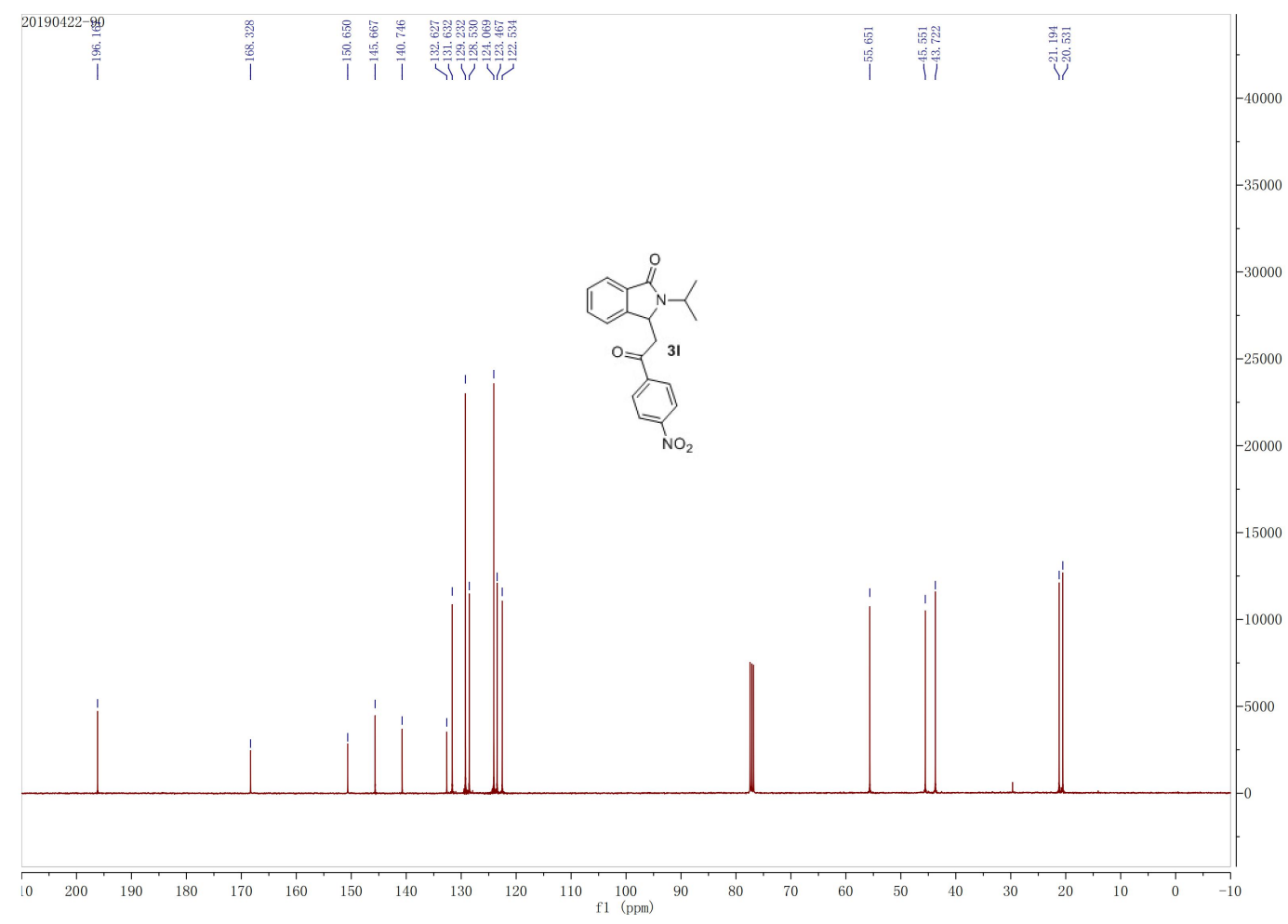

Figure S24. ${ }^{13} \mathrm{CNMR}\left(100 \mathrm{MHz}, \mathrm{CDCl}_{3}\right)$ of compound 31 


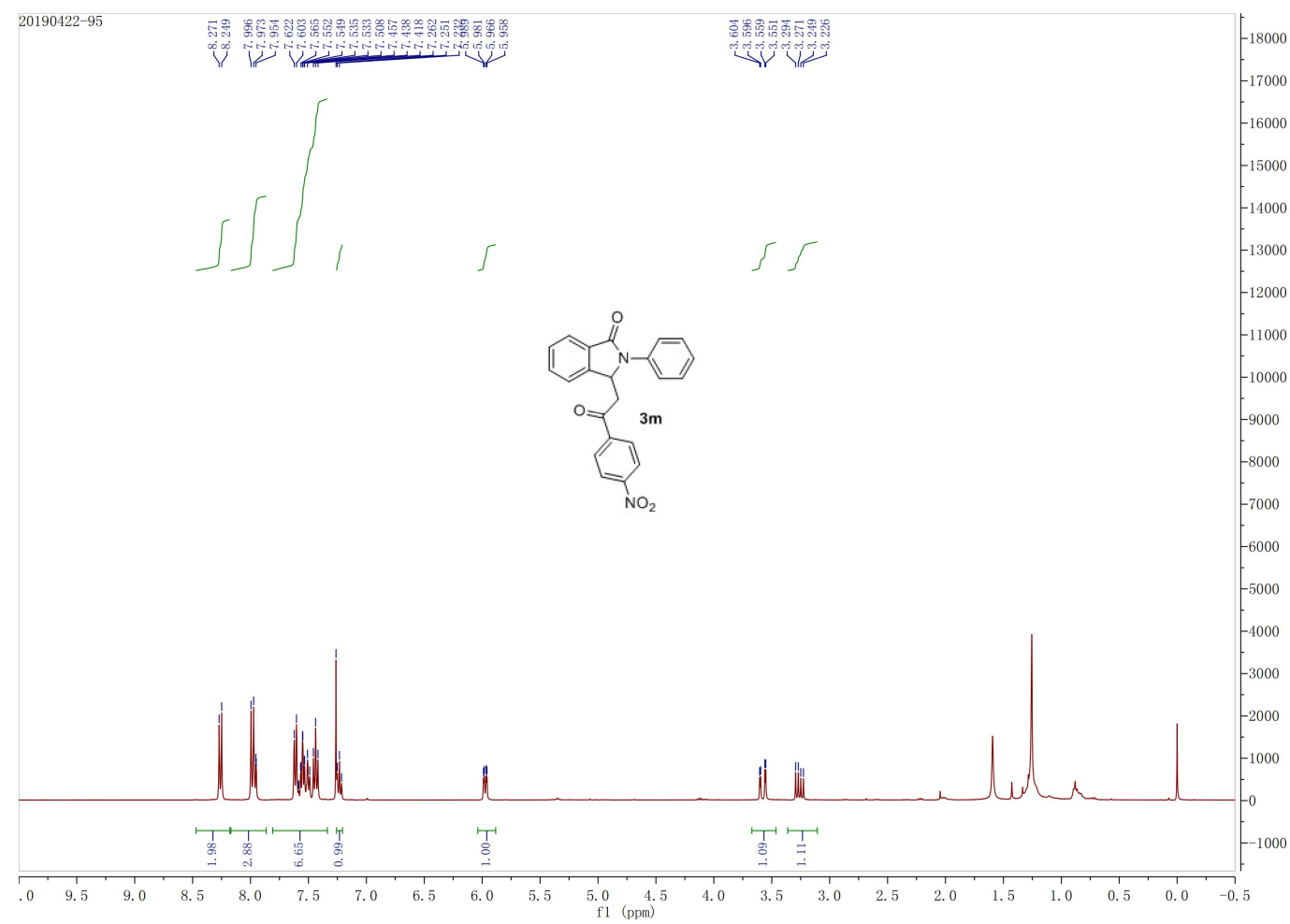

Figure S25. ${ }^{1} \mathrm{HNMR}\left(400 \mathrm{MHz}, \mathrm{CDCl}_{3}\right.$ ) of compound 3m

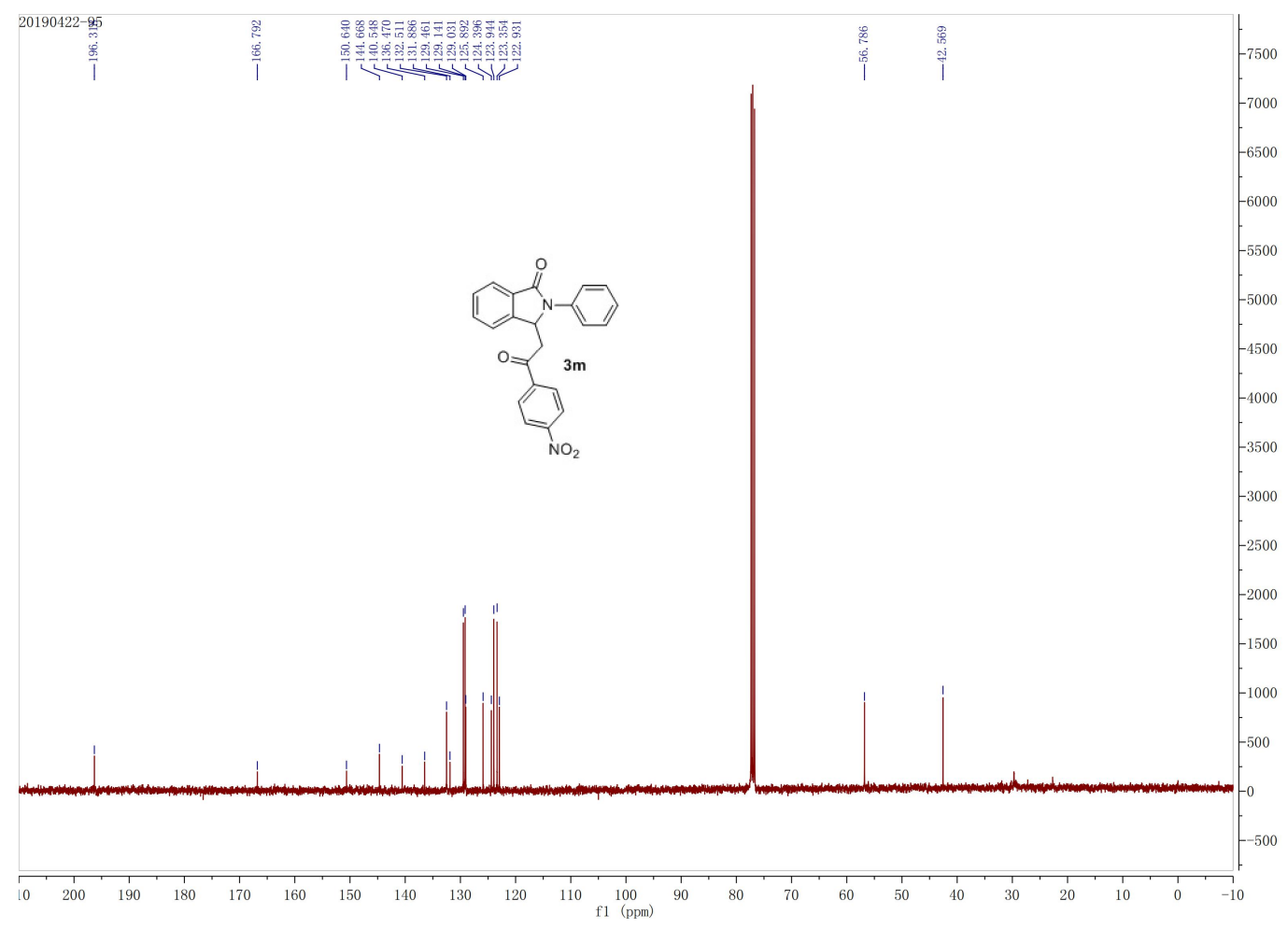

Figure S26. ${ }^{13} \mathrm{CNMR}\left(100 \mathrm{MHz}, \mathrm{CDCl}_{3}\right)$ of compound $3 \mathrm{~m}$ 


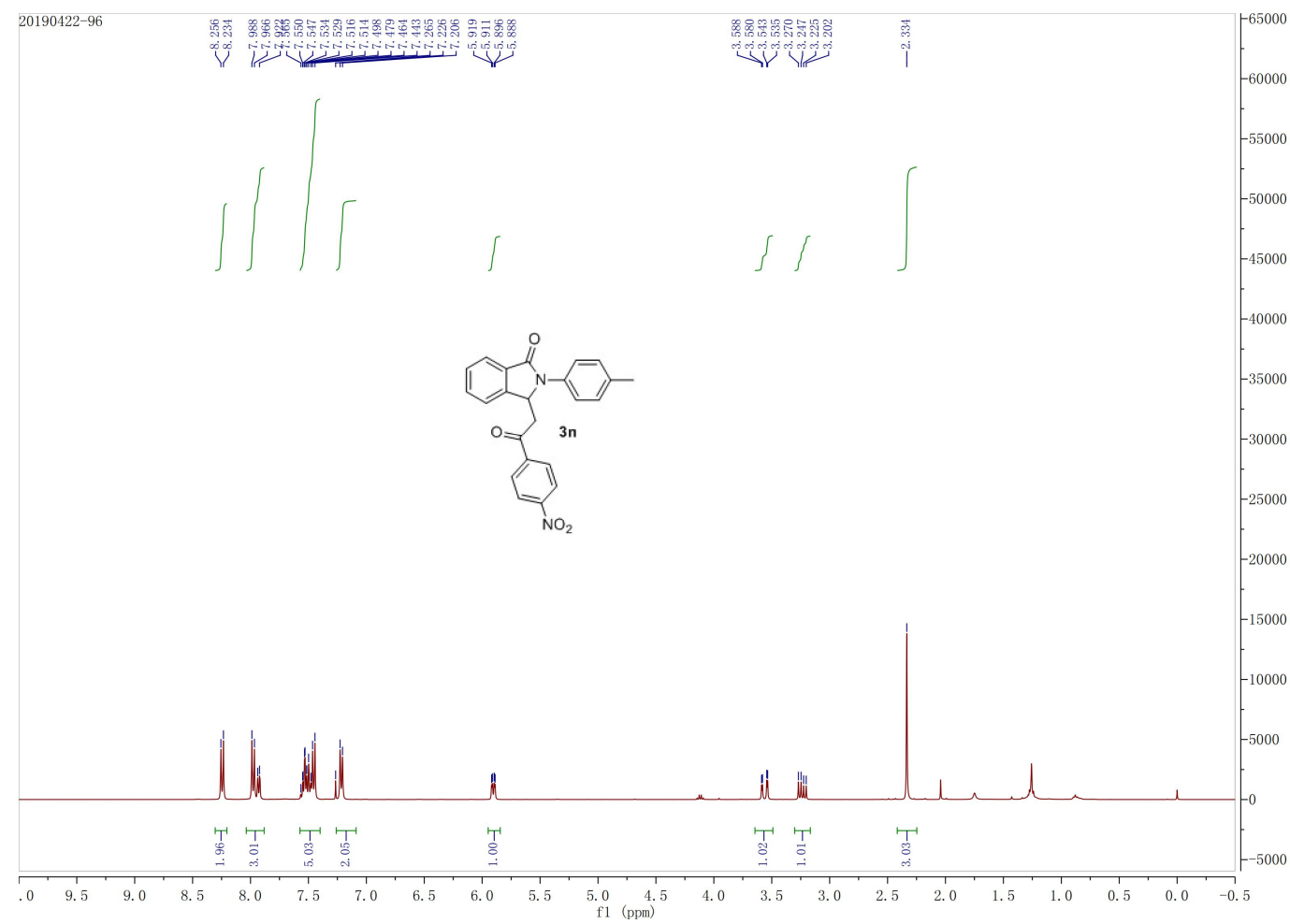

Figure S27. ${ }^{1} \mathrm{HNMR}\left(400 \mathrm{MHz}, \mathrm{CDCl}_{3}\right)$ of compound $3 \mathrm{n}$

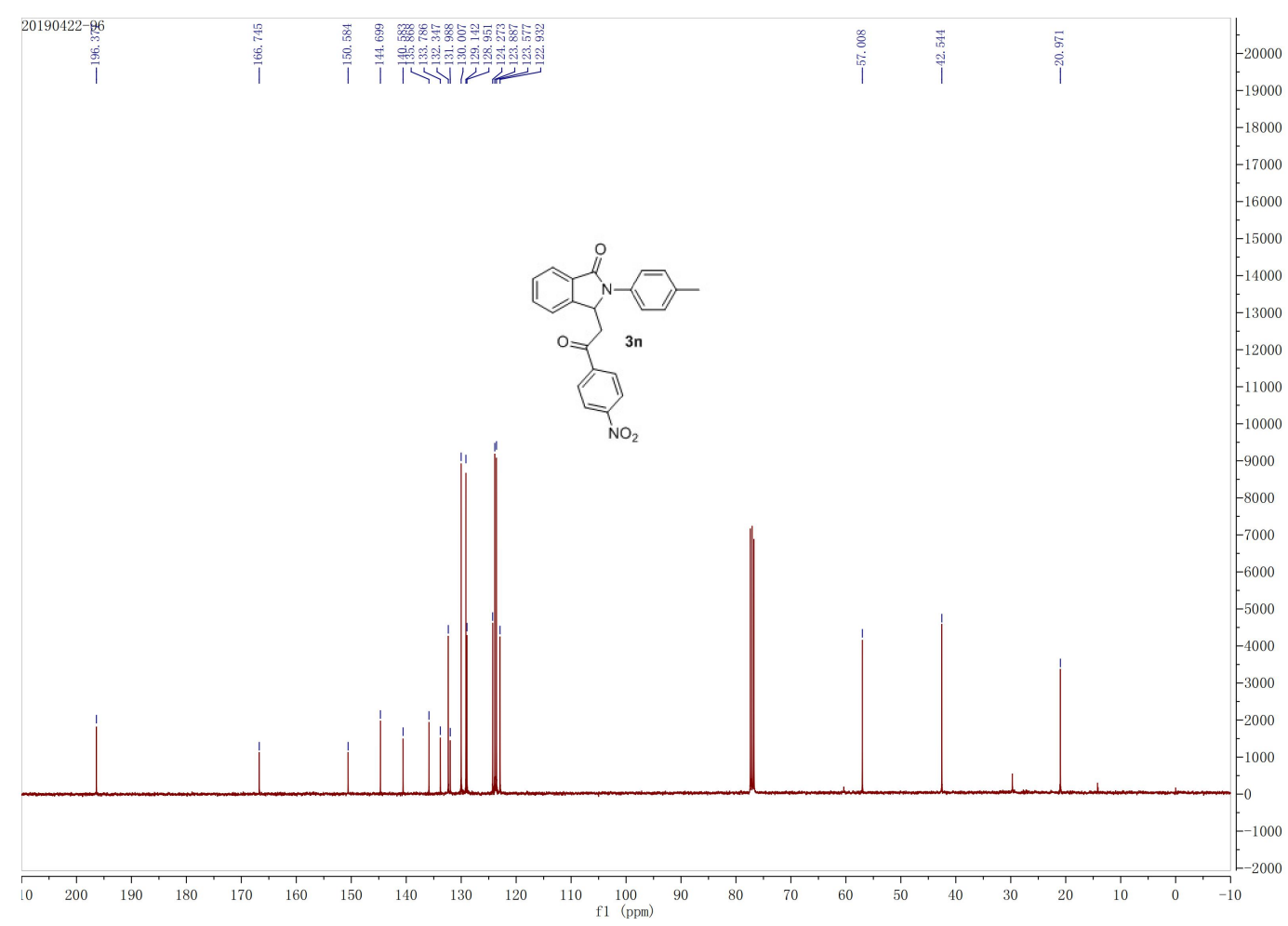

Figure S28. ${ }^{13} \mathrm{CNMR}\left(100 \mathrm{MHz}, \mathrm{CDCl}_{3}\right)$ of compound 3n 


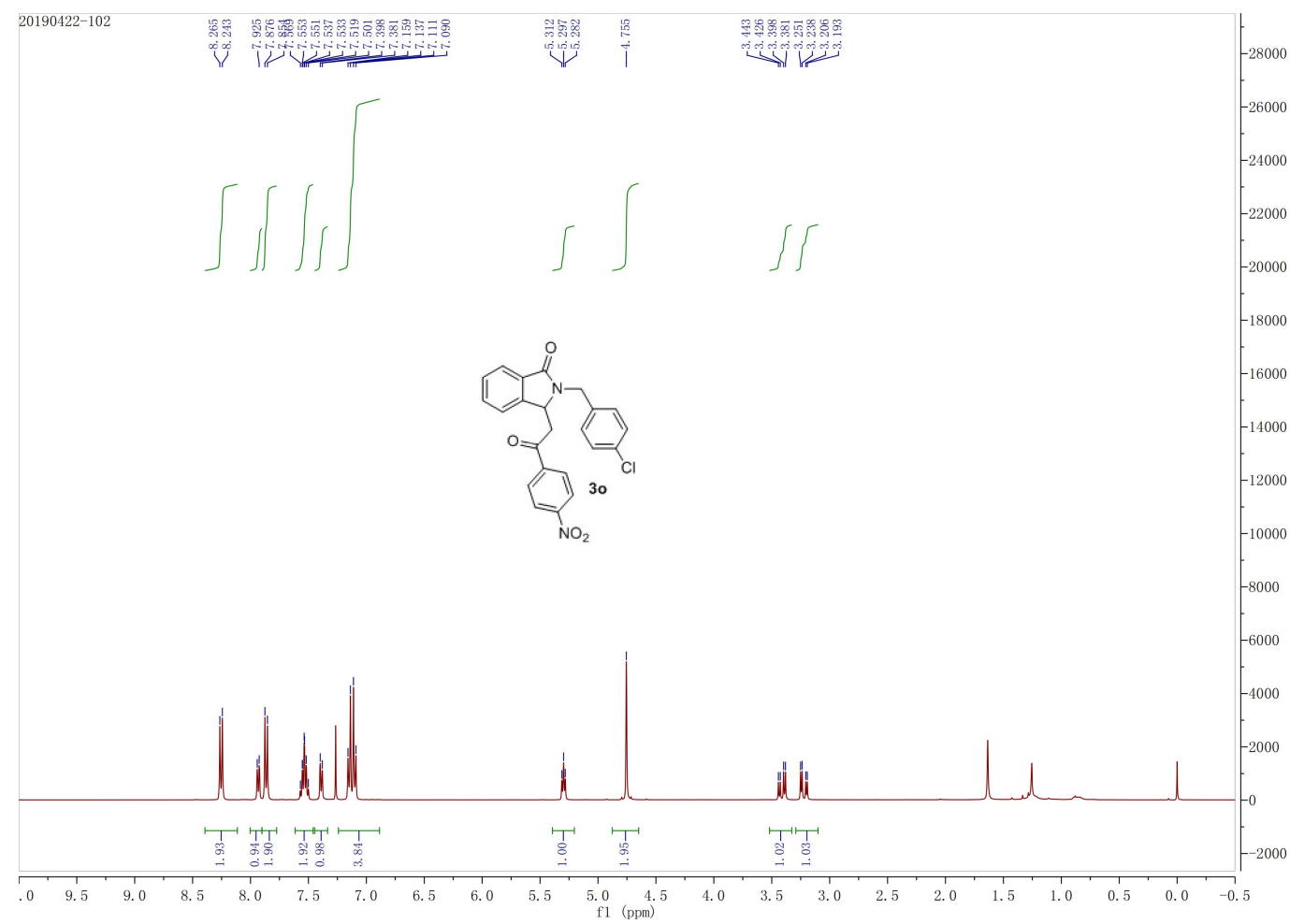

Figure S29. ${ }^{1} \mathrm{HNMR}\left(400 \mathrm{MHz}, \mathrm{CDCl}_{3}\right)$ of compound $3 \mathrm{o}$

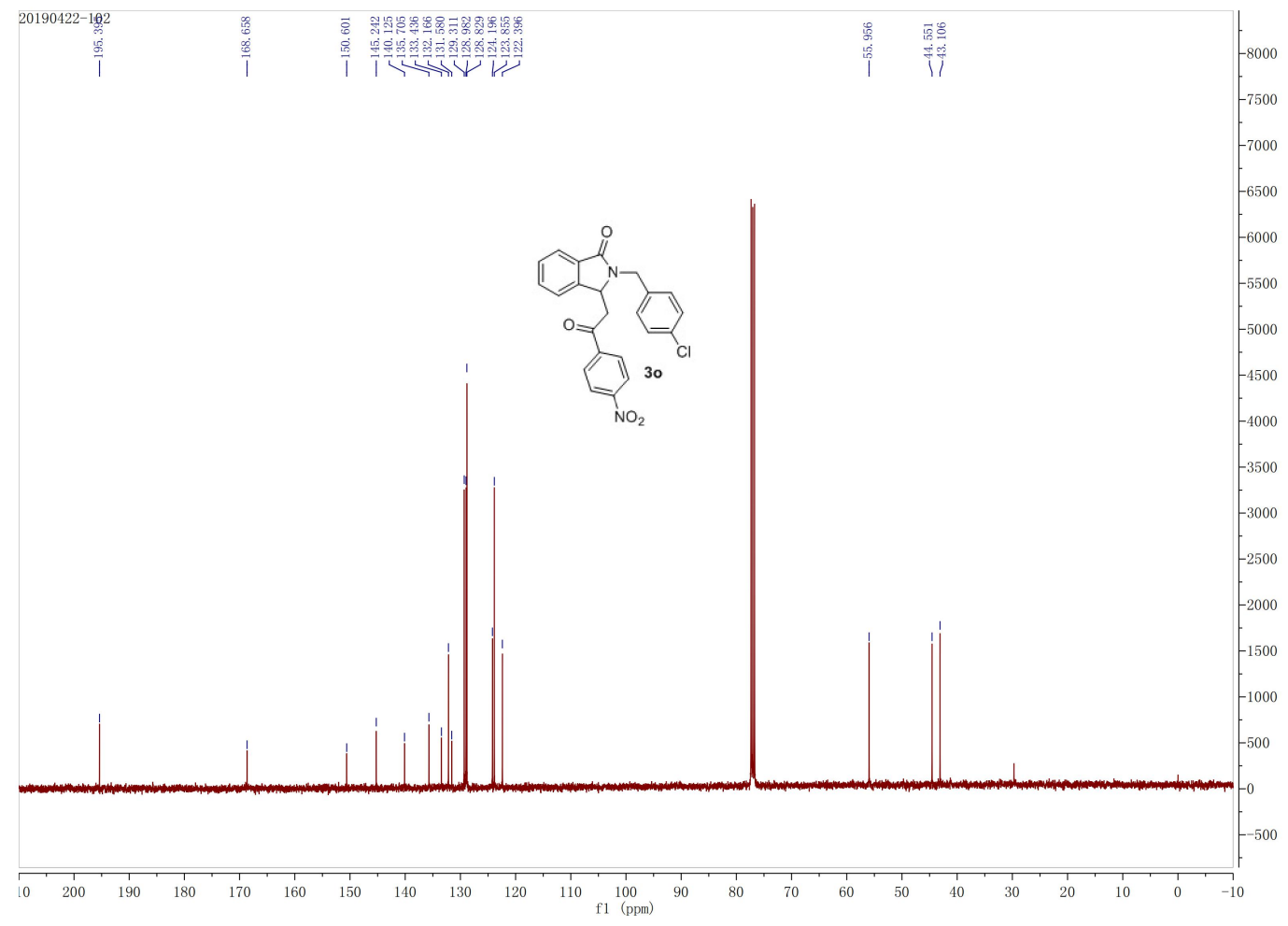

Figure S30. ${ }^{13} \mathrm{CNMR}\left(100 \mathrm{MHz}, \mathrm{CDCl}_{3}\right)$ of compound 30 


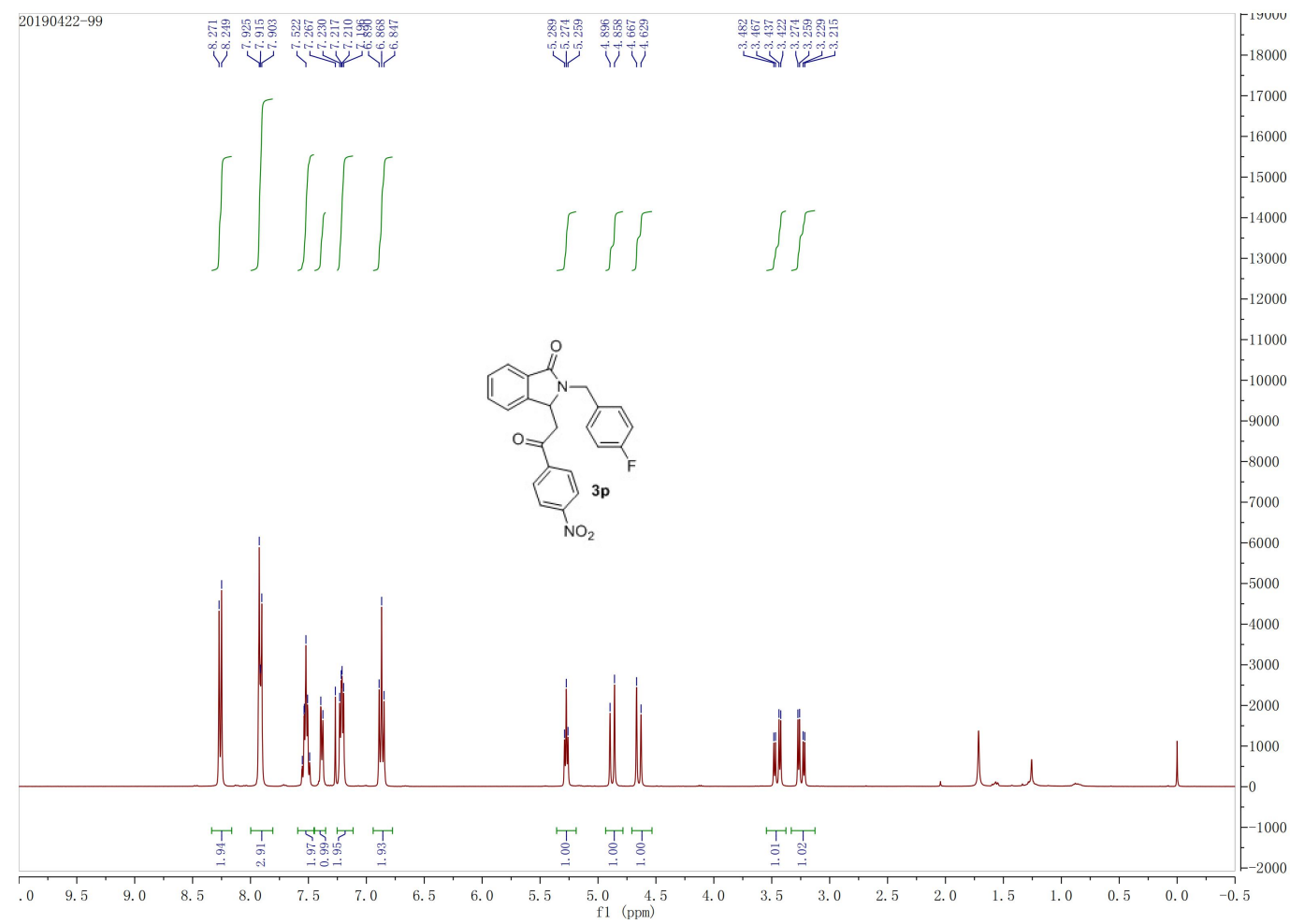

Figure S31. ${ }^{1} \mathrm{HNMR}\left(400 \mathrm{MHz}, \mathrm{CDCl}_{3}\right)$ of compound 3p

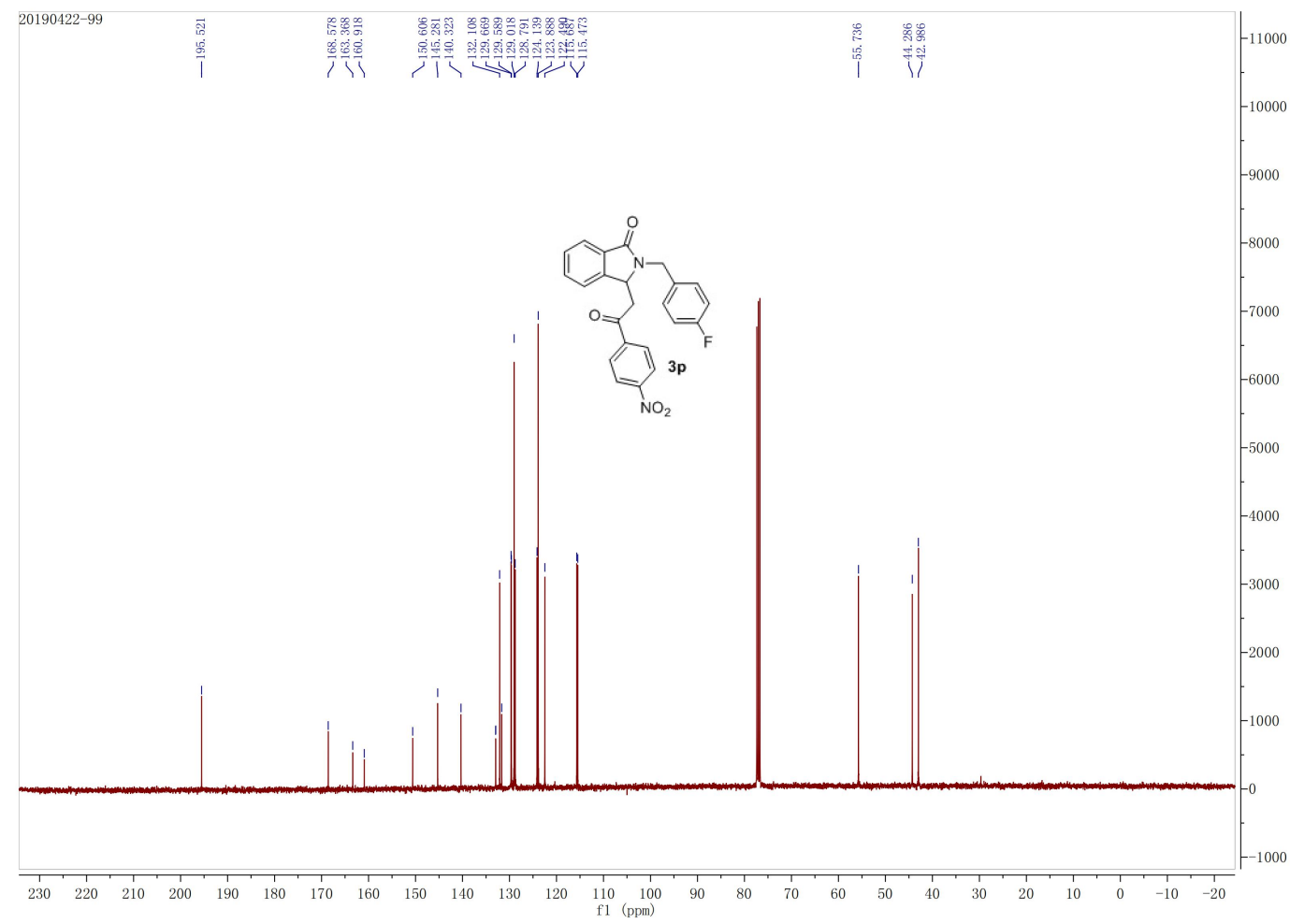

Figure S32. ${ }^{13} \mathrm{CNMR}\left(100 \mathrm{MHz}, \mathrm{CDCl}_{3}\right)$ of compound 3p 


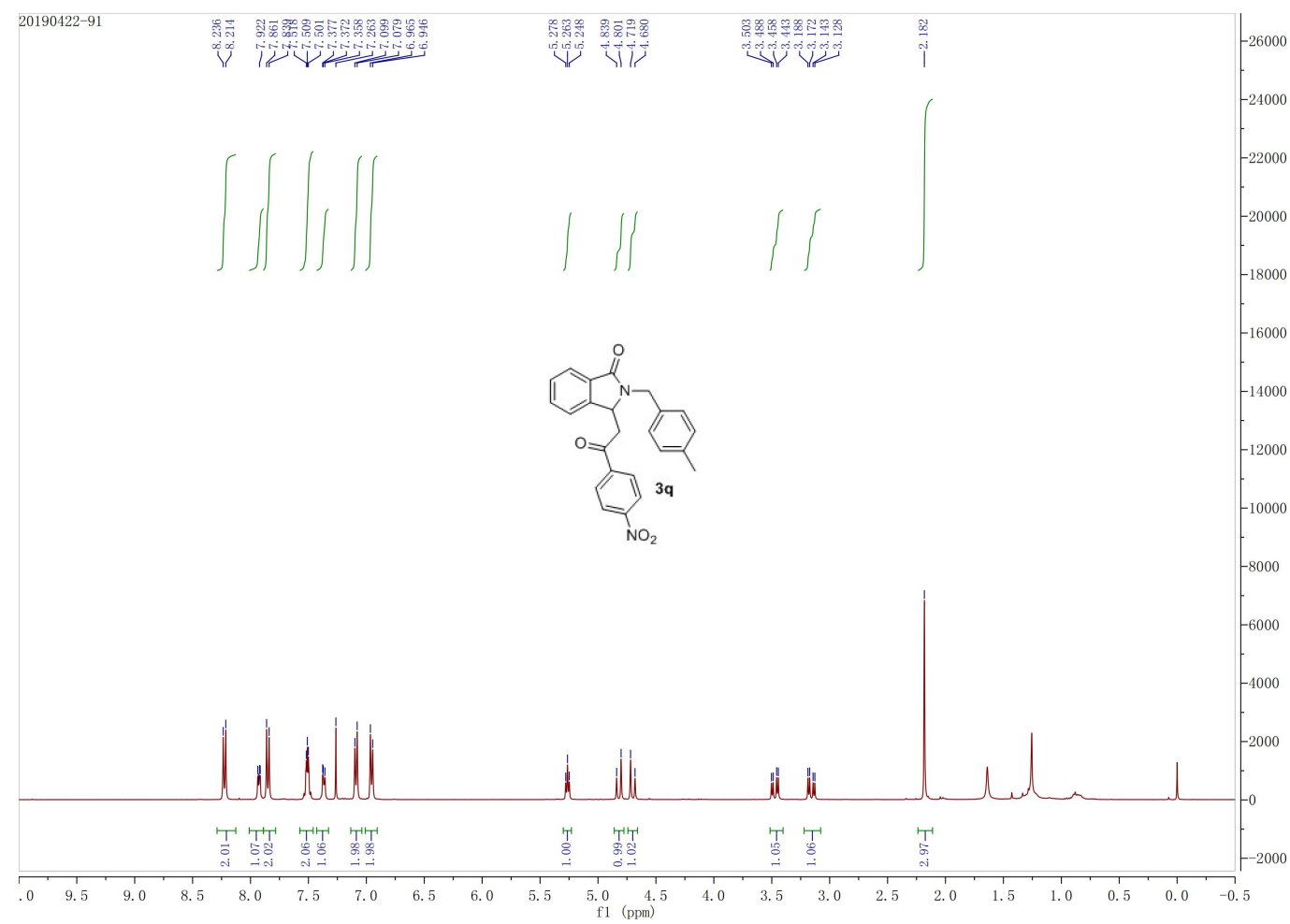

Figure S33. ${ }^{1} \mathrm{HNMR}\left(400 \mathrm{MHz}, \mathrm{CDCl}_{3}\right)$ of compound 3q

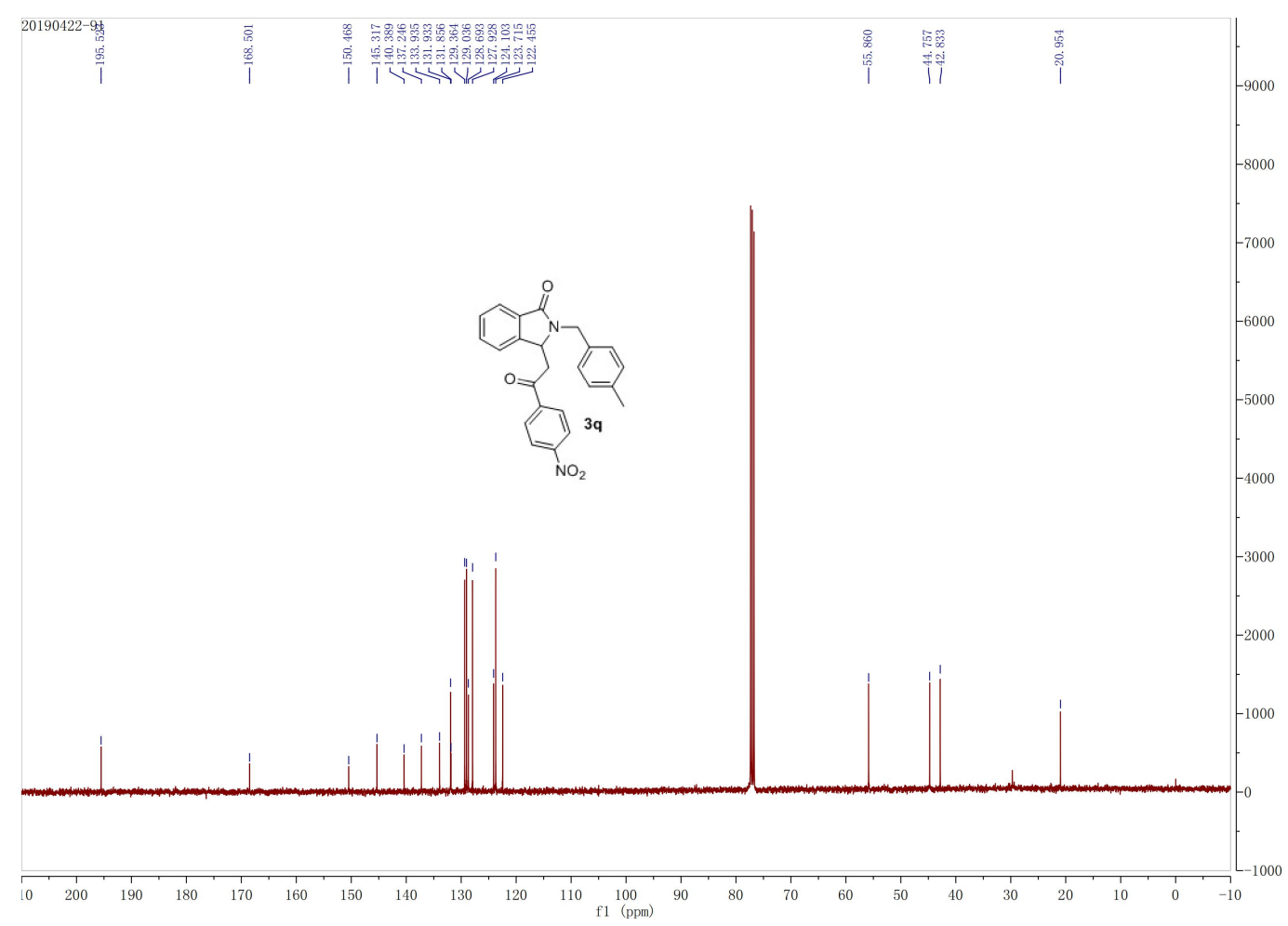

Figure S34. ${ }^{13} \mathrm{CNMR}\left(100 \mathrm{MHz}, \mathrm{CDCl}_{3}\right)$ of compound 3q 


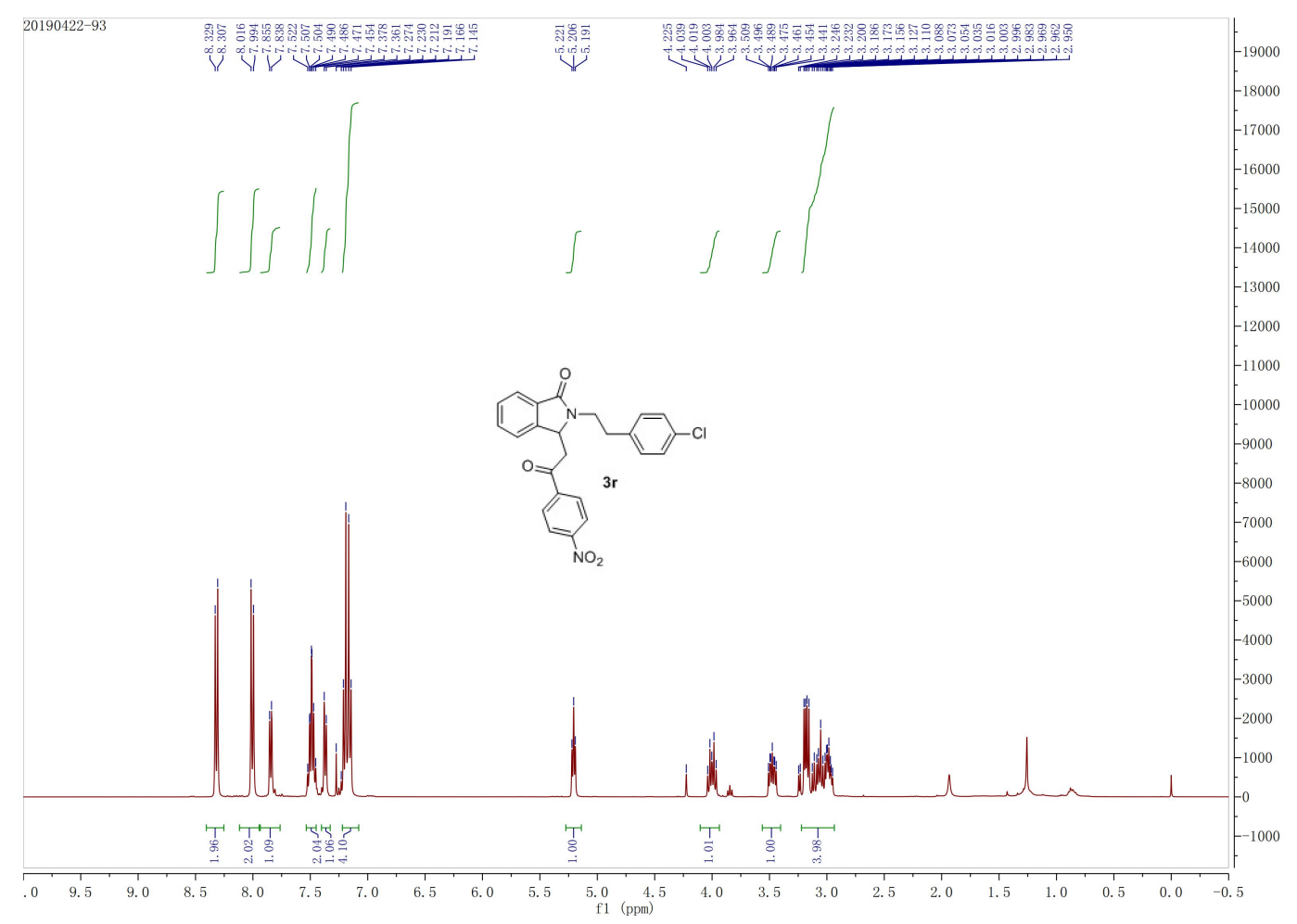

Figure S35. ${ }^{1} \mathrm{HNMR}\left(400 \mathrm{MHz}, \mathrm{CDCl}_{3}\right)$ of compound $3 \mathrm{r}$

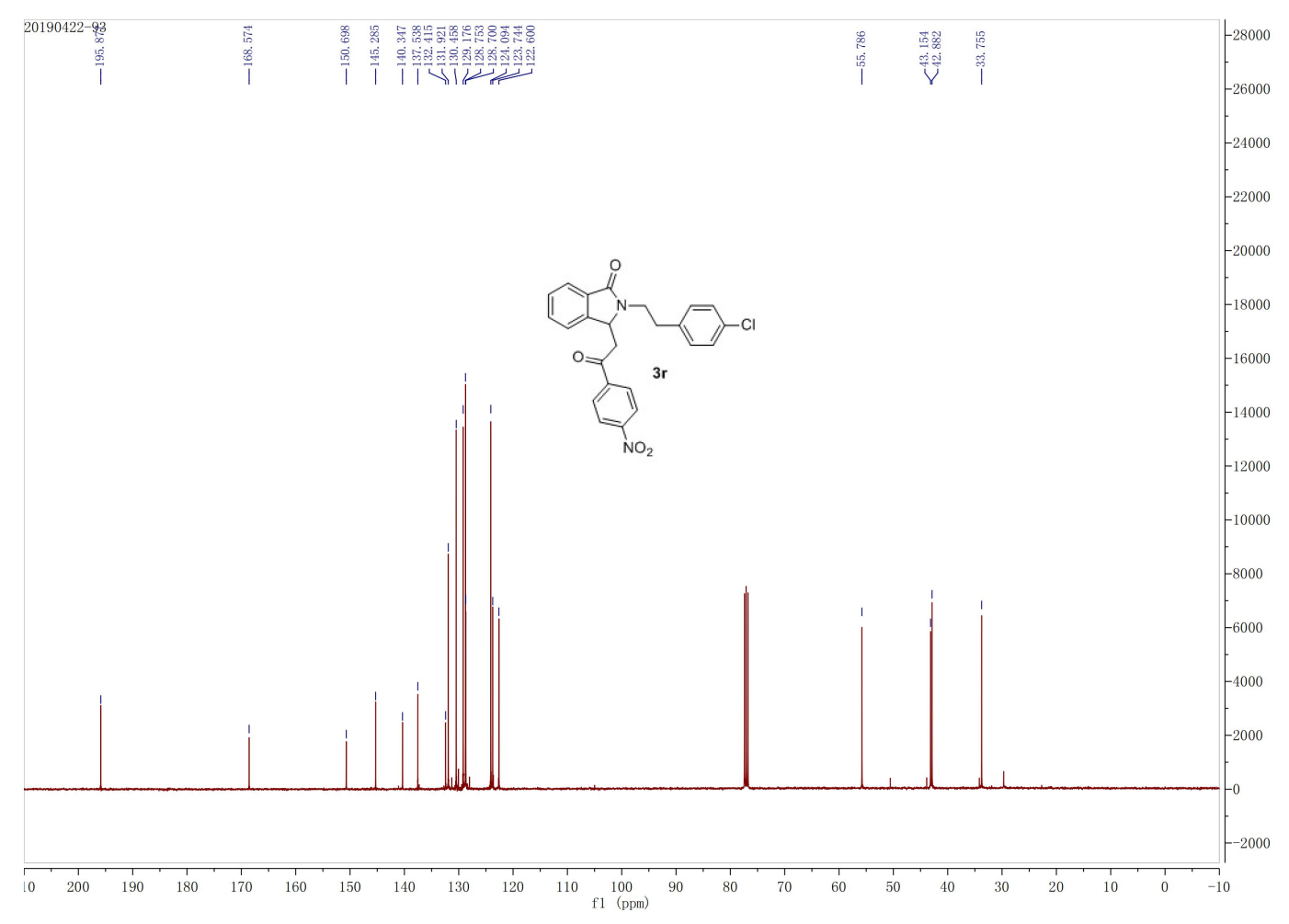

Figure S36. ${ }^{13} \mathrm{CNMR}\left(100 \mathrm{MHz}, \mathrm{CDCl}_{3}\right)$ of compound 3r 


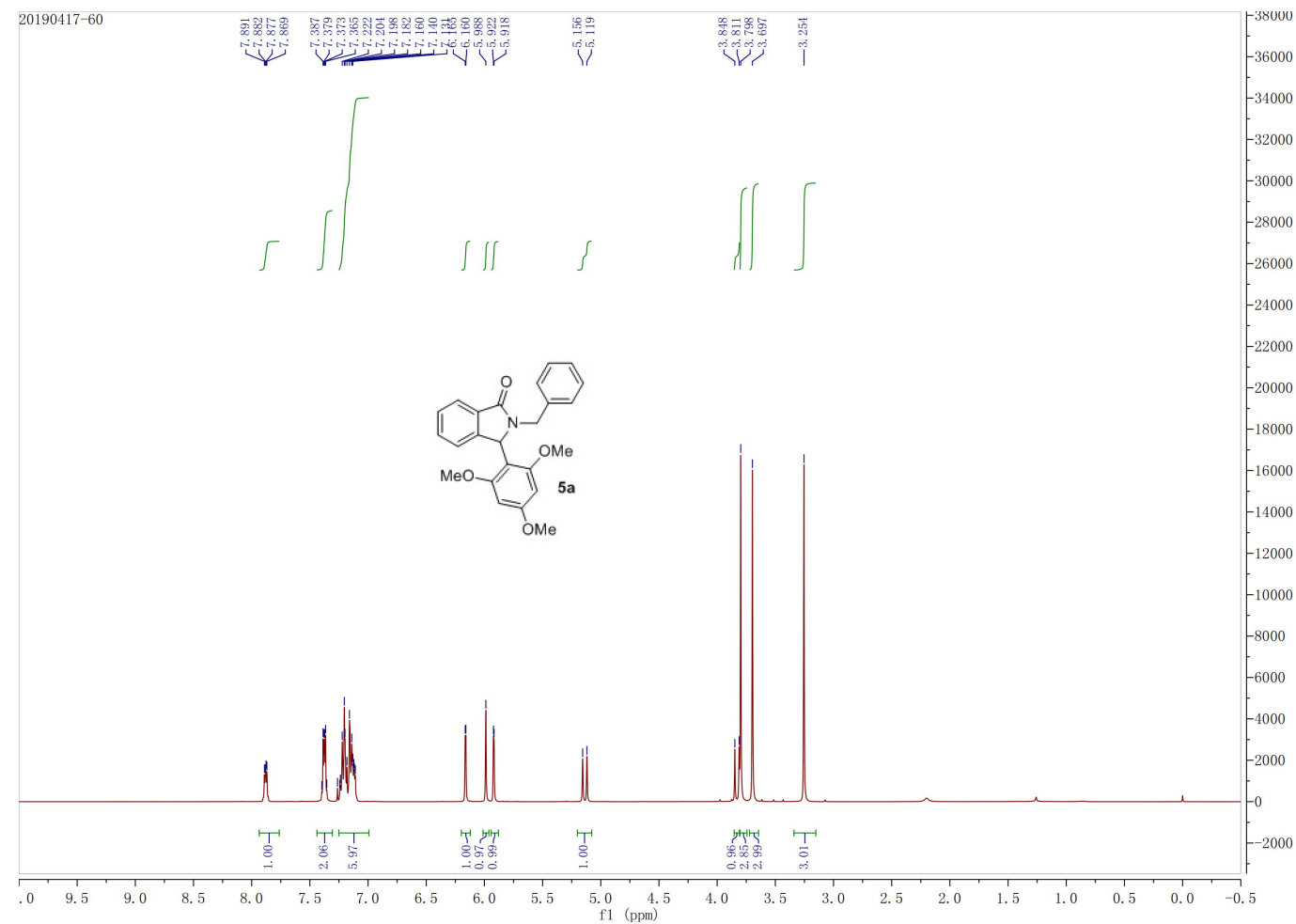

Figure S37. ${ }^{1} \mathrm{HNMR}\left(400 \mathrm{MHz}, \mathrm{CDCl}_{3}\right)$ of compound $5 \mathrm{a}$

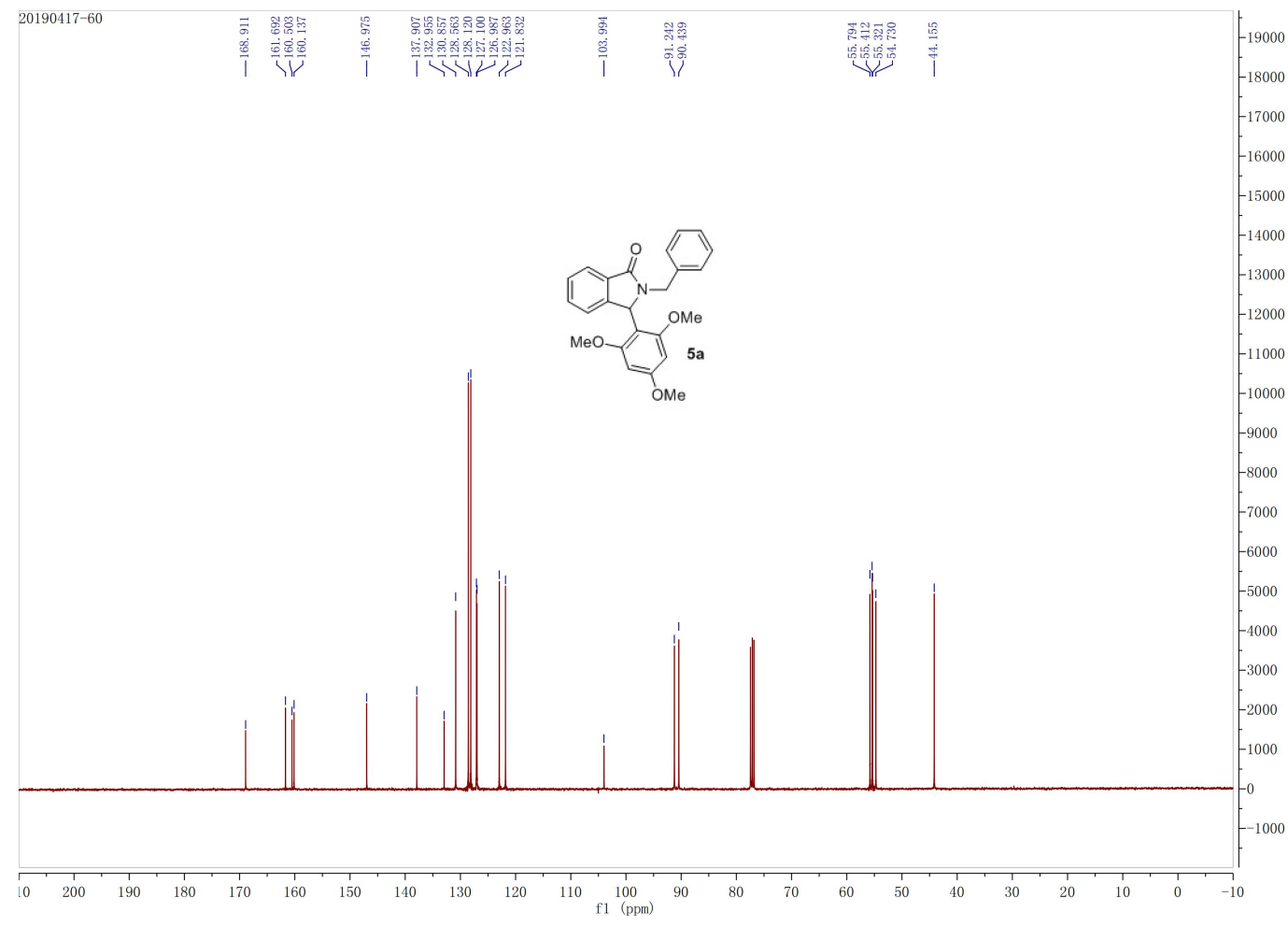

Figure S38. ${ }^{13} \mathrm{CNMR}\left(100 \mathrm{MHz}, \mathrm{CDCl}_{3}\right)$ of compound 5a 


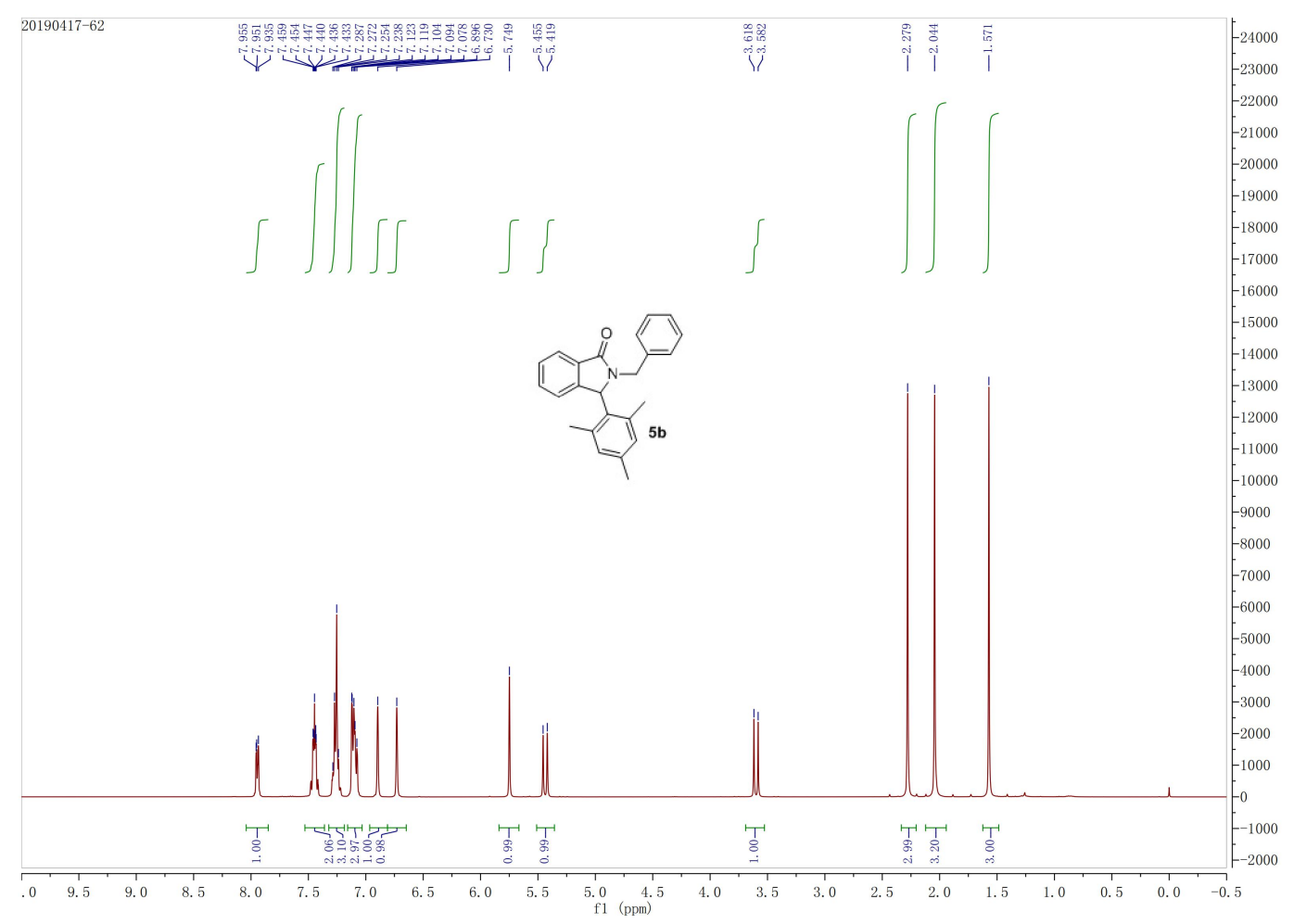

Figure S39. ${ }^{1} \mathrm{HNMR}\left(400 \mathrm{MHz}, \mathrm{CDCl}_{3}\right)$ of compound $5 \mathrm{~b}$

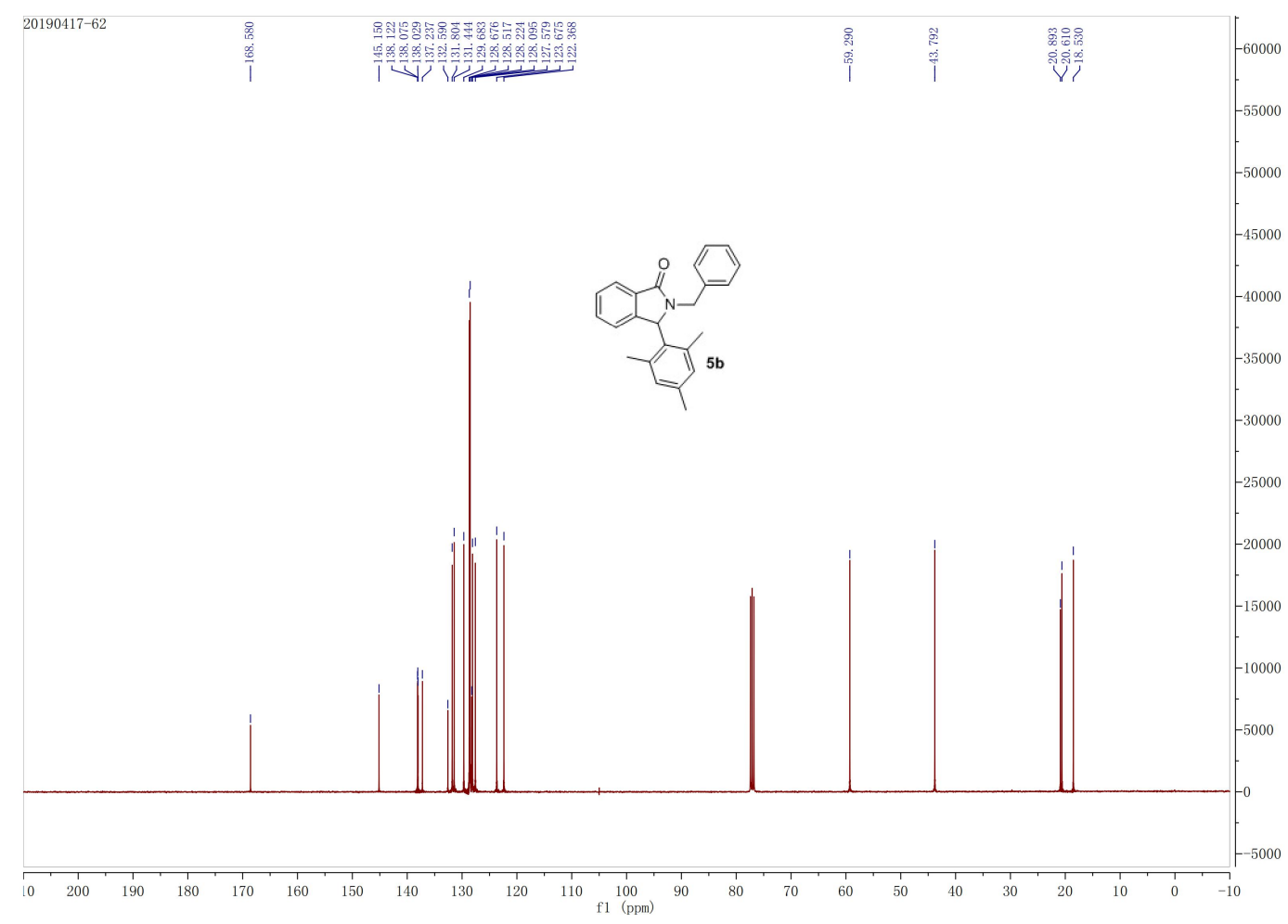

Figure S40. ${ }^{13} \mathrm{CNMR}\left(100 \mathrm{MHz}, \mathrm{CDCl}_{3}\right)$ of compound $5 \mathrm{~b}$ 


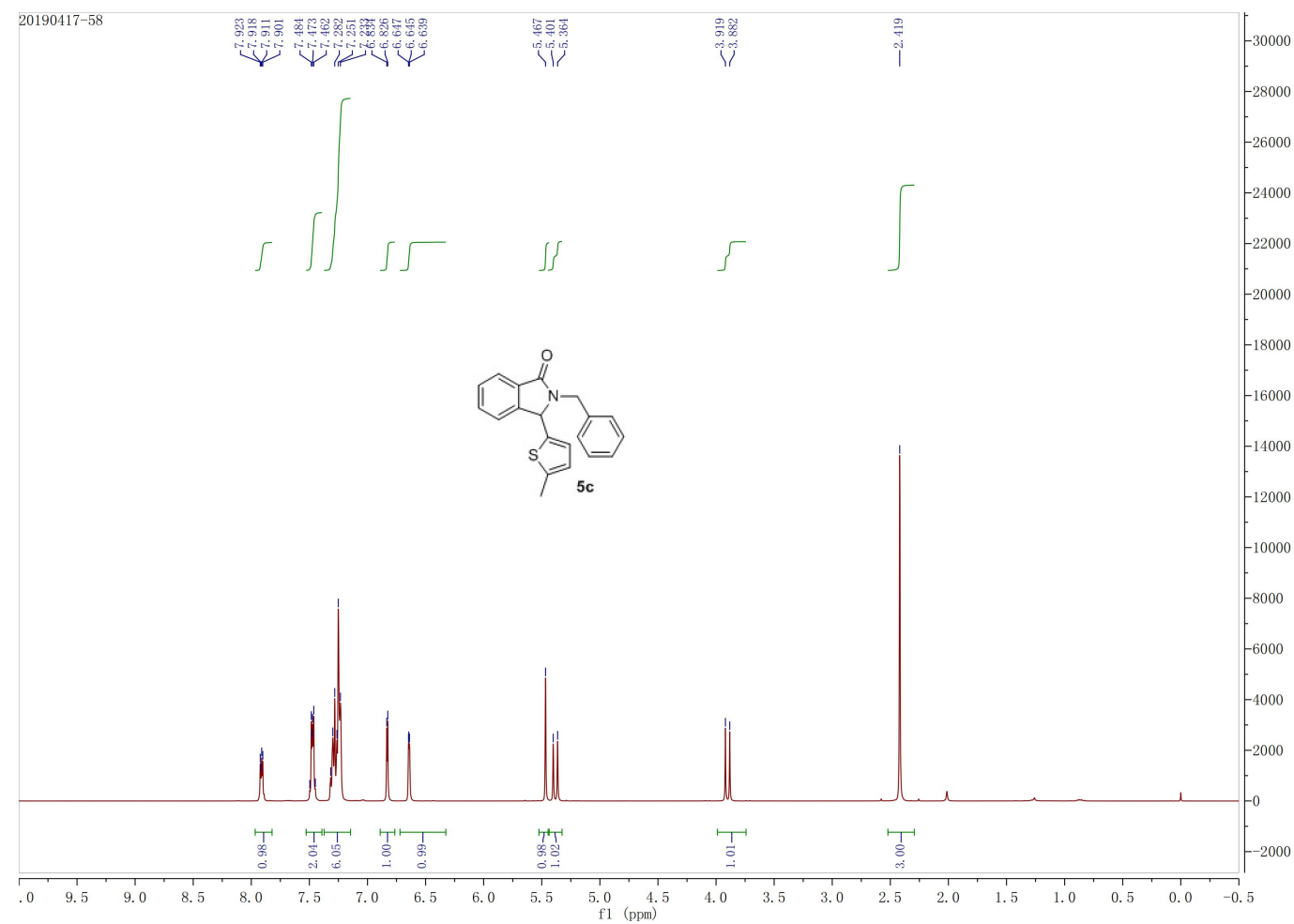

Figure S41. ${ }^{1} \mathrm{HNMR}\left(400 \mathrm{MHz}, \mathrm{CDCl}_{3}\right)$ of compound $5 \mathrm{c}$

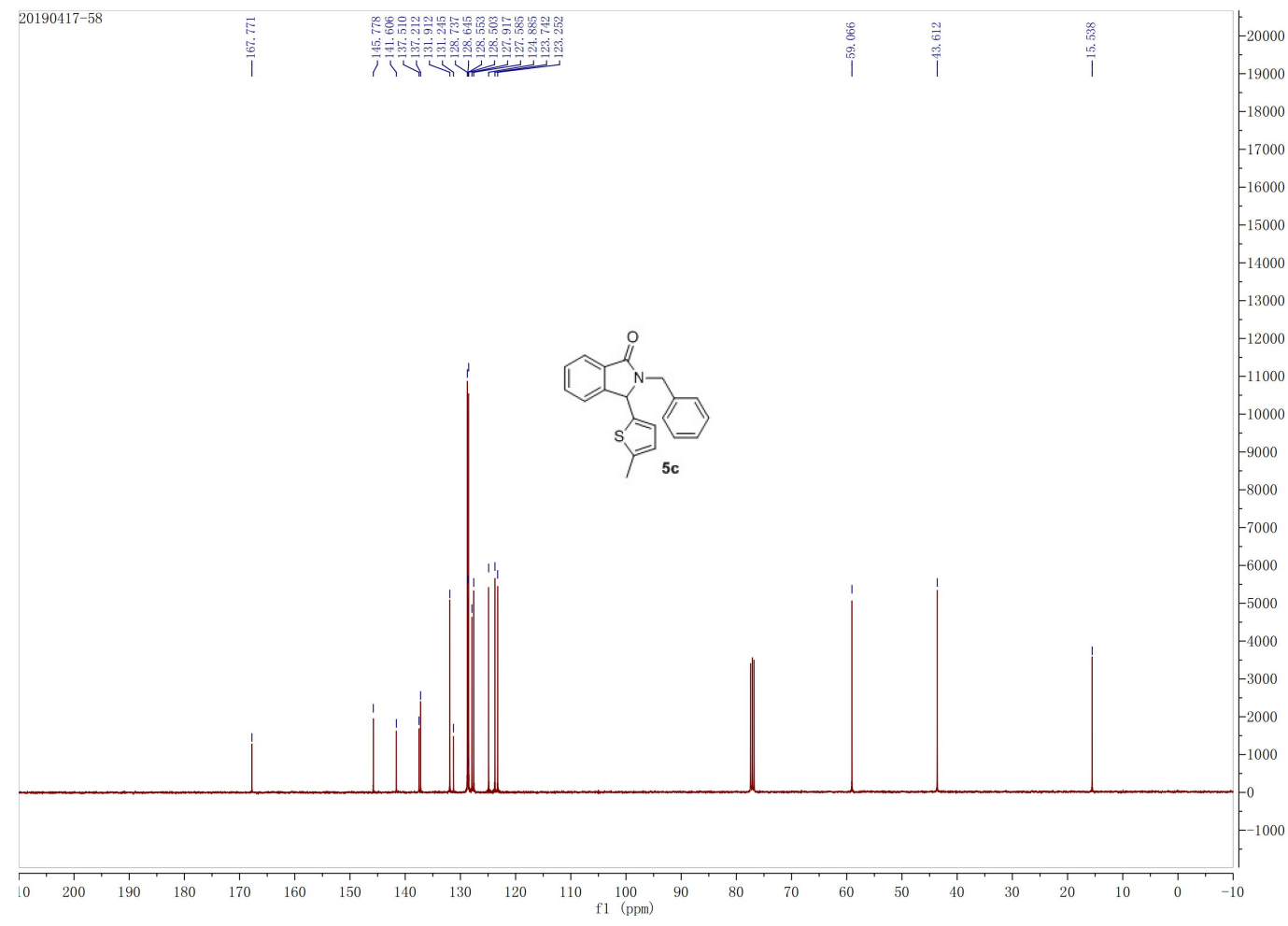

Figure S42. ${ }^{13} \mathrm{CNMR}\left(100 \mathrm{MHz}, \mathrm{CDCl}_{3}\right)$ of compound $5 \mathrm{c}$ 


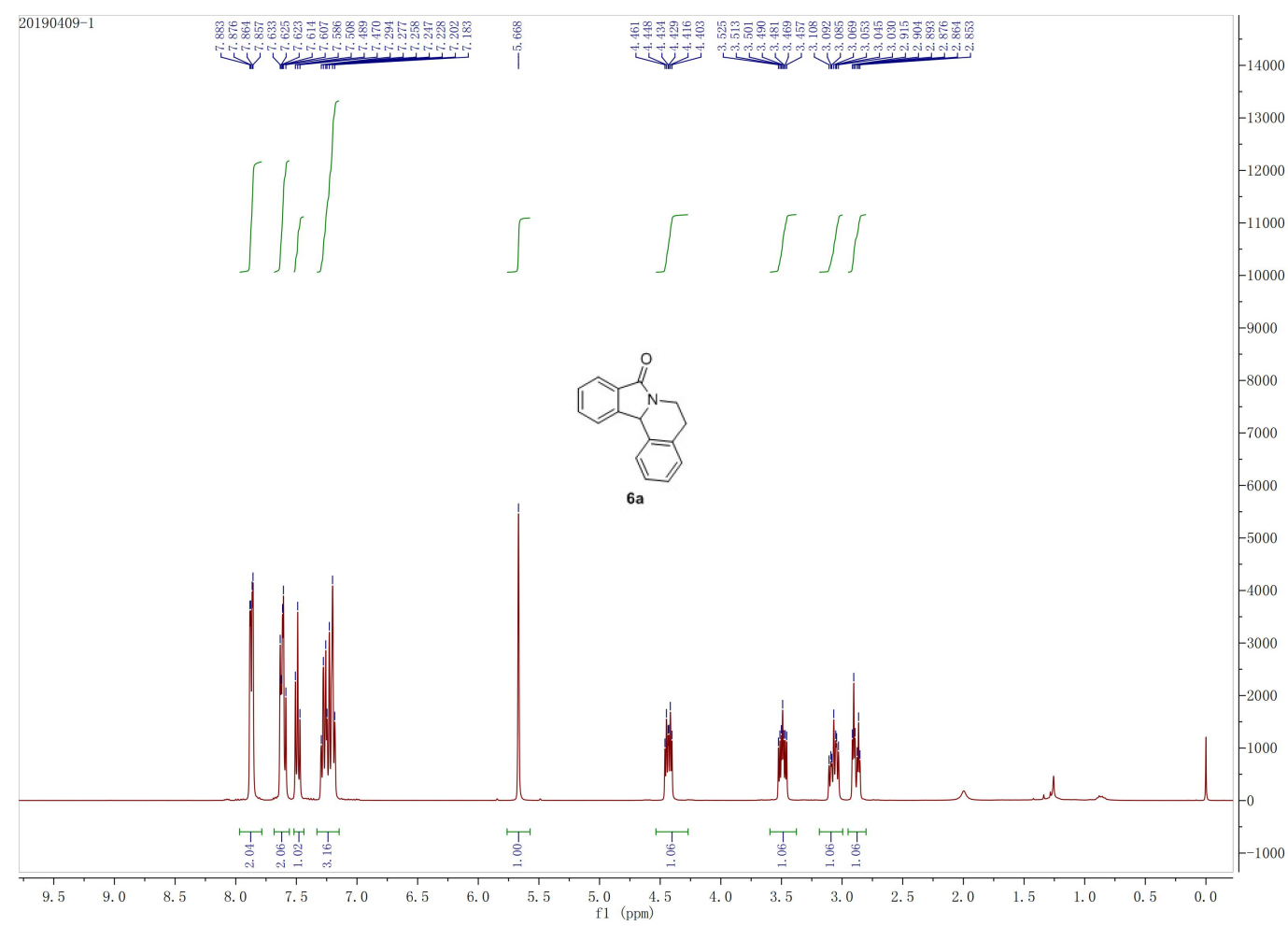

Figure S43. ${ }^{1} \mathrm{HNMR}\left(400 \mathrm{MHz}, \mathrm{CDCl}_{3}\right)$ of compound $6 \mathrm{a}$

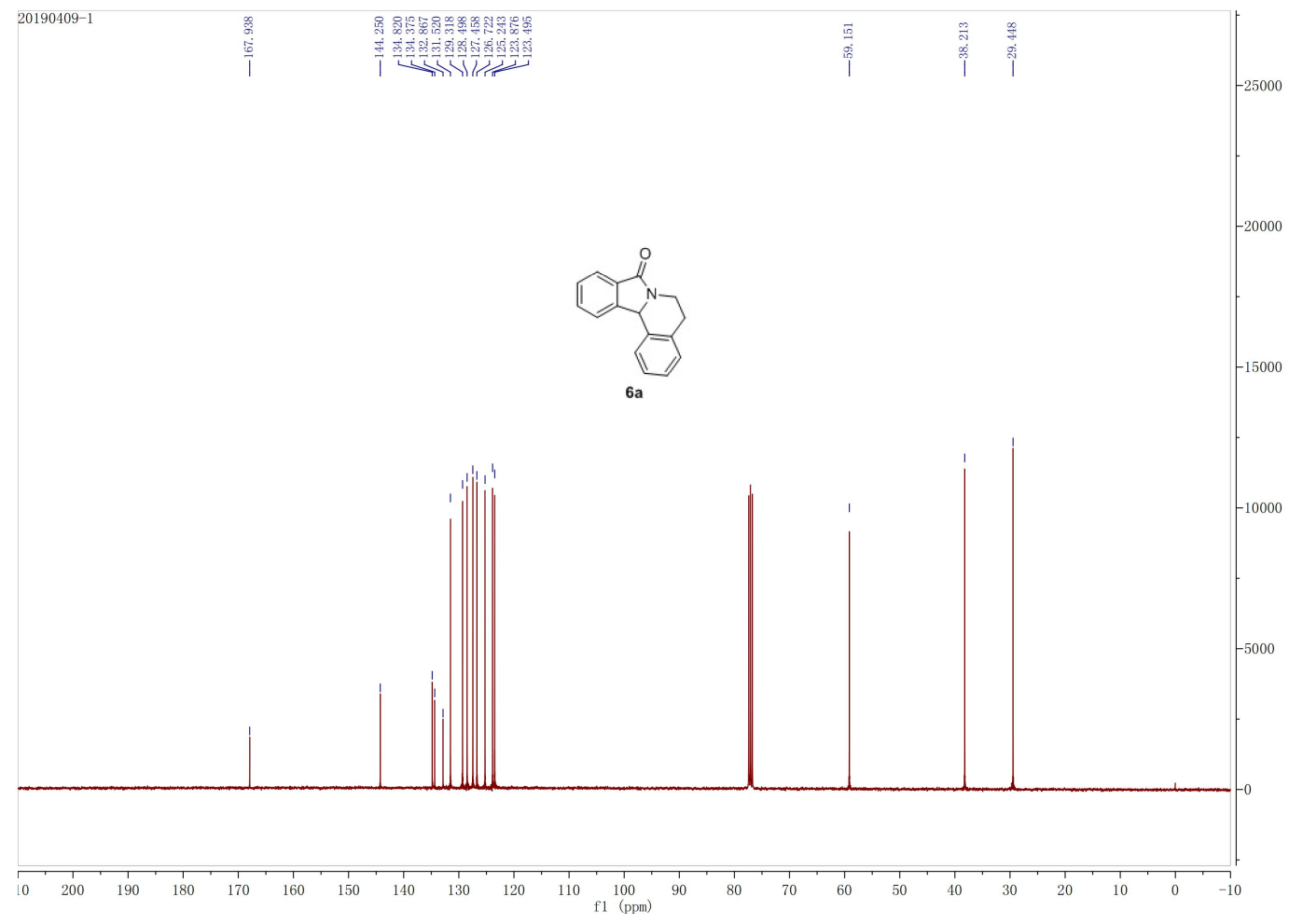

Figure S44. ${ }^{13} \mathrm{CNMR}\left(100 \mathrm{MHz}, \mathrm{CDCl}_{3}\right)$ of compound 6a 


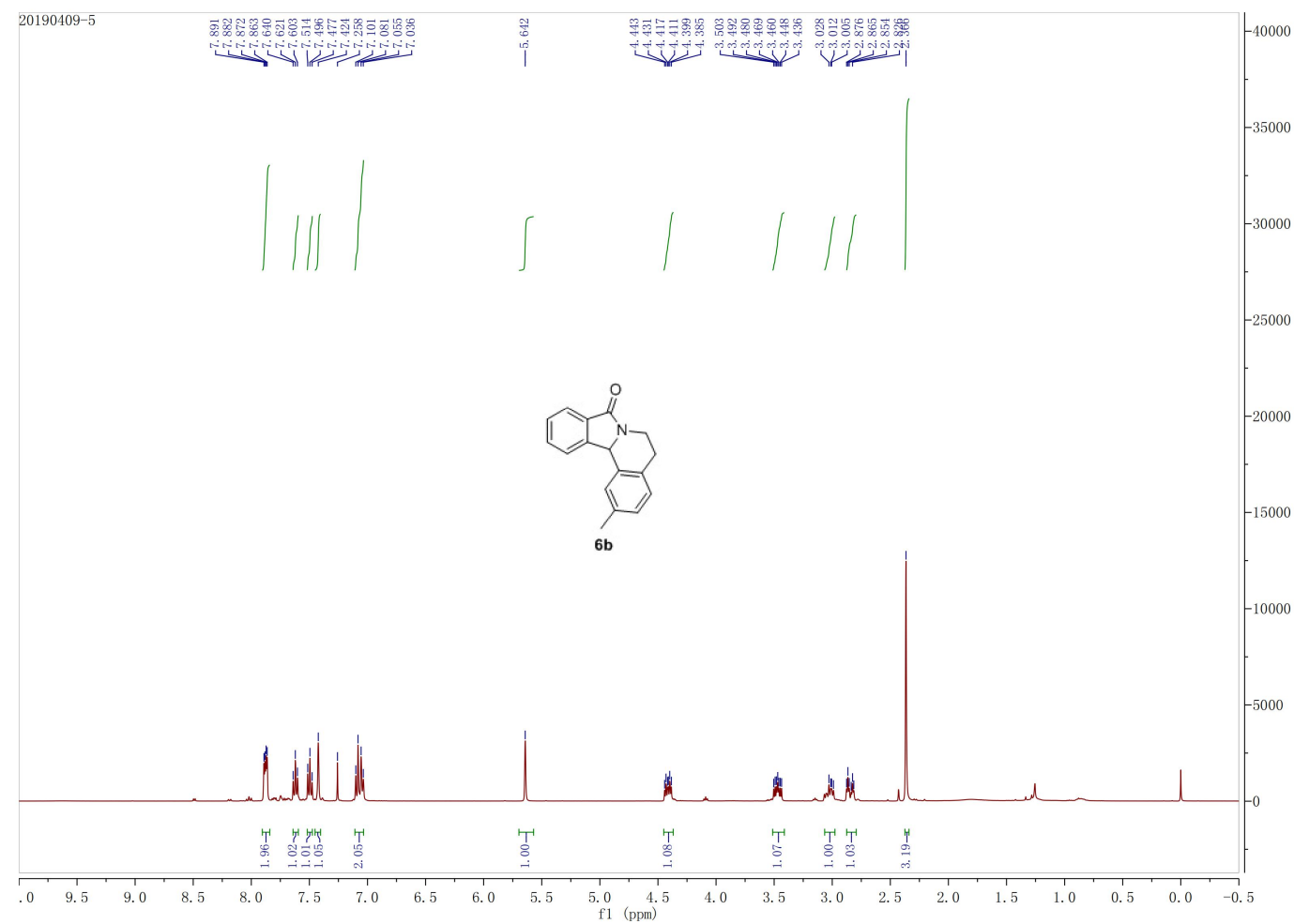

Figure S45. ${ }^{1} \mathrm{HNMR}\left(400 \mathrm{MHz}, \mathrm{CDCl}_{3}\right)$ of compound $6 \mathrm{~b}$

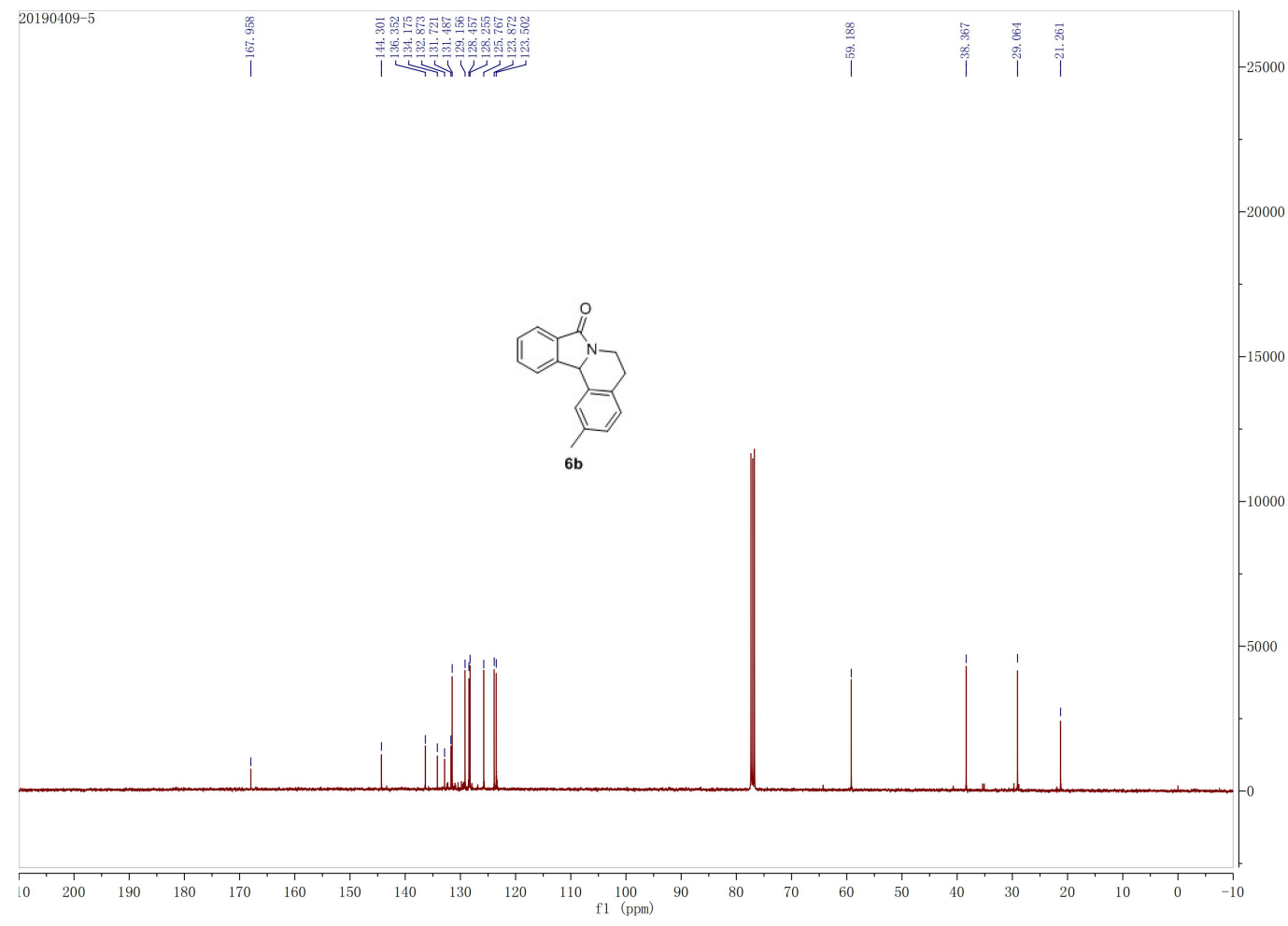

Figure S46. ${ }^{13} \mathrm{CNMR}\left(100 \mathrm{MHz}, \mathrm{CDCl}_{3}\right)$ of compound $6 \mathrm{~b}$ 


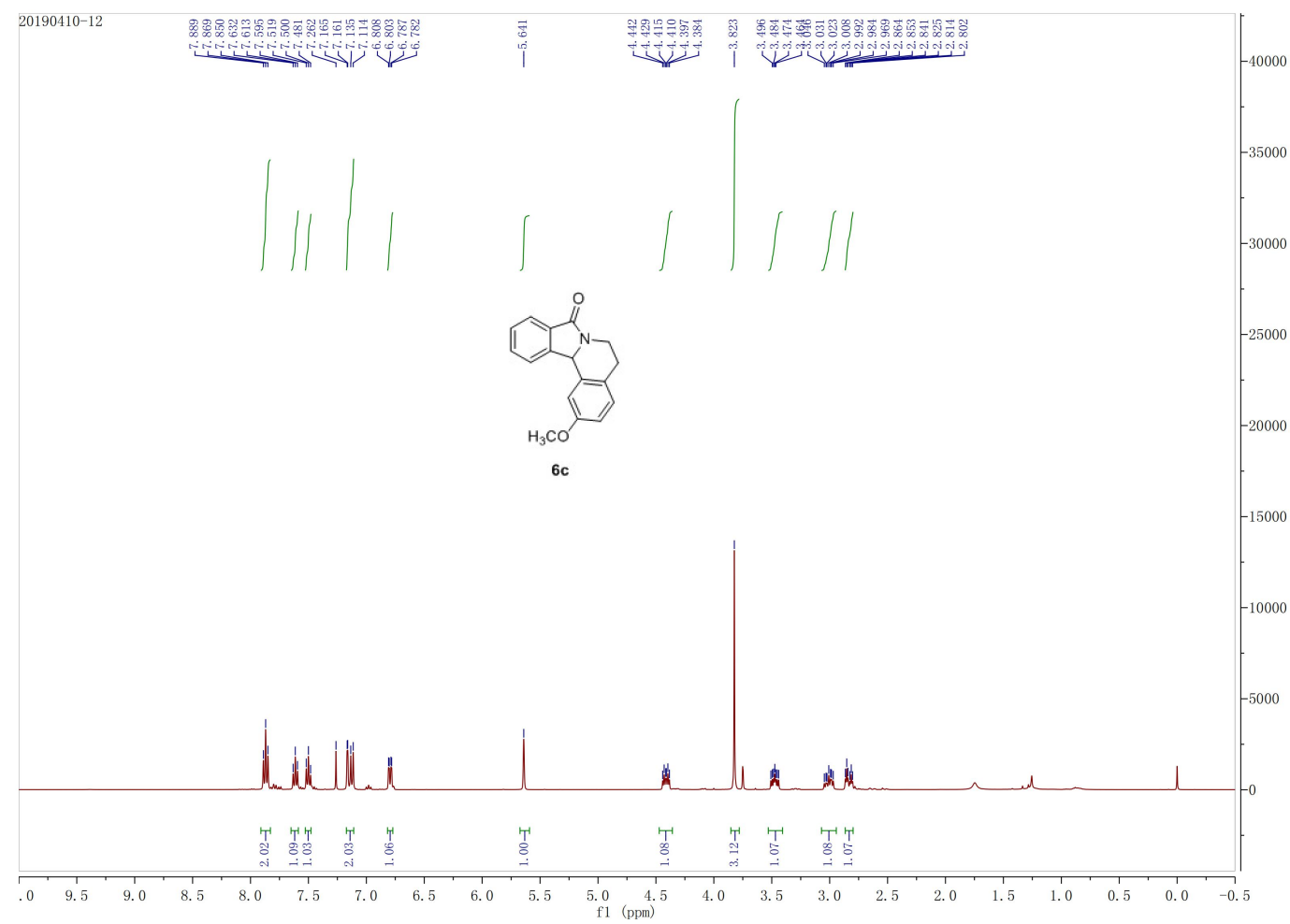

Figure S47. ${ }^{1} \mathrm{HNMR}\left(400 \mathrm{MHz}, \mathrm{CDCl}_{3}\right)$ of compound $6 \mathrm{c}$

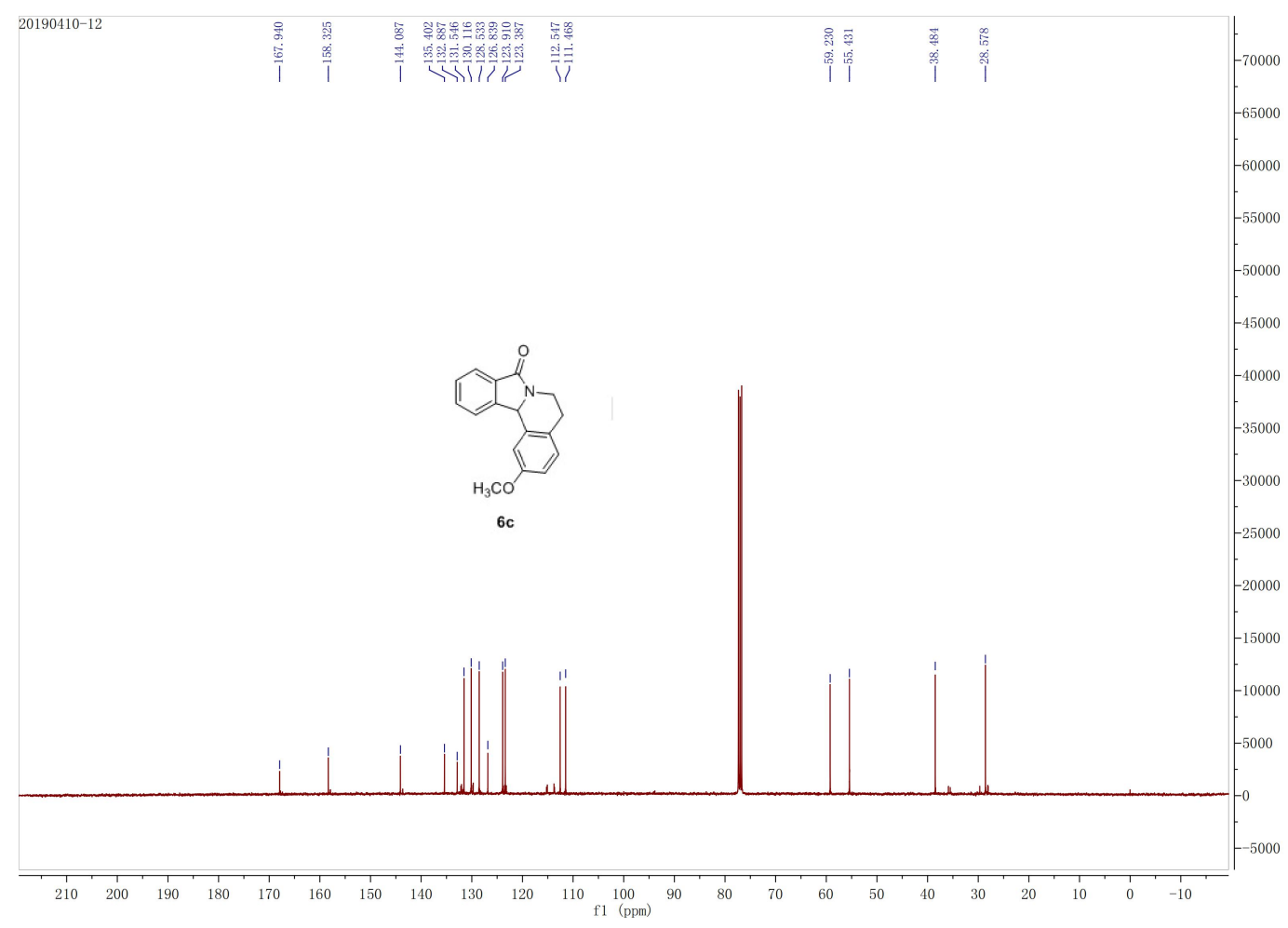

Figure S48. ${ }^{13} \mathrm{CNMR}\left(100 \mathrm{MHz}, \mathrm{CDCl}_{3}\right)$ of compound $6 \mathrm{c}$ 


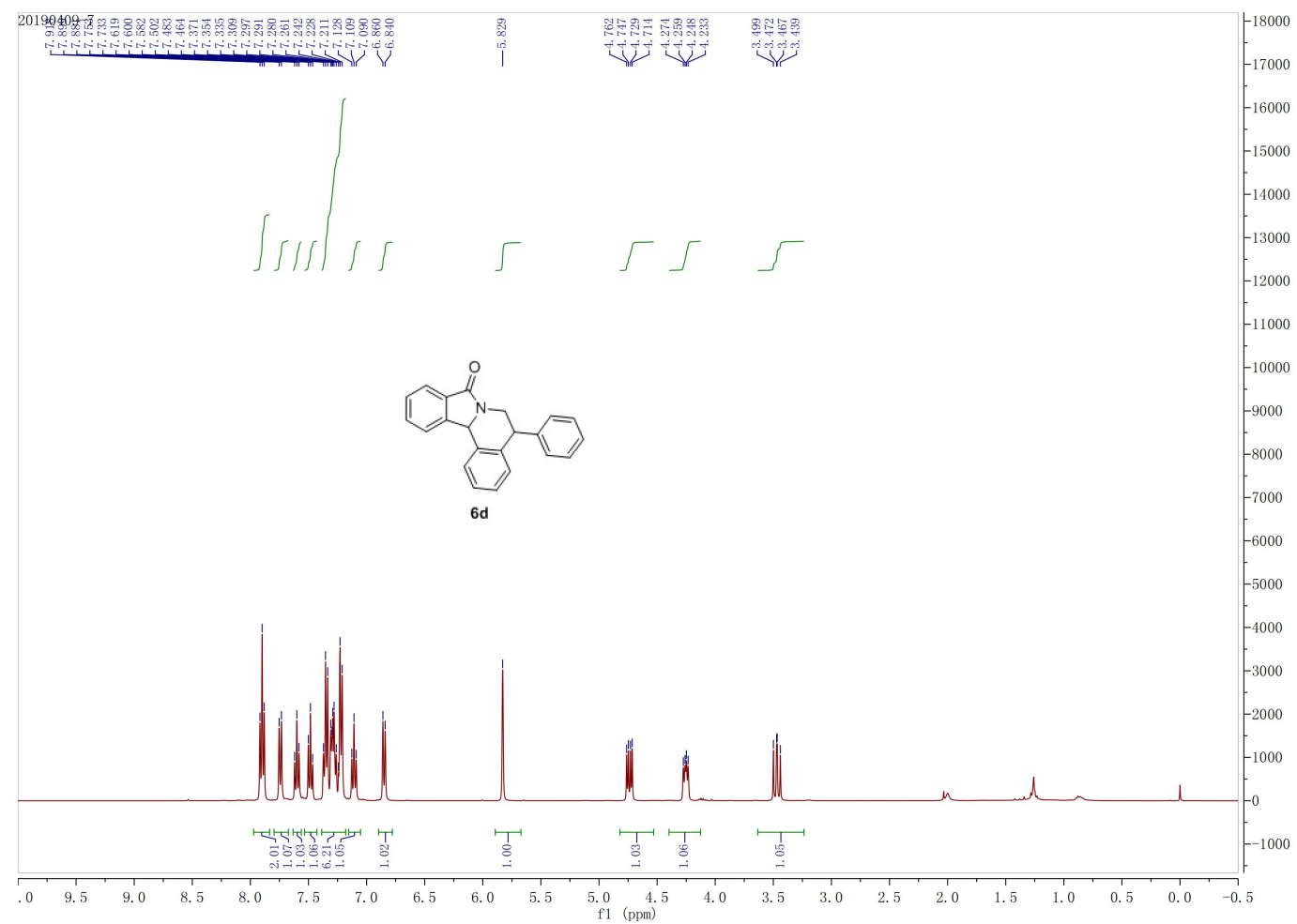

Figure S49. ${ }^{1} \mathrm{HNMR}\left(400 \mathrm{MHz}, \mathrm{CDCl}_{3}\right)$ of compound $6 \mathrm{~d}$

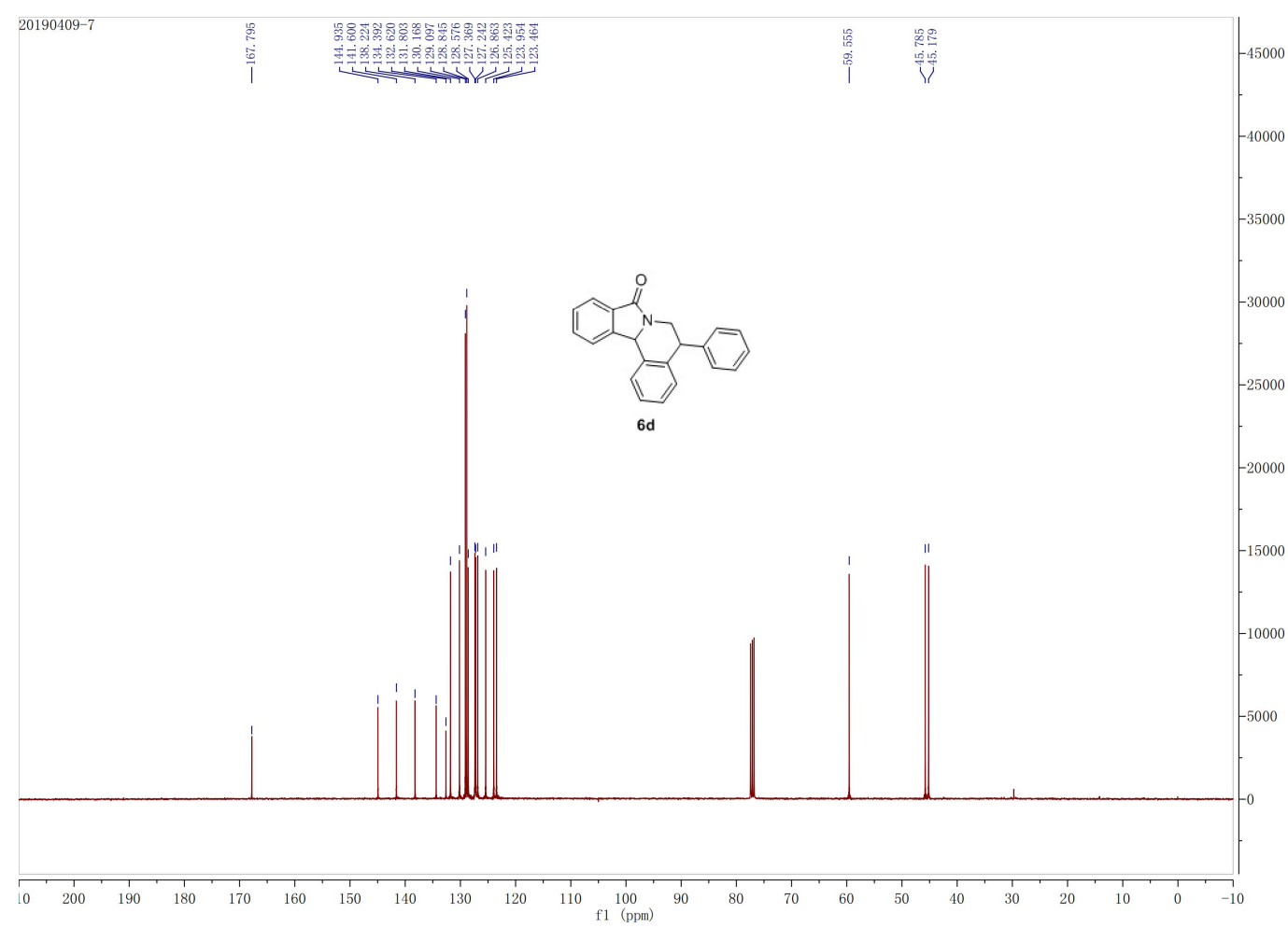

Figure S50. ${ }^{13} \mathrm{CNMR}\left(100 \mathrm{MHz}, \mathrm{CDCl}_{3}\right)$ of compound $6 \mathrm{~d}$ 


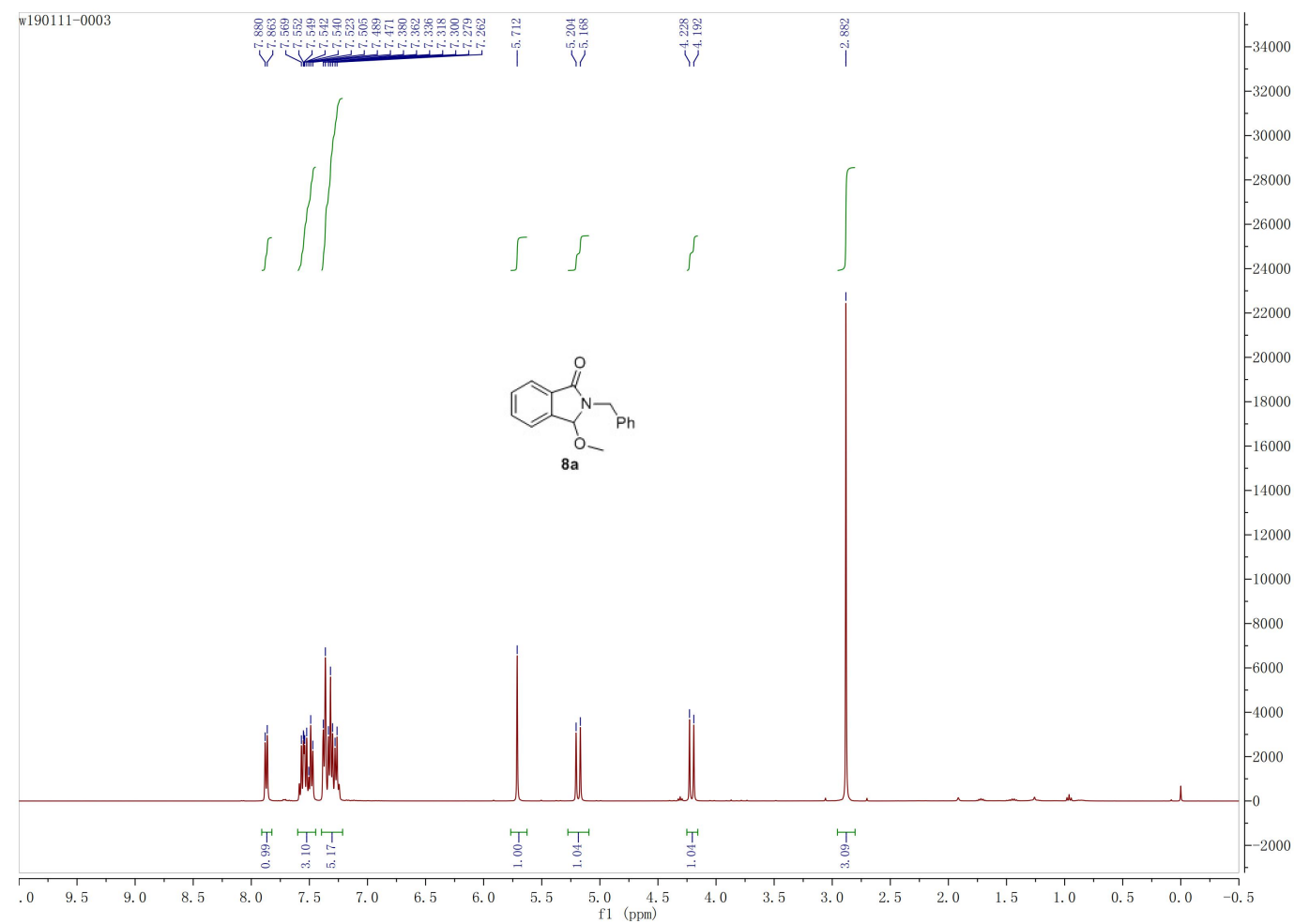

Figure S51. ${ }^{1} \mathrm{HNMR}\left(400 \mathrm{MHz}, \mathrm{CDCl}_{3}\right)$ of compound $8 \mathrm{a}$

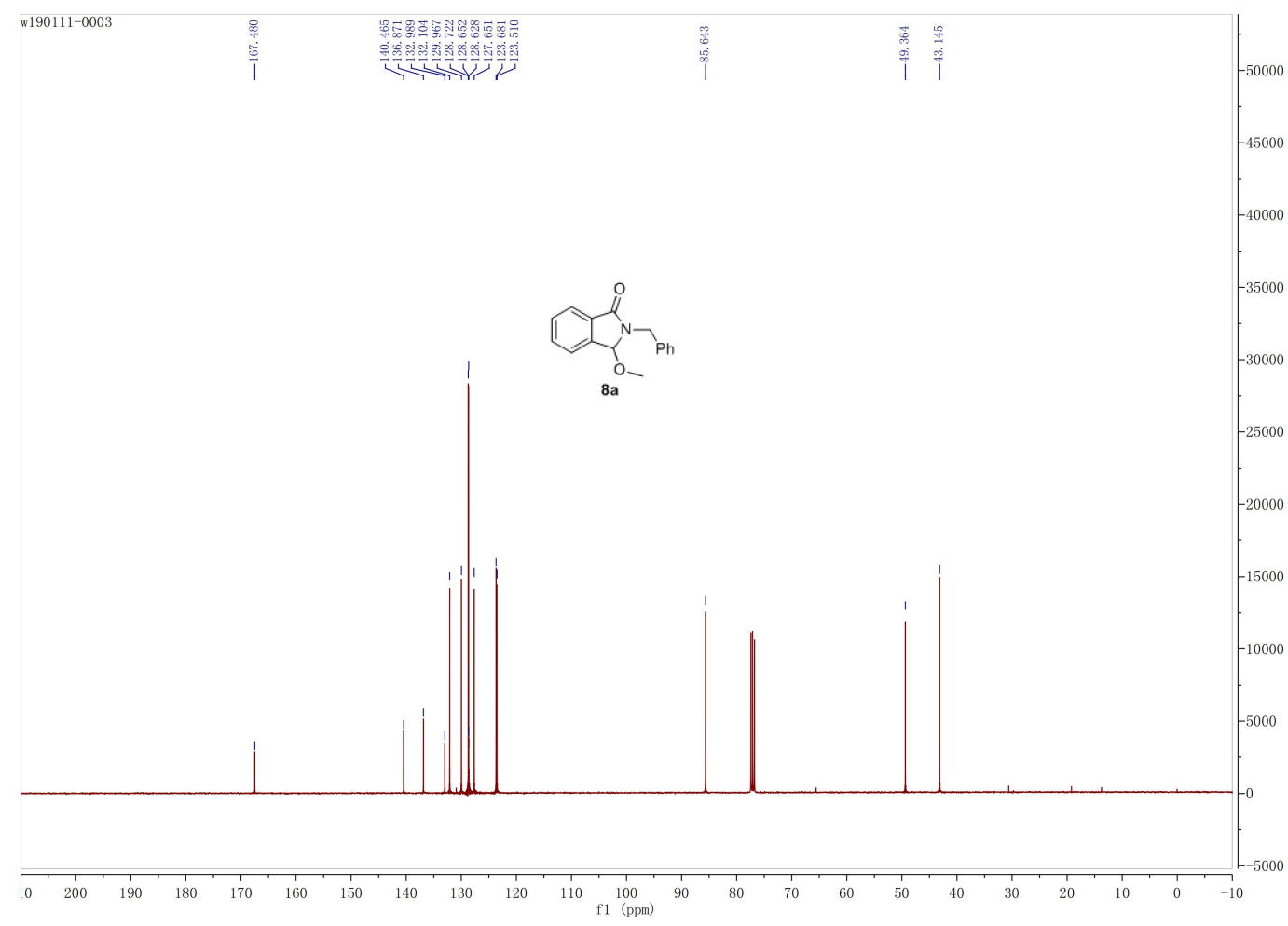

Figure S52. ${ }^{13} \mathrm{CNMR}\left(100 \mathrm{MHz}, \mathrm{CDCl}_{3}\right)$ of compound 8a 


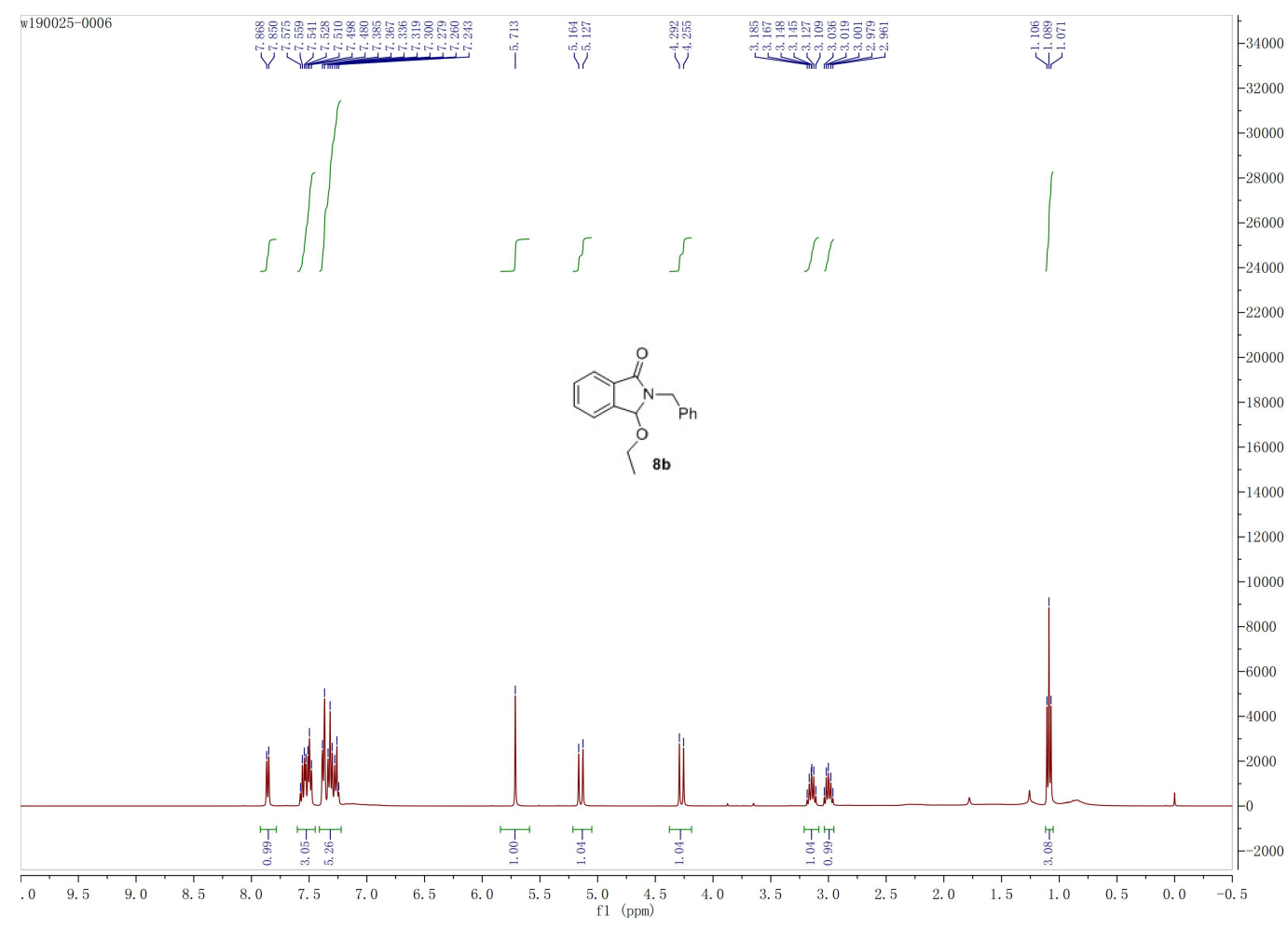

Figure S53. ${ }^{1} \mathrm{HNMR}\left(400 \mathrm{MHz}, \mathrm{CDCl}_{3}\right)$ of compound $8 \mathrm{~b}$

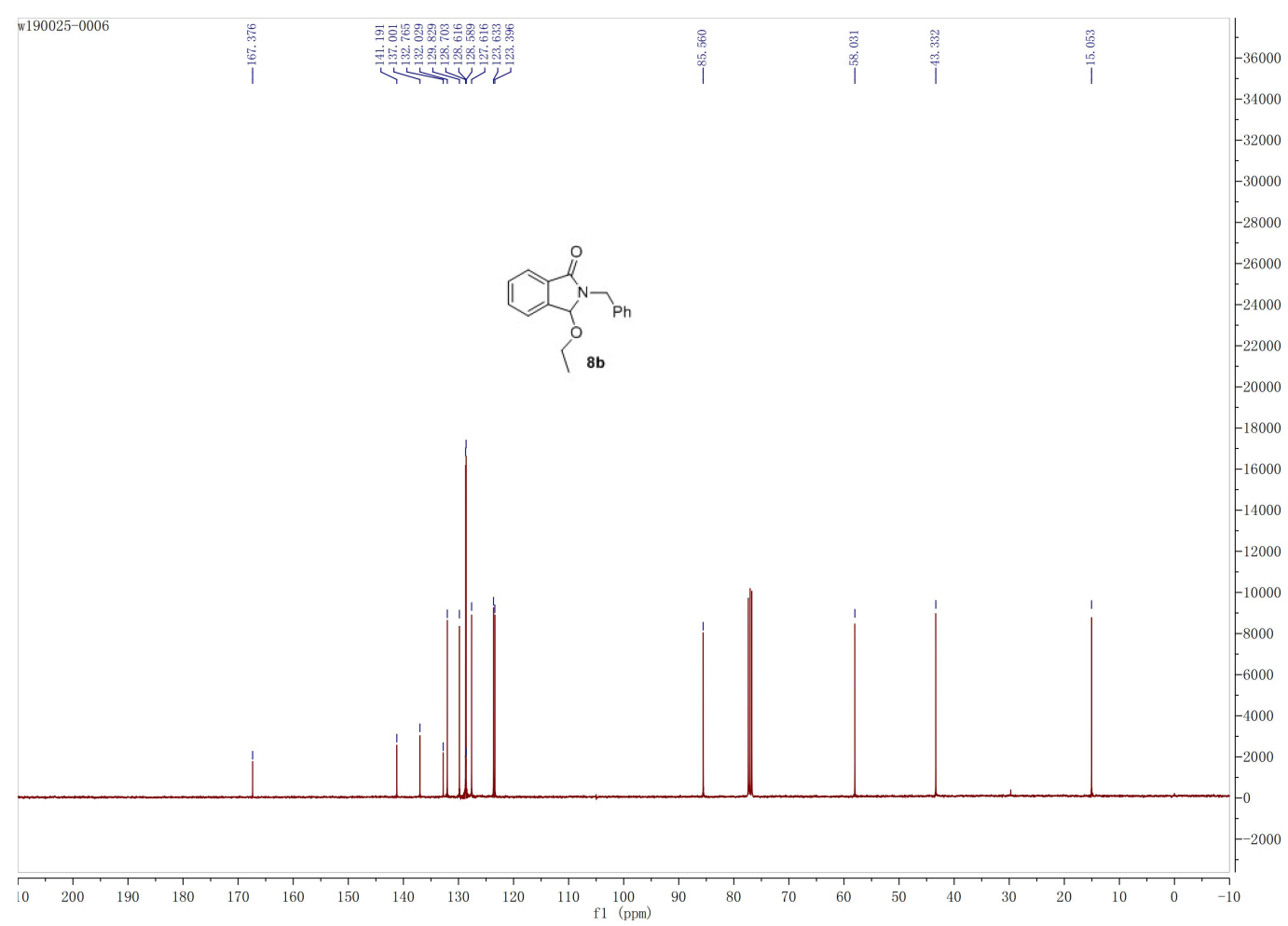

Figure S54. ${ }^{13} \mathrm{CNMR}\left(100 \mathrm{MHz}, \mathrm{CDCl}_{3}\right)$ of compound $8 \mathrm{~b}$ 


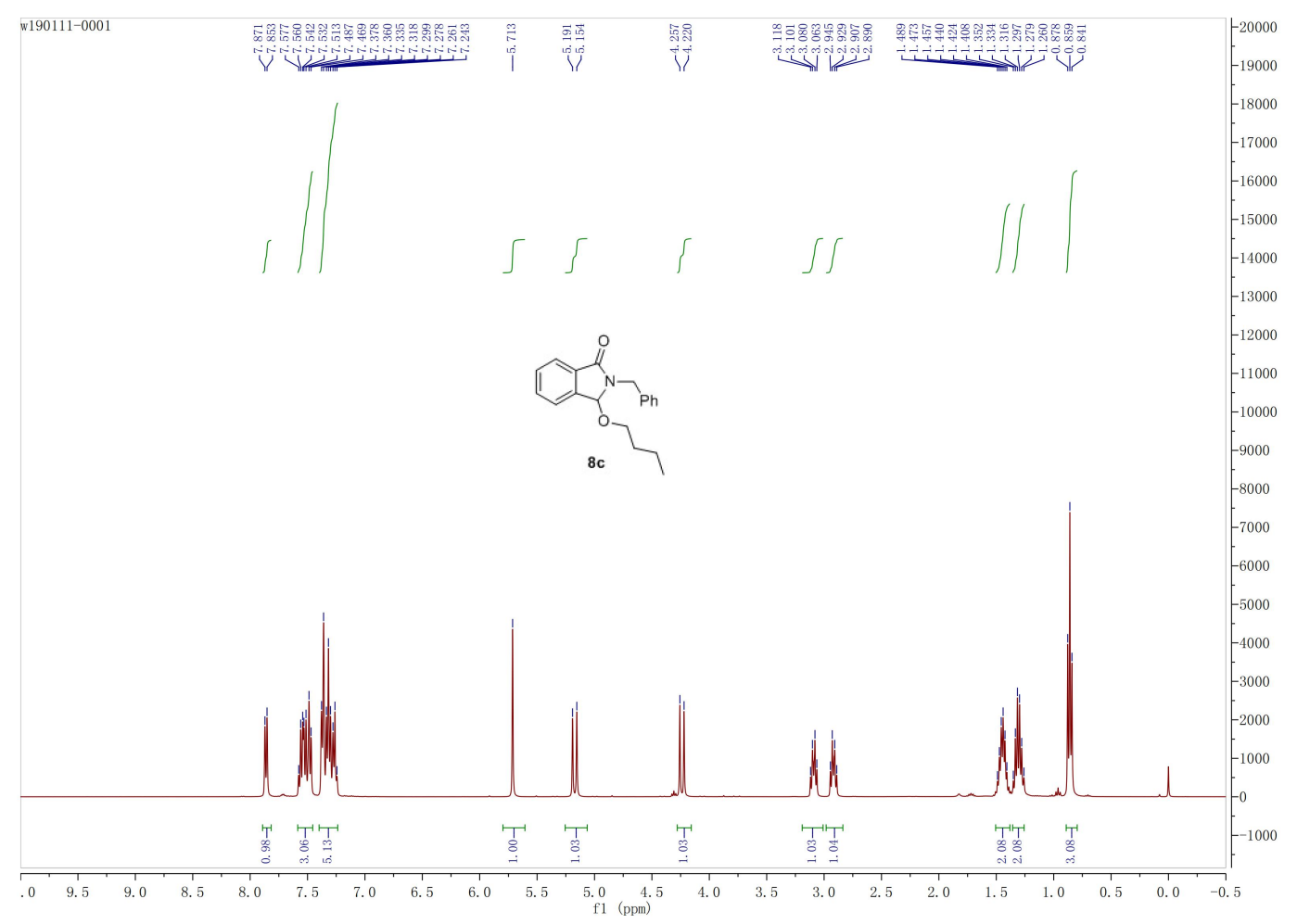

Figure S55. ${ }^{1} \mathrm{HNMR}\left(400 \mathrm{MHz}, \mathrm{CDCl}_{3}\right)$ of compound $8 \mathrm{c}$

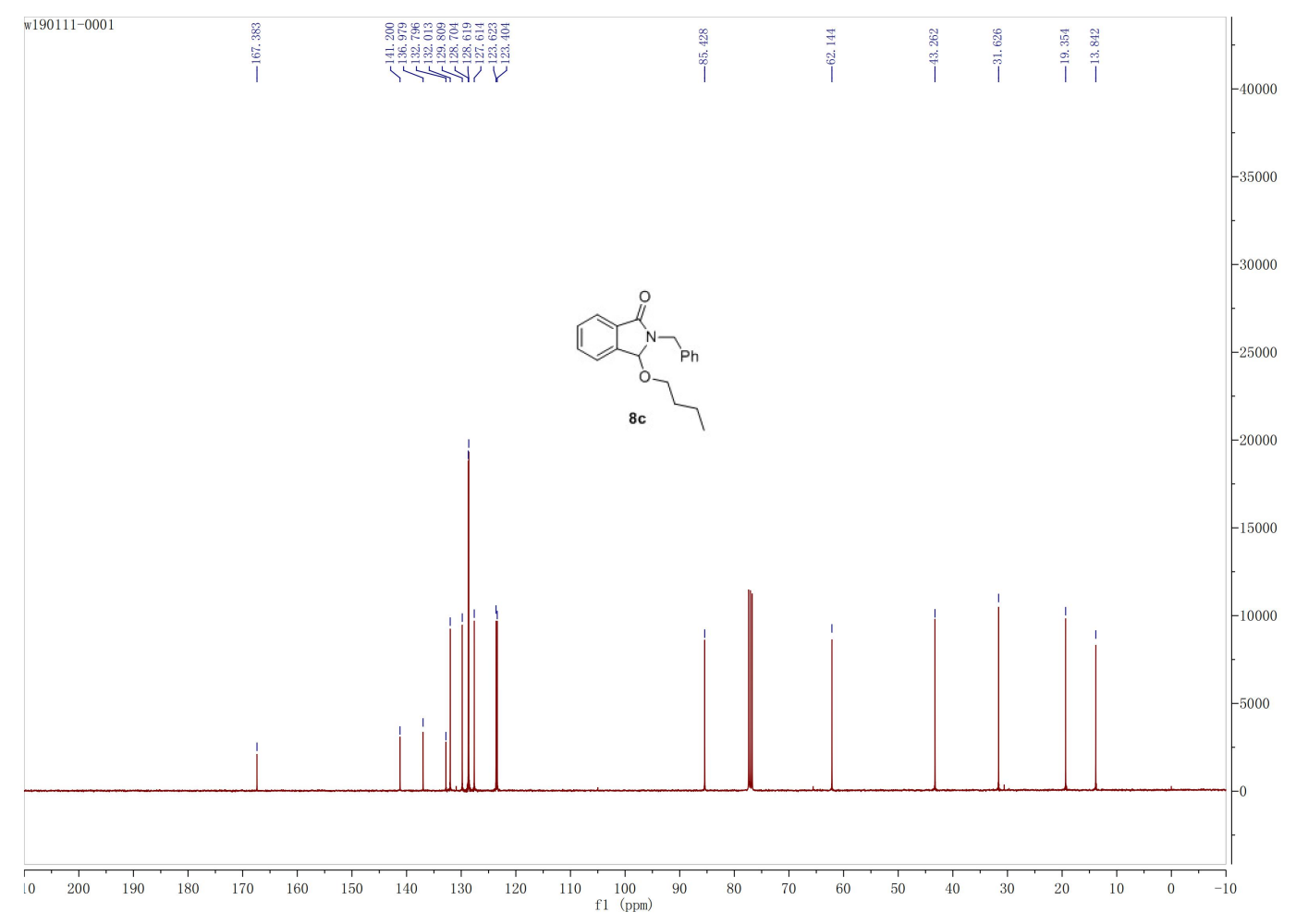

Figure S56. ${ }^{13} \mathrm{CNMR}\left(100 \mathrm{MHz}, \mathrm{CDCl}_{3}\right)$ of compound $8 \mathrm{c}$ 


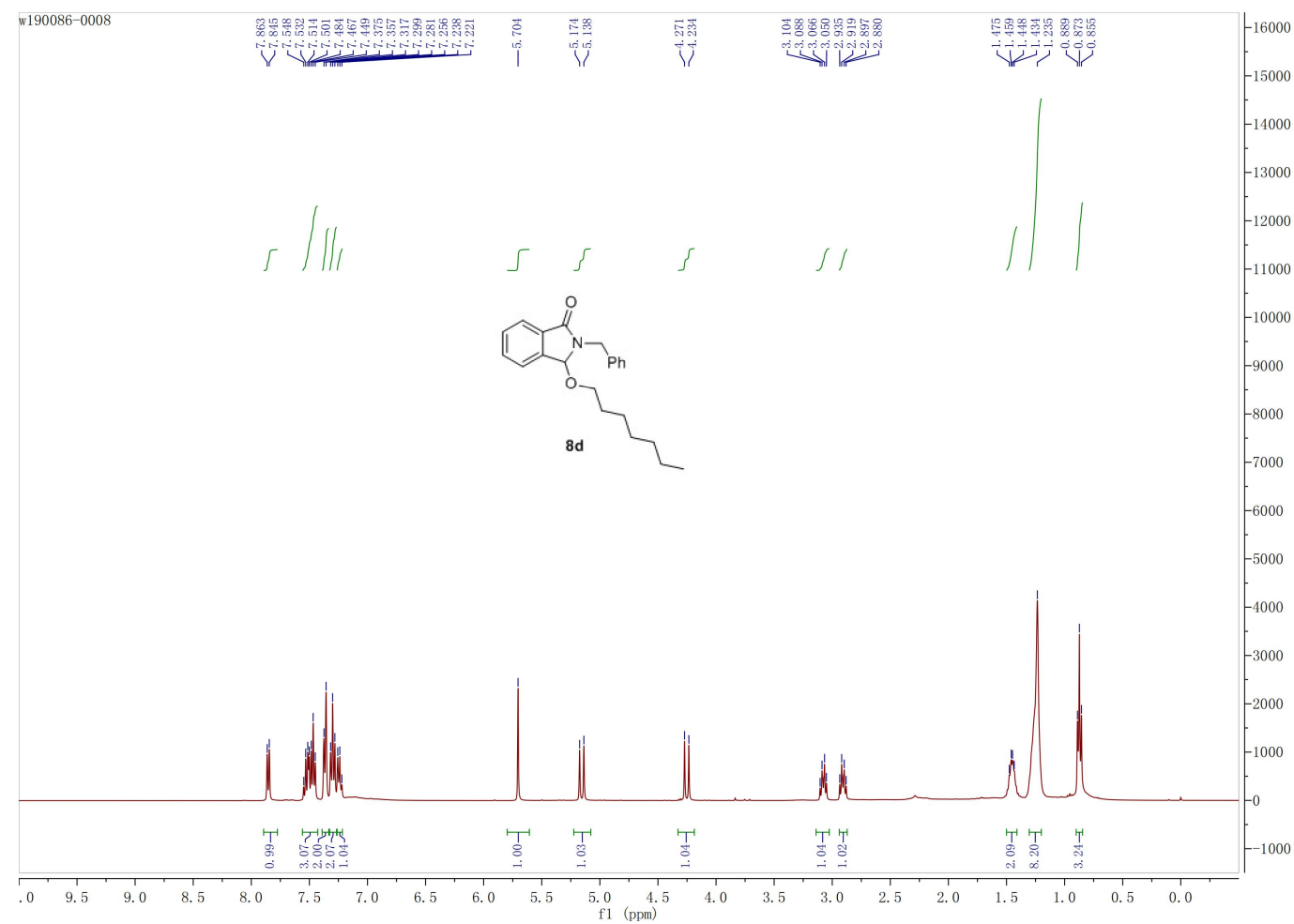

Figure S57. ${ }^{1} \mathrm{HNMR}\left(400 \mathrm{MHz}, \mathrm{CDCl}_{3}\right)$ of compound 8d

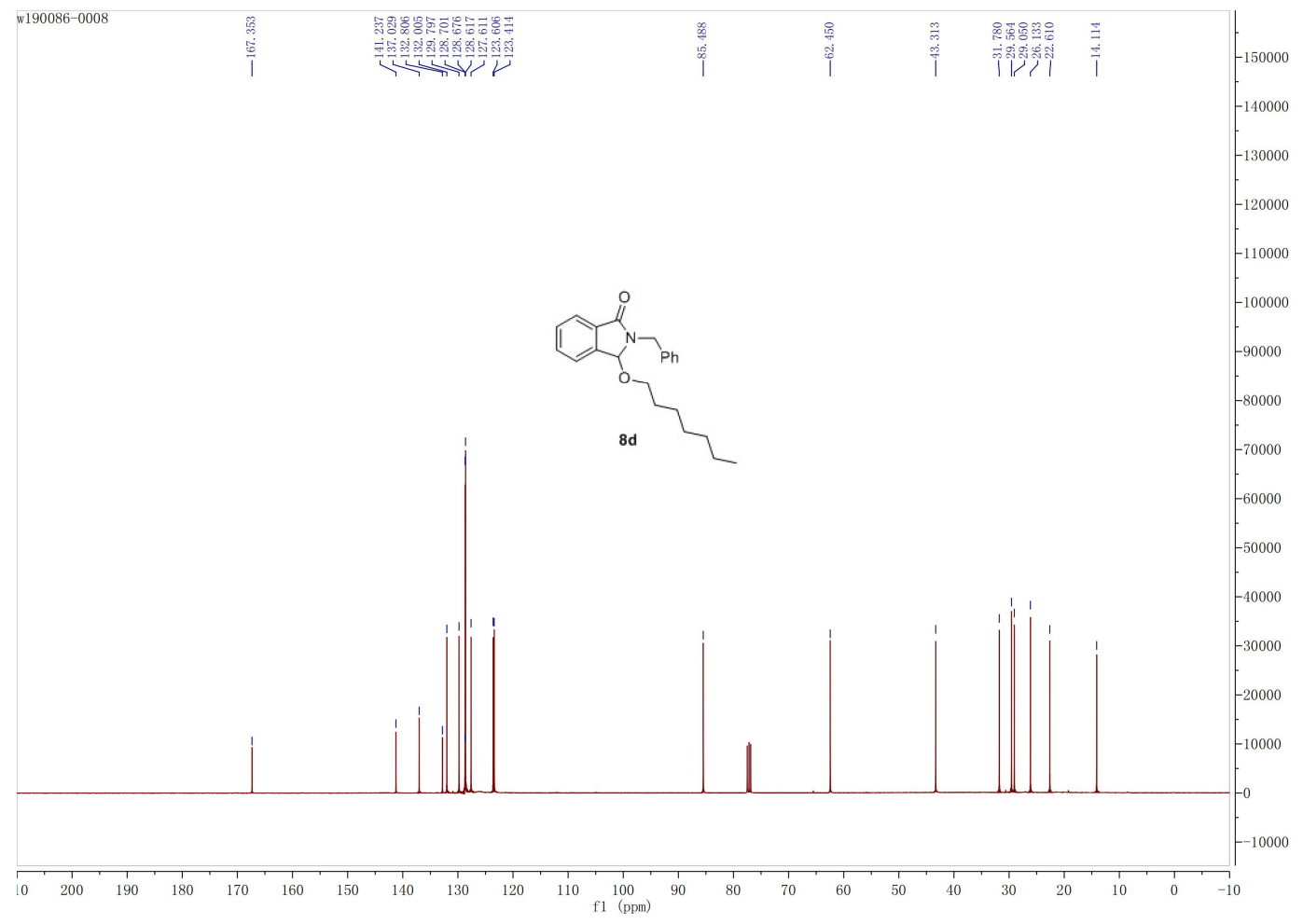

Figure S58. ${ }^{13} \mathrm{CNMR}\left(100 \mathrm{MHz}, \mathrm{CDCl}_{3}\right)$ of compound 8d 


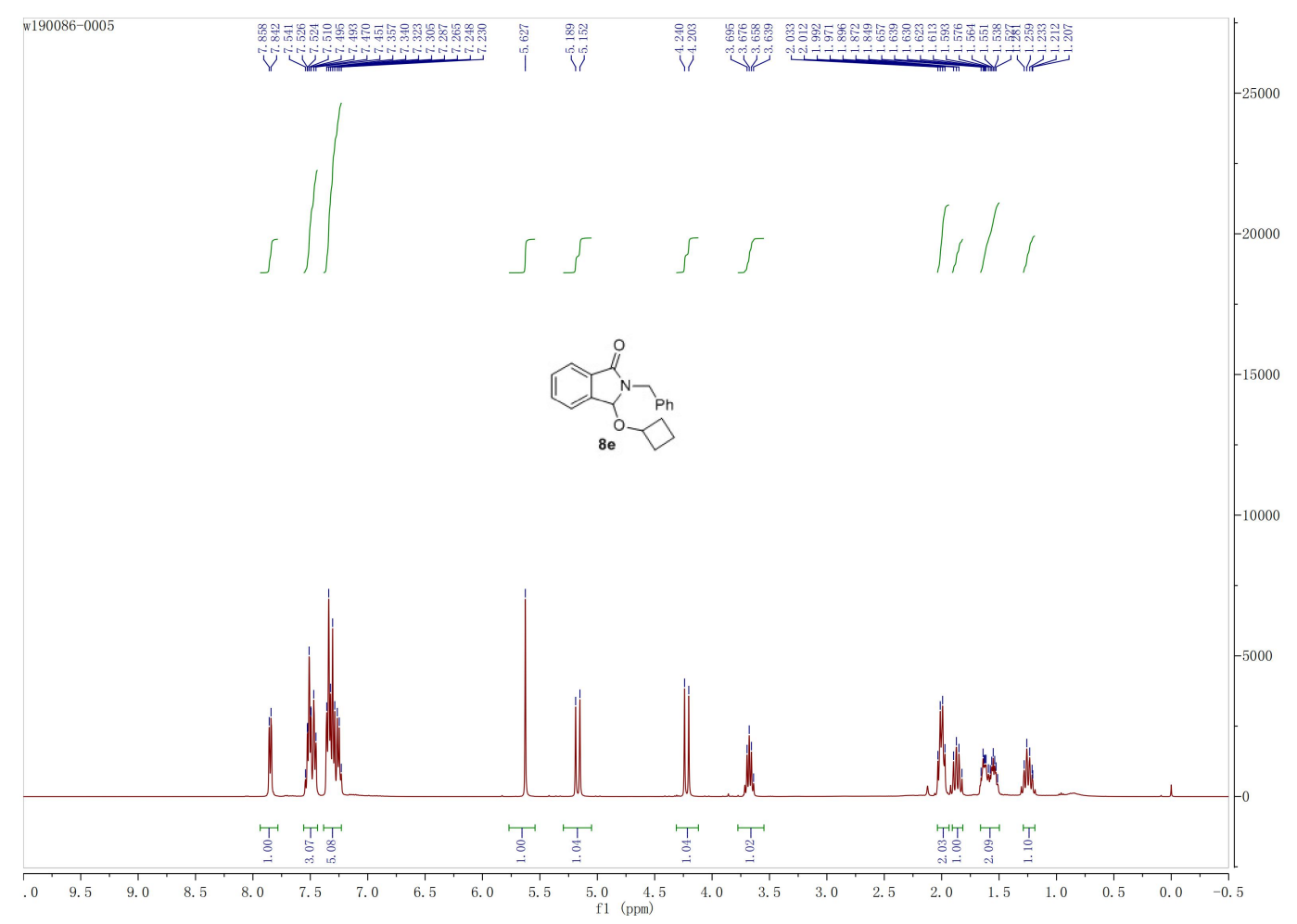

Figure S59. ${ }^{1} \mathrm{HNMR}\left(400 \mathrm{MHz}, \mathrm{CDCl}_{3}\right)$ of compound $8 \mathrm{e}$

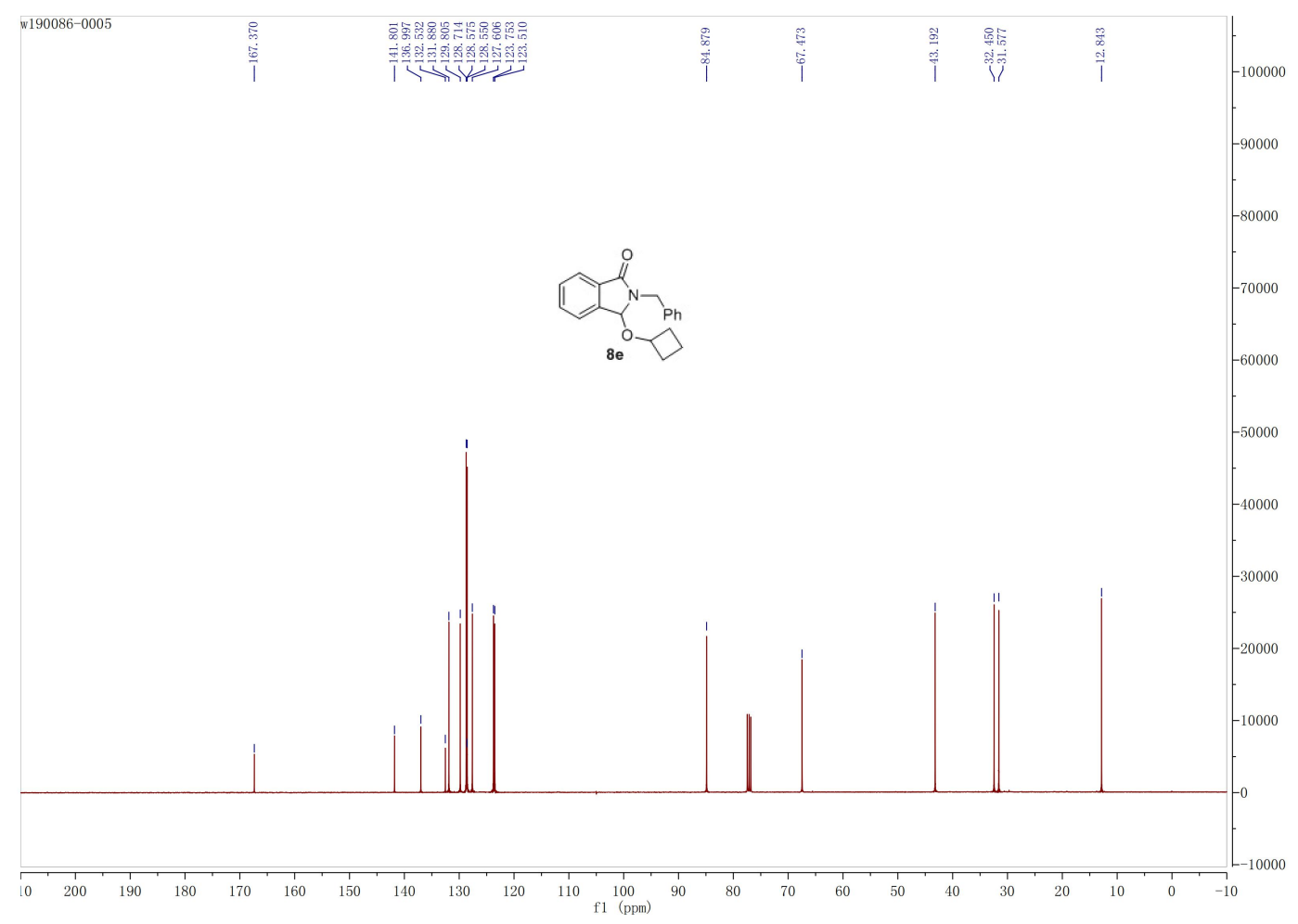

Figure S60. ${ }^{13} \mathrm{CNMR}\left(100 \mathrm{MHz}, \mathrm{CDCl}_{3}\right)$ of compound $8 \mathrm{e}$ 


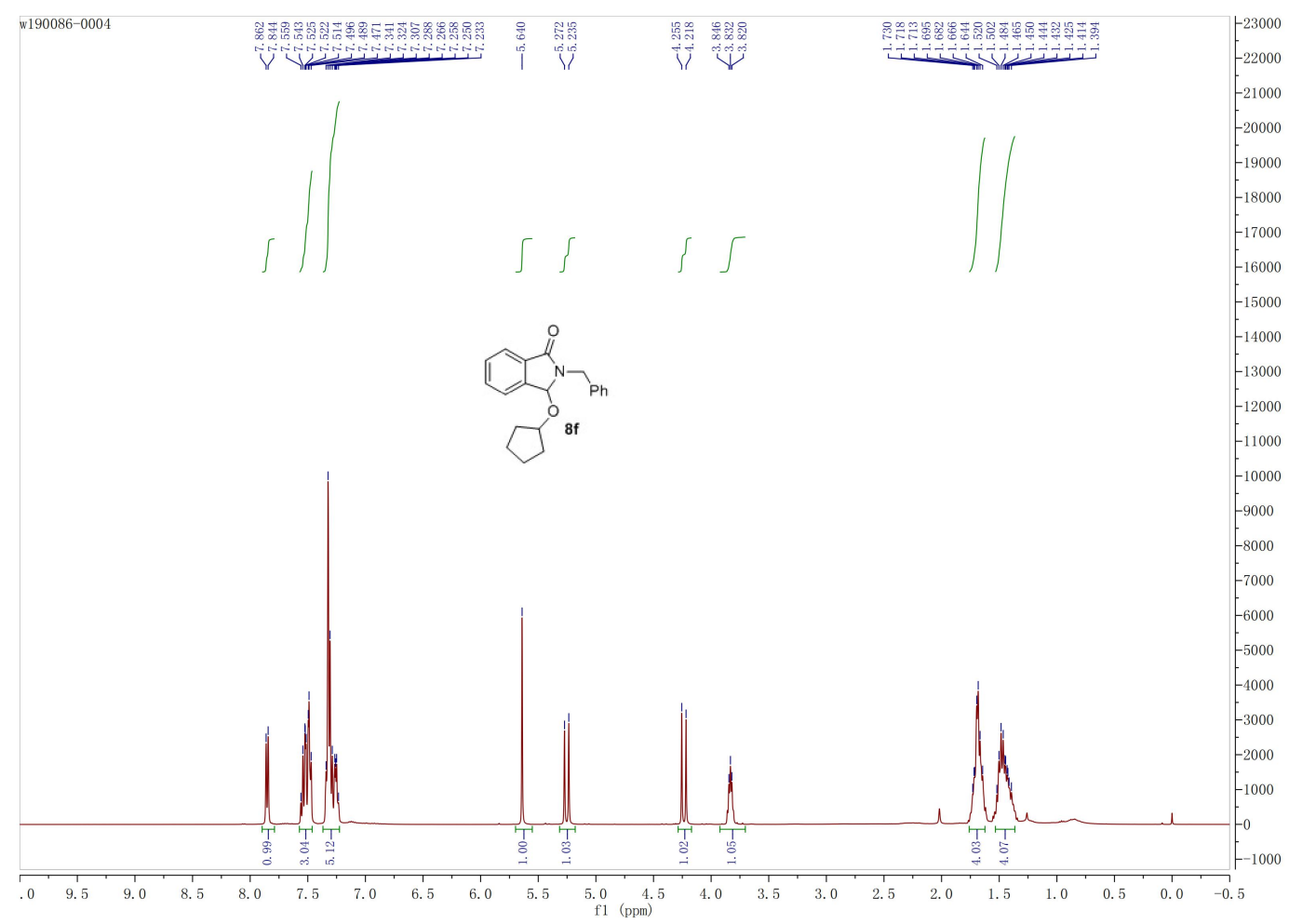

Figure S61. ${ }^{1} \mathrm{HNMR}\left(400 \mathrm{MHz}, \mathrm{CDCl}_{3}\right)$ of compound $8 \mathrm{f}$

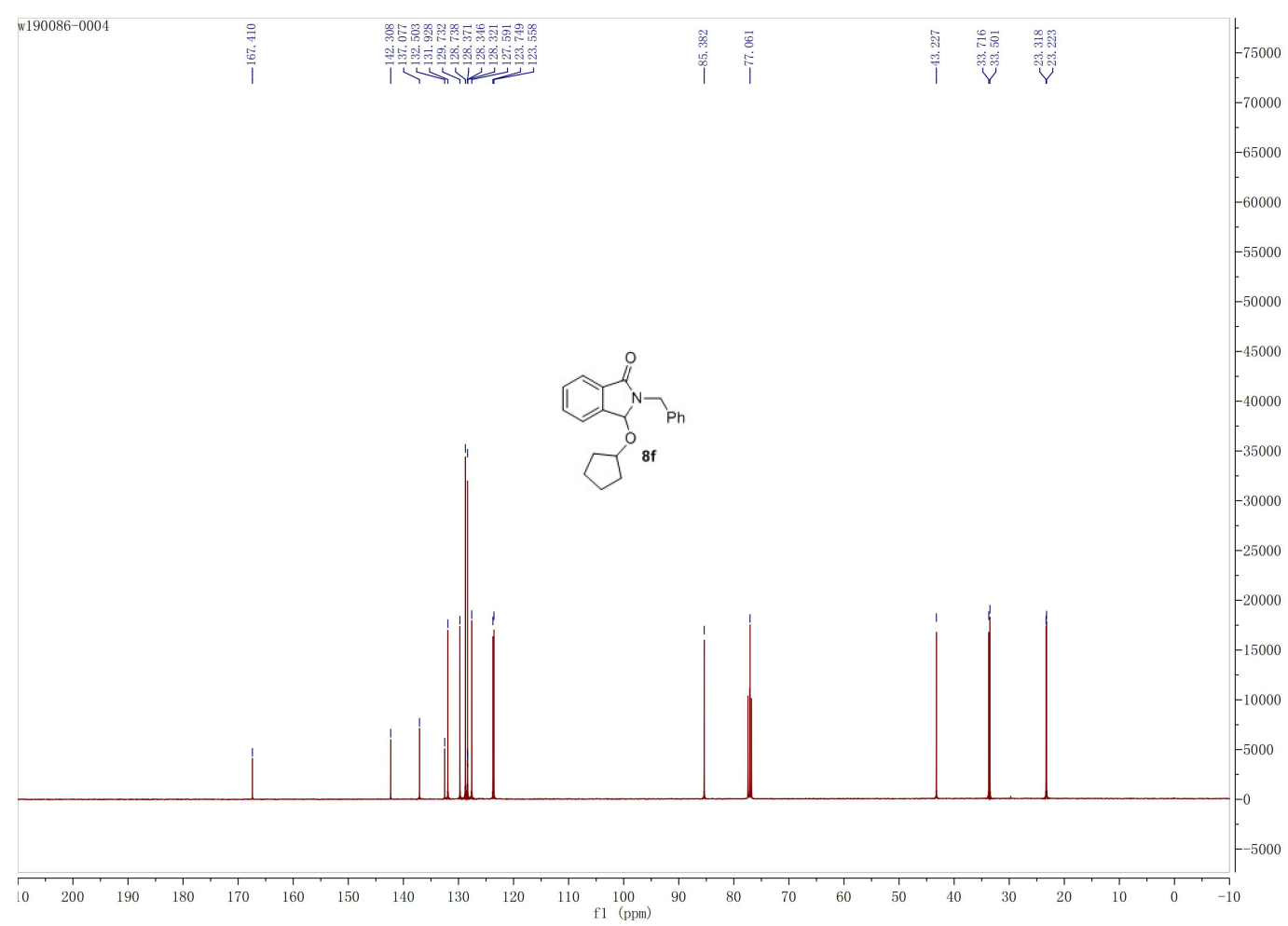

Figure S62. ${ }^{13} \mathrm{CNMR}\left(100 \mathrm{MHz}, \mathrm{CDCl}_{3}\right)$ of compound $8 \mathrm{f}$ 


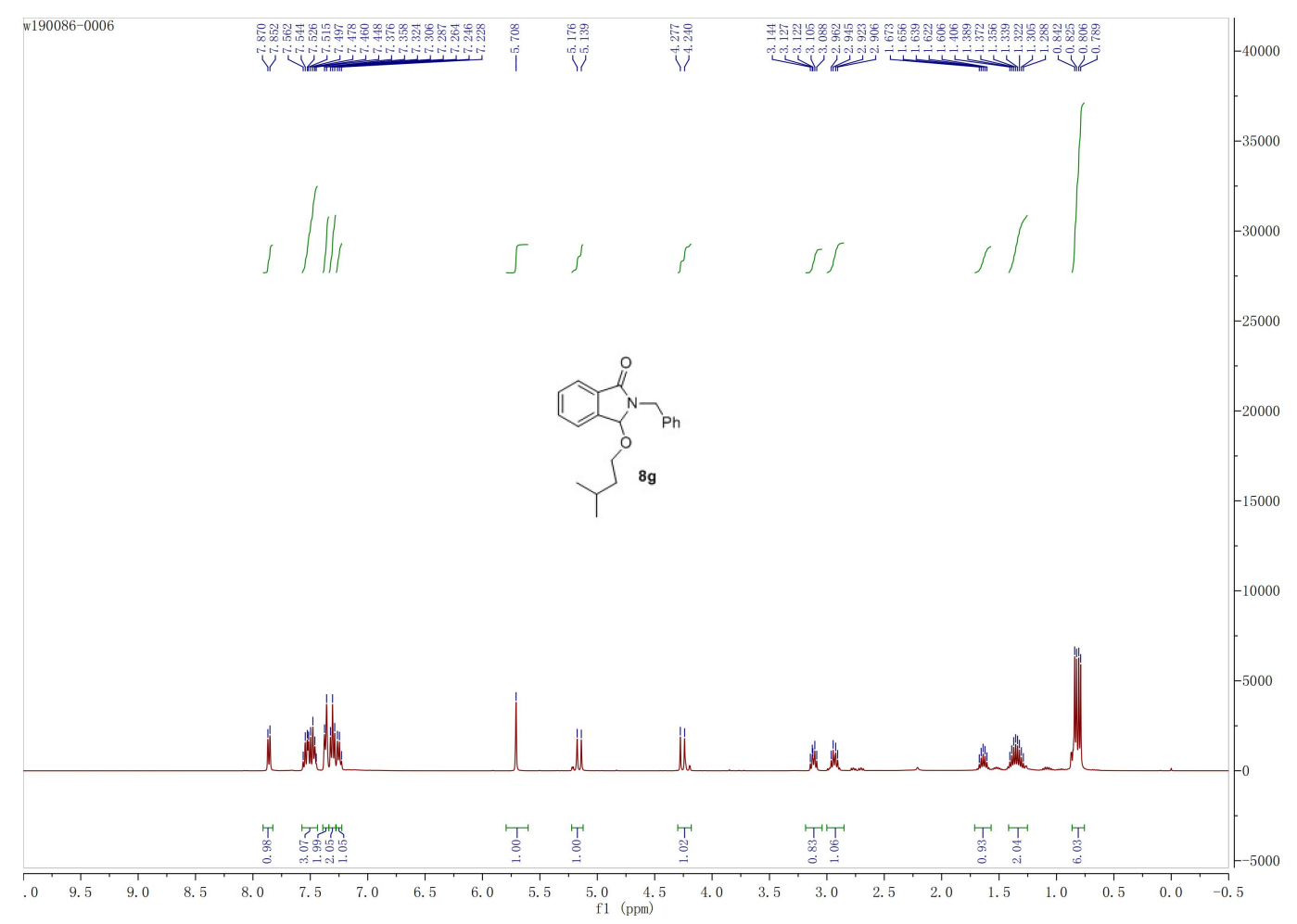

Figure S63. ${ }^{1} \mathrm{HNMR}\left(400 \mathrm{MHz}, \mathrm{CDCl}_{3}\right)$ of compound $8 \mathrm{~g}$

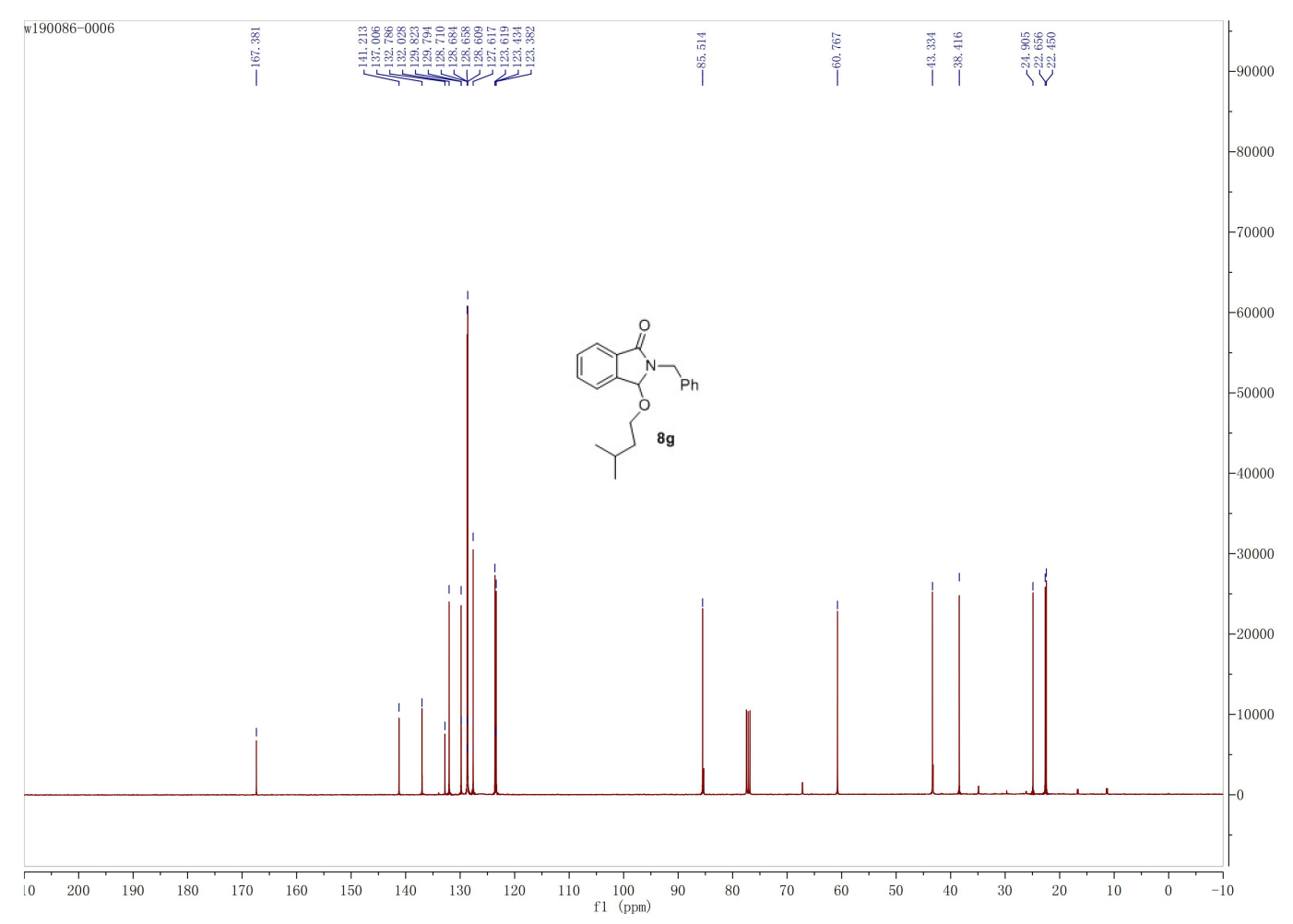

Figure S64. ${ }^{13} \mathrm{CNMR}\left(100 \mathrm{MHz}, \mathrm{CDCl}_{3}\right)$ of compound $8 \mathrm{~g}$ 


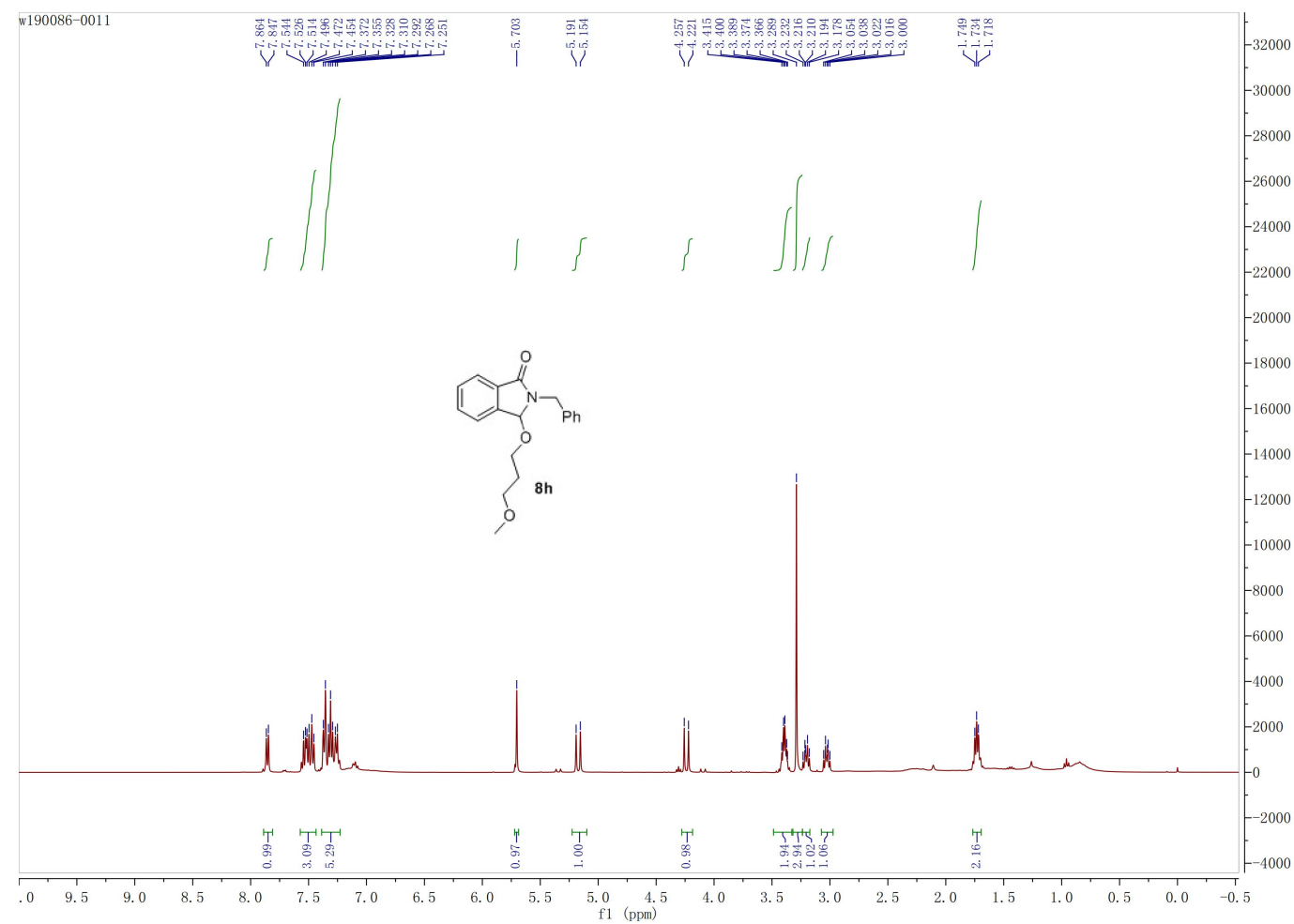

Figure S65. ${ }^{1} \mathrm{HNMR}\left(400 \mathrm{MHz}, \mathrm{CDCl}_{3}\right)$ of compound $8 \mathrm{~h}$

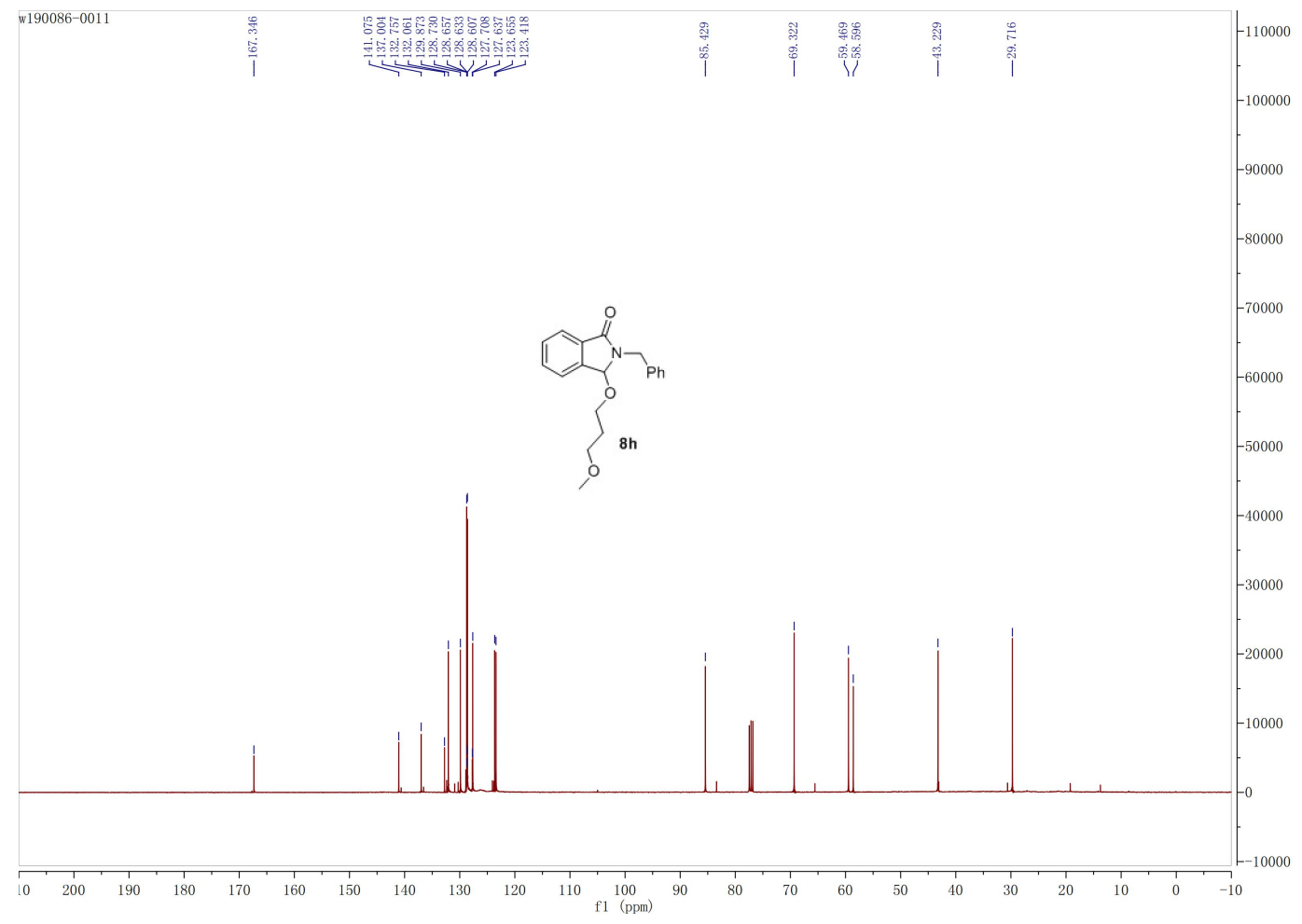

Figure S66. ${ }^{13} \mathrm{CNMR}\left(100 \mathrm{MHz}, \mathrm{CDCl}_{3}\right)$ of compound $8 \mathrm{~h}$ 


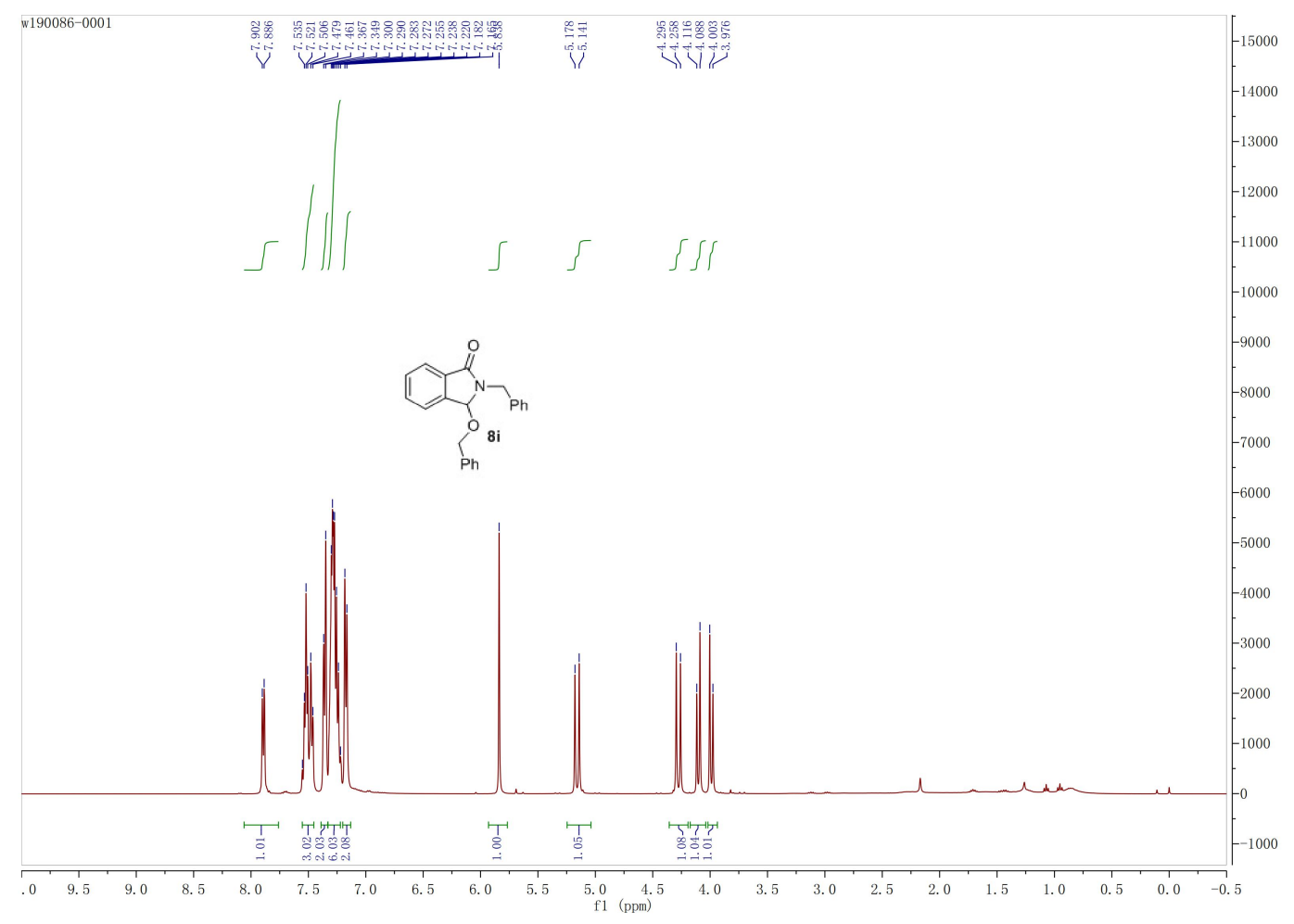

Figure S67. ${ }^{1} \mathrm{HNMR}\left(400 \mathrm{MHz}, \mathrm{CDCl}_{3}\right)$ of compound $8 \mathrm{i}$

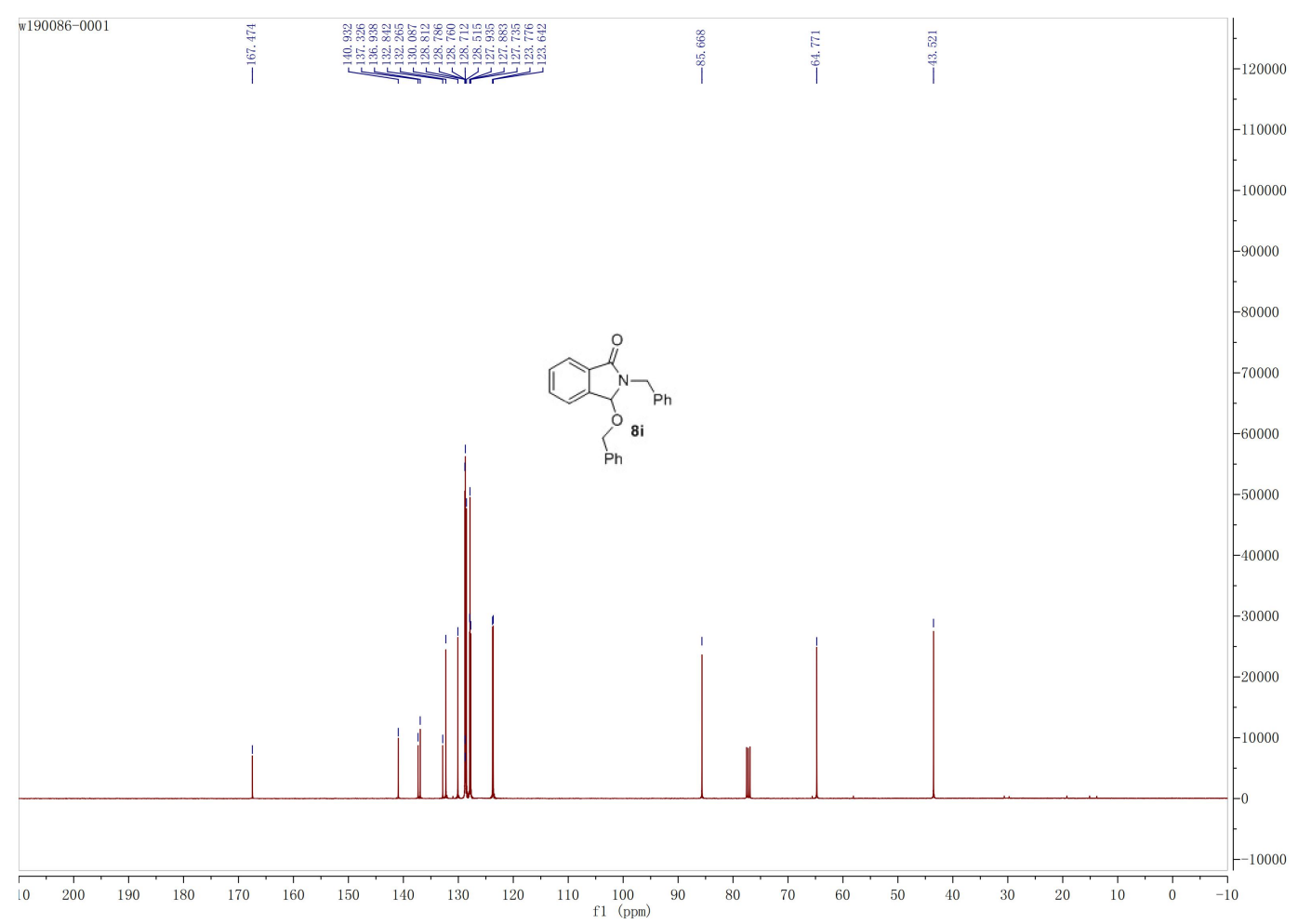

Figure S68. ${ }^{13} \mathrm{CNMR}\left(100 \mathrm{MHz}, \mathrm{CDCl}_{3}\right)$ of compound $8 \mathrm{i}$ 


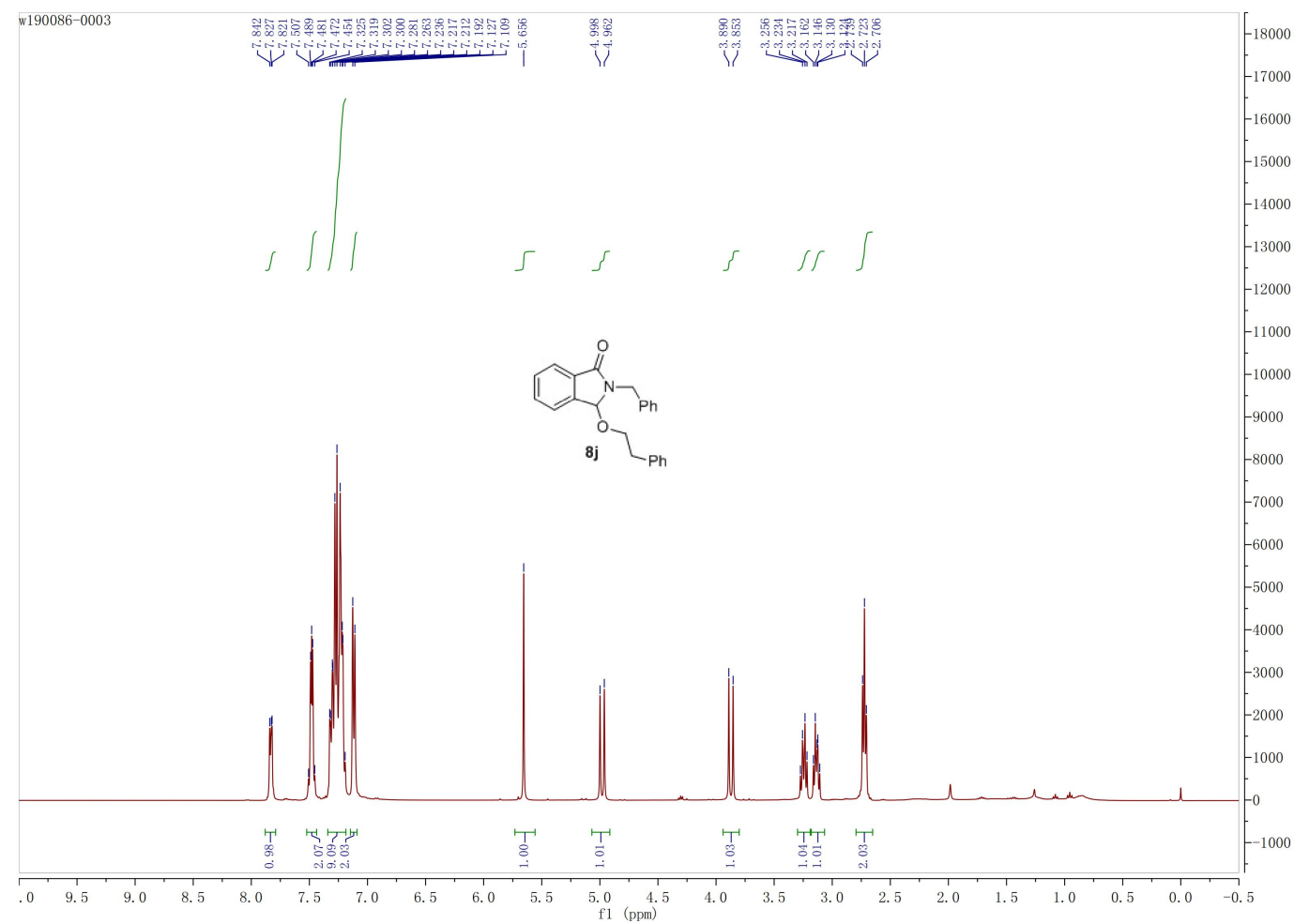

Figure S69. ${ }^{1} \mathrm{HNMR}\left(400 \mathrm{MHz}, \mathrm{CDCl}_{3}\right)$ of compound $8 \mathrm{j}$

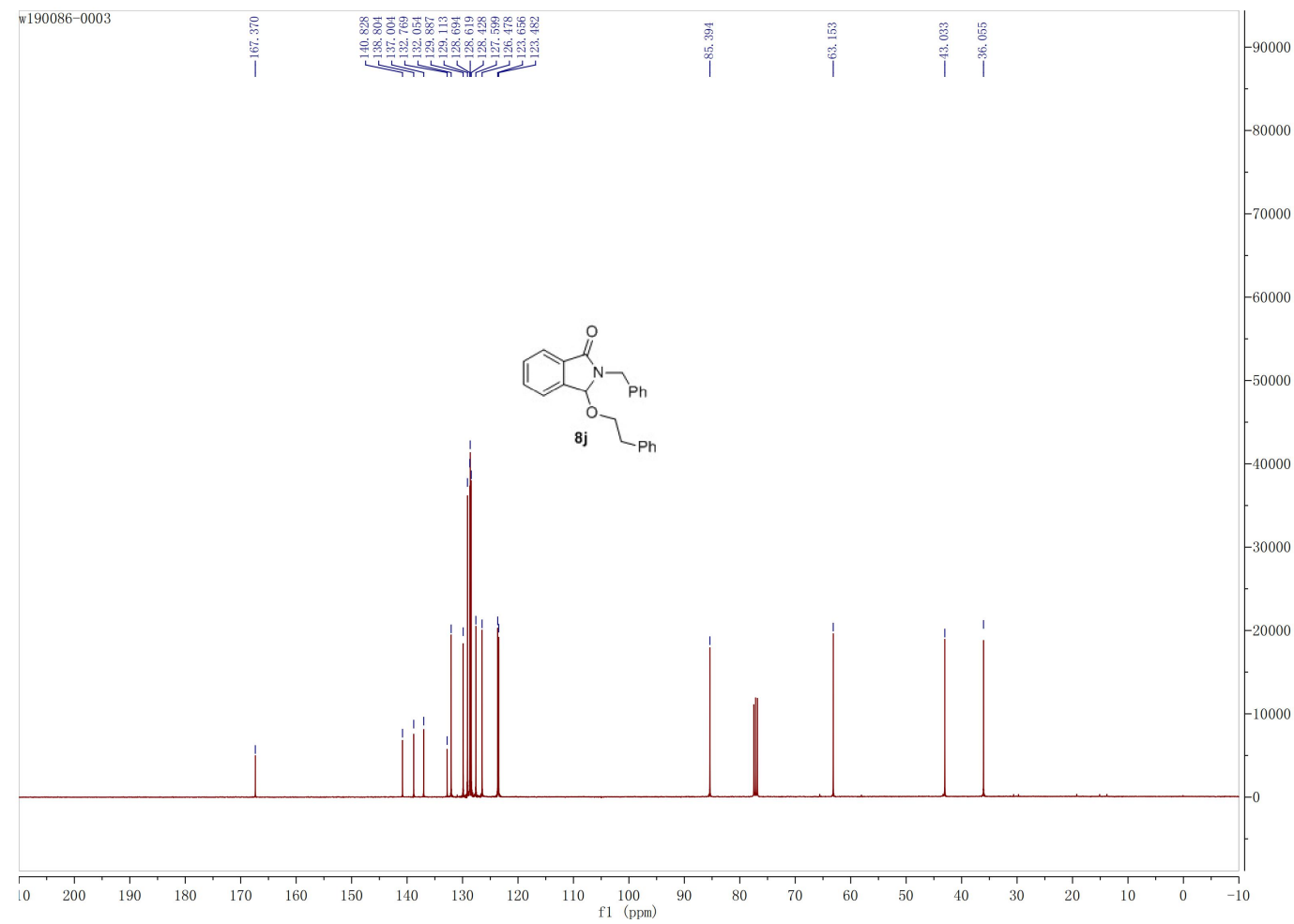

Figure S70. ${ }^{13} \mathrm{CNMR}\left(100 \mathrm{MHz}, \mathrm{CDCl}_{3}\right)$ of compound 8j 


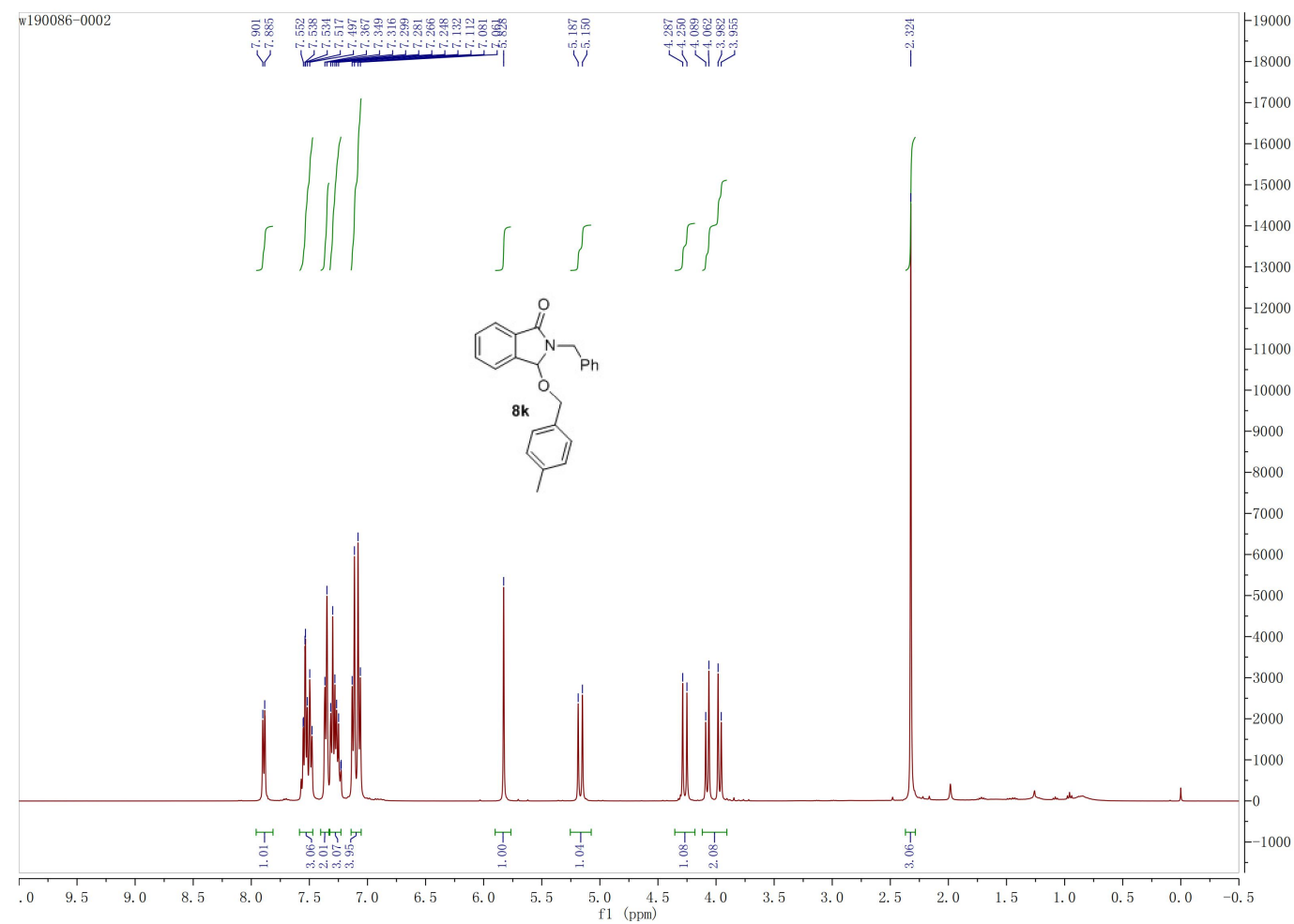

Figure S71. ${ }^{1} \mathrm{HNMR}\left(400 \mathrm{MHz}, \mathrm{CDCl}_{3}\right)$ of compound $8 \mathrm{k}$

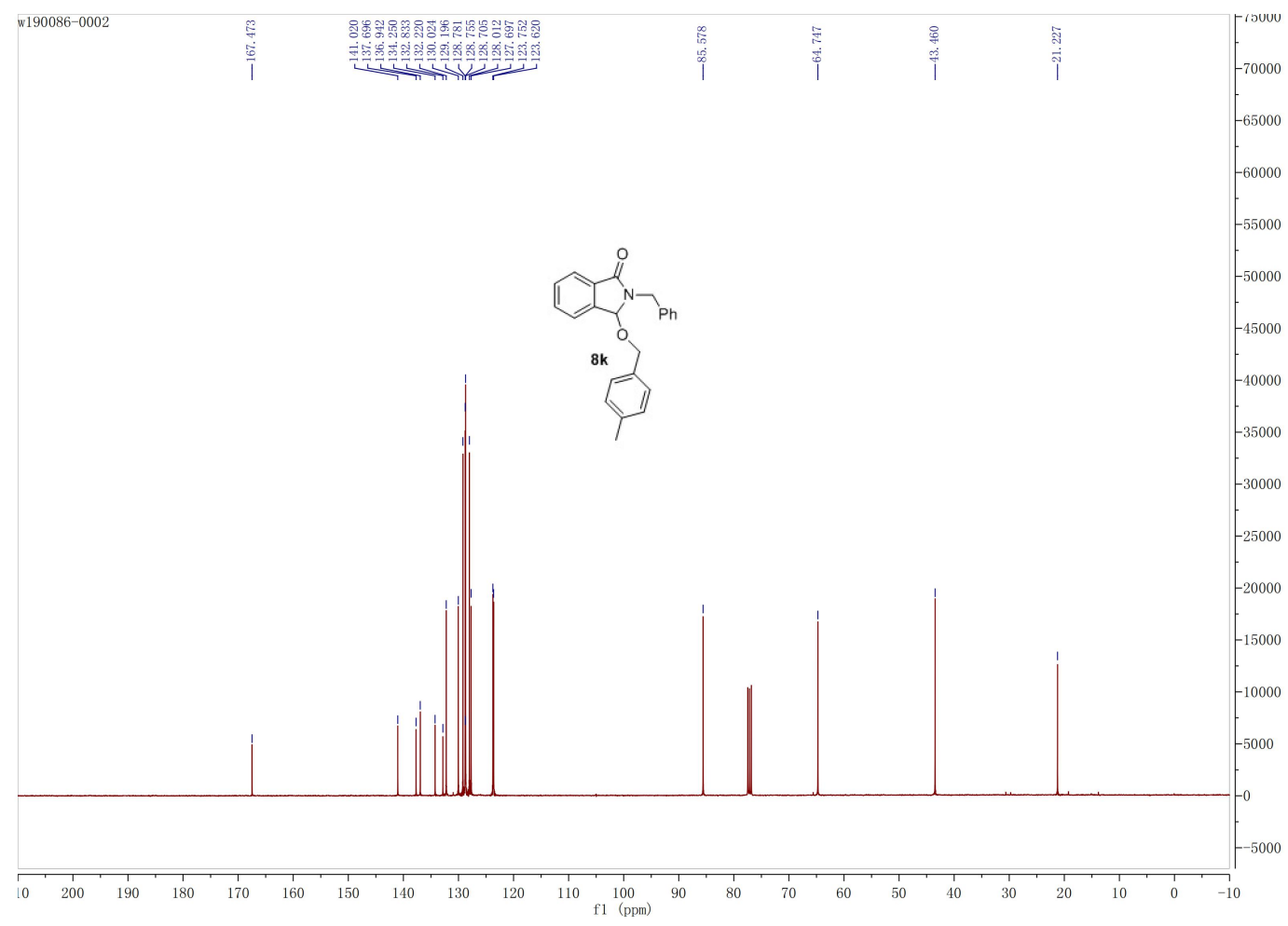

Figure S72. ${ }^{13} \mathrm{CNMR}\left(100 \mathrm{MHz}, \mathrm{CDCl}_{3}\right)$ of compound $8 \mathrm{k}$ 


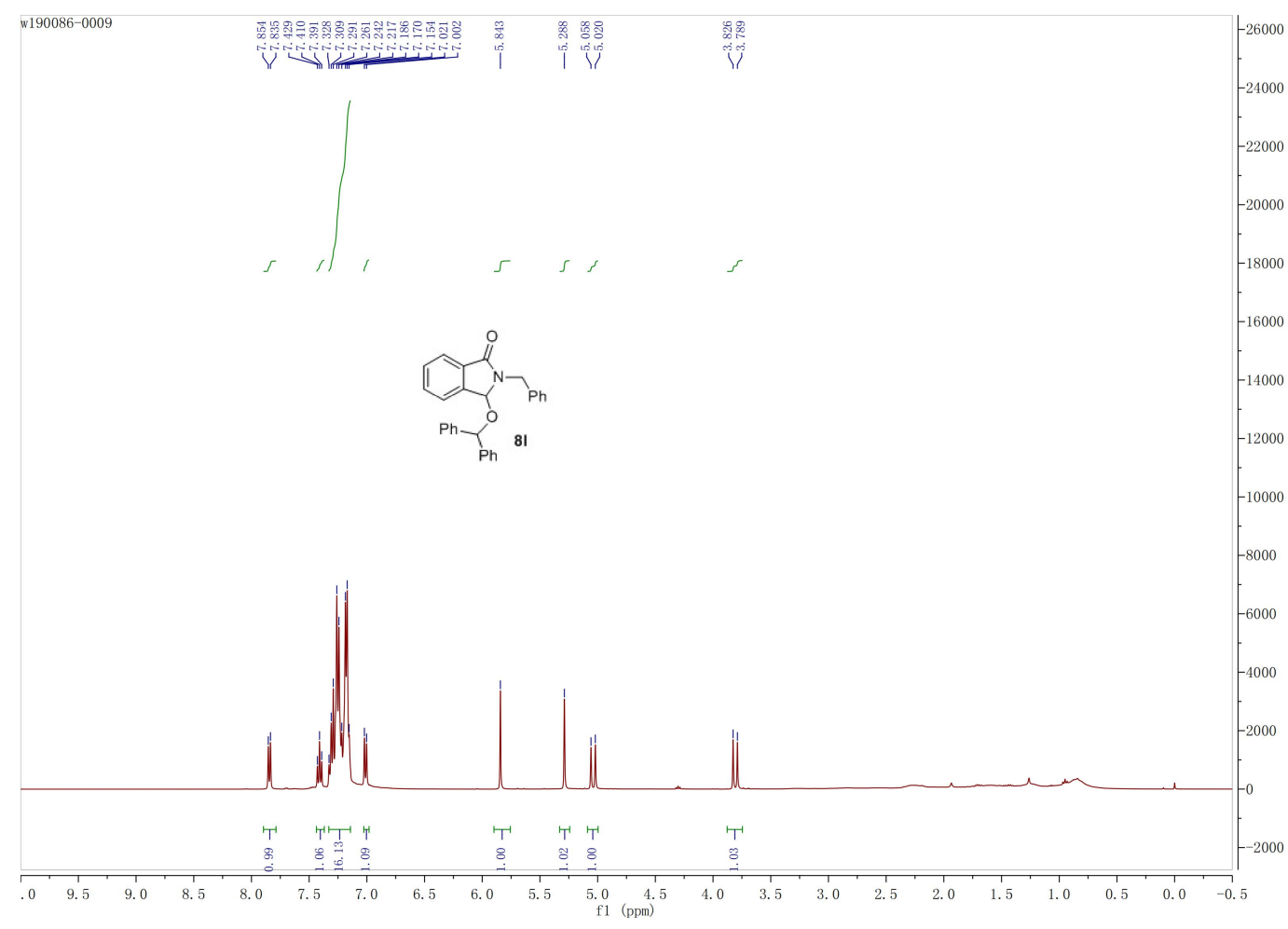

Figure S73. ${ }^{1} \mathrm{HNMR}\left(400 \mathrm{MHz}, \mathrm{CDCl}_{3}\right)$ of compound $8 \mathrm{I}$

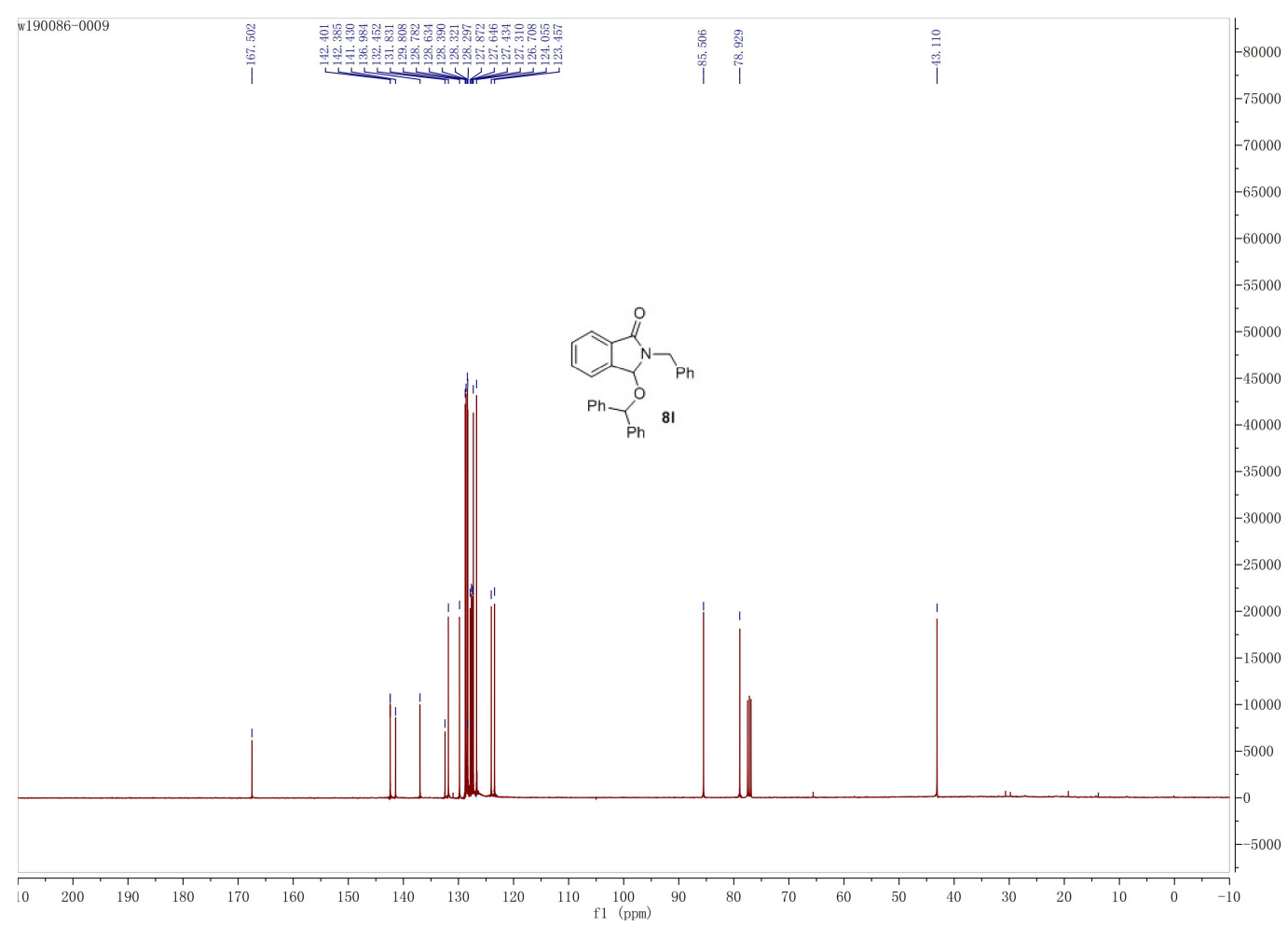

Figure S74. ${ }^{13} \mathrm{CNMR}\left(100 \mathrm{MHz}, \mathrm{CDCl}_{3}\right)$ of compound 81 


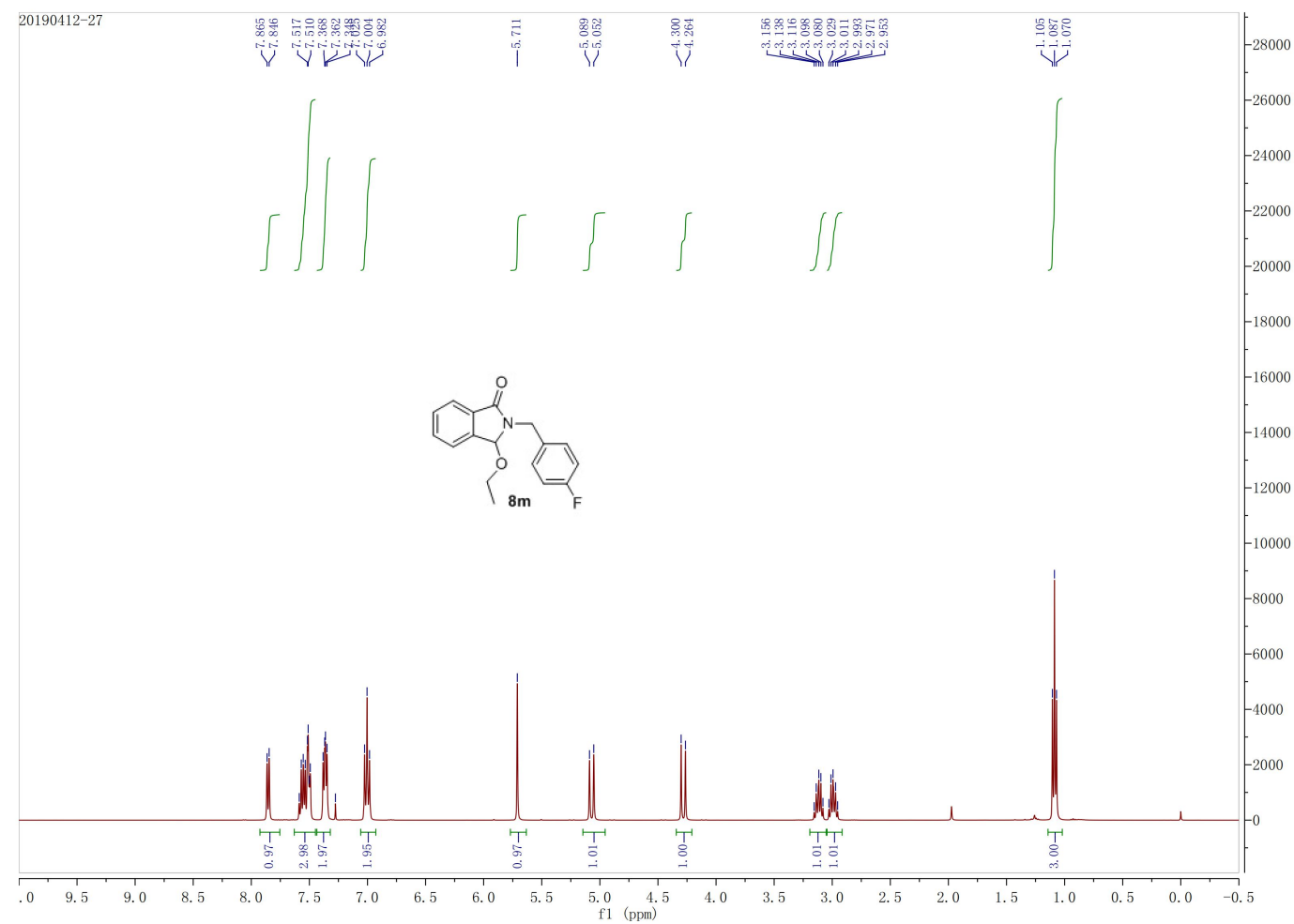

Figure S75. ${ }^{1} \mathrm{HNMR}\left(400 \mathrm{MHz}, \mathrm{CDCl}_{3}\right)$ of compound $8 \mathrm{~m}$

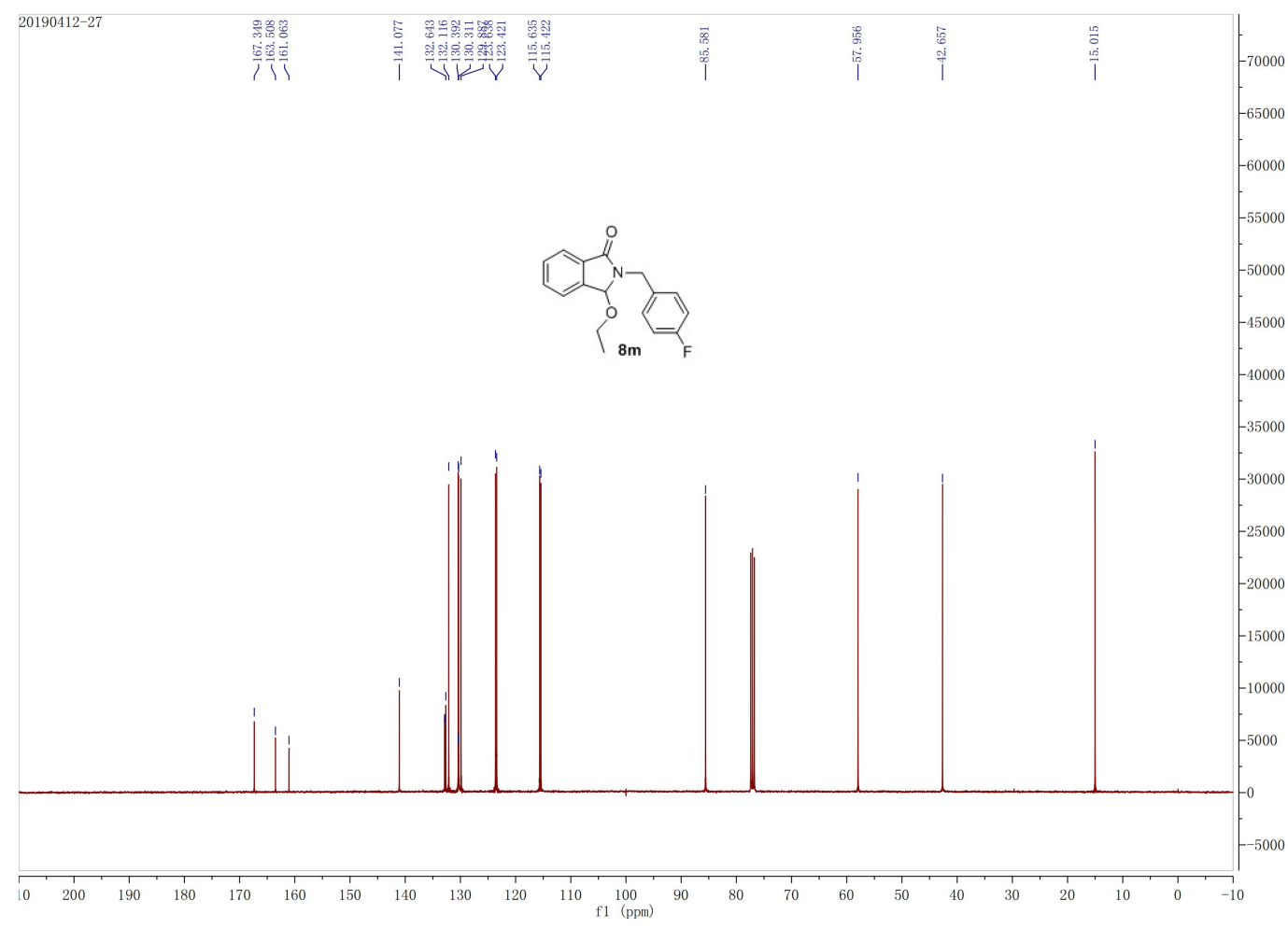

Figure S76. ${ }^{13} \mathrm{CNMR}\left(100 \mathrm{MHz}, \mathrm{CDCl}_{3}\right)$ of compound $8 \mathrm{~m}$ 


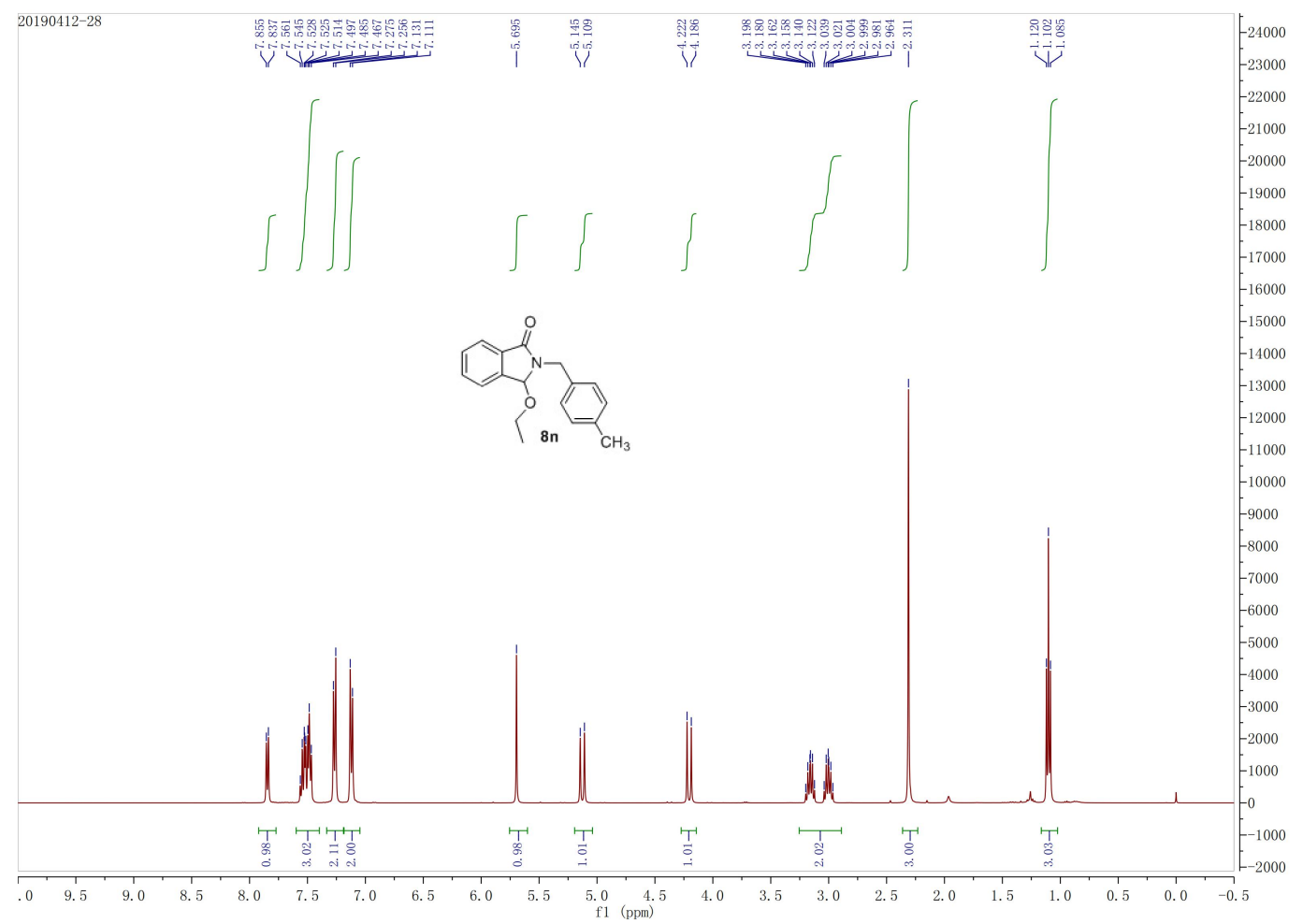

Figure S77. ${ }^{1} \mathrm{HNMR}\left(400 \mathrm{MHz}, \mathrm{CDCl}_{3}\right)$ of compound $8 \mathrm{n}$

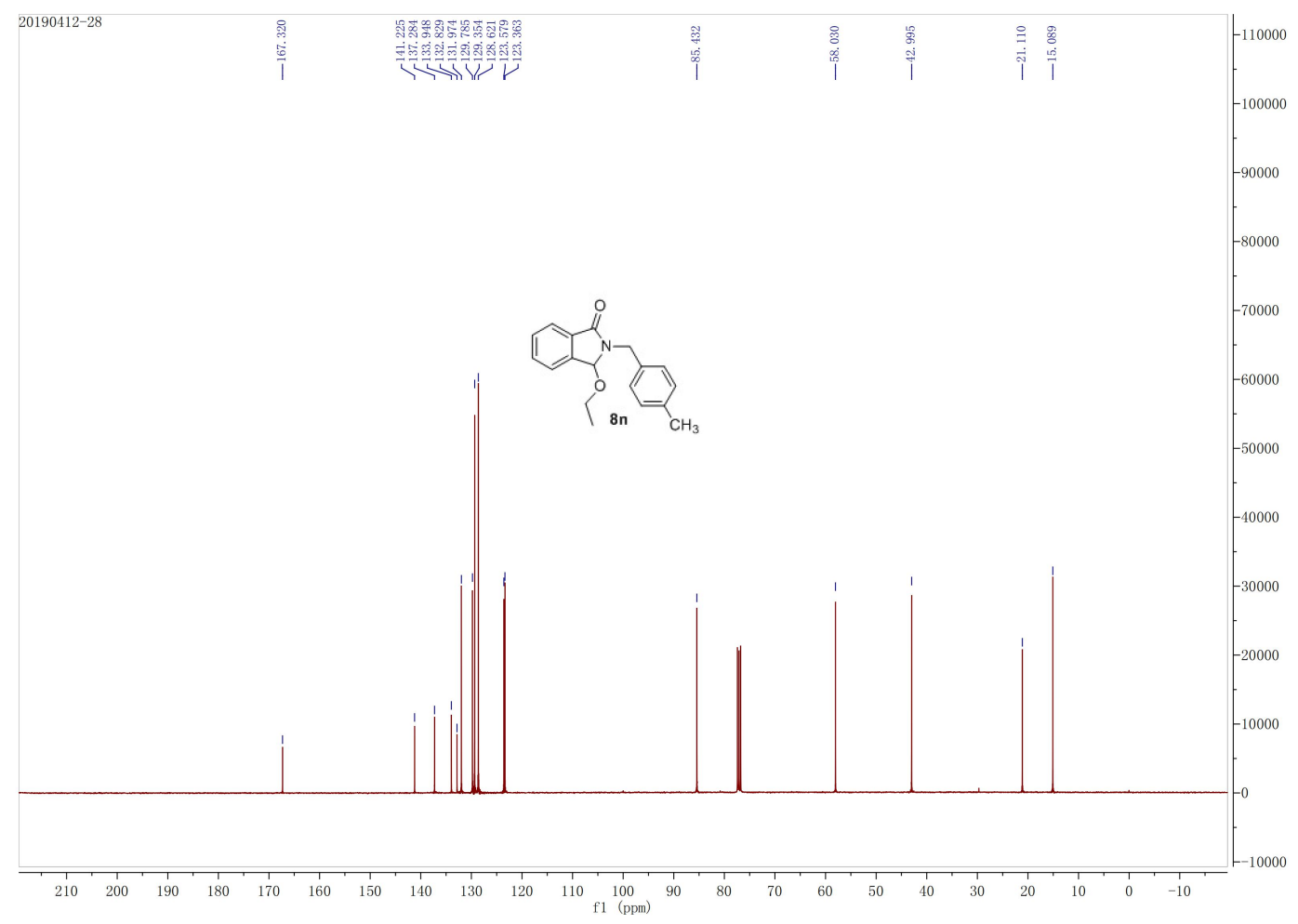

Figure S78. ${ }^{13} \mathrm{CNMR}\left(100 \mathrm{MHz}, \mathrm{CDCl}_{3}\right)$ of compound 8n 


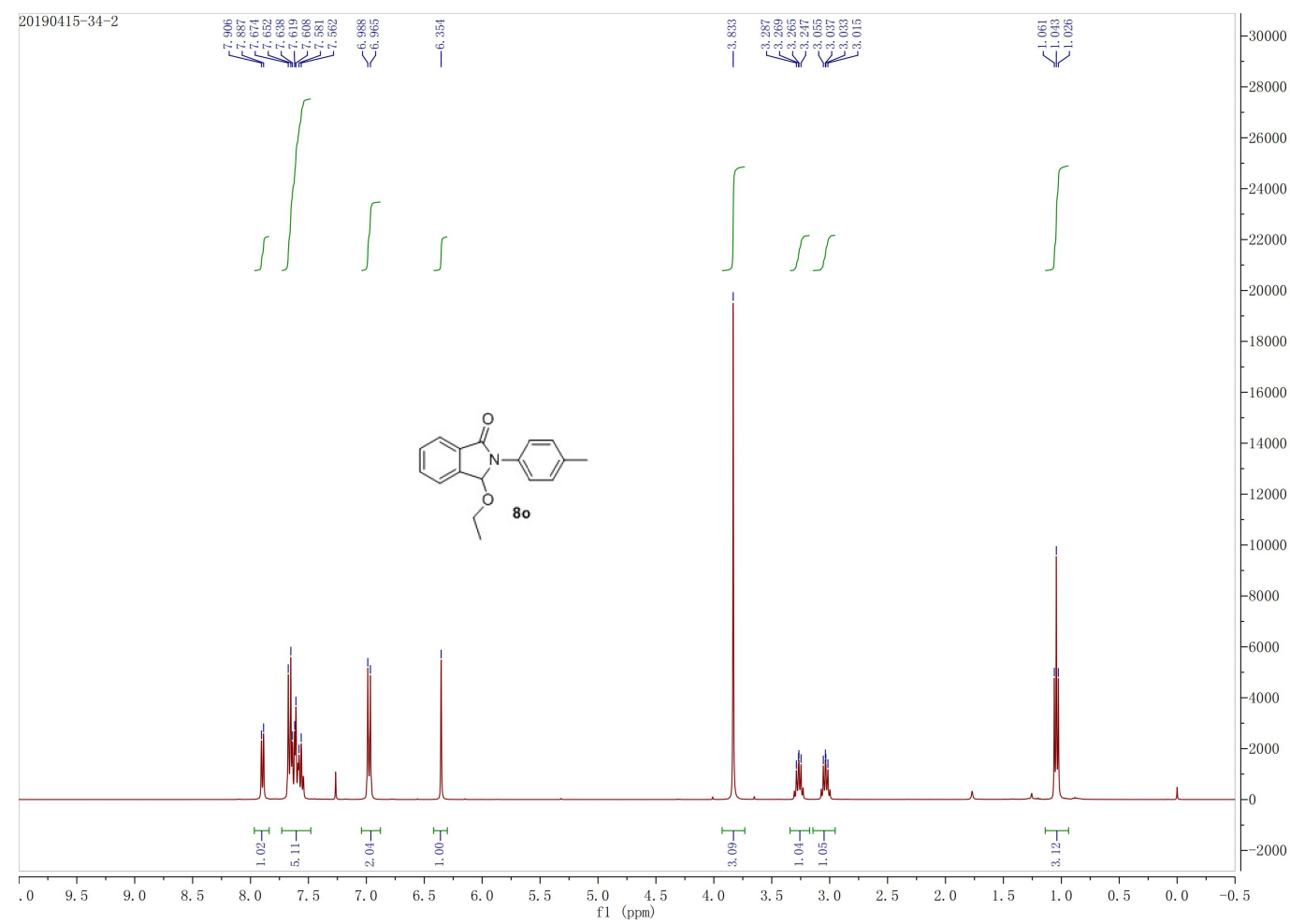

Figure S79. ${ }^{1} \mathrm{HNMR}\left(400 \mathrm{MHz}, \mathrm{CDCl}_{3}\right)$ of compound 80

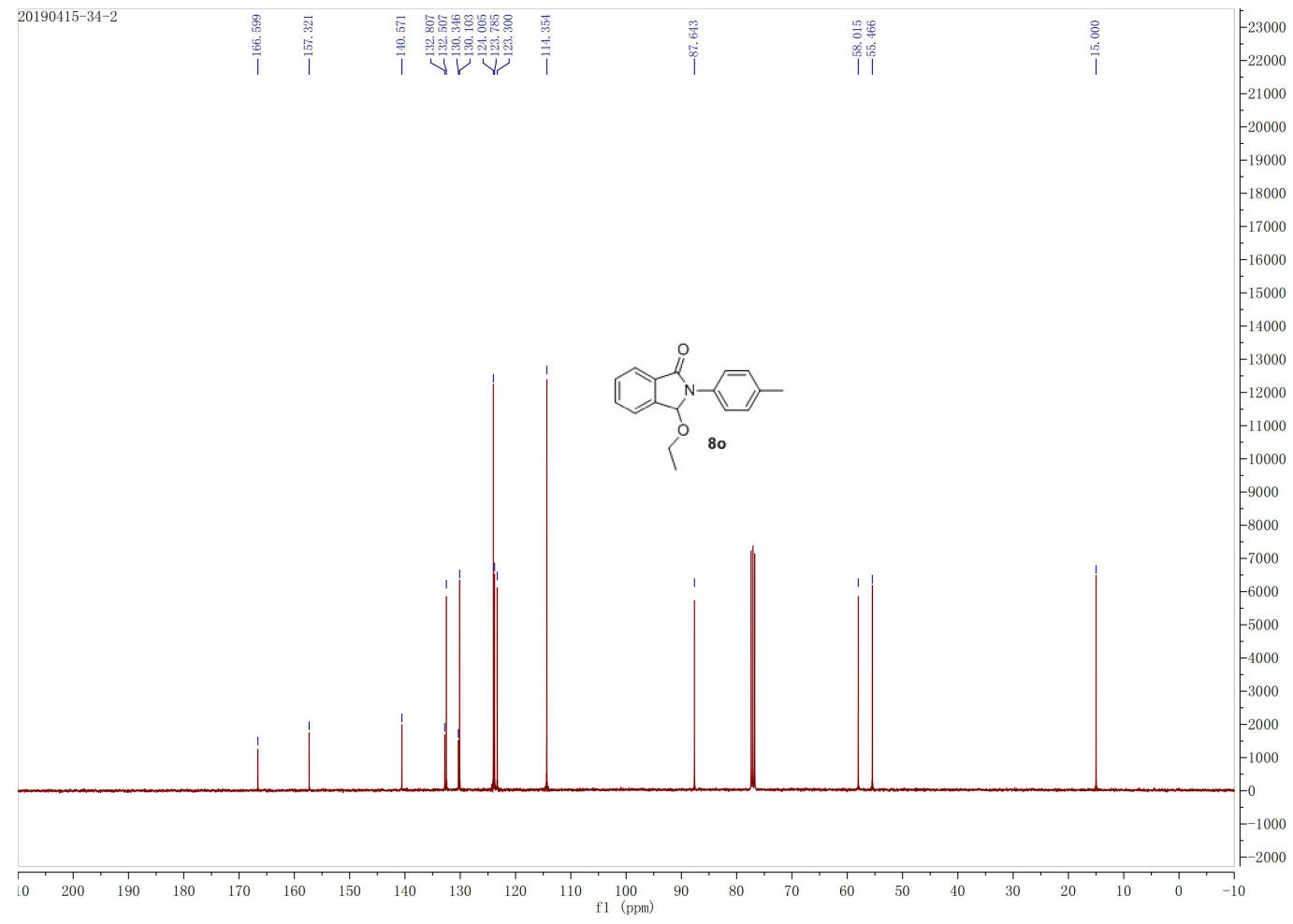

Figure S80. ${ }^{13} \mathrm{CNMR}\left(100 \mathrm{MHz}, \mathrm{CDCl}_{3}\right)$ of compound 80 


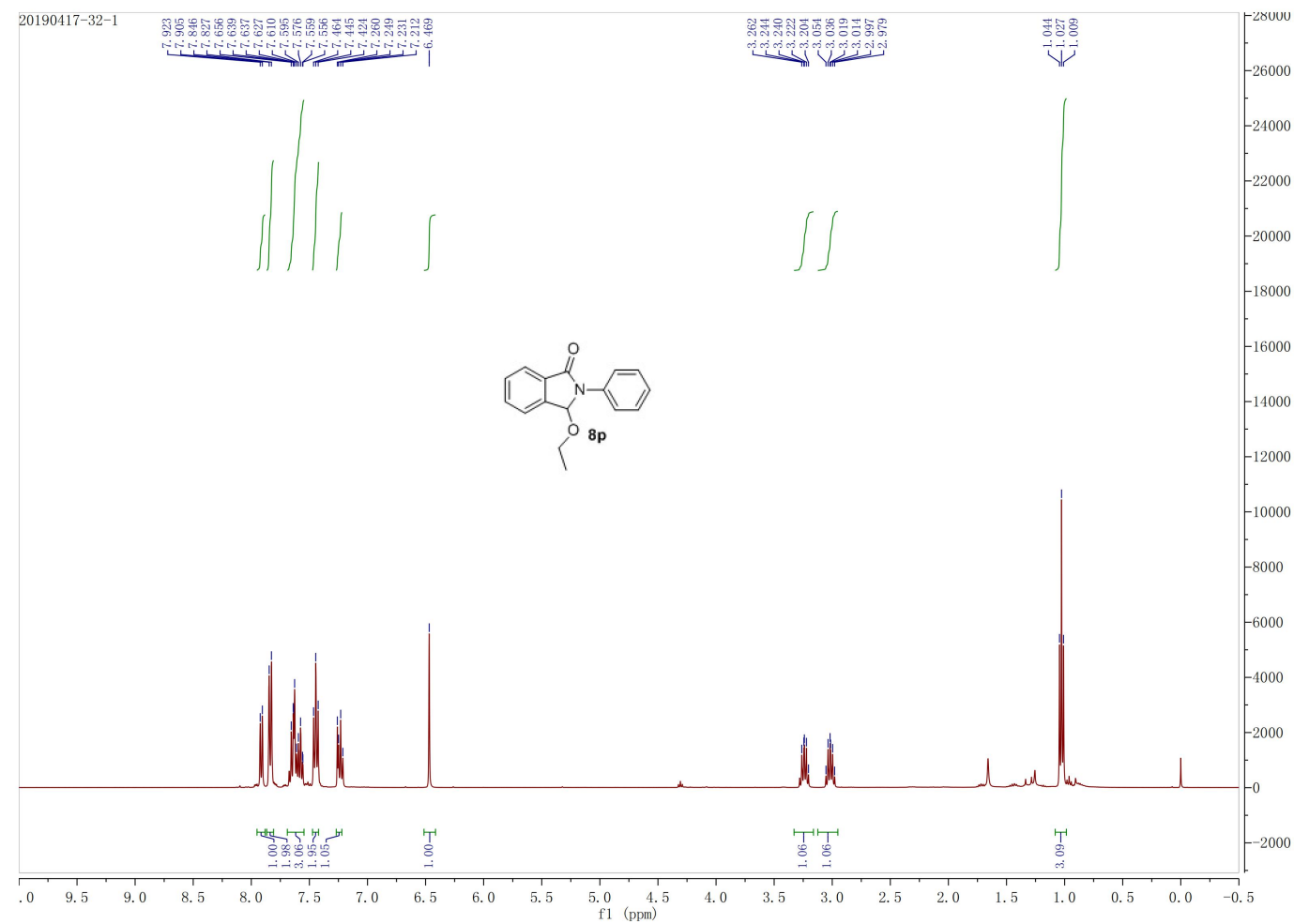

Figure S81. ${ }^{1} \mathrm{HNMR}\left(400 \mathrm{MHz}, \mathrm{CDCl}_{3}\right)$ of compound 8p

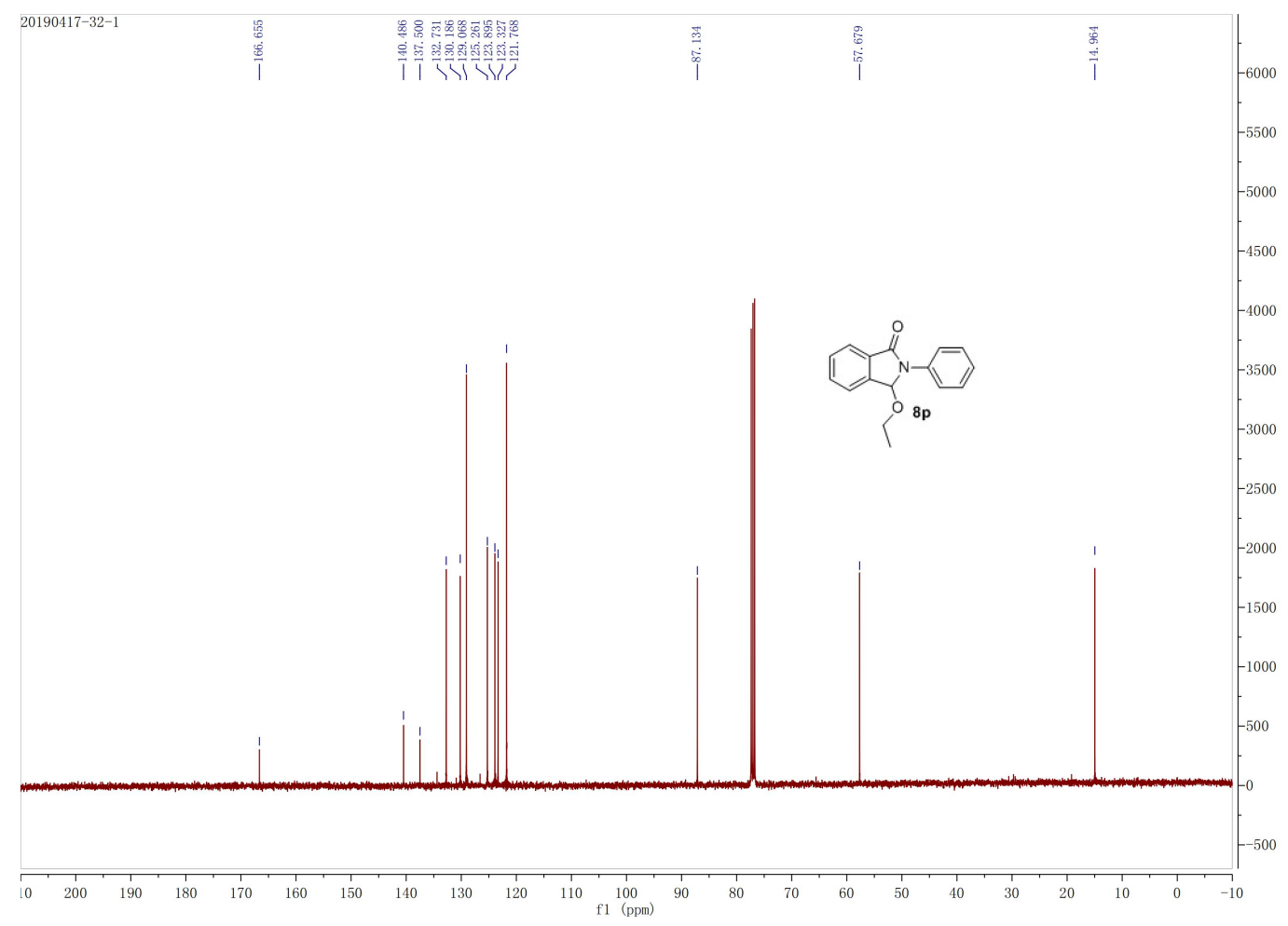

Figure S82. ${ }^{13} \mathrm{CNMR}\left(100 \mathrm{MHz}, \mathrm{CDCl}_{3}\right)$ of compound 8p 


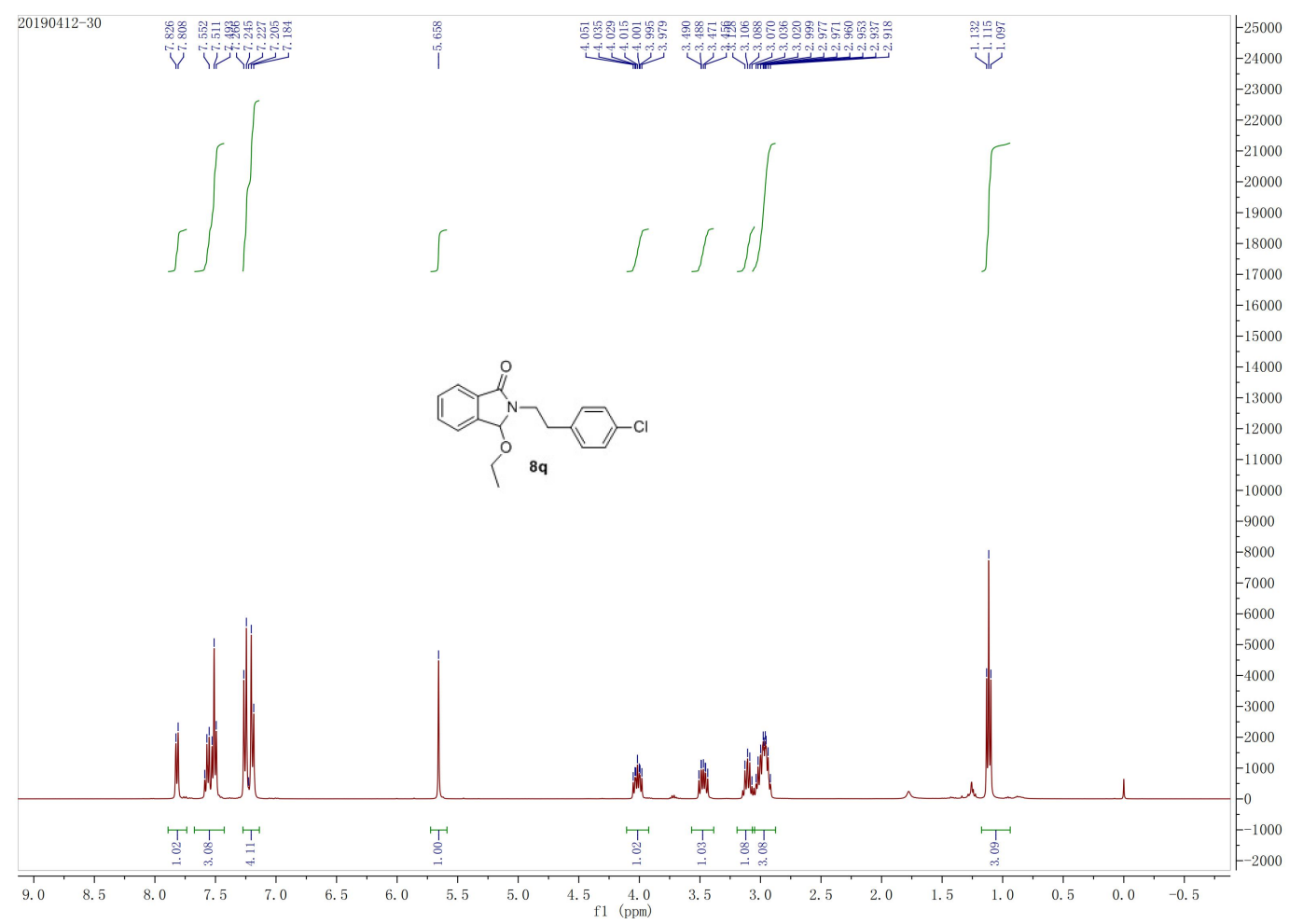

Figure S83. ${ }^{1} \mathrm{HNMR}\left(400 \mathrm{MHz}, \mathrm{CDCl}_{3}\right)$ of compound 8q

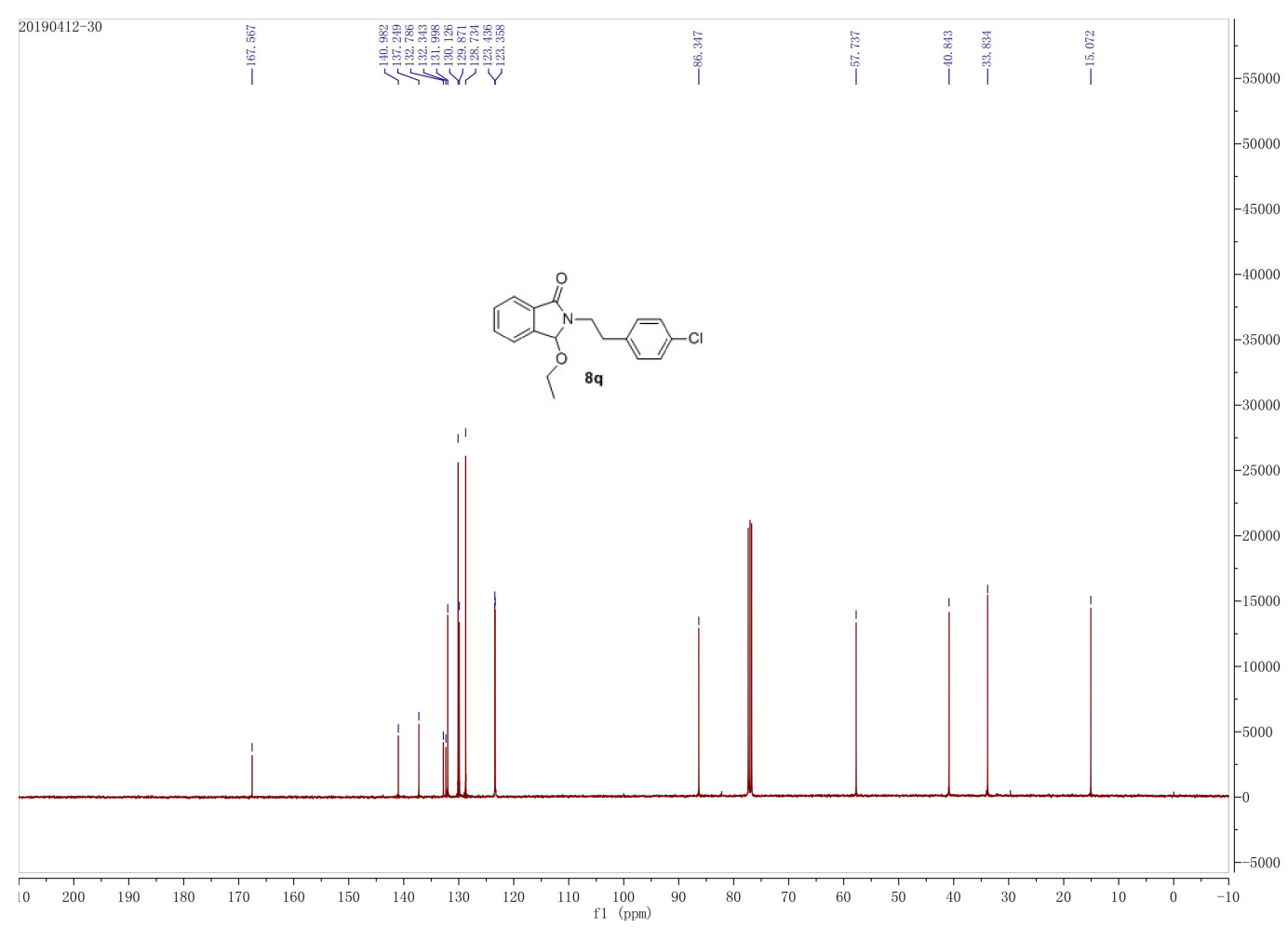

Figure S84. ${ }^{13} \mathrm{CNMR}\left(100 \mathrm{MHz}, \mathrm{CDCl}_{3}\right)$ of compound $8 \mathrm{q}$ 


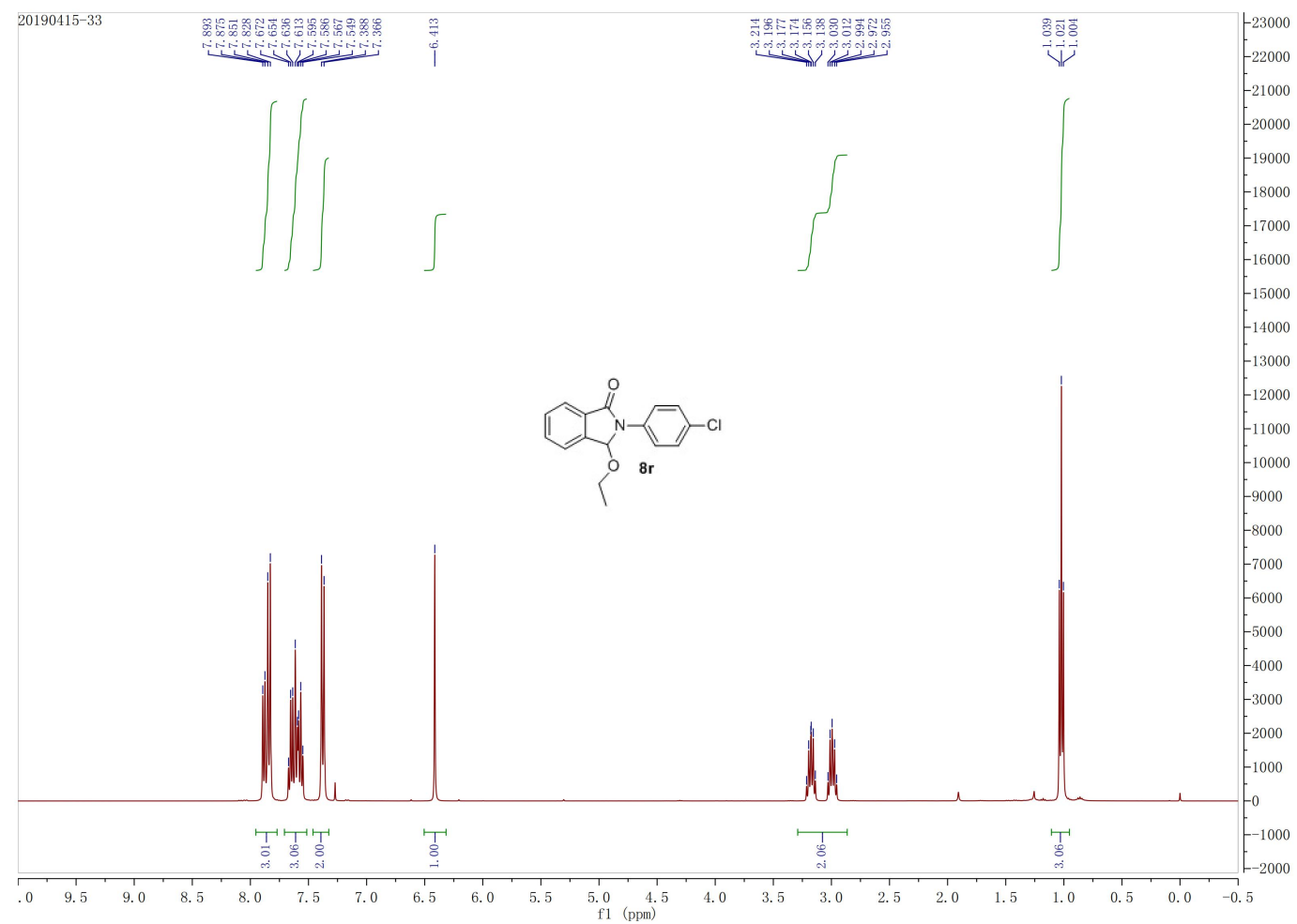

Figure S85. ${ }^{1} \mathrm{HNMR}\left(400 \mathrm{MHz}, \mathrm{CDCl}_{3}\right)$ of compound $8 \mathrm{r}$

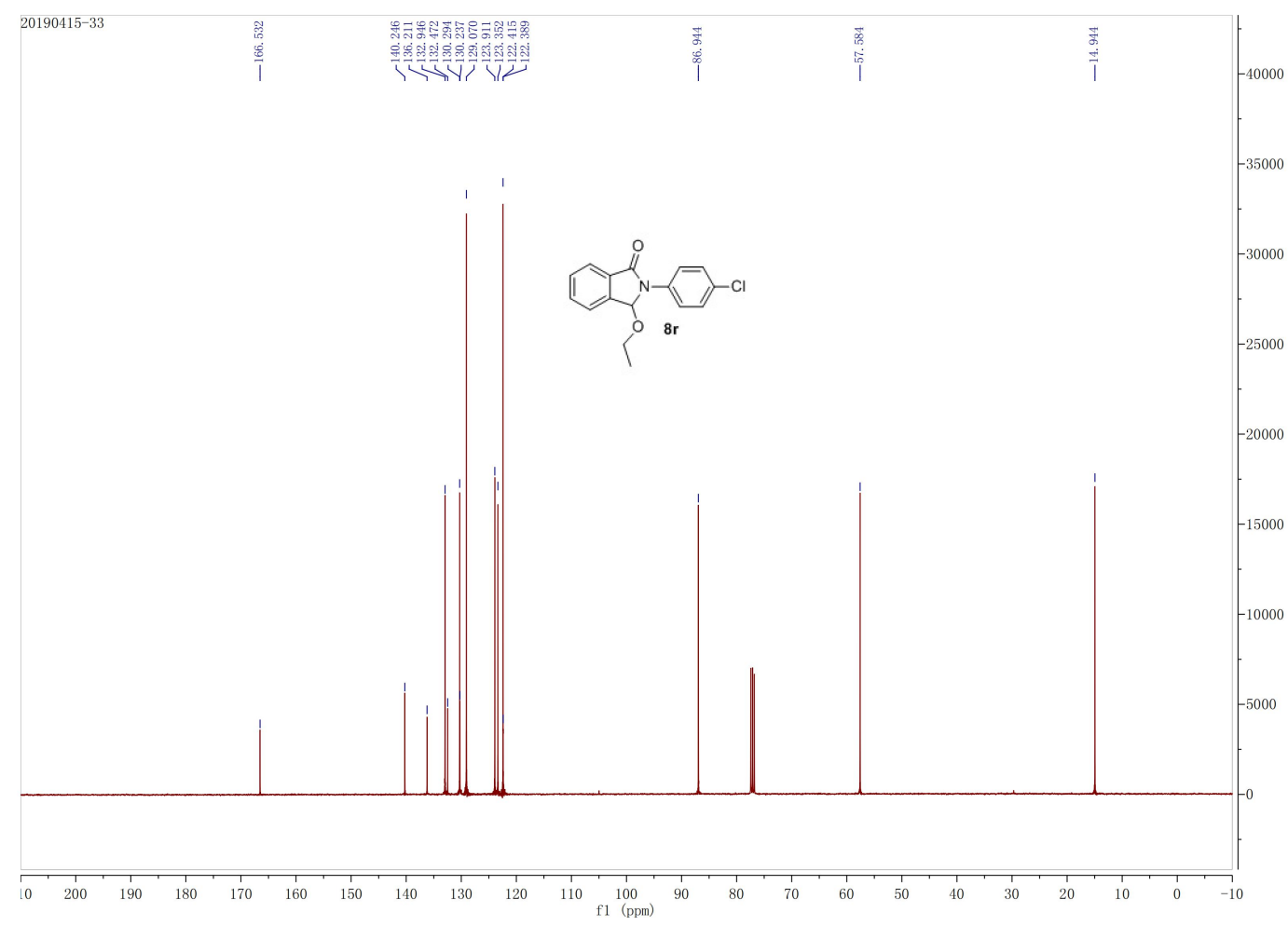

Figure S86. ${ }^{13} \mathrm{CNMR}\left(100 \mathrm{MHz}, \mathrm{CDCl}_{3}\right)$ of compound $8 \mathrm{r}$ 


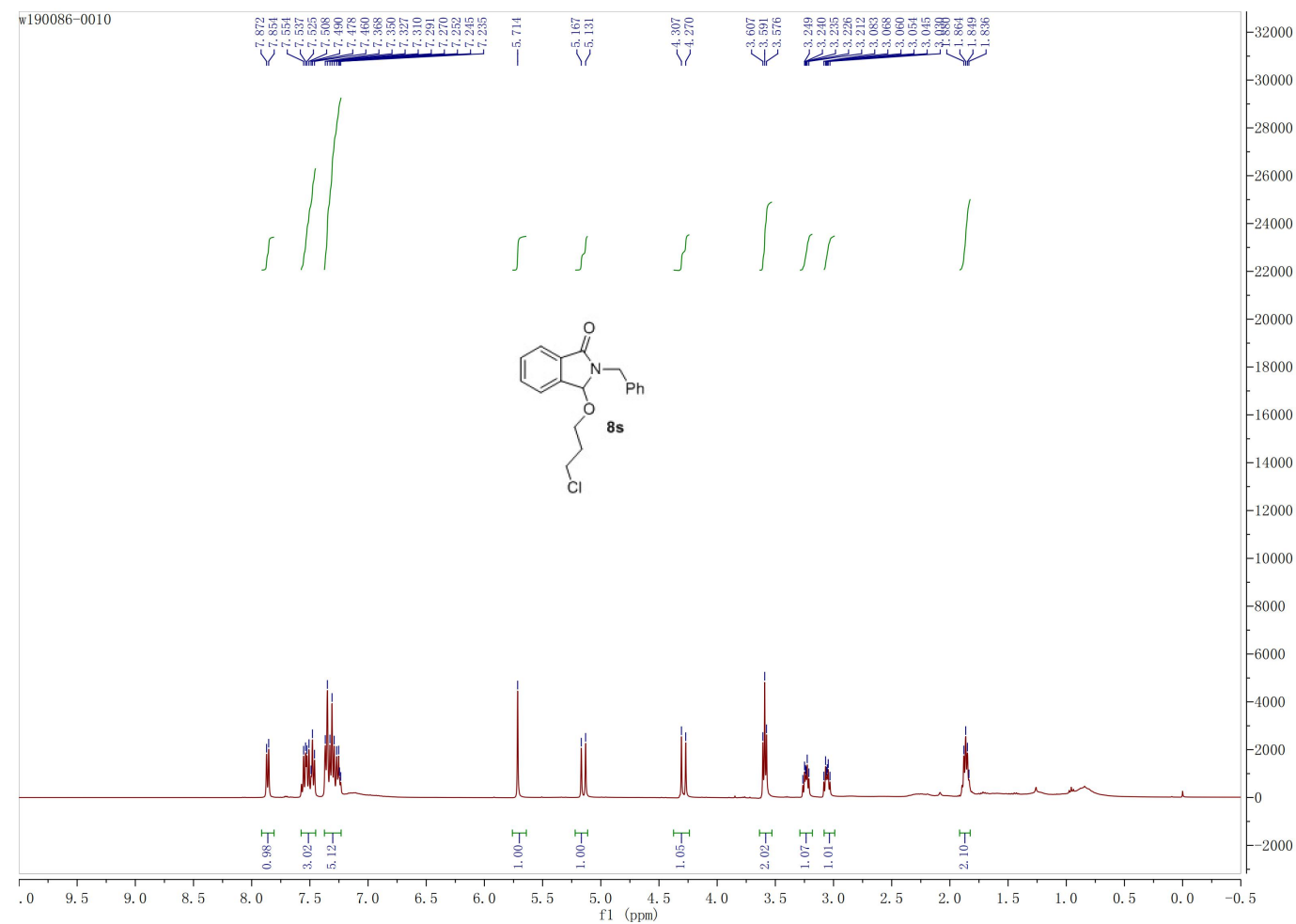

Figure S87. ${ }^{1} \mathrm{HNMR}\left(400 \mathrm{MHz}, \mathrm{CDCl}_{3}\right)$ of compound 8s

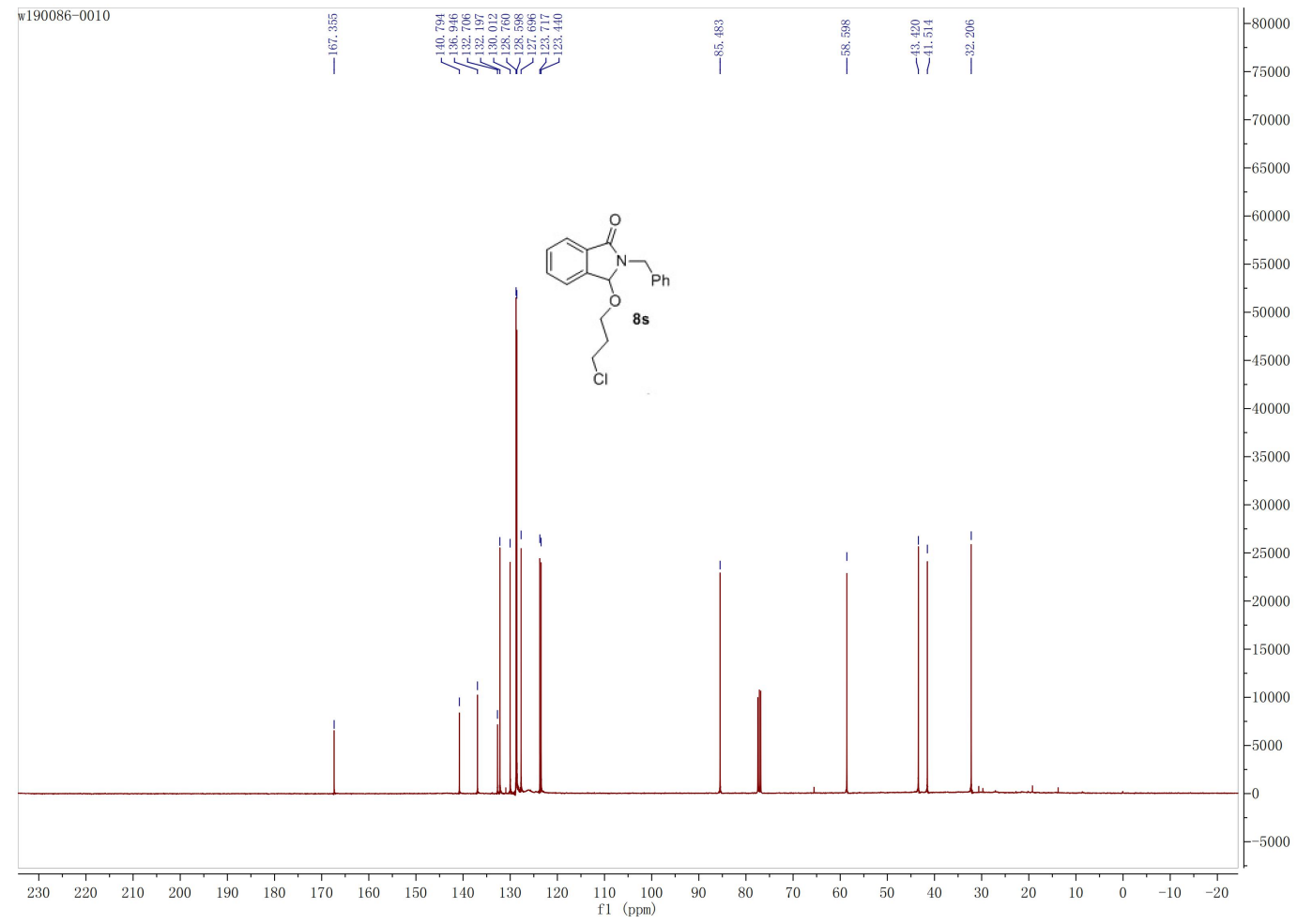

Figure S88. ${ }^{13} \mathrm{CNMR}\left(100 \mathrm{MHz}, \mathrm{CDCl}_{3}\right)$ of compound $8 \mathrm{~s}$ 


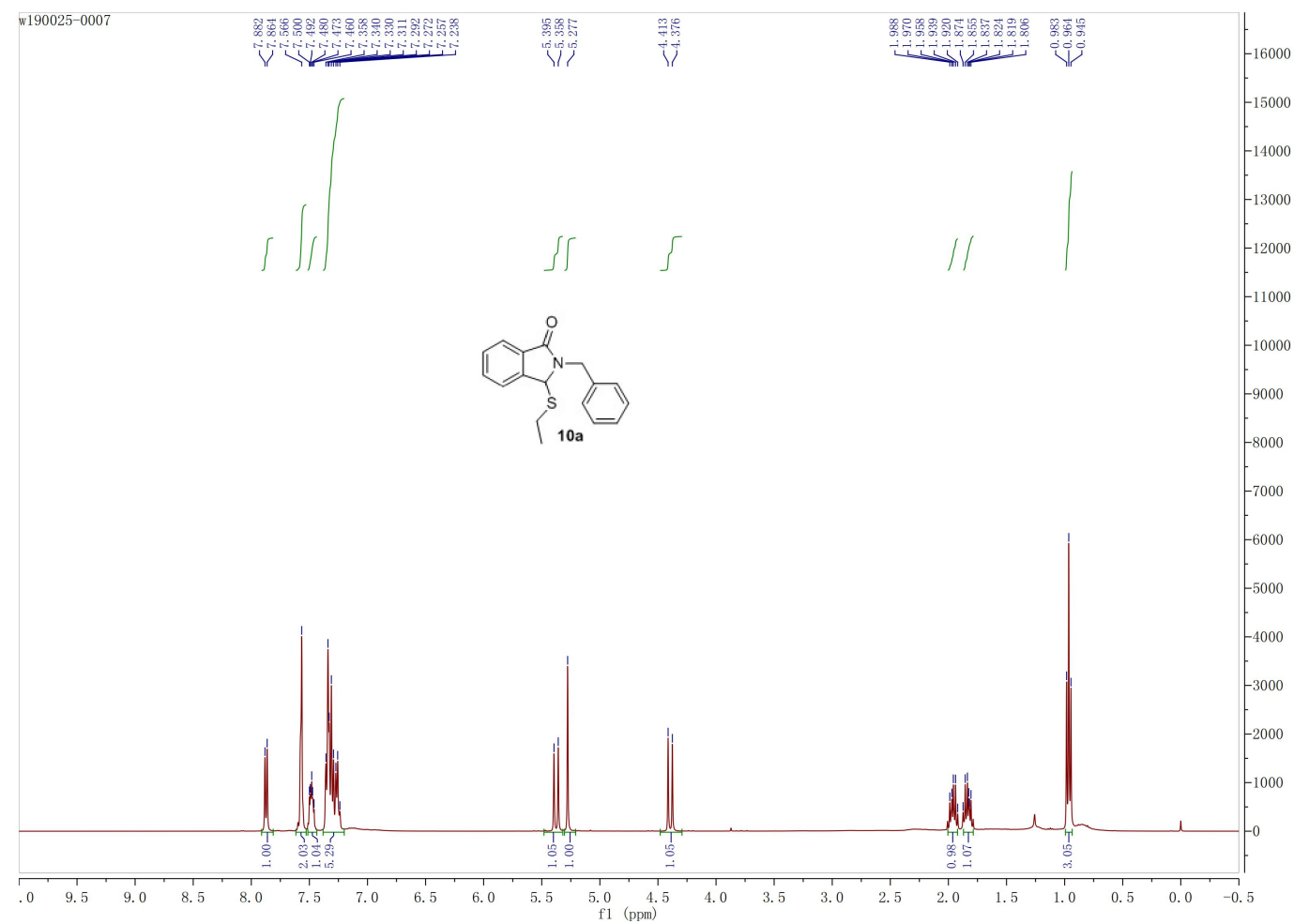

Figure S89. ${ }^{1} \mathrm{HNMR}\left(400 \mathrm{MHz}, \mathrm{CDCl}_{3}\right)$ of compound $10 \mathrm{a}$

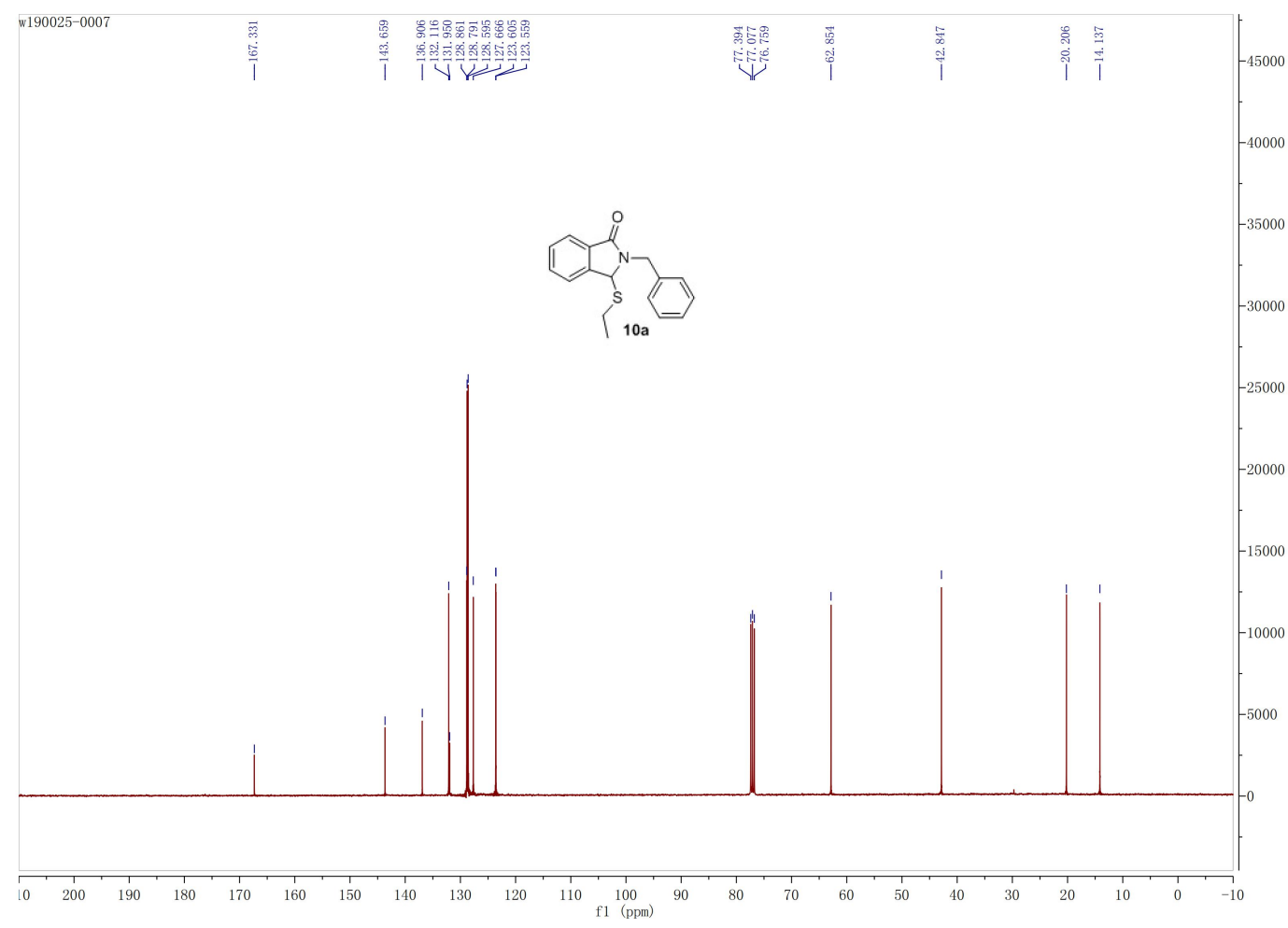

Figure S90. ${ }^{13} \mathrm{CNMR}\left(100 \mathrm{MHz}, \mathrm{CDCl}_{3}\right)$ of compound $10 \mathrm{a}$ 


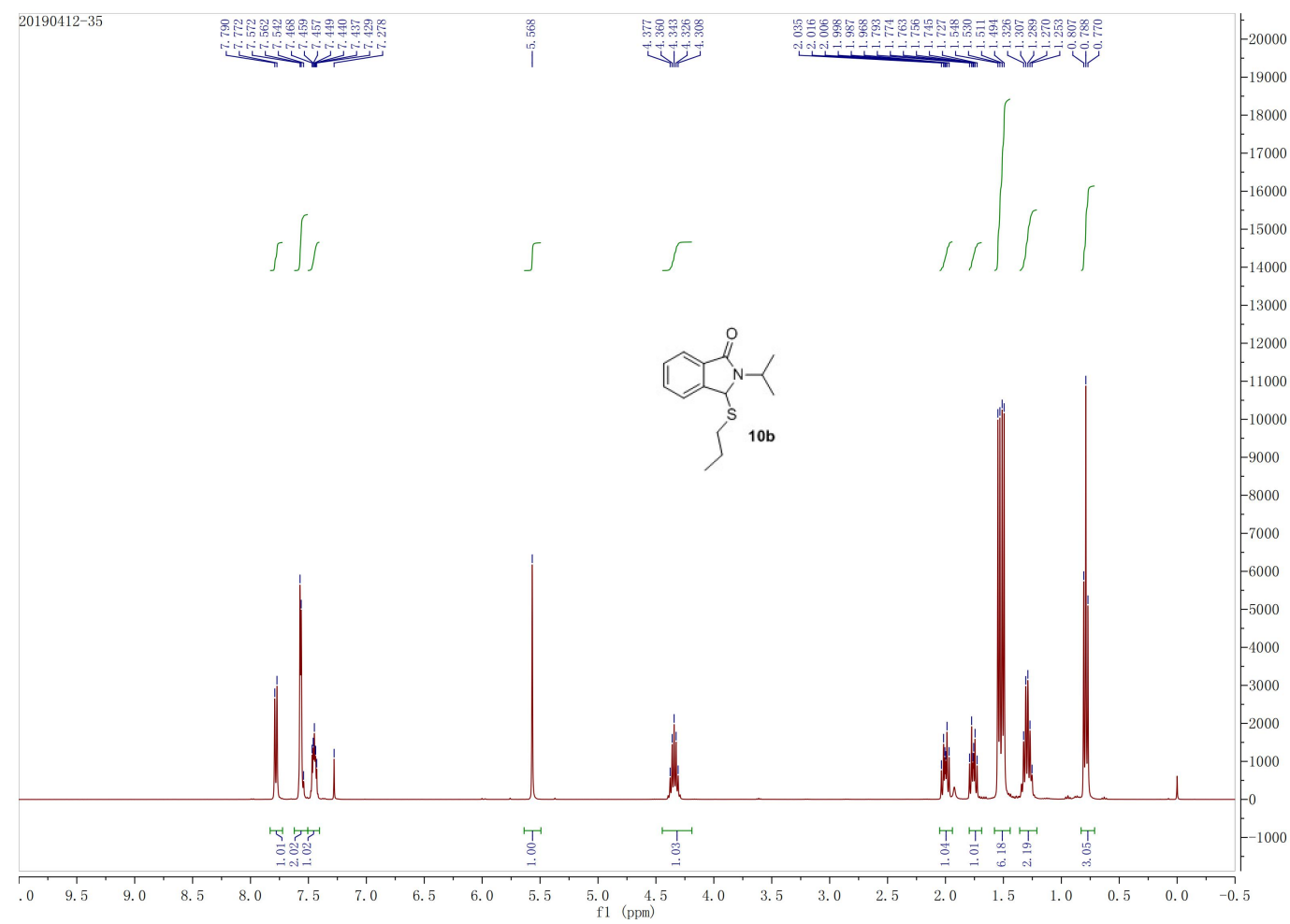

Figure S91. ${ }^{1} \mathrm{HNMR}\left(400 \mathrm{MHz}, \mathrm{CDCl}_{3}\right)$ of compound $10 \mathrm{~b}$

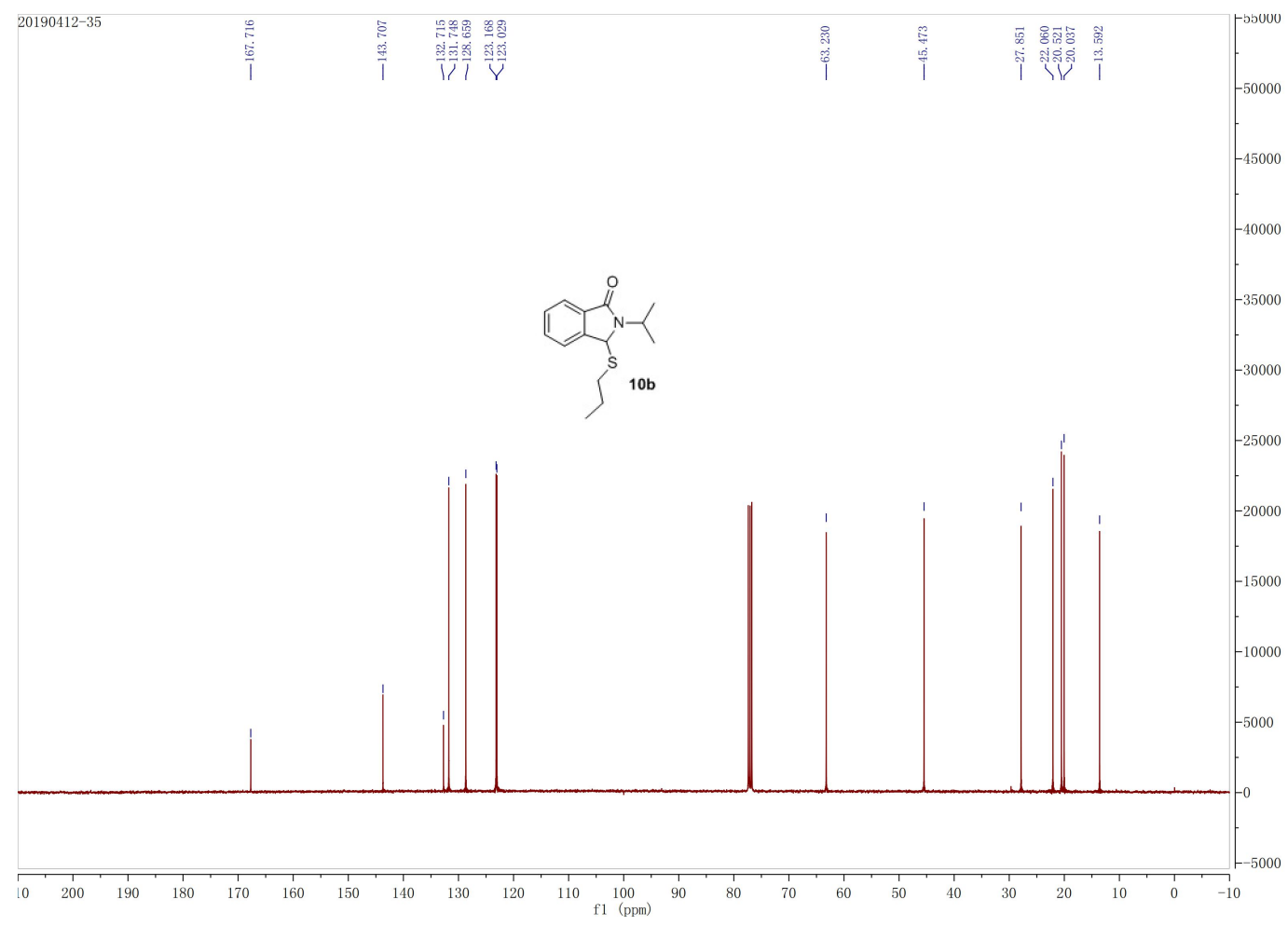

Figure S92. ${ }^{13} \mathrm{CNMR}\left(100 \mathrm{MHz}, \mathrm{CDCl}_{3}\right)$ of compound $10 \mathrm{~b}$ 


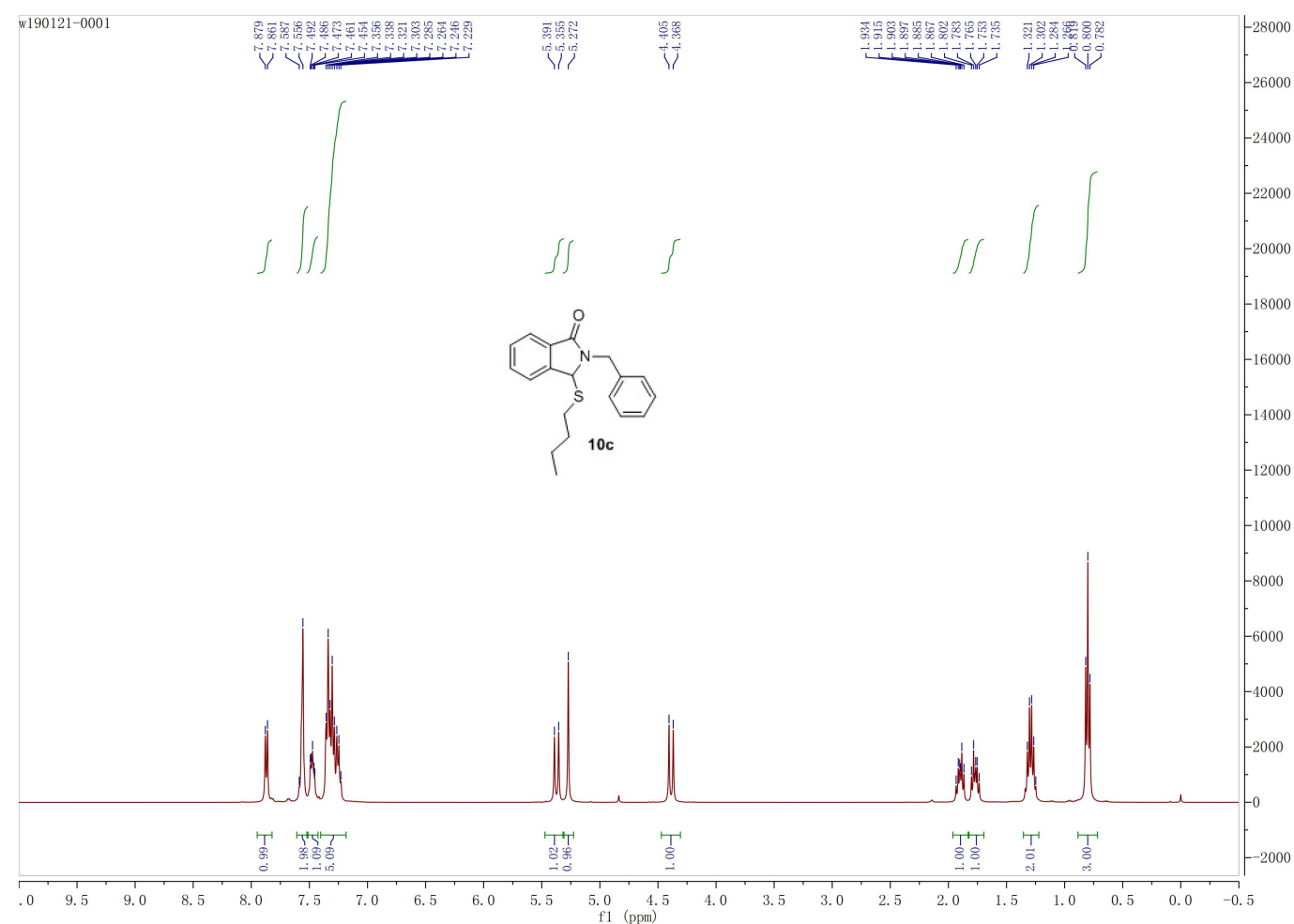

Figure S93. ${ }^{1} \mathrm{HNMR}\left(400 \mathrm{MHz}, \mathrm{CDCl}_{3}\right)$ of compound $10 \mathrm{c}$

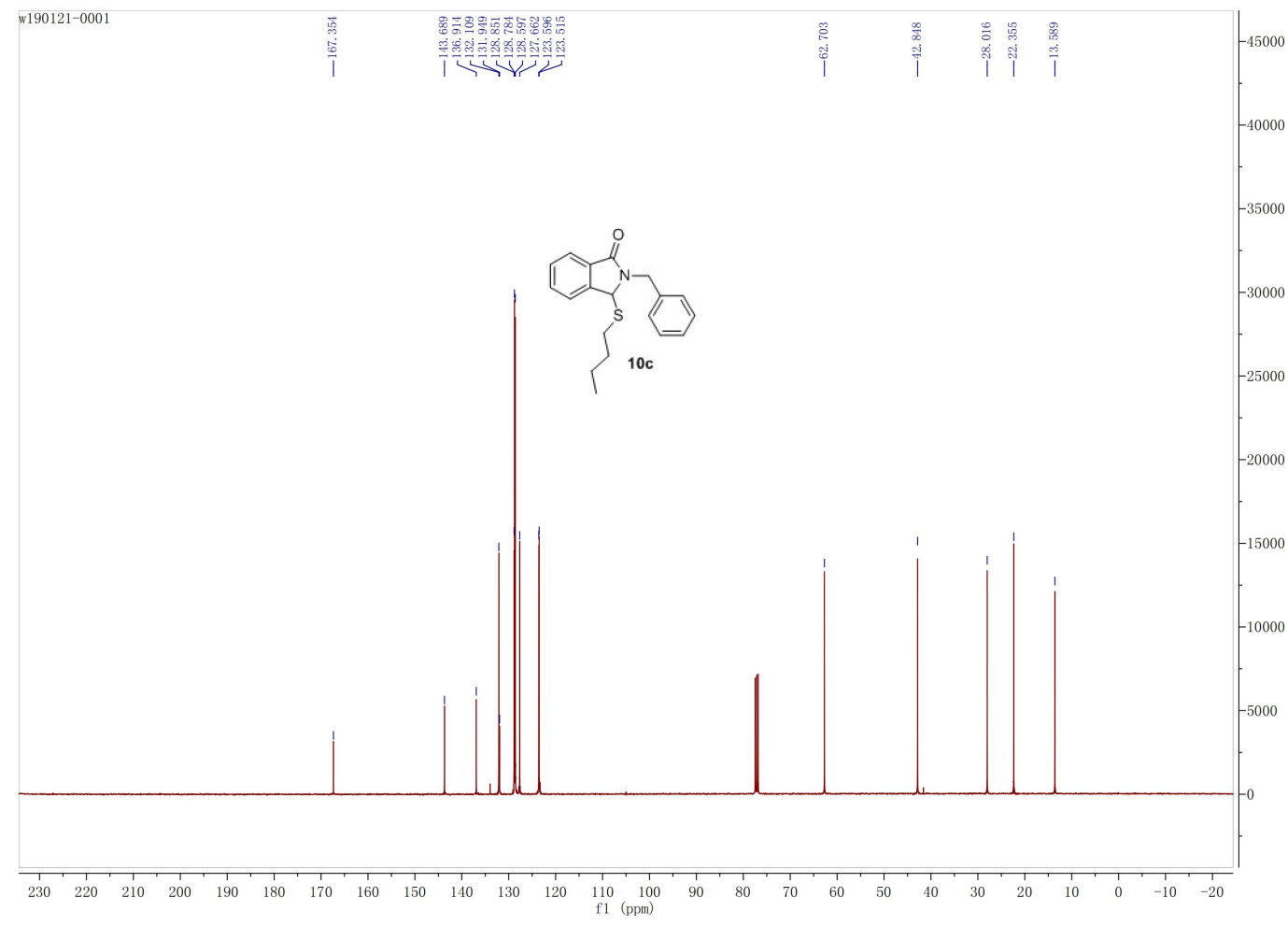

Figure S94. ${ }^{13} \mathrm{CNMR}\left(100 \mathrm{MHz}, \mathrm{CDCl}_{3}\right)$ of compound $10 \mathrm{c}$ 


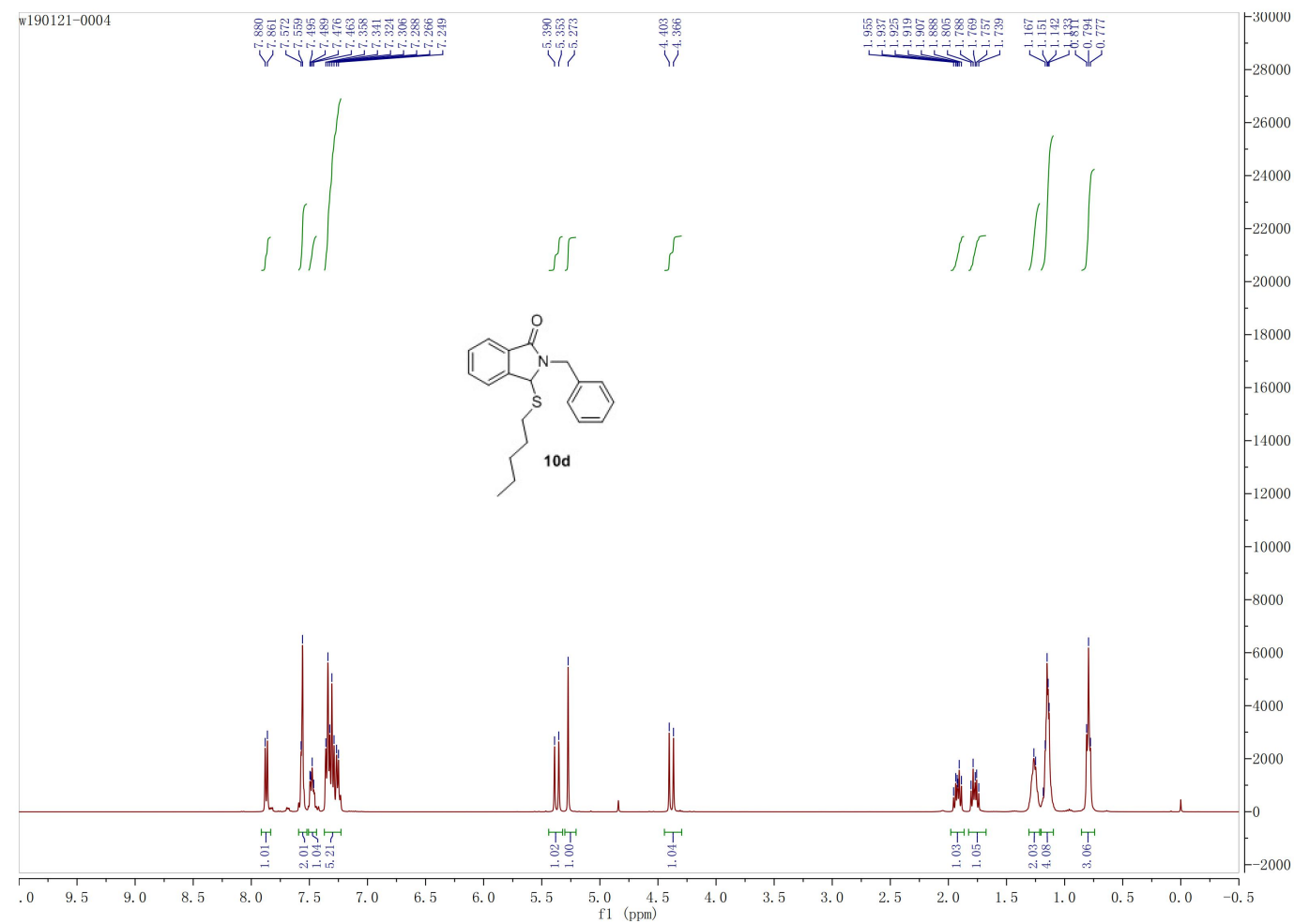

Figure S95. ${ }^{1} \mathrm{HNMR}\left(400 \mathrm{MHz}, \mathrm{CDCl}_{3}\right)$ of compound 10d

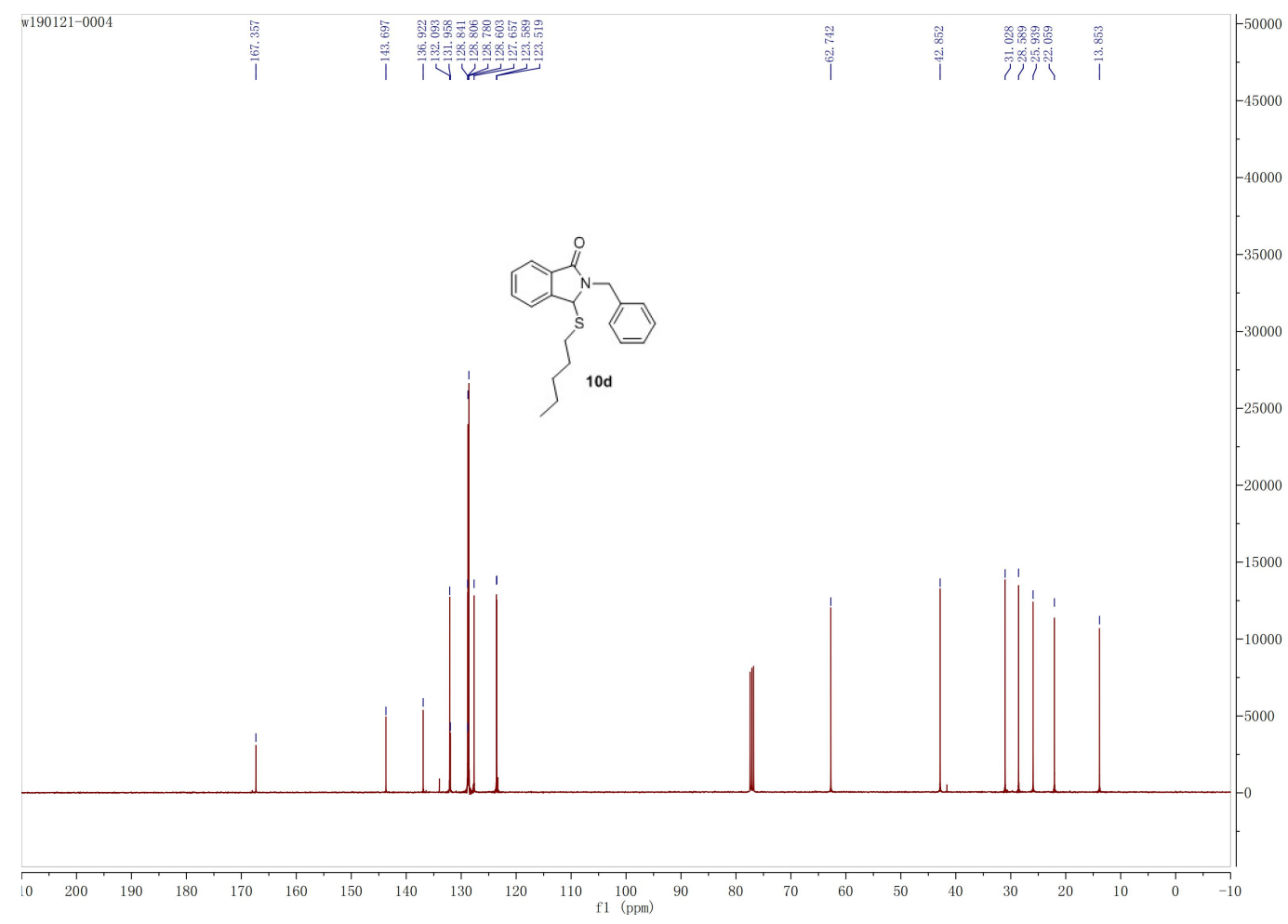

Figure S96. ${ }^{13} \mathrm{CNMR}\left(100 \mathrm{MHz}, \mathrm{CDCl}_{3}\right)$ of compound $10 \mathrm{~d}$ 


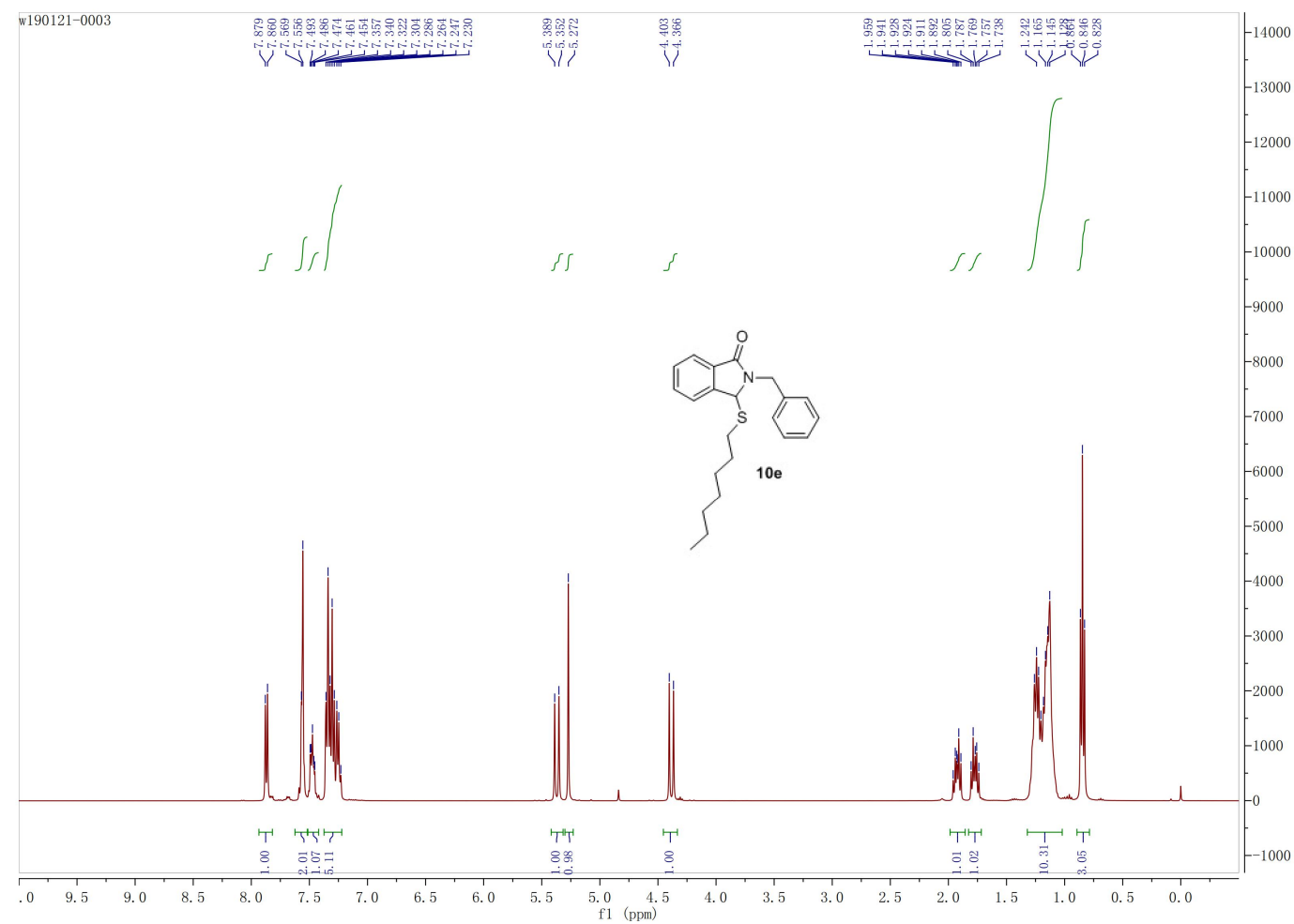

Figure S97. ${ }^{1} \mathrm{HNMR}\left(400 \mathrm{MHz}, \mathrm{CDCl}_{3}\right)$ of compound $10 \mathrm{e}$

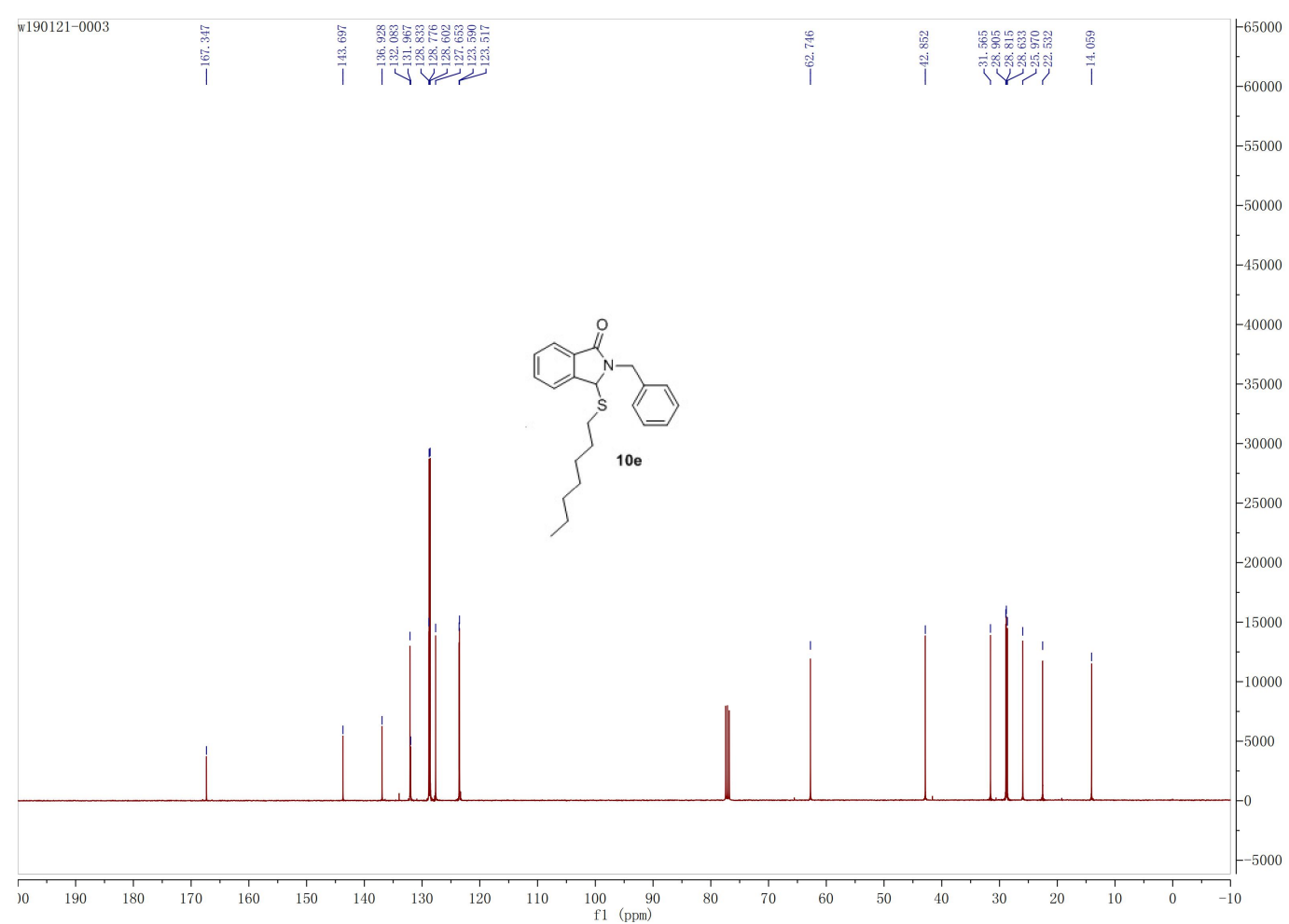

Figure S98. ${ }^{13} \mathrm{CNMR}\left(100 \mathrm{MHz}, \mathrm{CDCl}_{3}\right)$ of compound $10 \mathrm{e}$ 


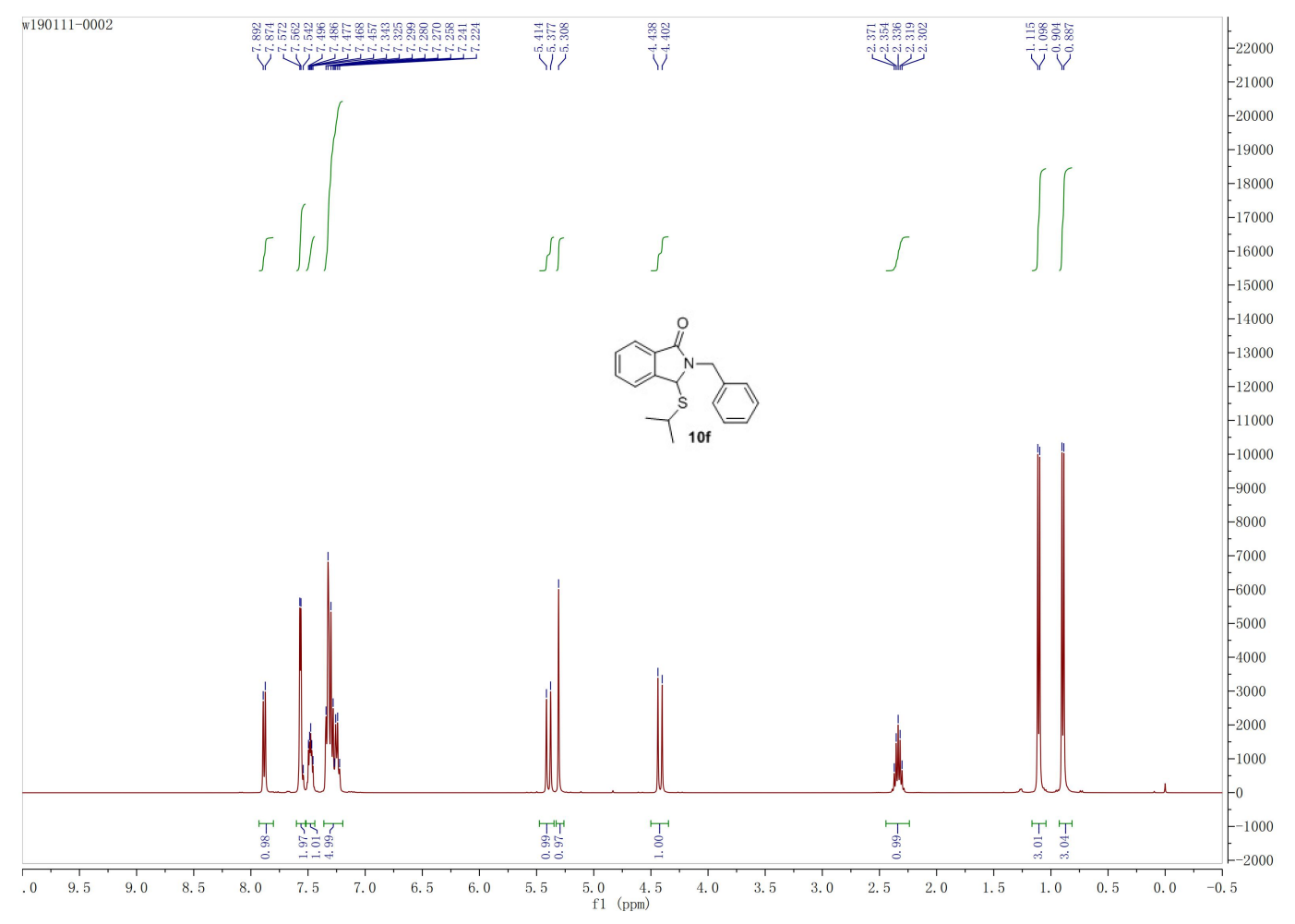

Figure S99. ${ }^{1} \mathrm{HNMR}\left(400 \mathrm{MHz}, \mathrm{CDCl}_{3}\right)$ of compound $10 \mathrm{f}$

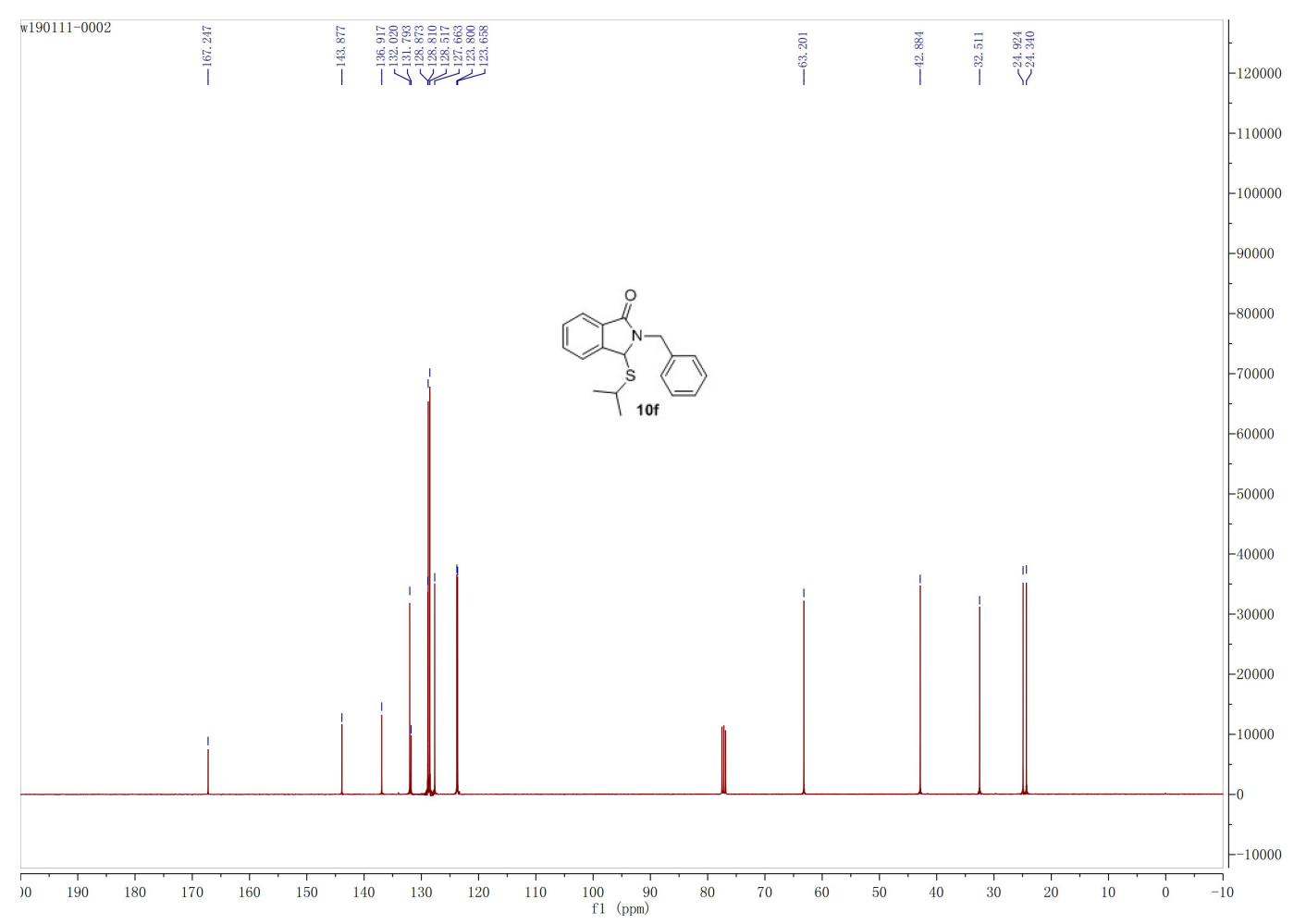

Figure S100. ${ }^{13} \mathrm{CNMR}\left(100 \mathrm{MHz}, \mathrm{CDCl}_{3}\right)$ of compound $10 \mathrm{f}$ 


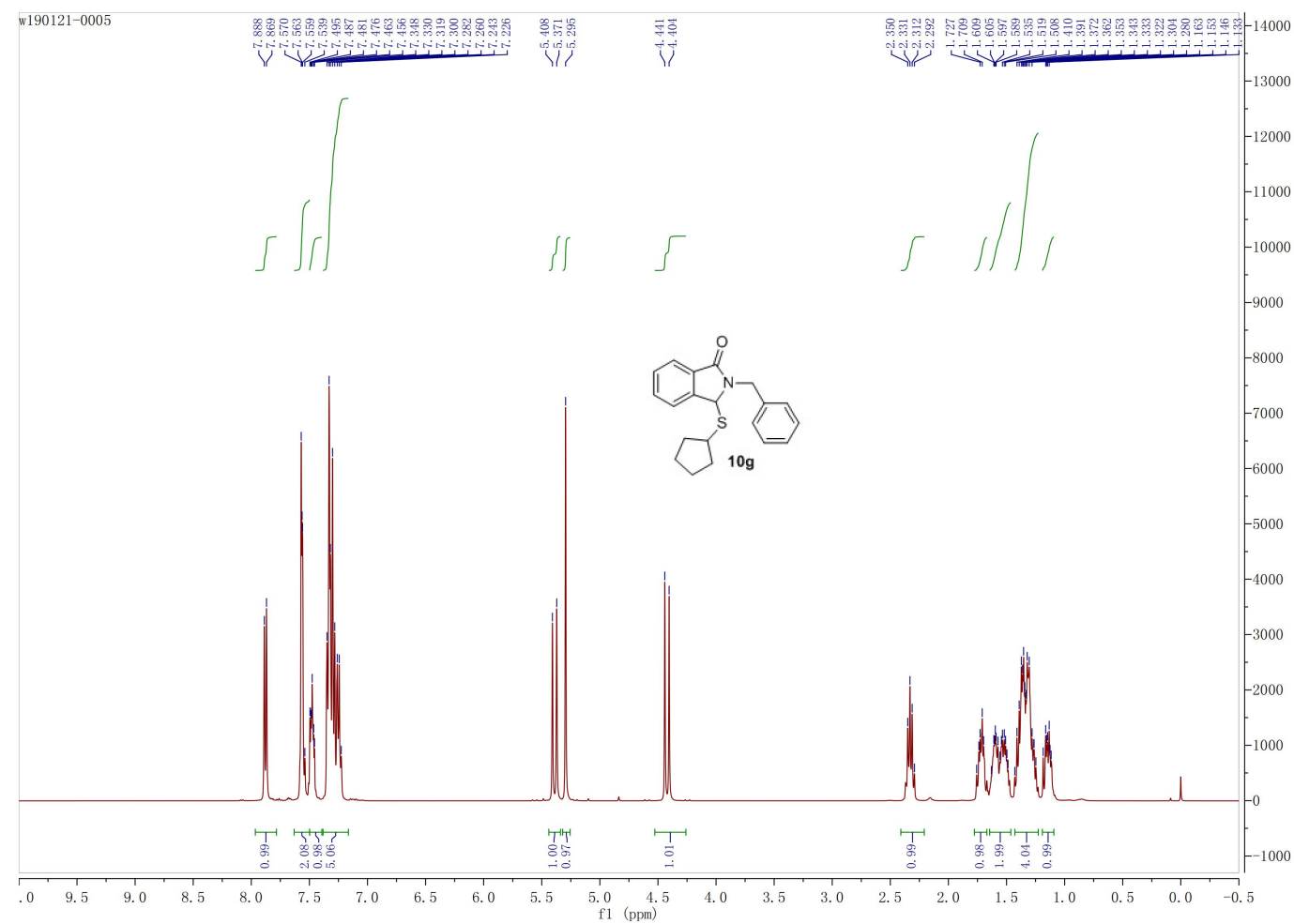

Figure S101. ${ }^{1} \mathrm{HNMR}\left(400 \mathrm{MHz}, \mathrm{CDCl}_{3}\right)$ of compound $10 \mathrm{~g}$

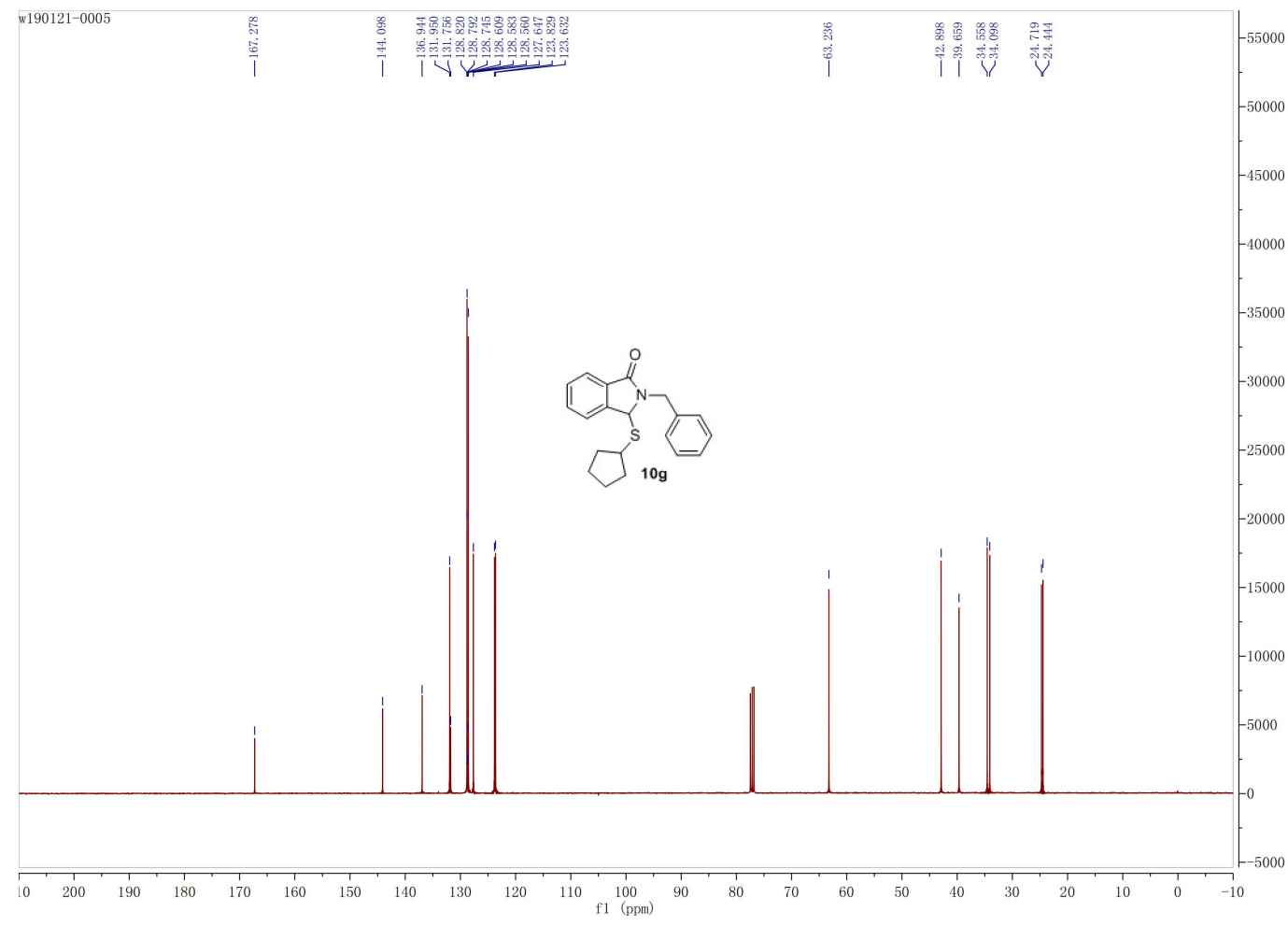

Figure S102. ${ }^{13} \mathrm{CNMR}\left(100 \mathrm{MHz}, \mathrm{CDCl}_{3}\right)$ of compound $10 \mathrm{~g}$ 


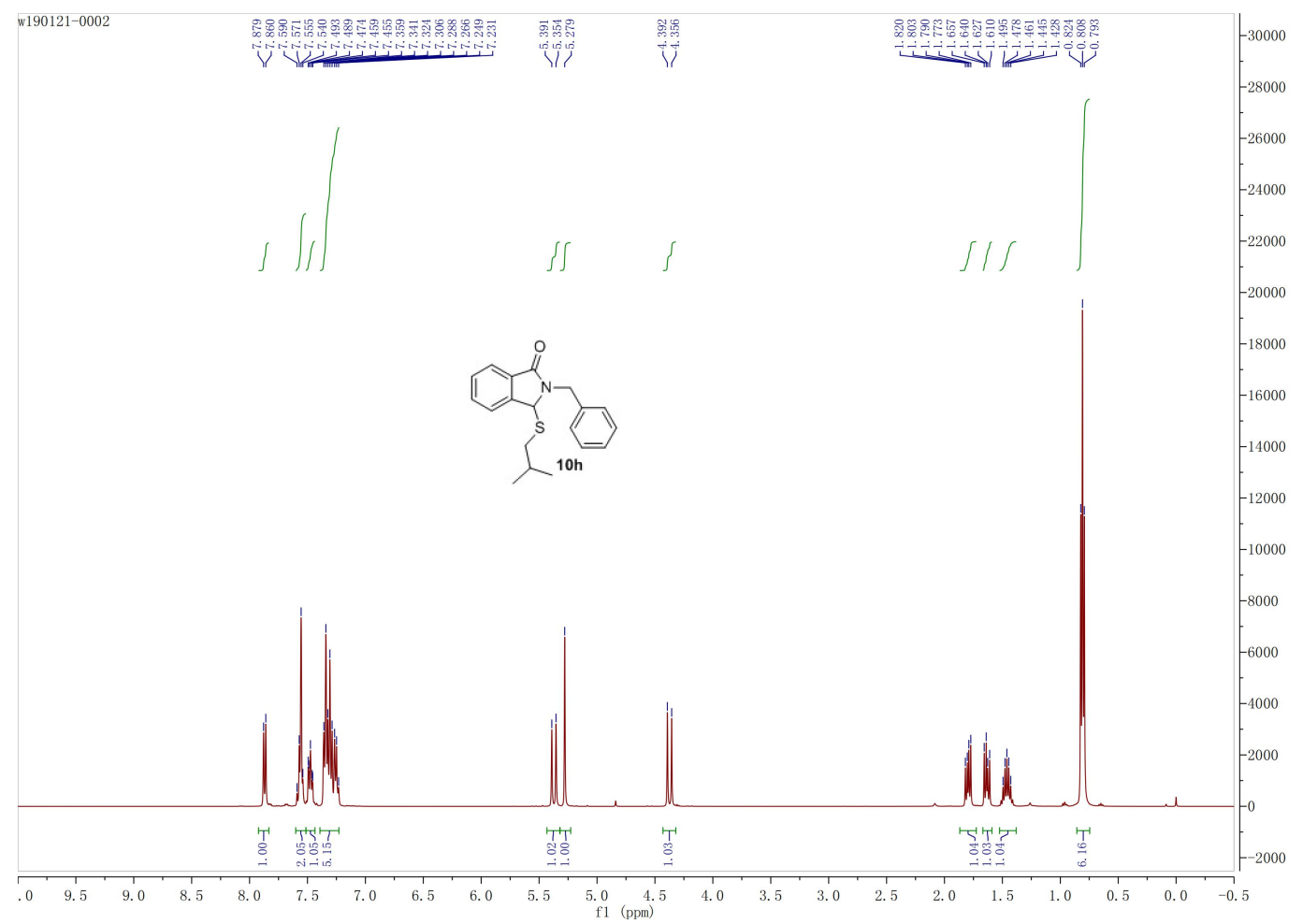

Figure S103. ${ }^{1} \mathrm{HNMR}\left(400 \mathrm{MHz}, \mathrm{CDCl}_{3}\right)$ of compound $10 \mathrm{~h}$

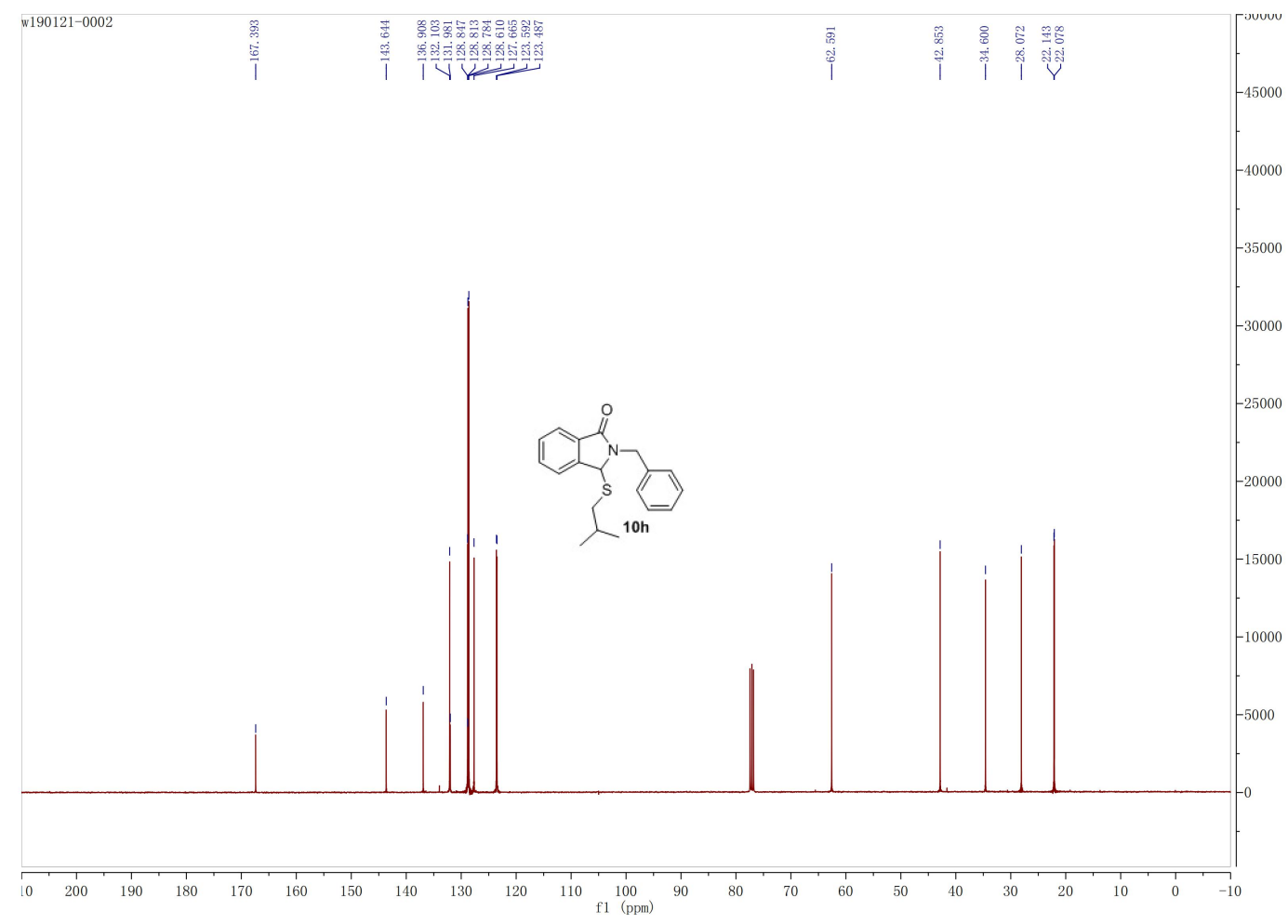

Figure S104. ${ }^{13} \mathrm{CNMR}\left(100 \mathrm{MHz}, \mathrm{CDCl}_{3}\right)$ of compound $10 \mathrm{~h}$ 


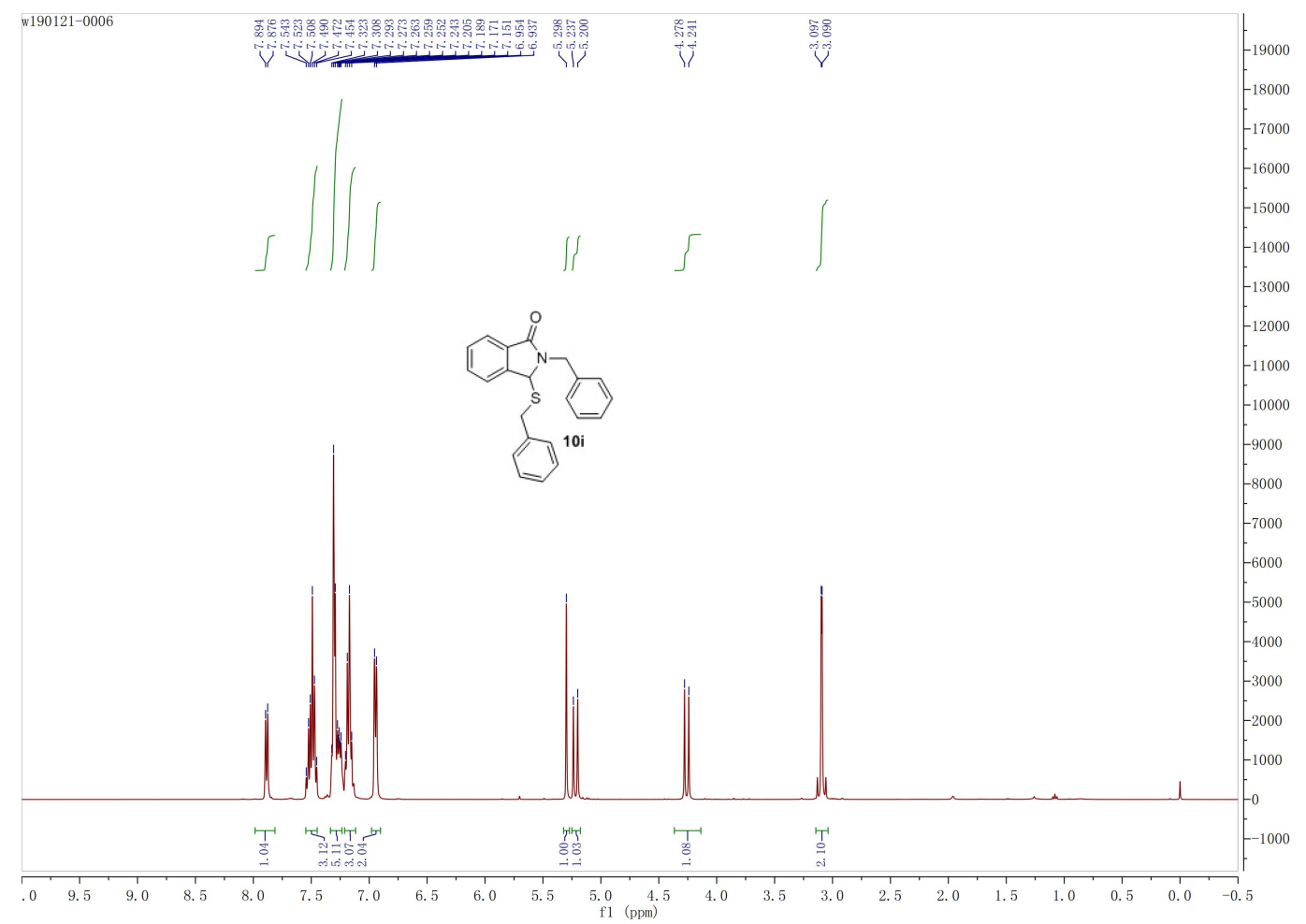

Figure S105. ${ }^{1} \mathrm{HNMR}\left(400 \mathrm{MHz}, \mathrm{CDCl}_{3}\right)$ of compound $10 \mathrm{i}$

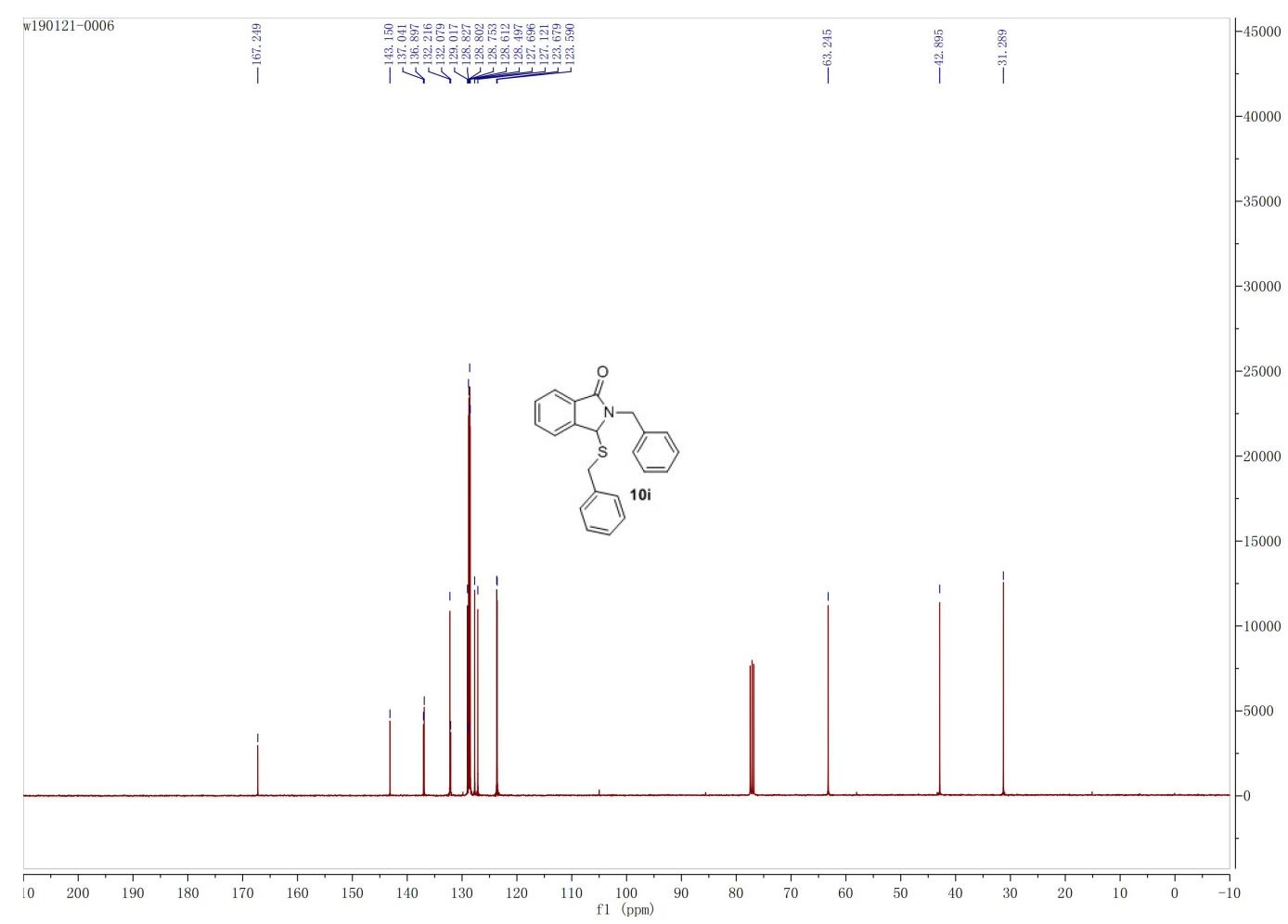

Figure S106. ${ }^{13} \mathrm{CNMR}\left(100 \mathrm{MHz}, \mathrm{CDCl}_{3}\right)$ of compound $10 \mathrm{i}$ 


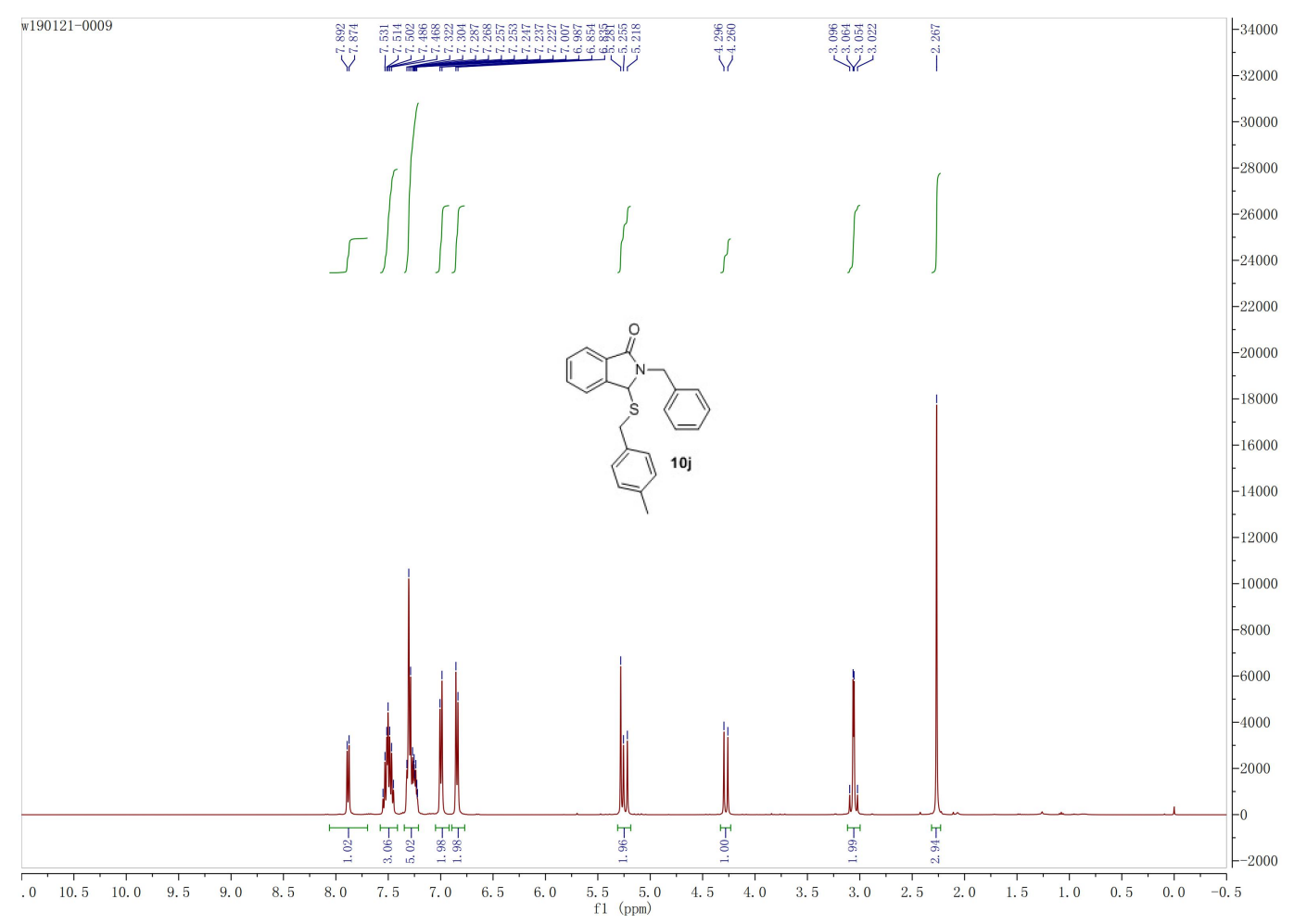

Figure S107. ${ }^{1} \mathrm{HNMR}\left(400 \mathrm{MHz}, \mathrm{CDCl}_{3}\right)$ of compound $10 \mathrm{j}$

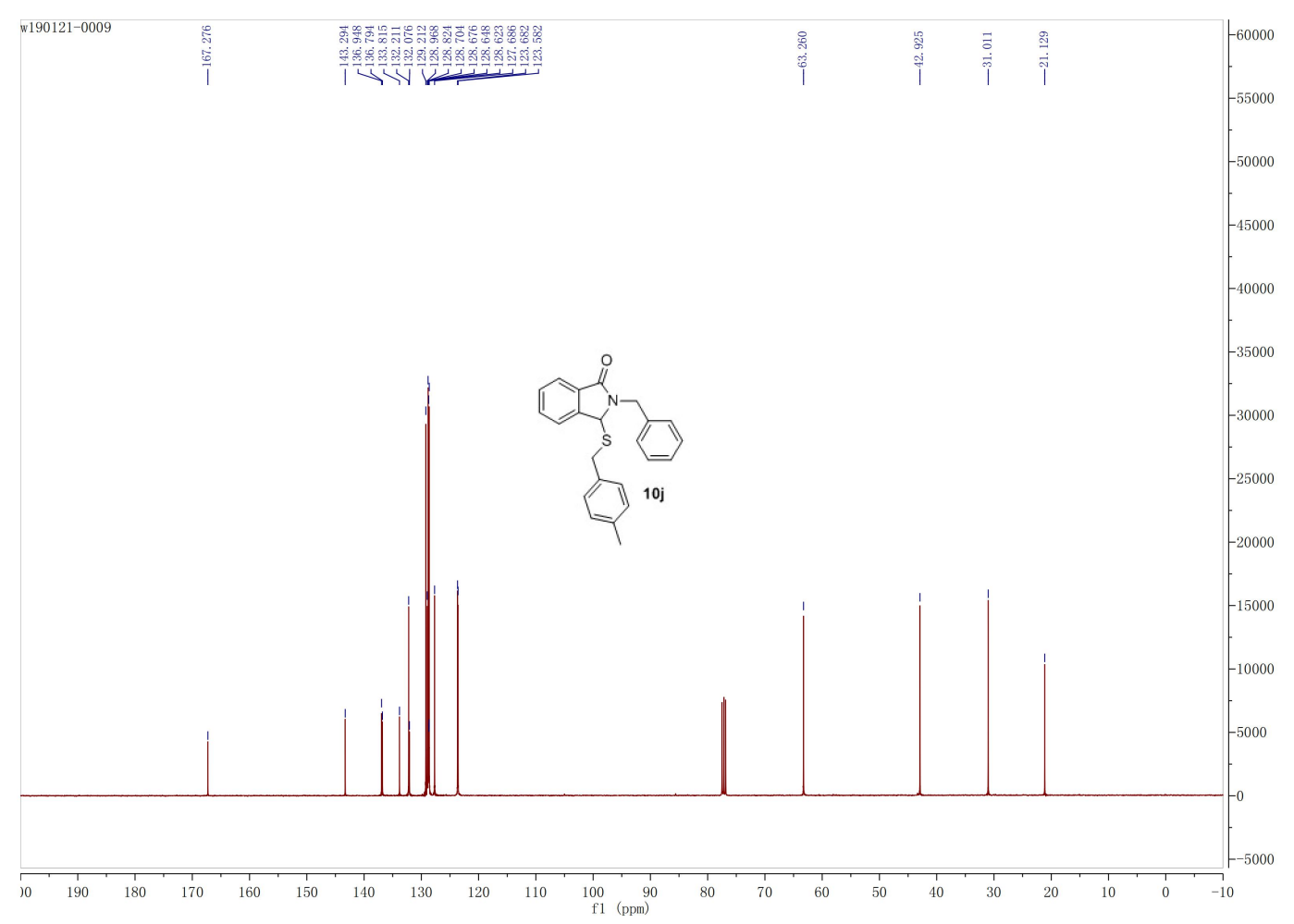

Figure S108. ${ }^{13} \mathrm{CNMR}\left(100 \mathrm{MHz}, \mathrm{CDCl}_{3}\right)$ of compound $10 \mathrm{j}$ 


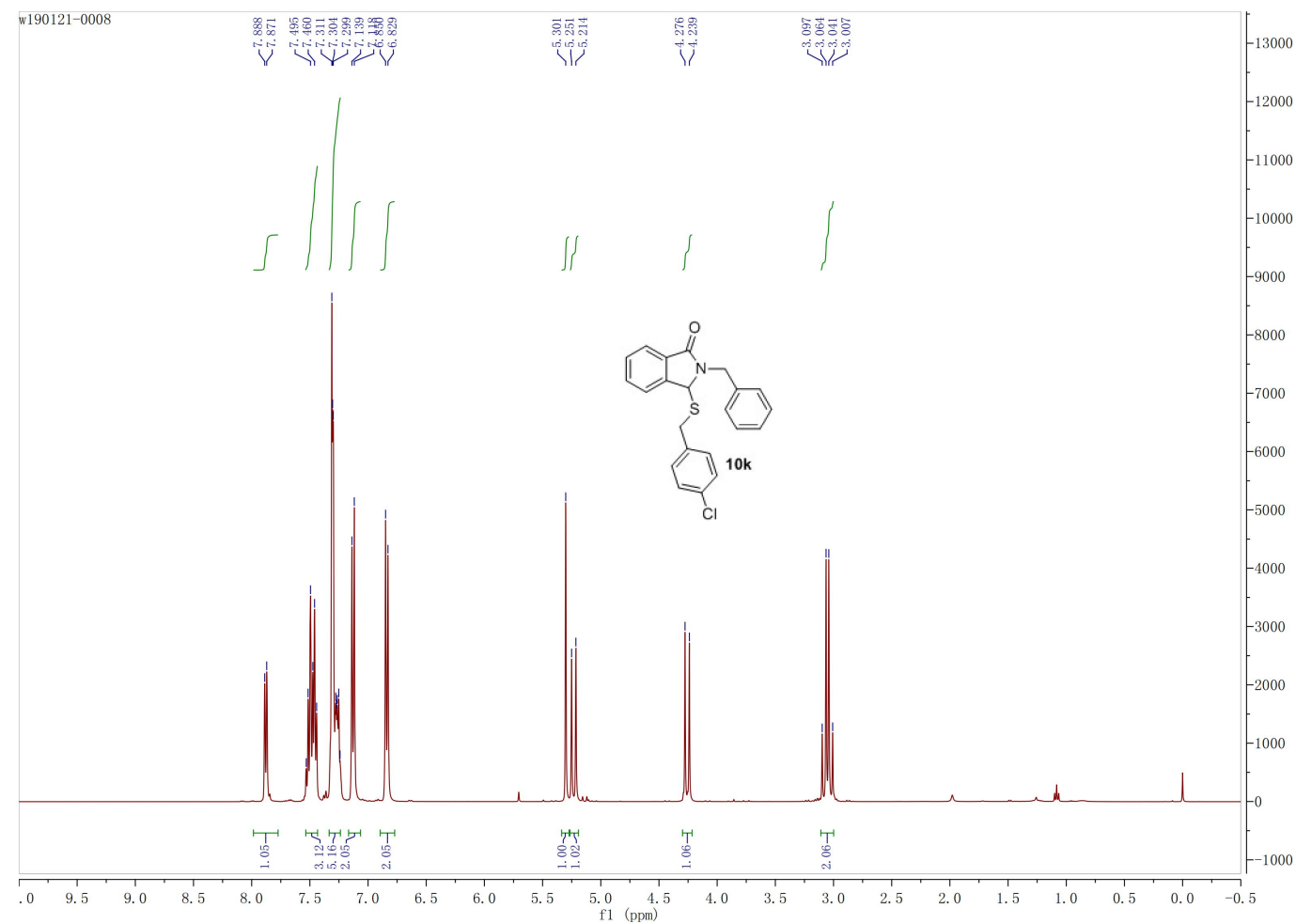

Figure S109. ${ }^{1} \mathrm{HNMR}\left(400 \mathrm{MHz}, \mathrm{CDCl}_{3}\right)$ of compound $10 \mathrm{k}$

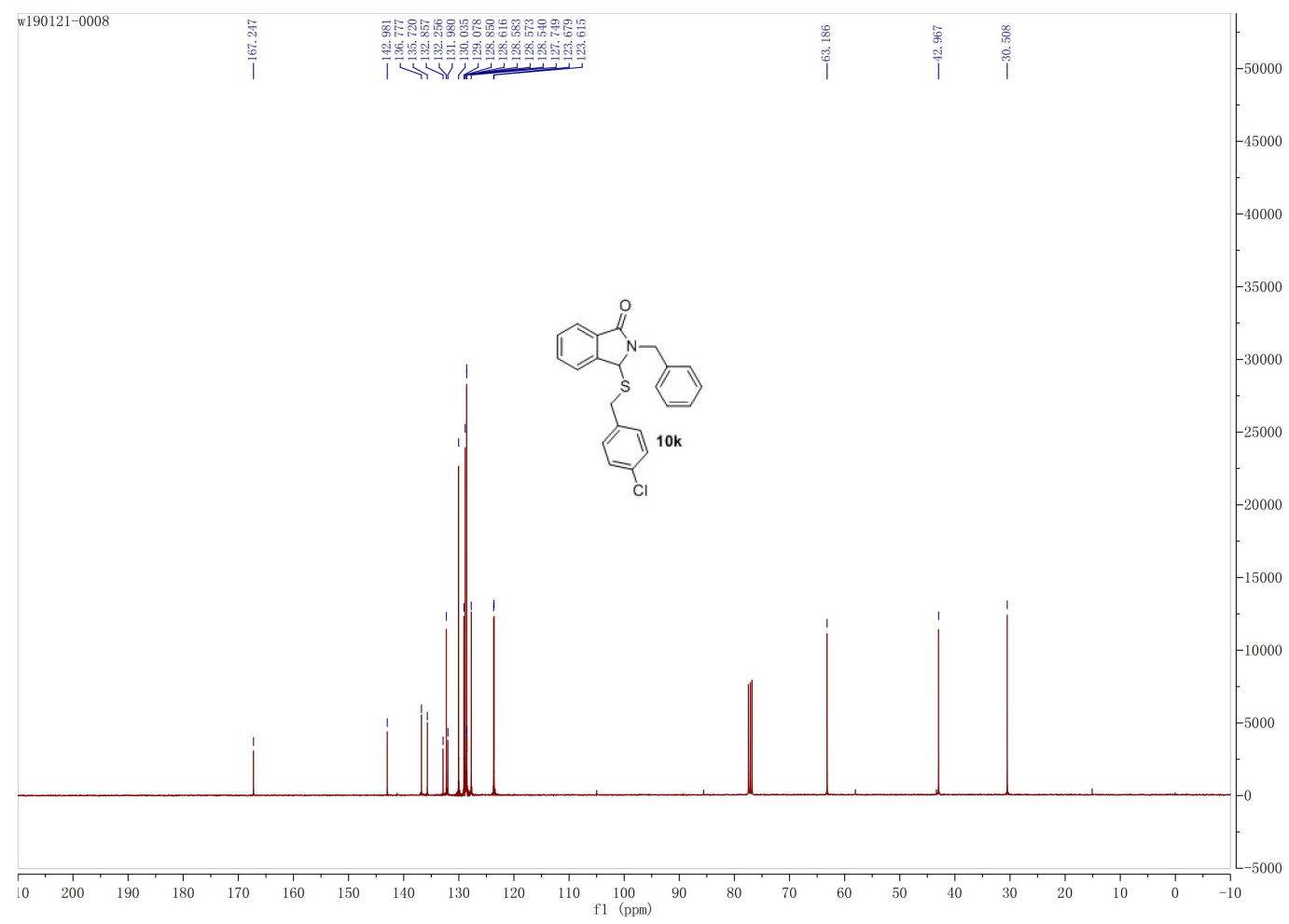

Figure S110. ${ }^{13} \mathrm{CNMR}\left(100 \mathrm{MHz}, \mathrm{CDCl}_{3}\right)$ of compound $10 \mathrm{k}$ 


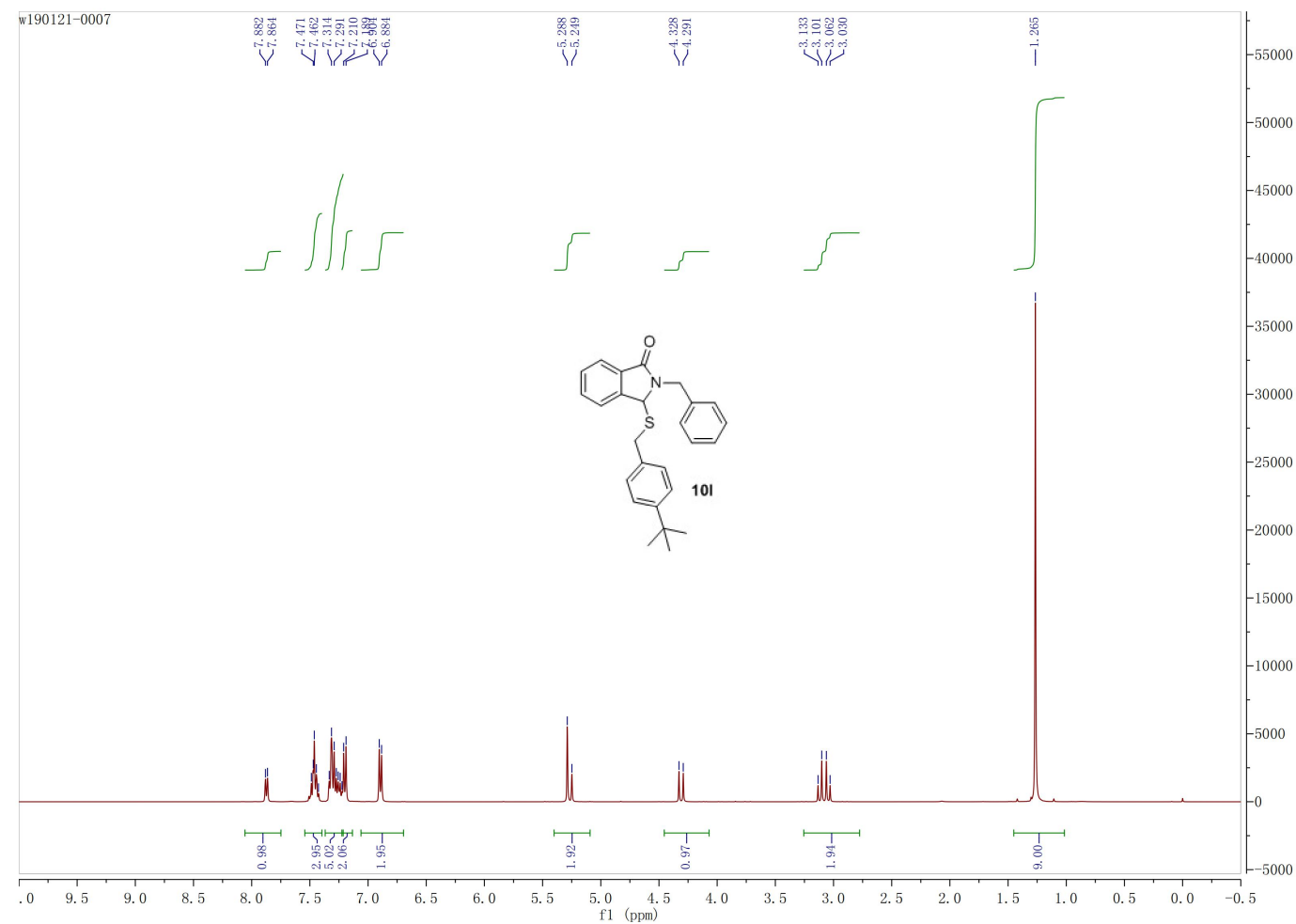

Figure S111. ${ }^{1} \mathrm{HNMR}\left(400 \mathrm{MHz}, \mathrm{CDCl}_{3}\right)$ of compound 101

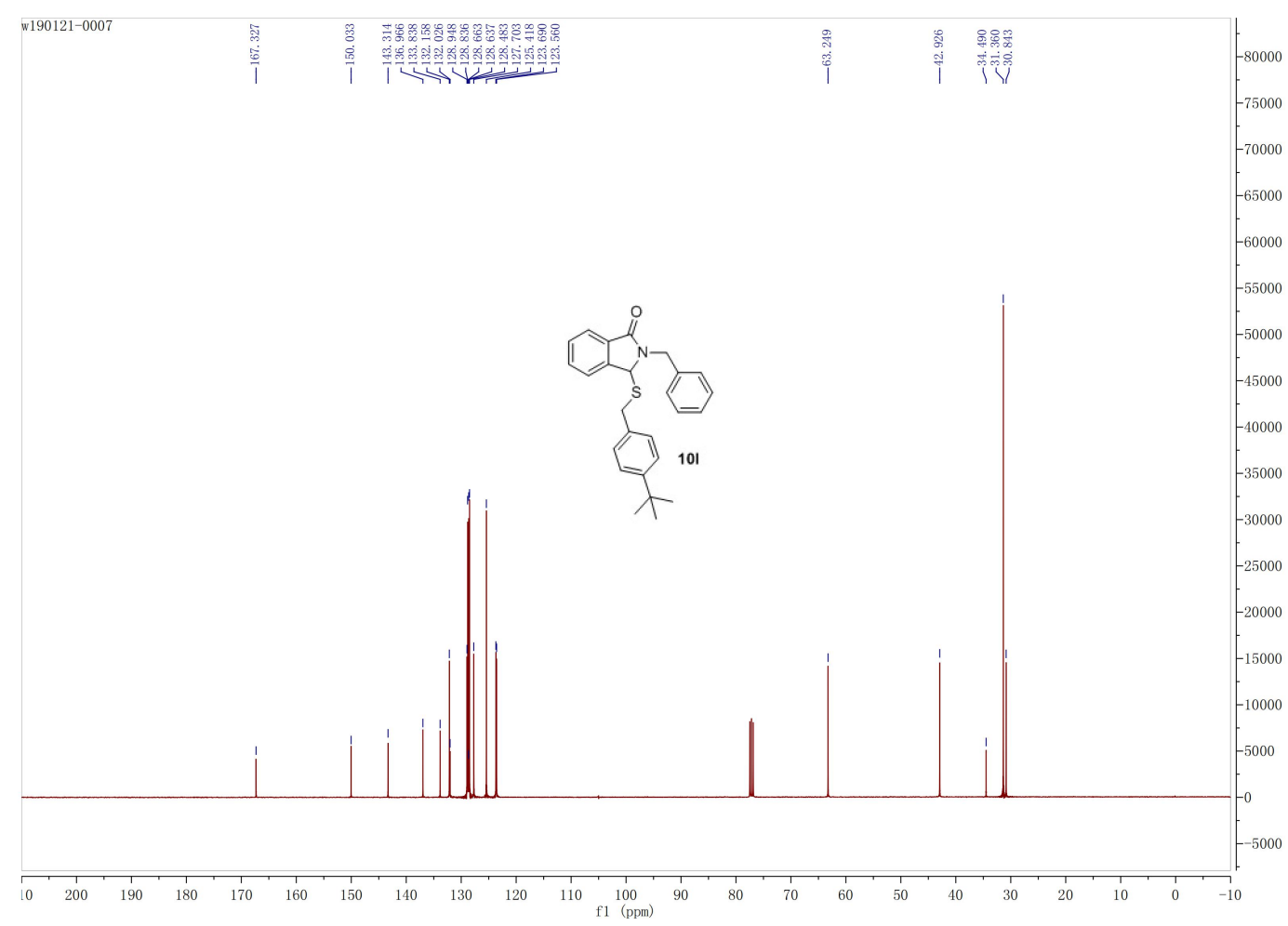

Figure S112. ${ }^{13} \mathrm{CNMR}\left(100 \mathrm{MHz}, \mathrm{CDCl}_{3}\right)$ of compound 101 


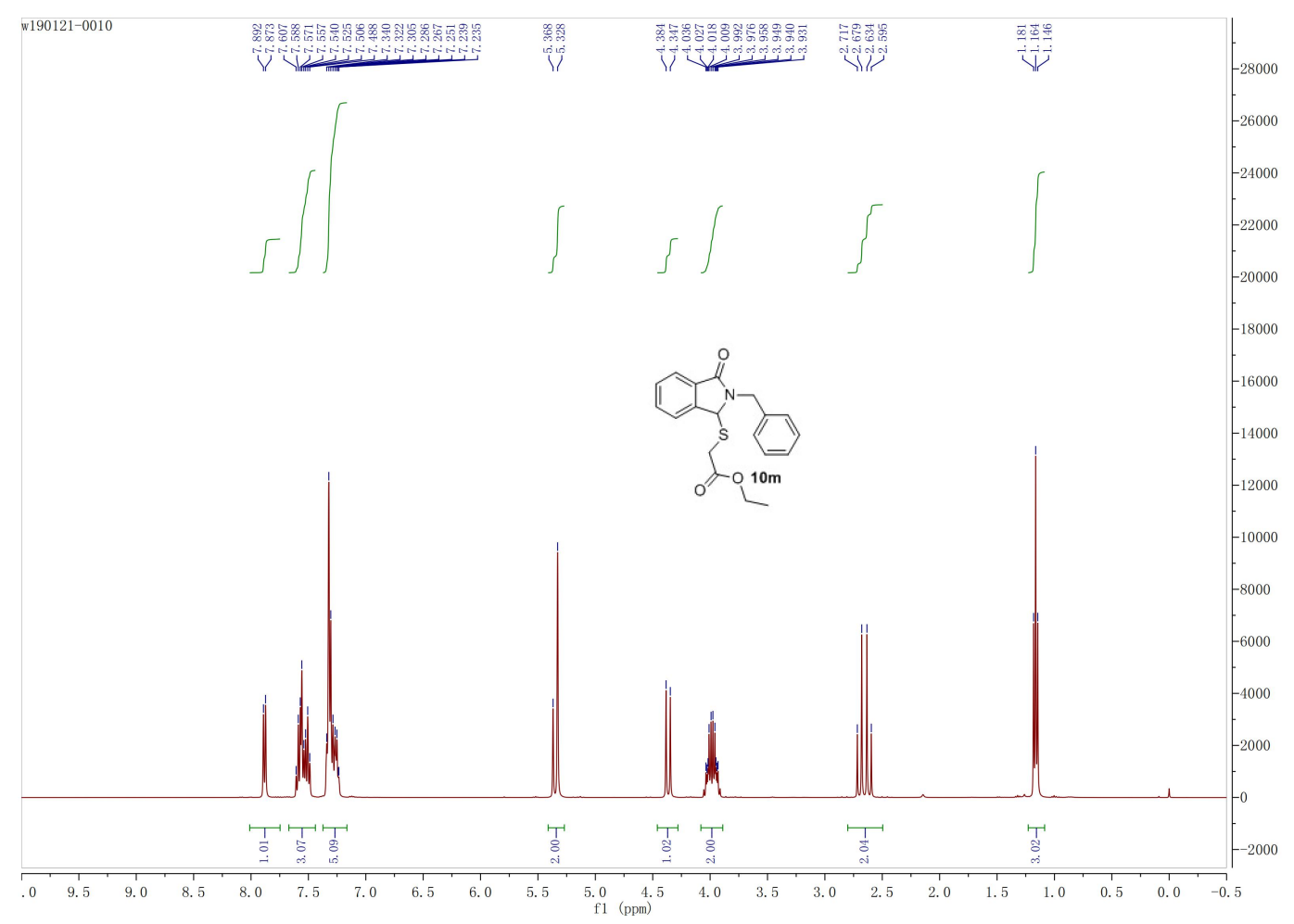

Figure S113. ${ }^{1} \mathrm{HNMR}\left(400 \mathrm{MHz}, \mathrm{CDCl}_{3}\right)$ of compound $10 \mathrm{~m}$

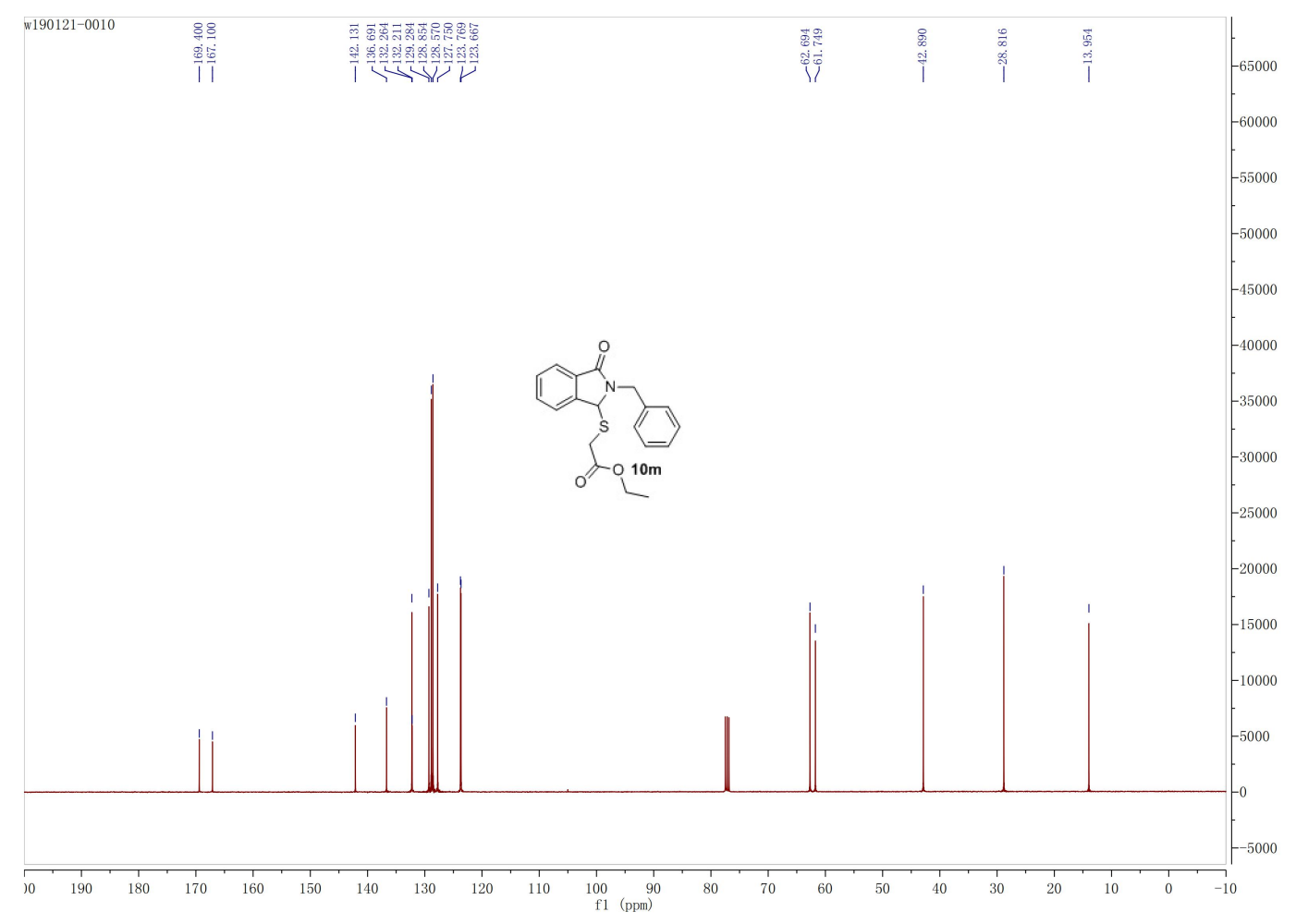

Figure S114. ${ }^{13} \mathrm{CNMR}\left(100 \mathrm{MHz}, \mathrm{CDCl}_{3}\right)$ of compound $10 \mathrm{~m}$ 


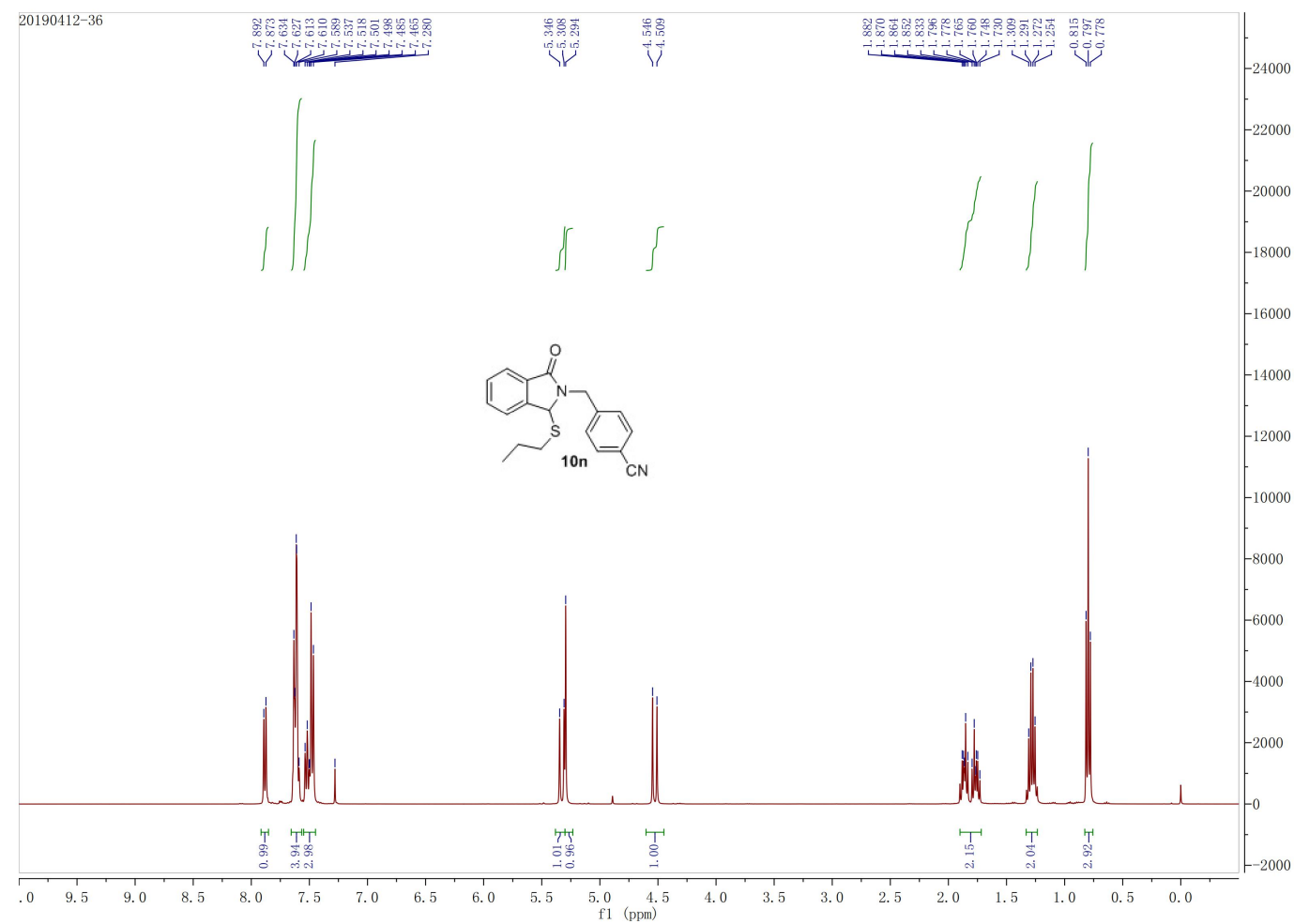

Figure S115. ${ }^{1} \mathrm{HNMR}\left(400 \mathrm{MHz}, \mathrm{CDCl}_{3}\right)$ of compound $10 \mathrm{n}$

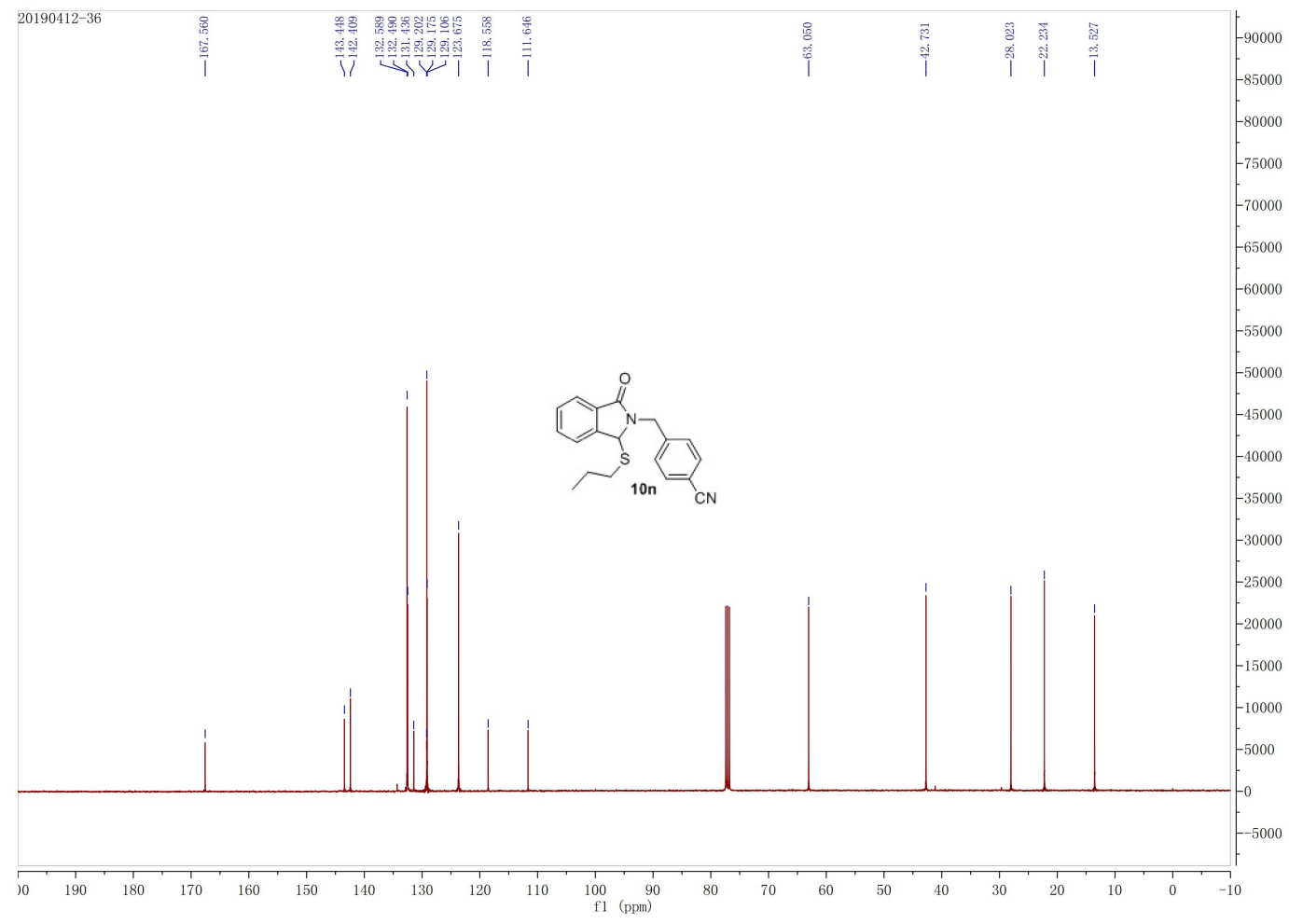

Figure S116. ${ }^{13} \mathrm{CNMR}\left(100 \mathrm{MHz}, \mathrm{CDCl}_{3}\right)$ of compound $10 \mathrm{n}$ 


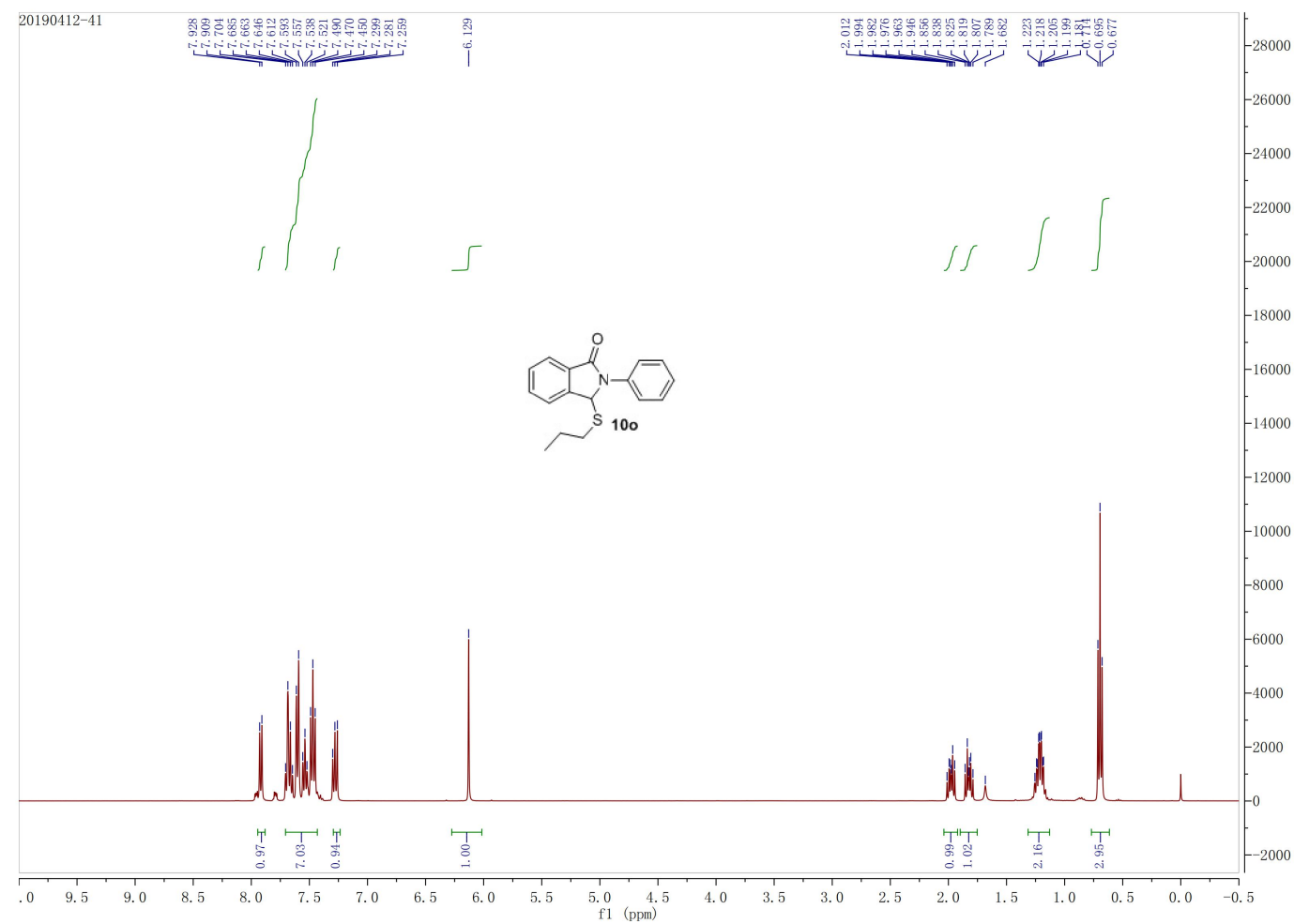

Figure S117. ${ }^{1} \mathrm{HNMR}\left(400 \mathrm{MHz}, \mathrm{CDCl}_{3}\right)$ of compound $10 \mathrm{o}$

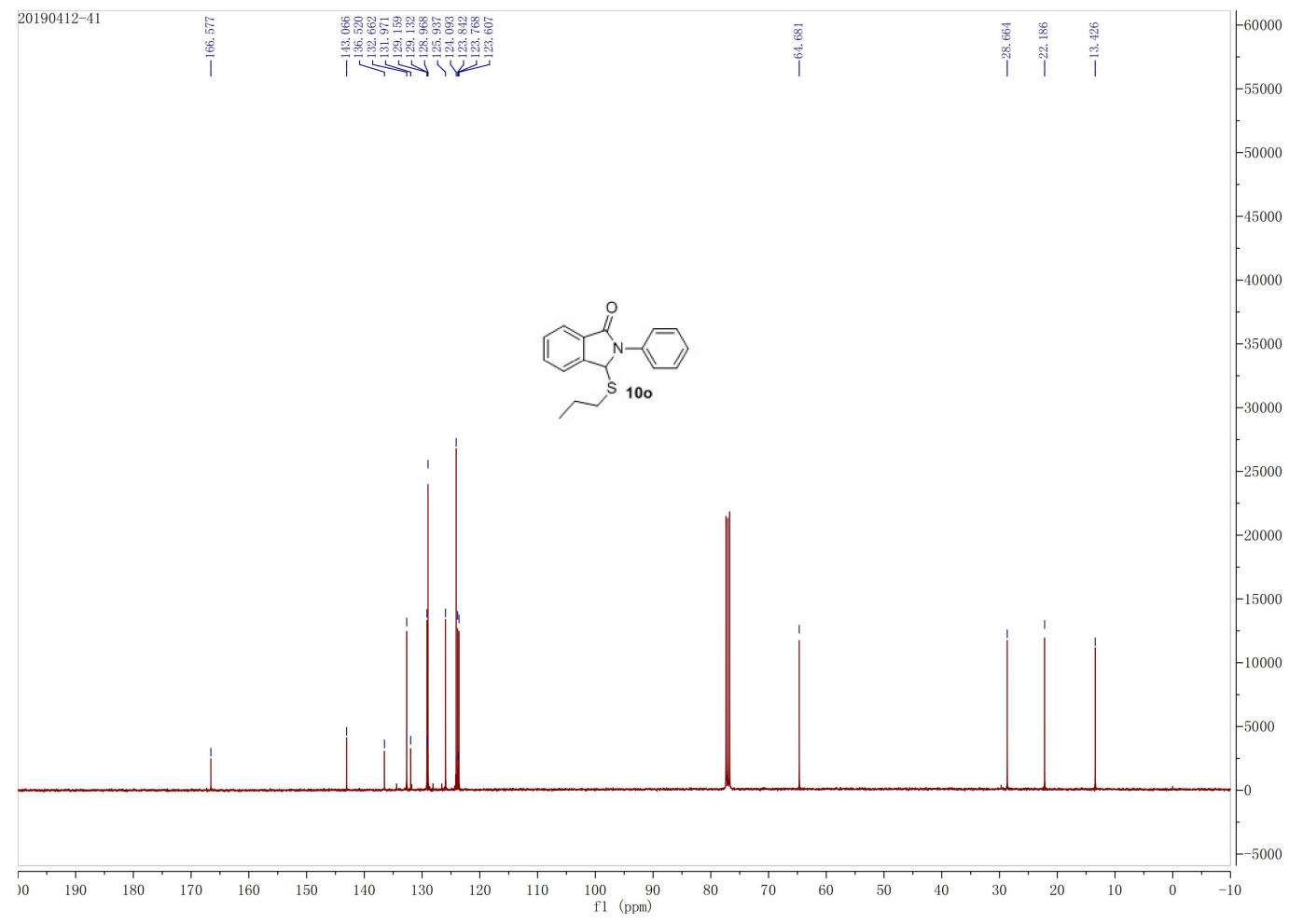

Figure S118. ${ }^{13} \mathrm{CNMR}\left(100 \mathrm{MHz}, \mathrm{CDCl}_{3}\right)$ of compound $10 \mathrm{o}$ 


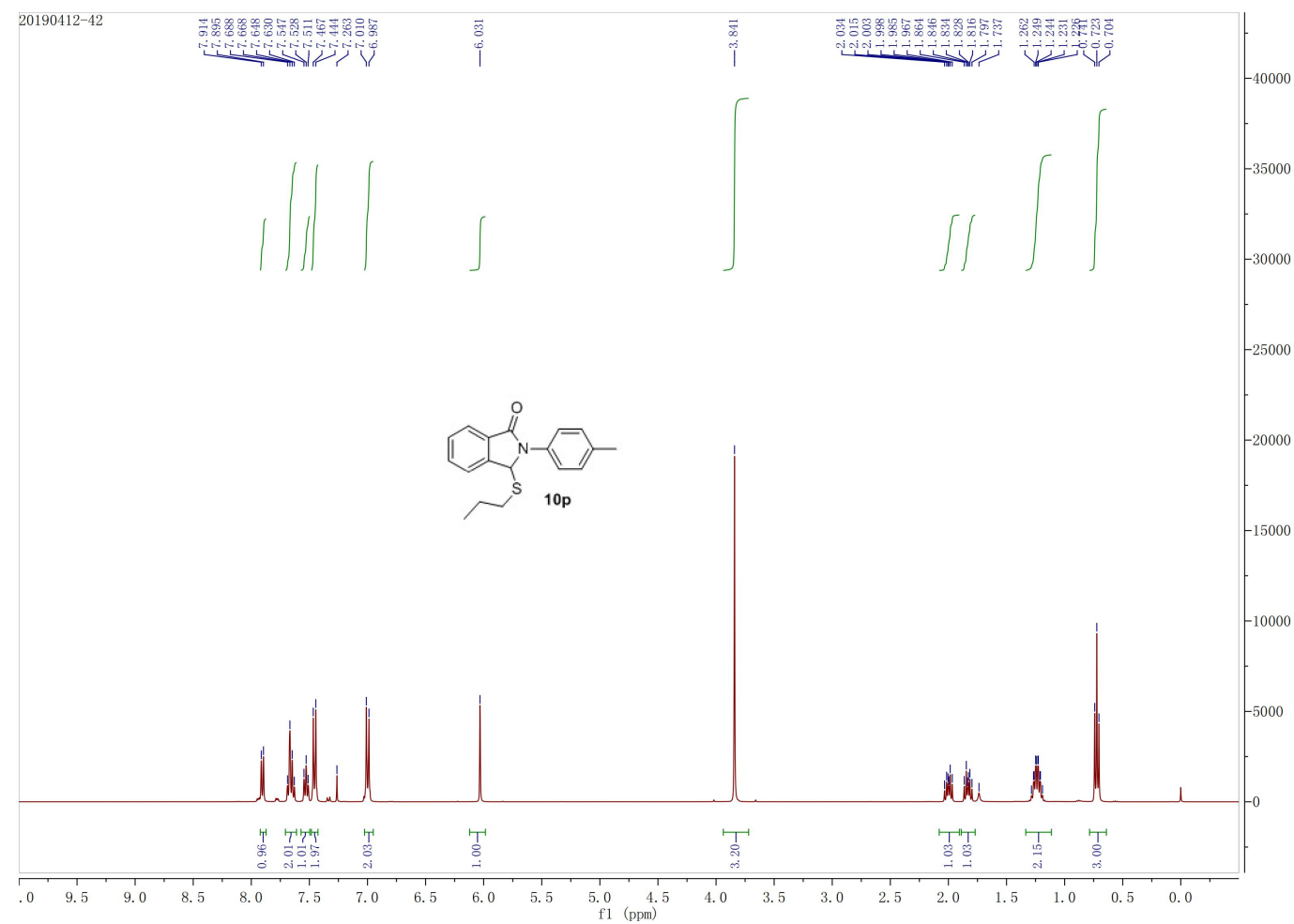

Figure S119. ${ }^{1} \mathrm{HNMR}\left(400 \mathrm{MHz}, \mathrm{CDCl}_{3}\right)$ of compound $10 \mathrm{p}$

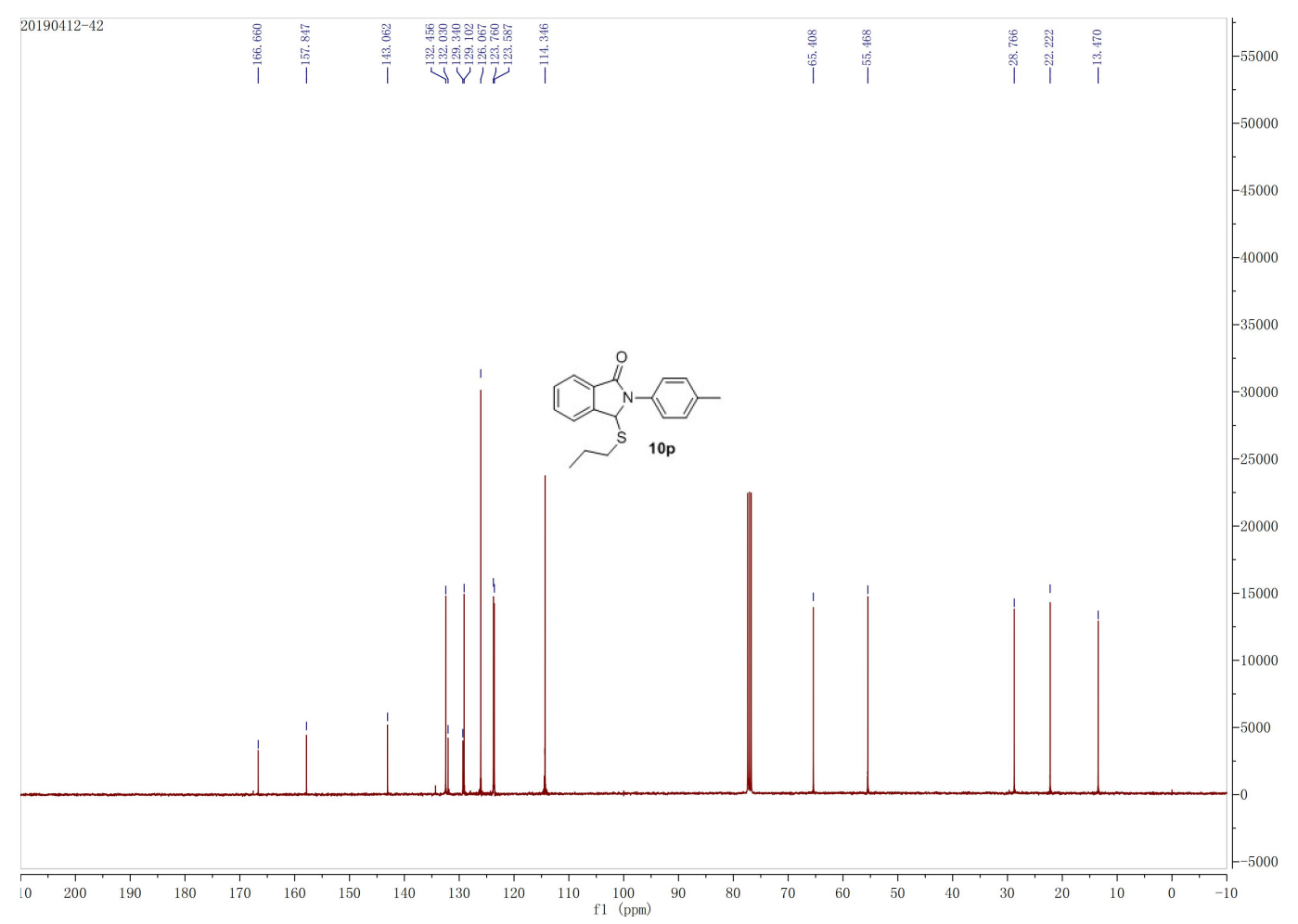

Figure S120. ${ }^{13} \mathrm{CNMR}\left(100 \mathrm{MHz}, \mathrm{CDCl}_{3}\right)$ of compound $10 \mathrm{p}$ 


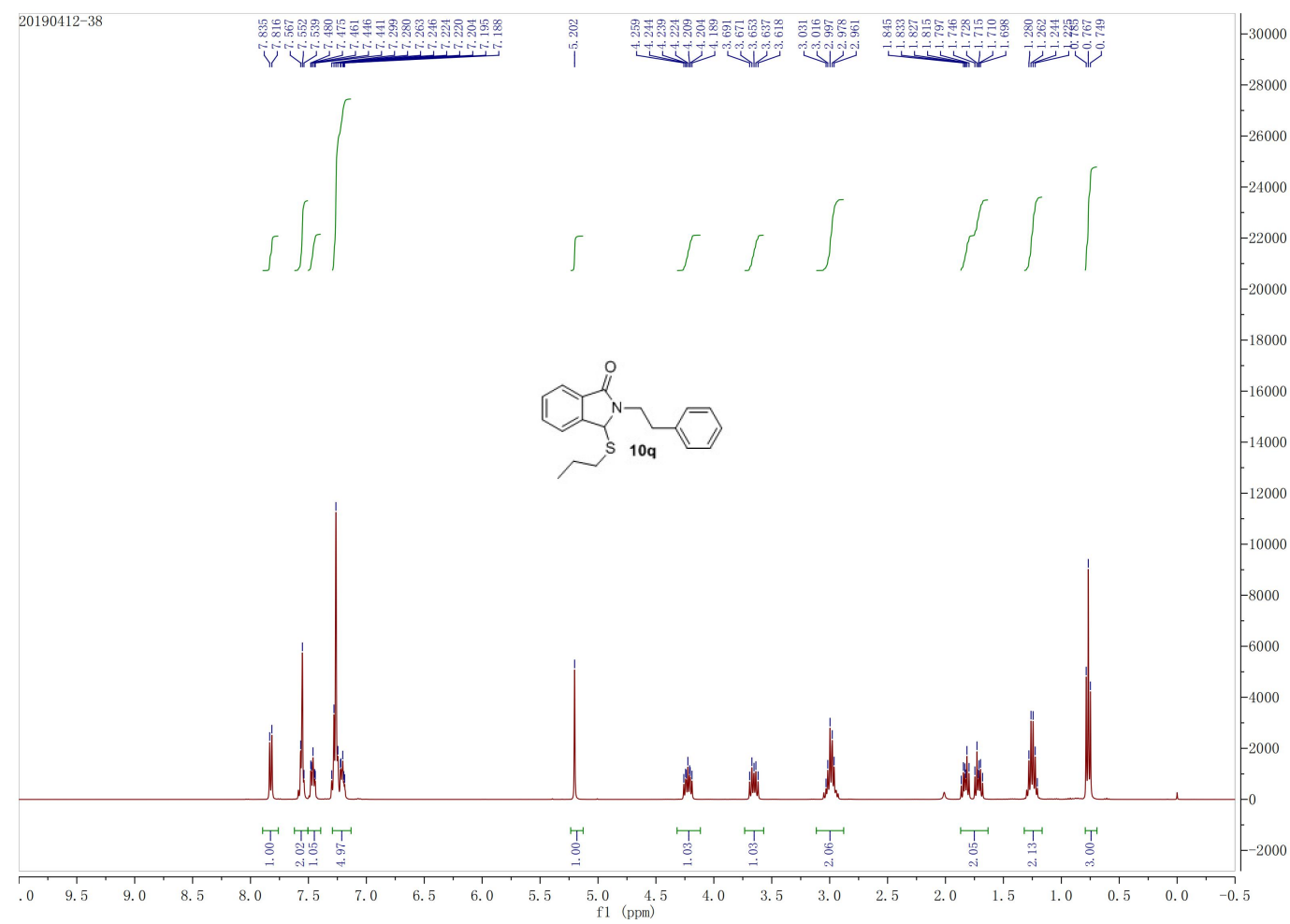

Figure S121. ${ }^{1} \mathrm{HNMR}\left(400 \mathrm{MHz}, \mathrm{CDCl}_{3}\right)$ of compound $10 \mathrm{q}$

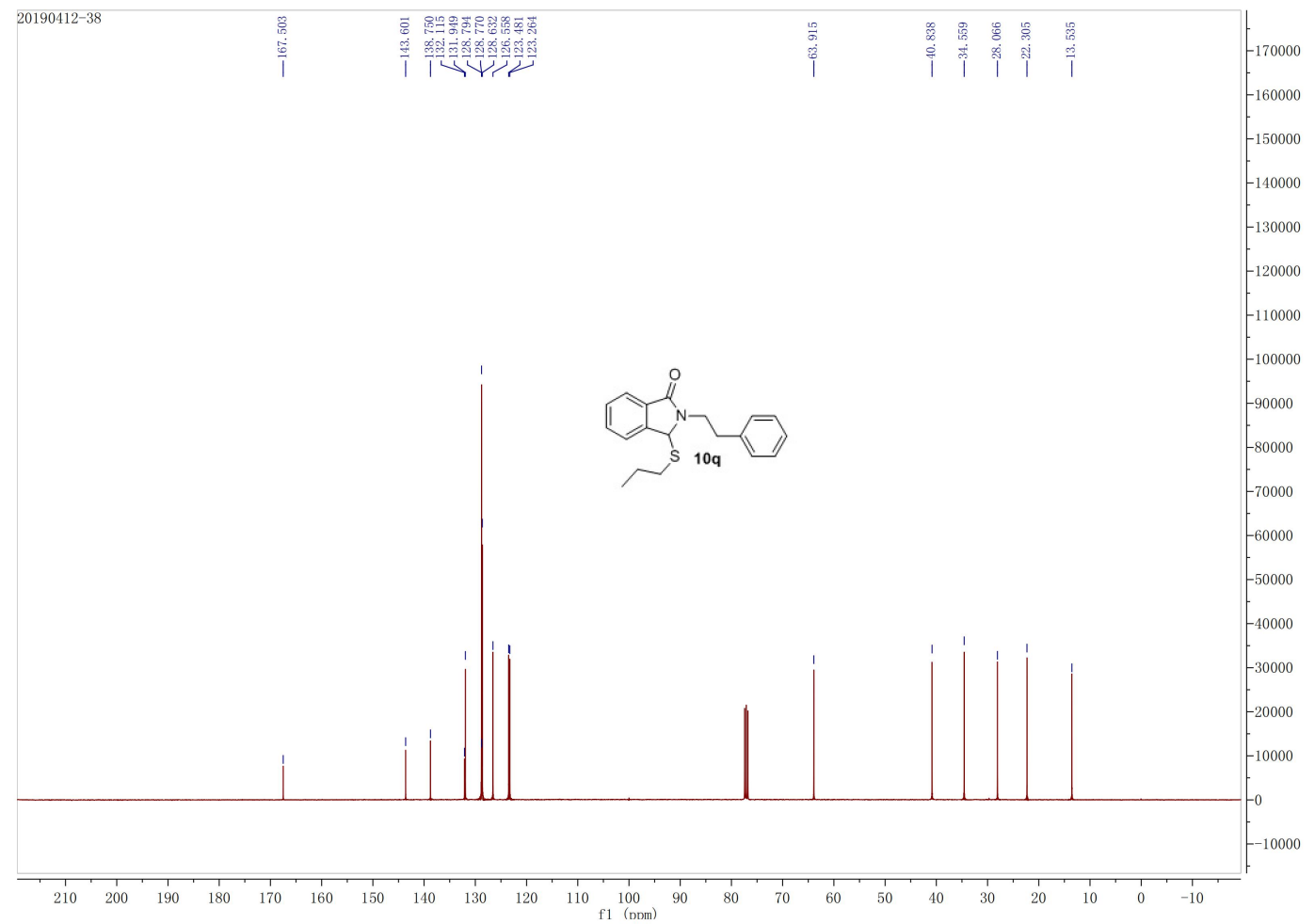

Figure S122. ${ }^{13} \mathrm{CNMR}\left(100 \mathrm{MHz}, \mathrm{CDCl}_{3}\right)$ of compound 10q 


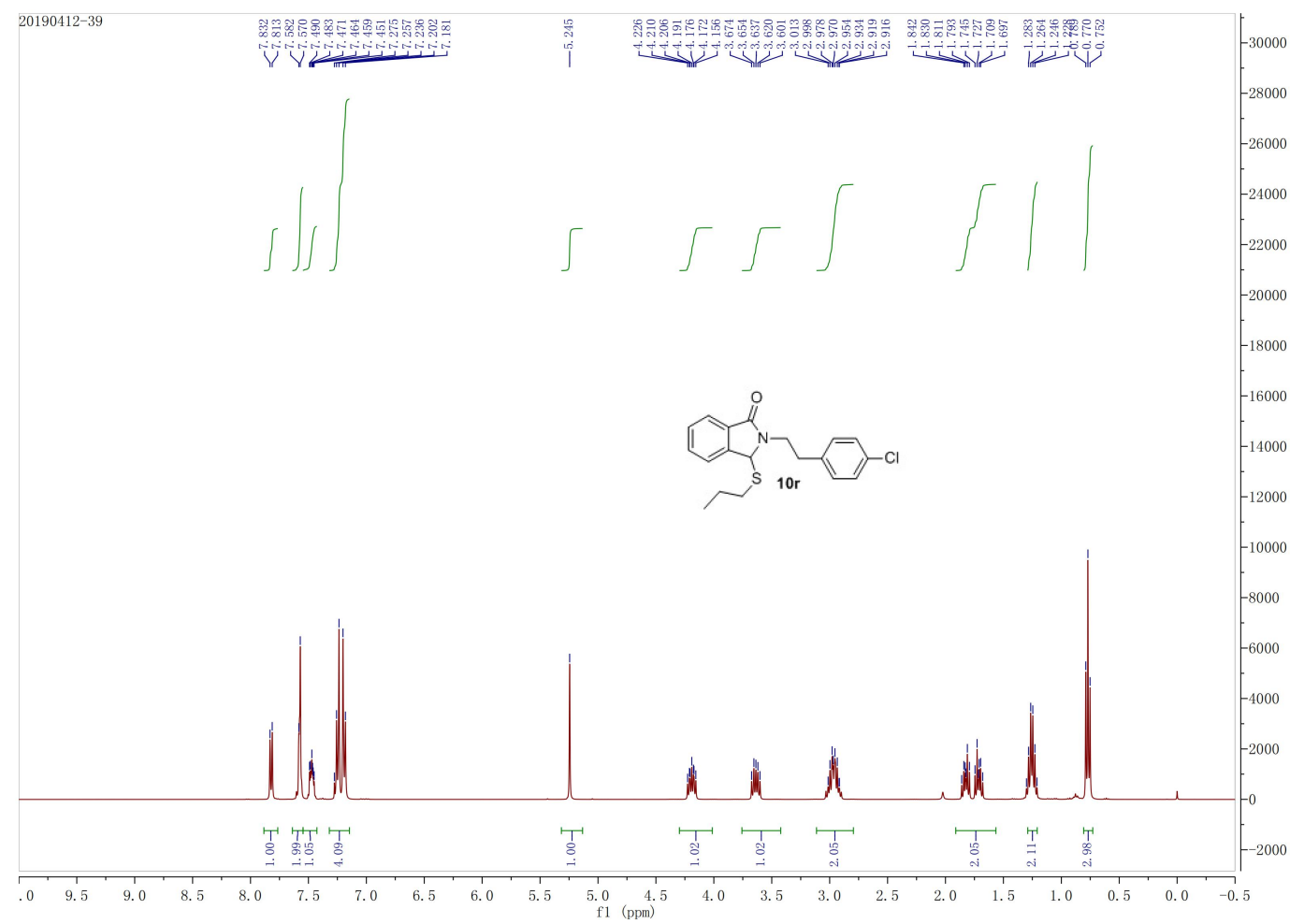

Figure S123. ${ }^{1} \mathrm{HNMR}\left(400 \mathrm{MHz}, \mathrm{CDCl}_{3}\right)$ of compound $10 \mathrm{r}$

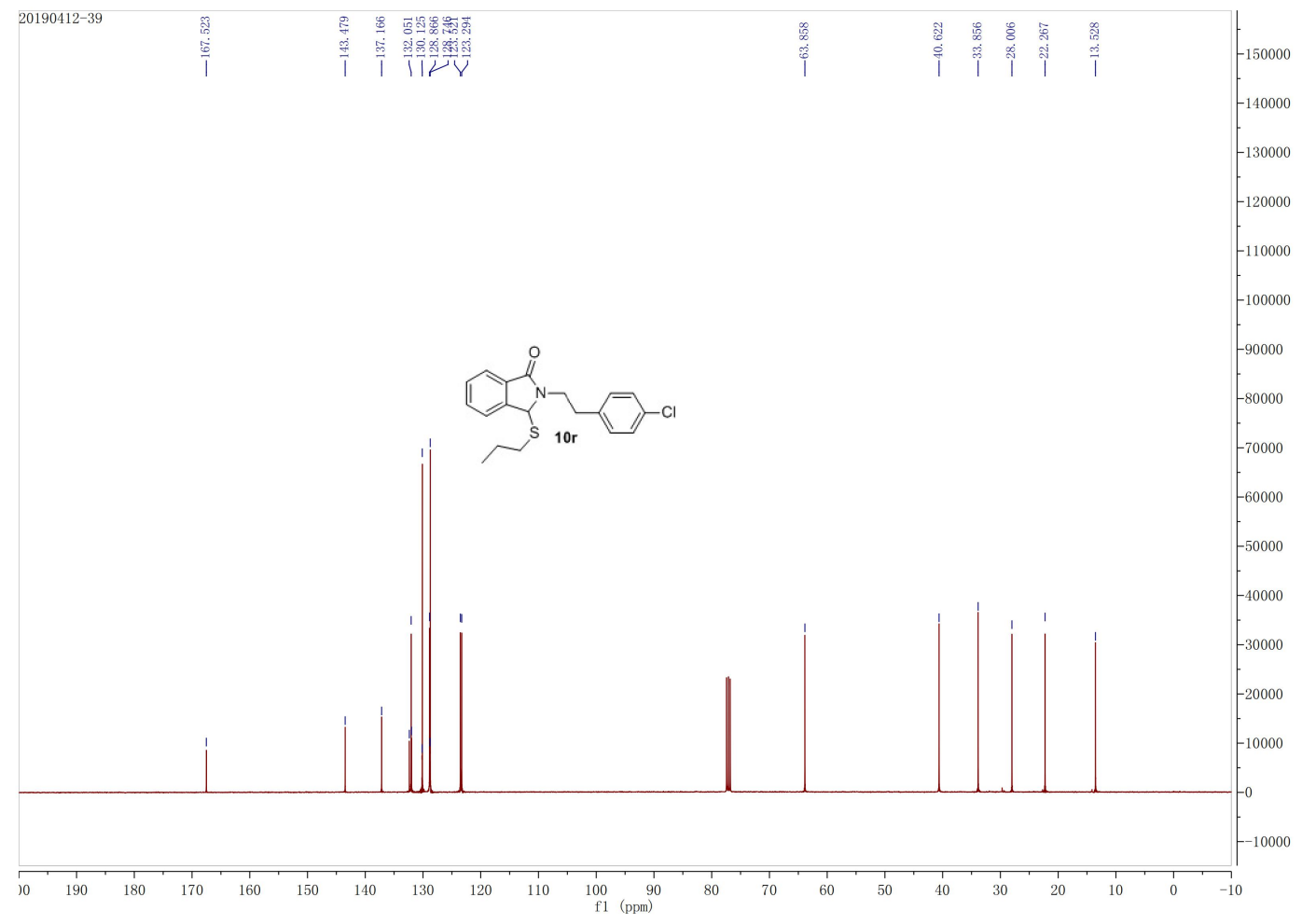

Figure S124. ${ }^{13} \mathrm{CNMR}\left(100 \mathrm{MHz}, \mathrm{CDCl}_{3}\right)$ of compound $10 \mathrm{r}$ 


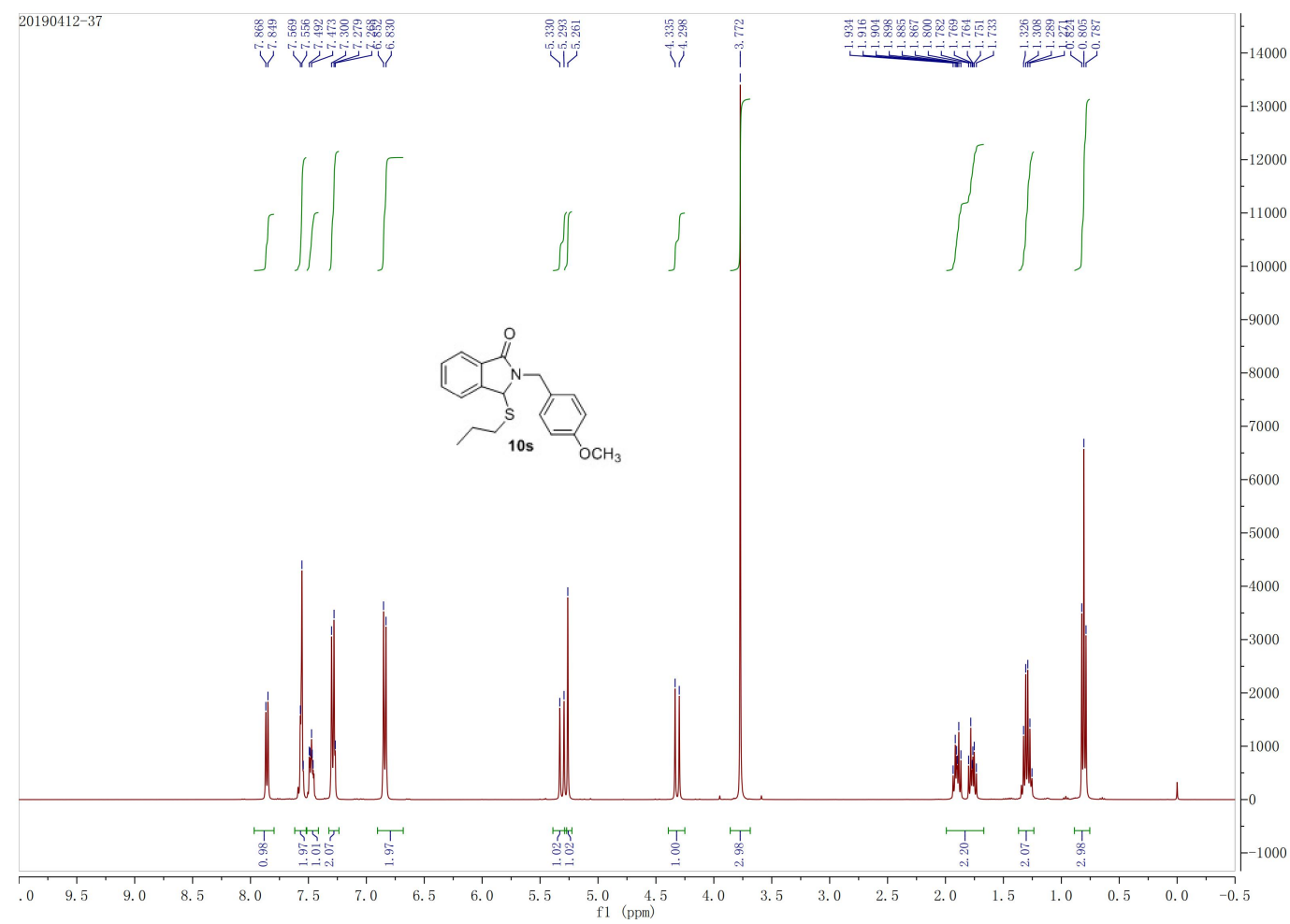

Figure S125. ${ }^{1} \mathrm{HNMR}\left(400 \mathrm{MHz}, \mathrm{CDCl}_{3}\right)$ of compound $10 \mathrm{~s}$

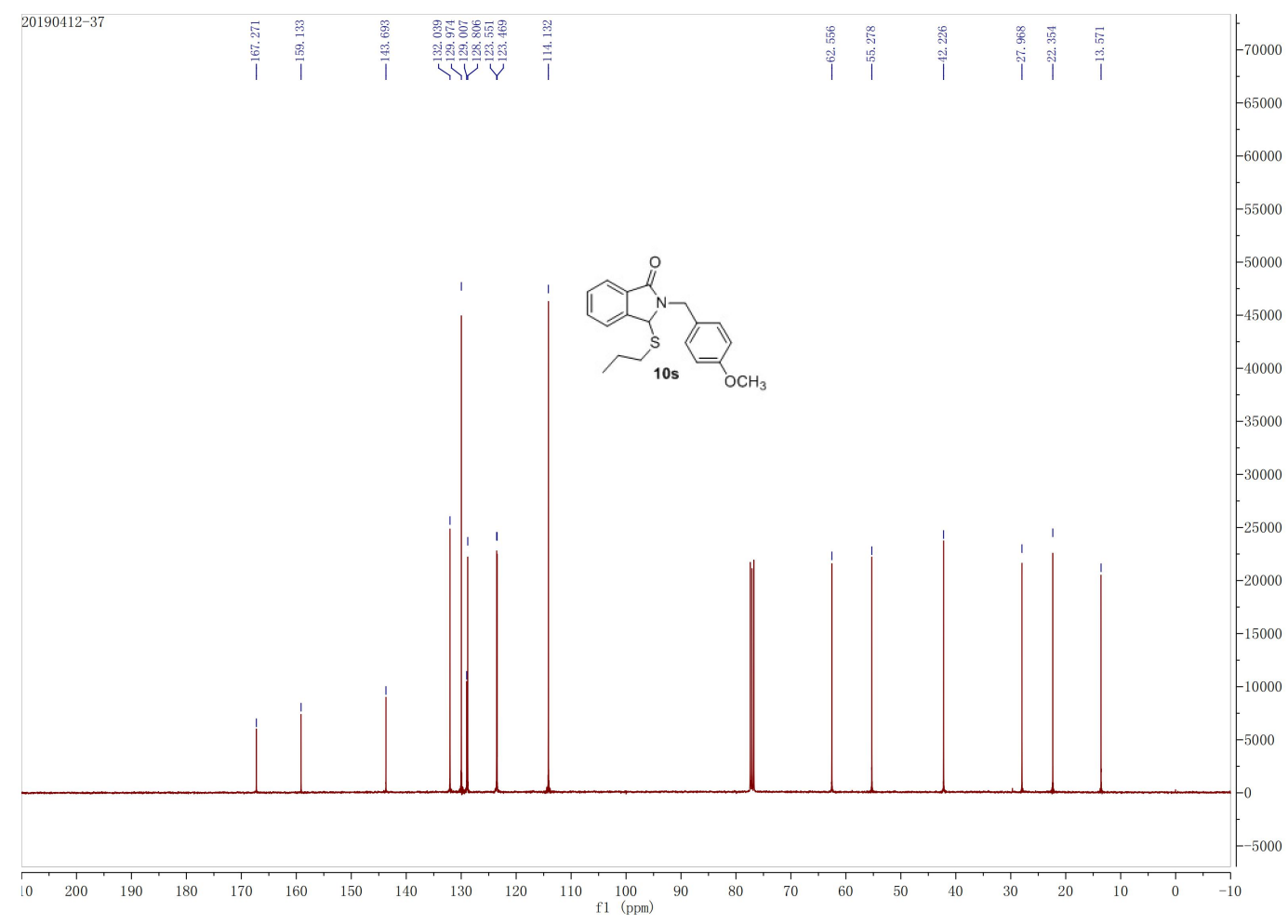

Figure S126. ${ }^{13} \mathrm{CNMR}\left(100 \mathrm{MHz}, \mathrm{CDCl}_{3}\right)$ of compound $10 \mathrm{~s}$ 


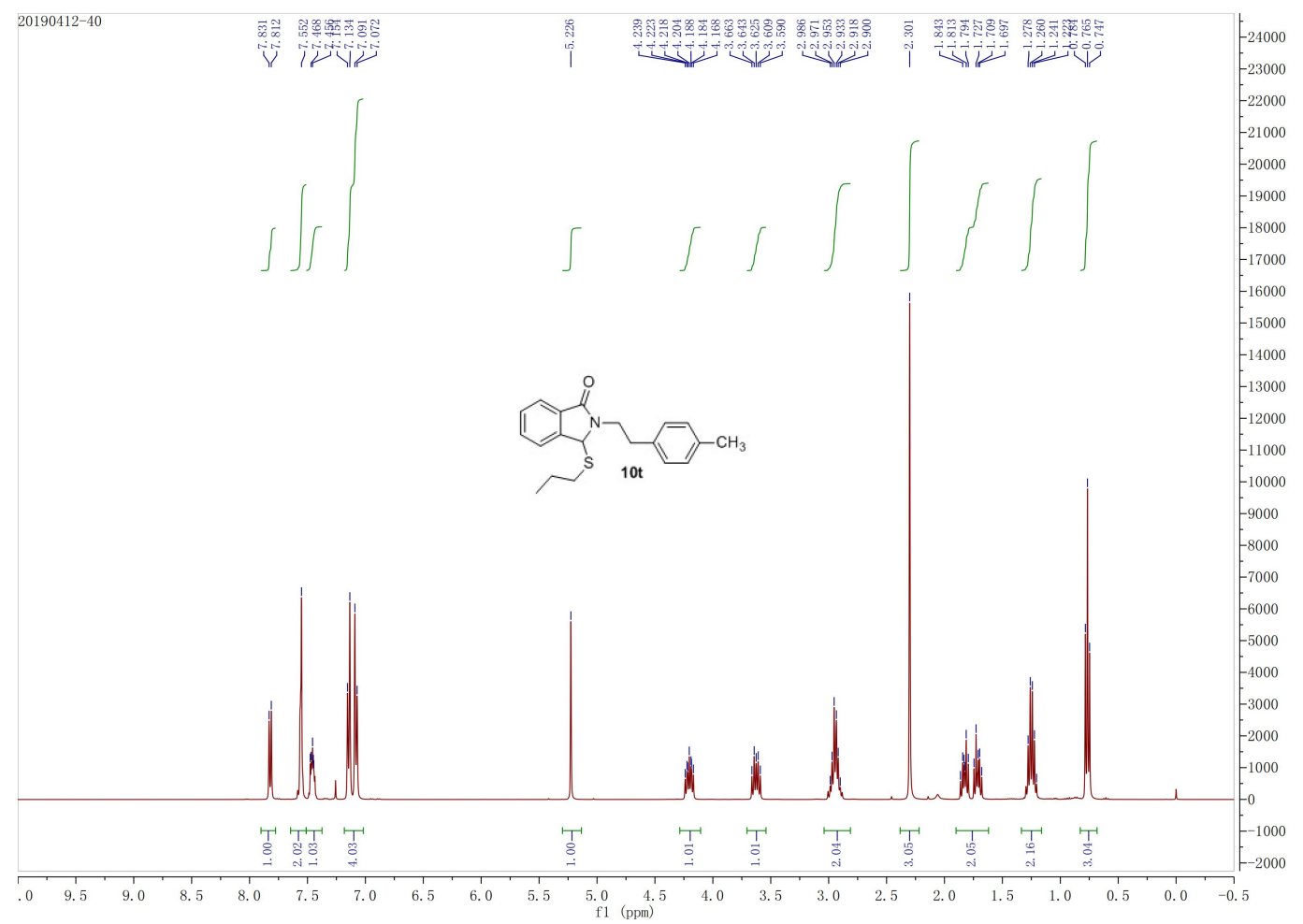

Figure S127. ${ }^{1} \mathrm{HNMR}\left(400 \mathrm{MHz}, \mathrm{CDCl}_{3}\right)$ of compound $10 \mathrm{t}$

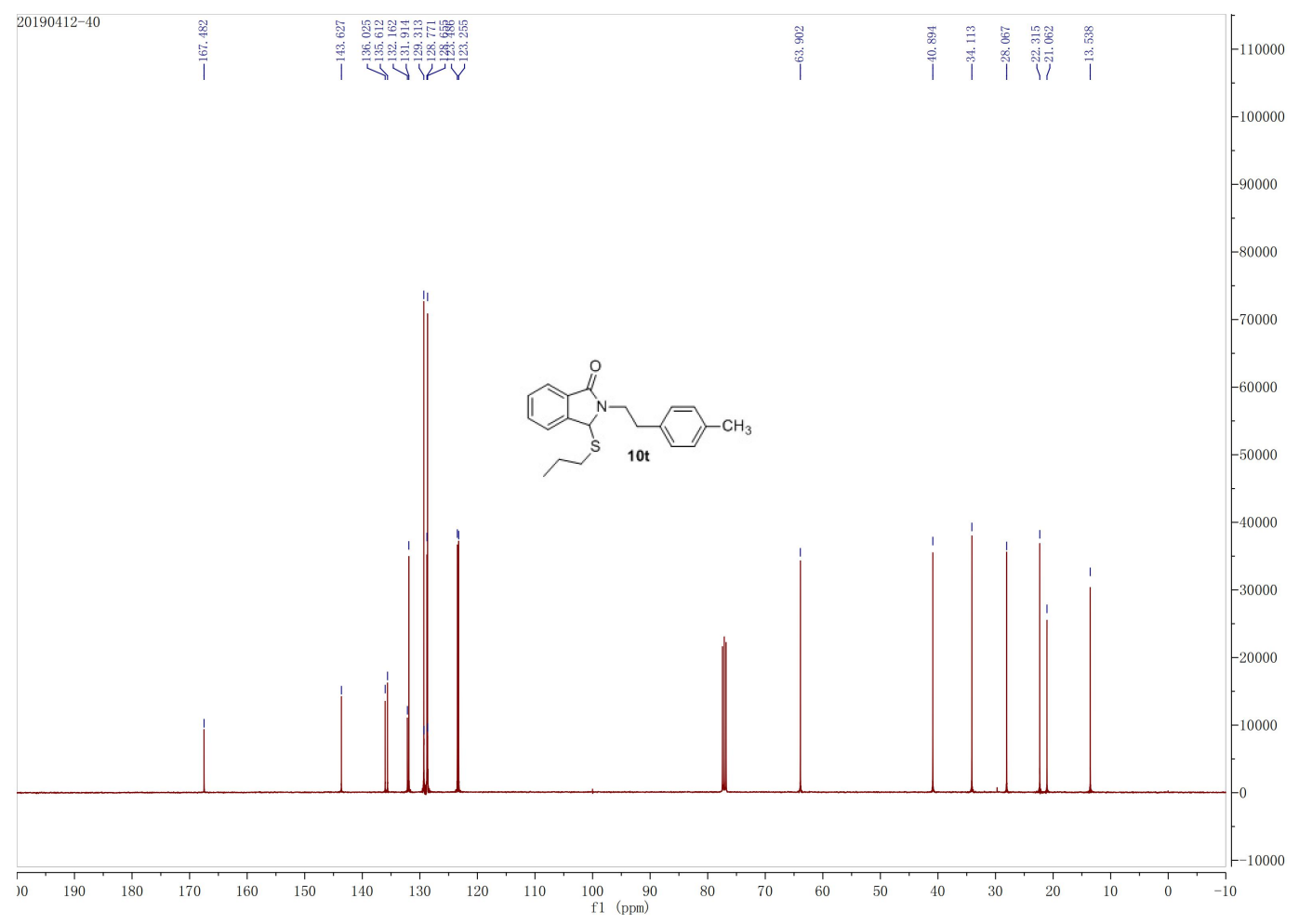

Figure S128. ${ }^{13} \mathrm{CNMR}\left(100 \mathrm{MHz}, \mathrm{CDCl}_{3}\right)$ of compound $10 \mathrm{t}$ 


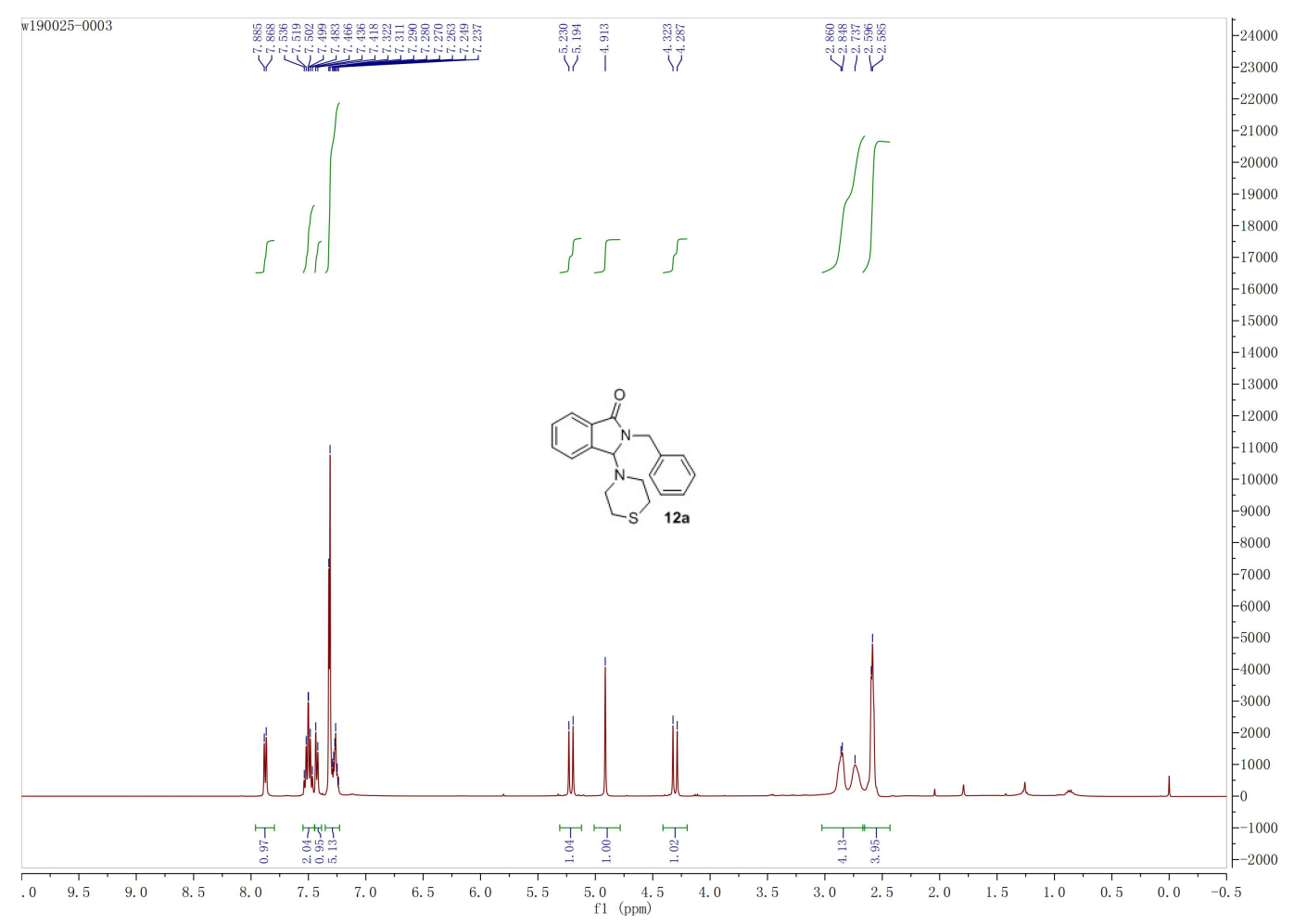

Figure S129. ${ }^{1} \mathrm{HNMR}\left(400 \mathrm{MHz}, \mathrm{CDCl}_{3}\right)$ of compound 12a

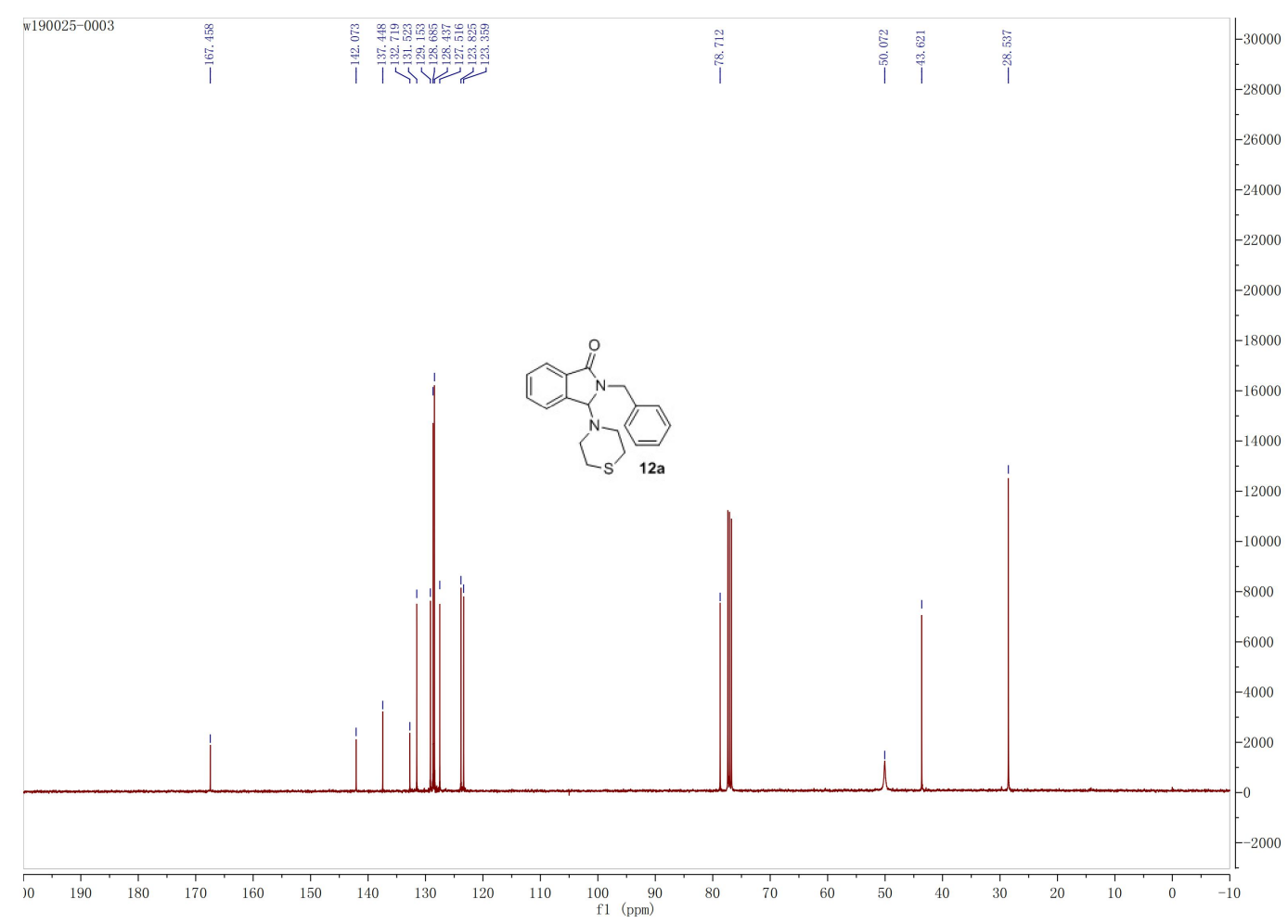

Figure S130. ${ }^{13} \mathrm{CNMR}\left(100 \mathrm{MHz}, \mathrm{CDCl}_{3}\right)$ of compound 12a 


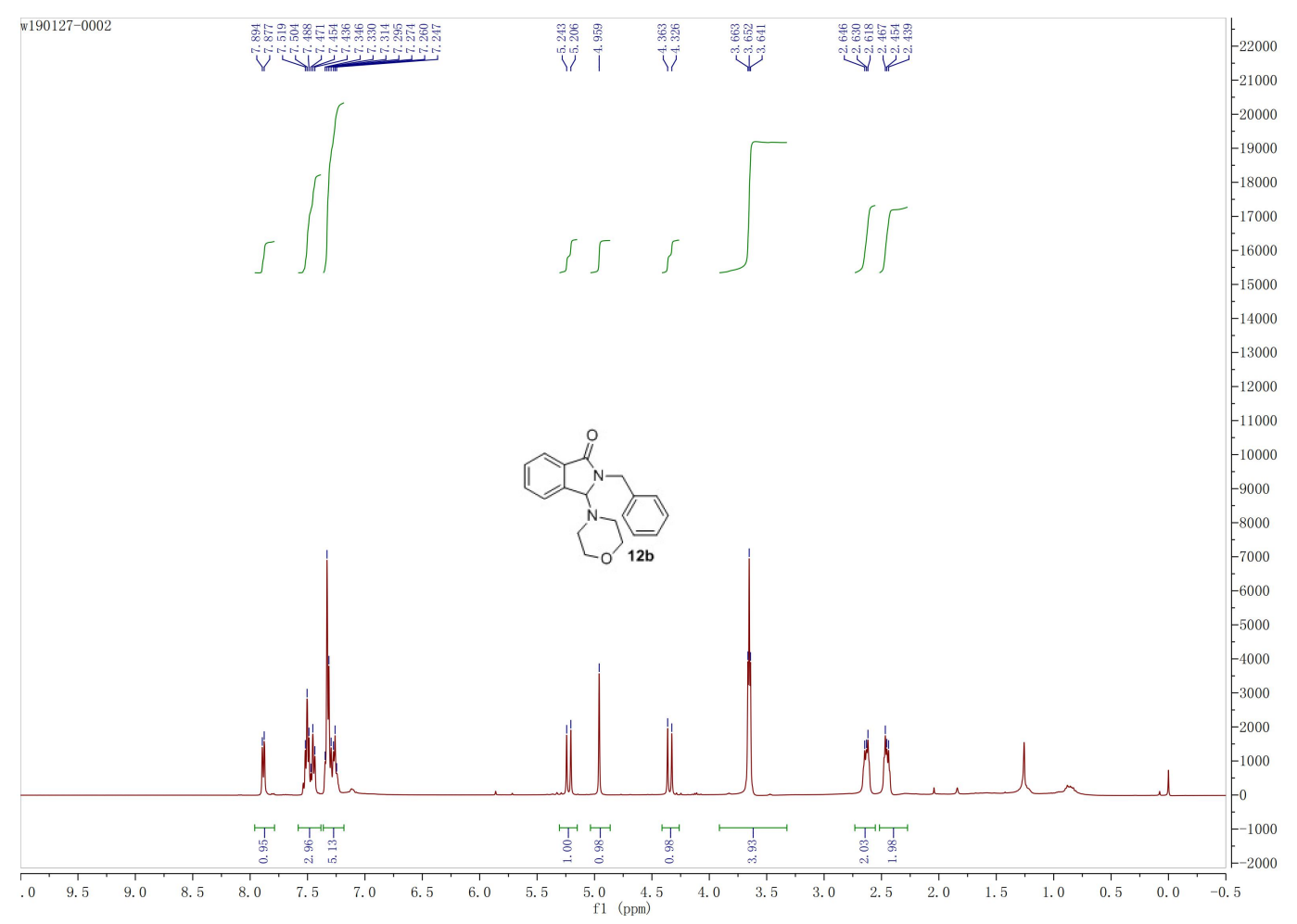

Figure S131. ${ }^{1} \mathrm{HNMR}\left(400 \mathrm{MHz}, \mathrm{CDCl}_{3}\right)$ of compound $12 \mathrm{~b}$

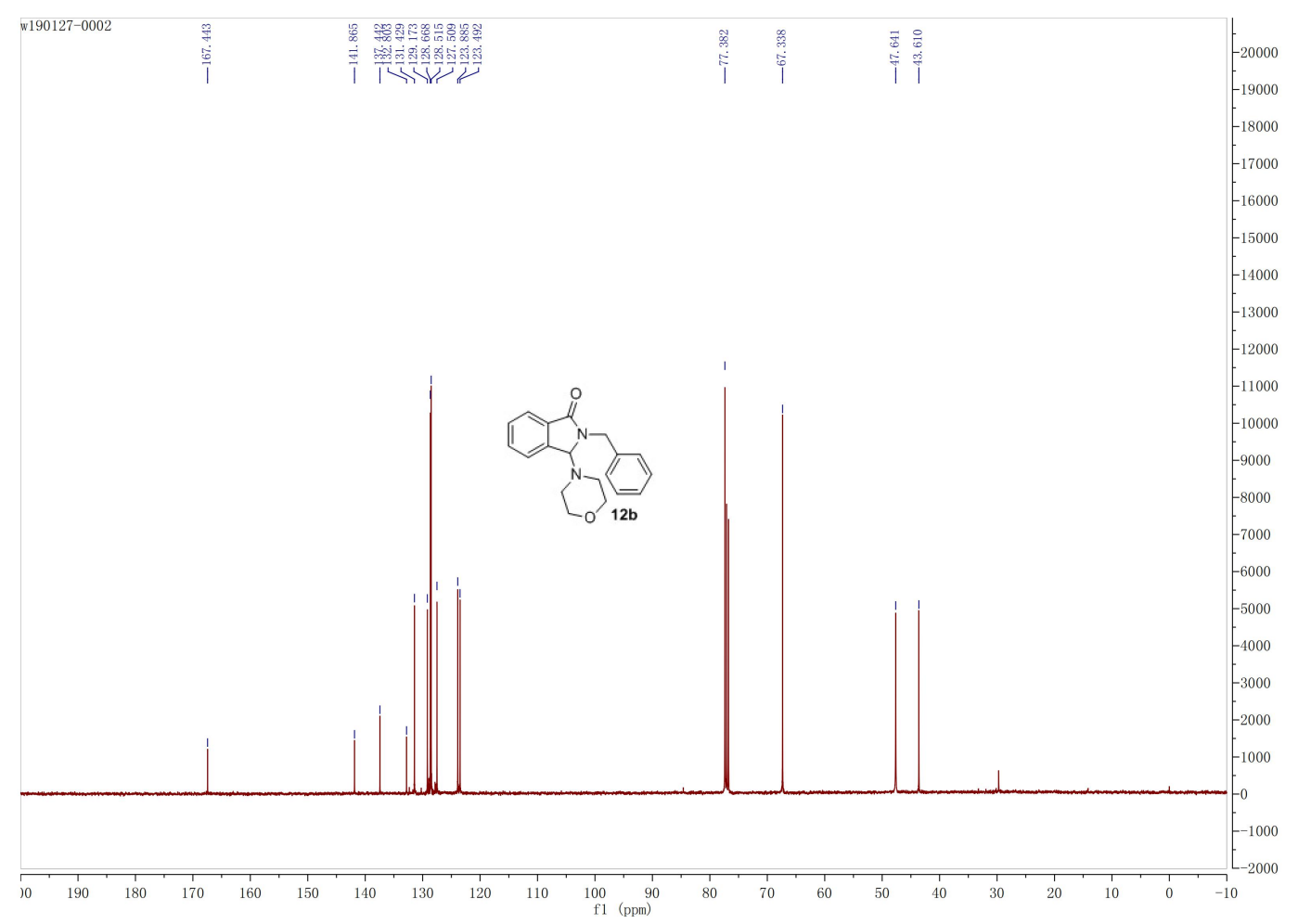

Figure S132. ${ }^{13} \mathrm{CNMR}\left(100 \mathrm{MHz}, \mathrm{CDCl}_{3}\right)$ of compound $12 \mathrm{~b}$ 


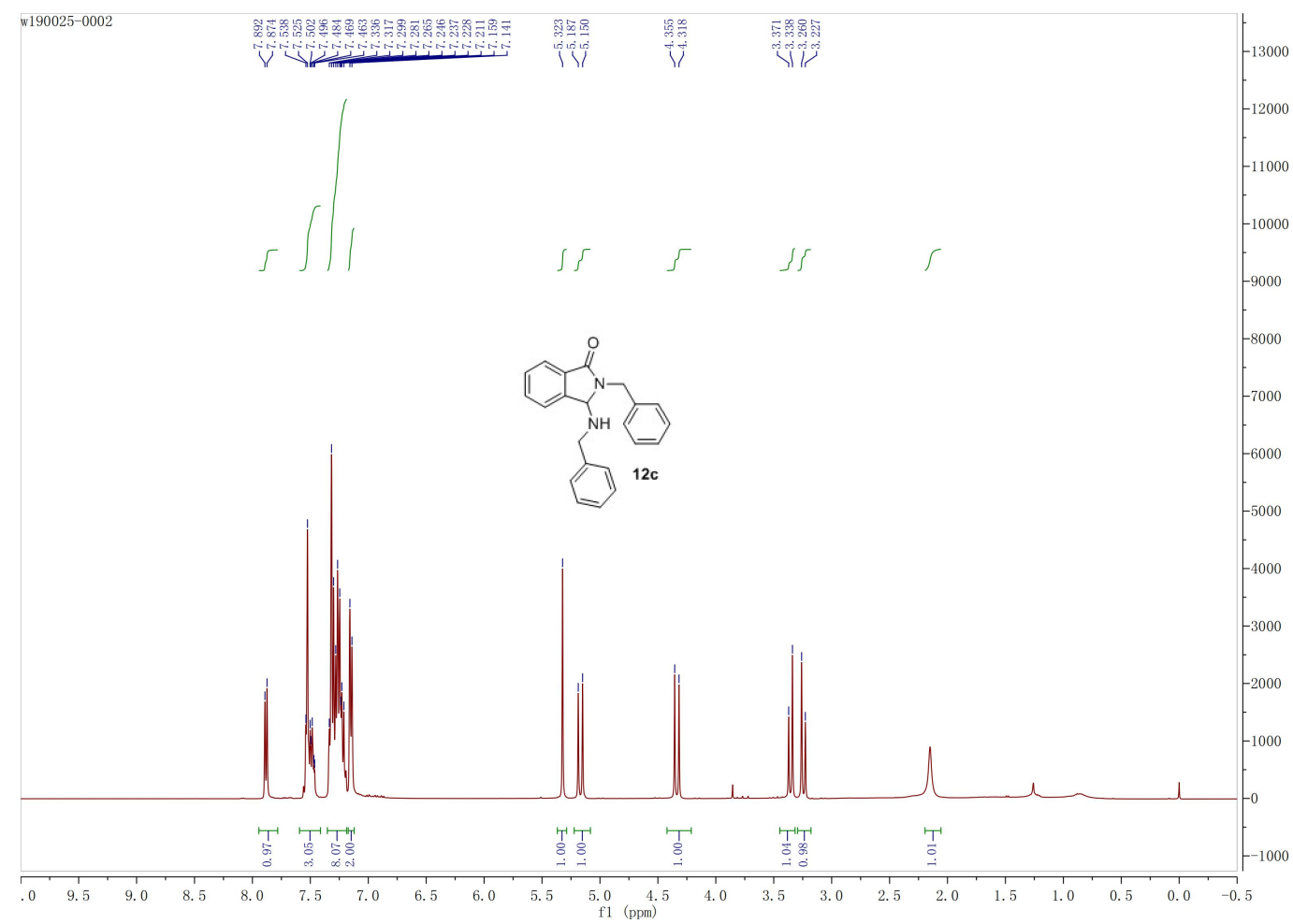

Figure S133. ${ }^{1} \mathrm{HNMR}\left(400 \mathrm{MHz}, \mathrm{CDCl}_{3}\right)$ of compound $12 \mathrm{c}$

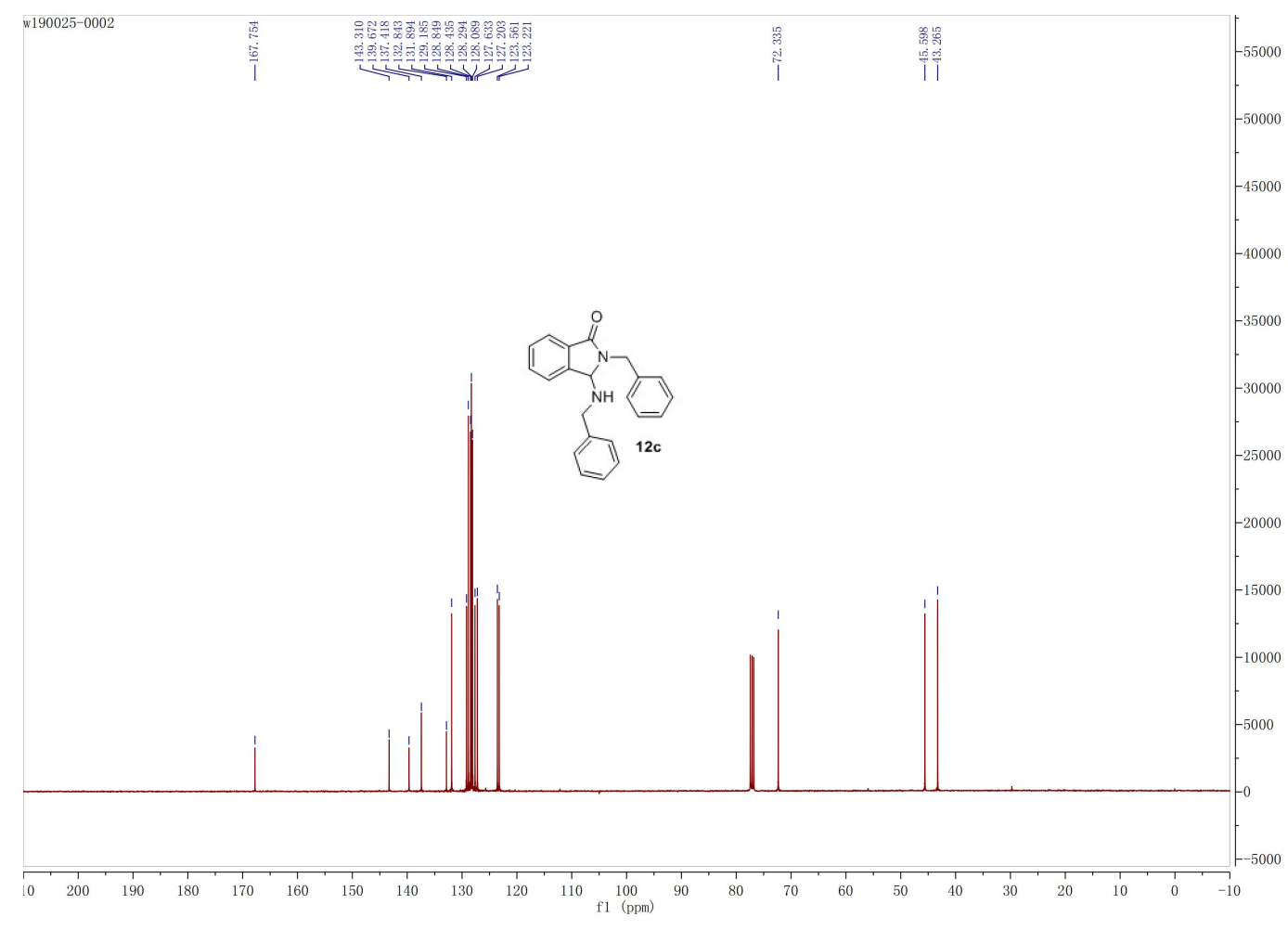

Figure S134. ${ }^{13} \mathrm{CNMR}\left(100 \mathrm{MHz}, \mathrm{CDCl}_{3}\right)$ of compound $12 \mathrm{c}$ 


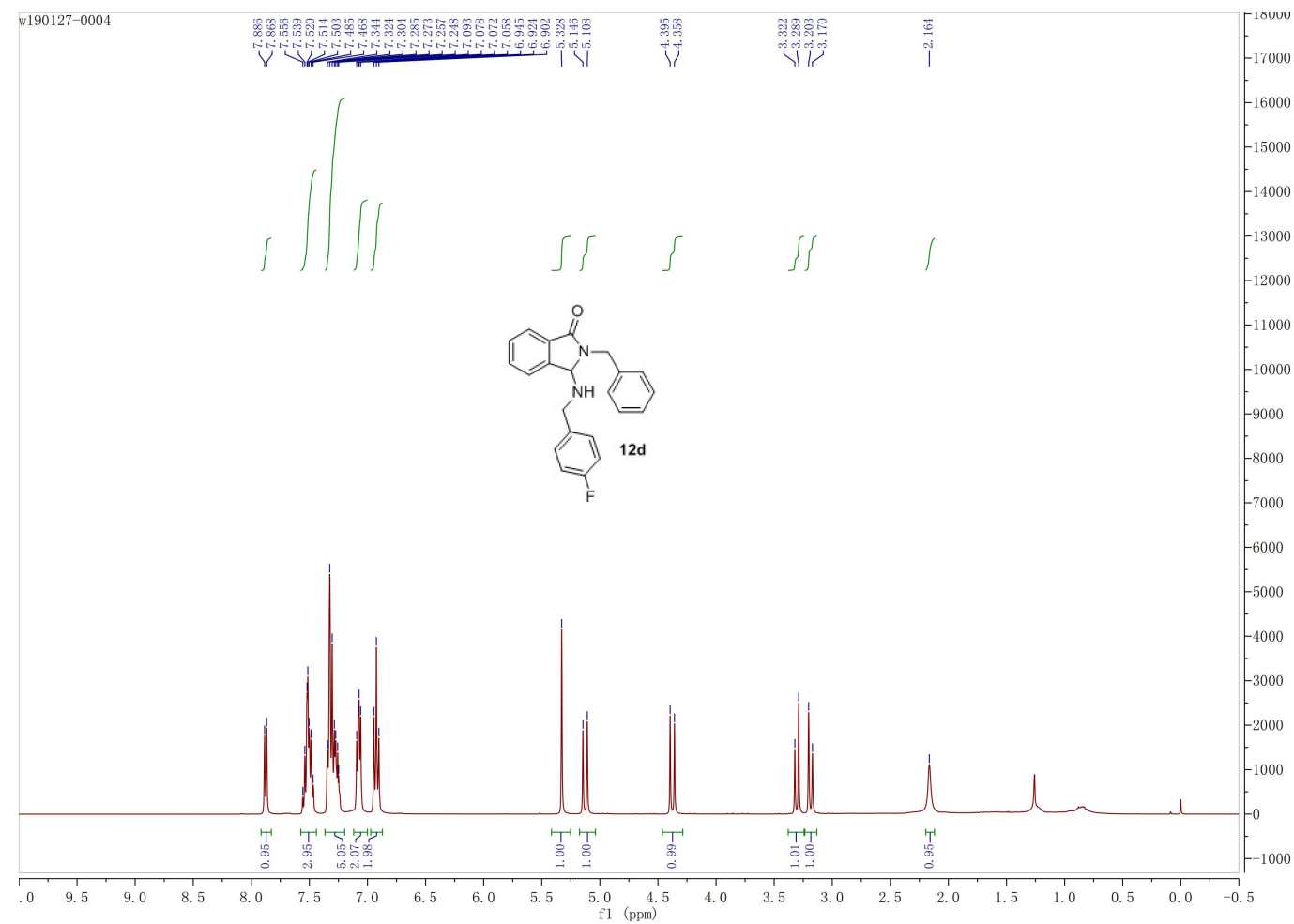

Figure S135. ${ }^{1} \mathrm{HNMR}\left(400 \mathrm{MHz}, \mathrm{CDCl}_{3}\right)$ of compound $12 \mathrm{~d}$

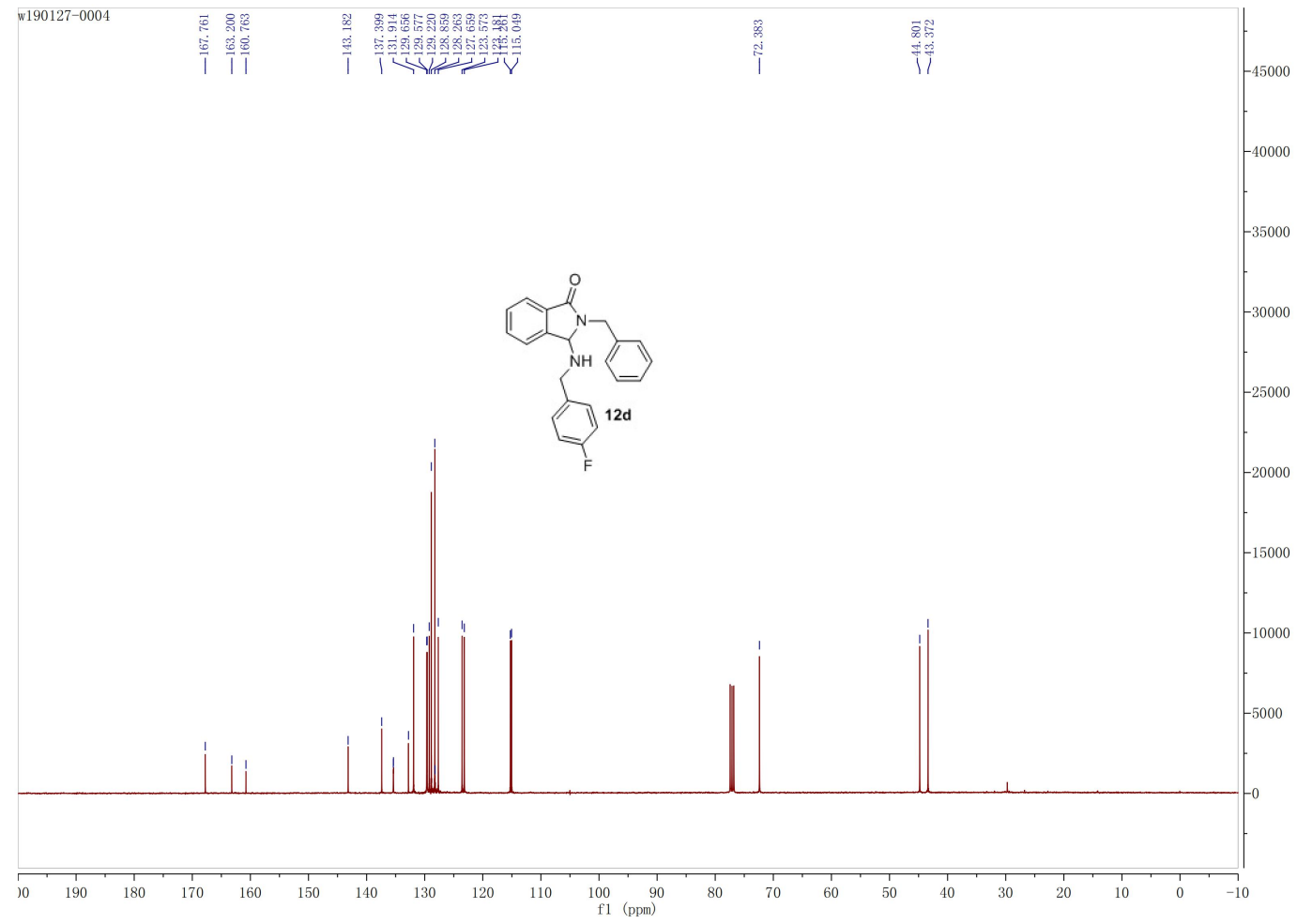

Figure S136. ${ }^{13} \mathrm{CNMR}\left(100 \mathrm{MHz}, \mathrm{CDCl}_{3}\right)$ of compound $12 \mathrm{~d}$ 


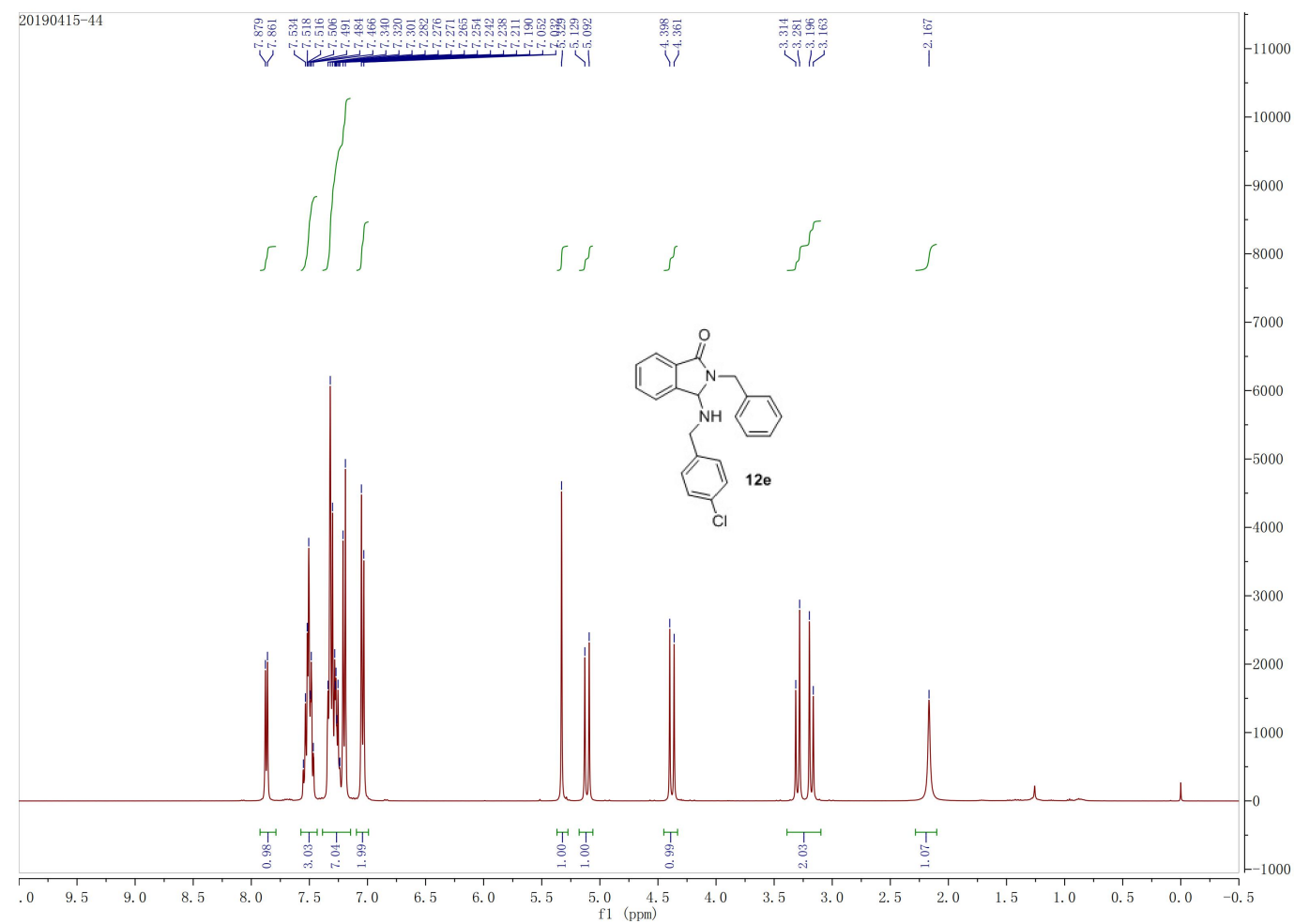

Figure S137. ${ }^{1} \mathrm{HNMR}\left(400 \mathrm{MHz}, \mathrm{CDCl}_{3}\right)$ of compound $12 \mathrm{e}$

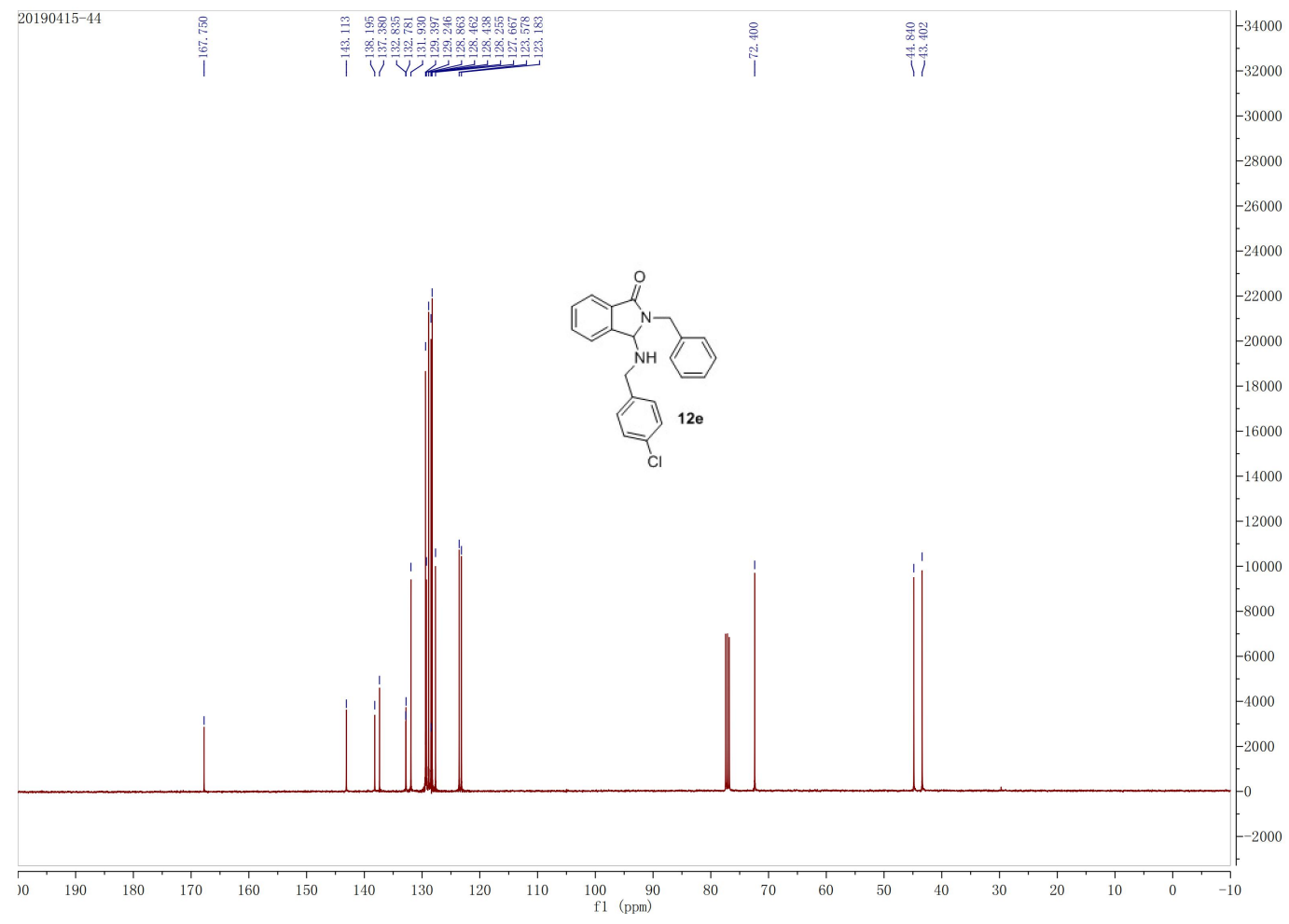

Figure S138. ${ }^{13} \mathrm{CNMR}\left(100 \mathrm{MHz}, \mathrm{CDCl}_{3}\right)$ of compound $12 \mathrm{e}$ 


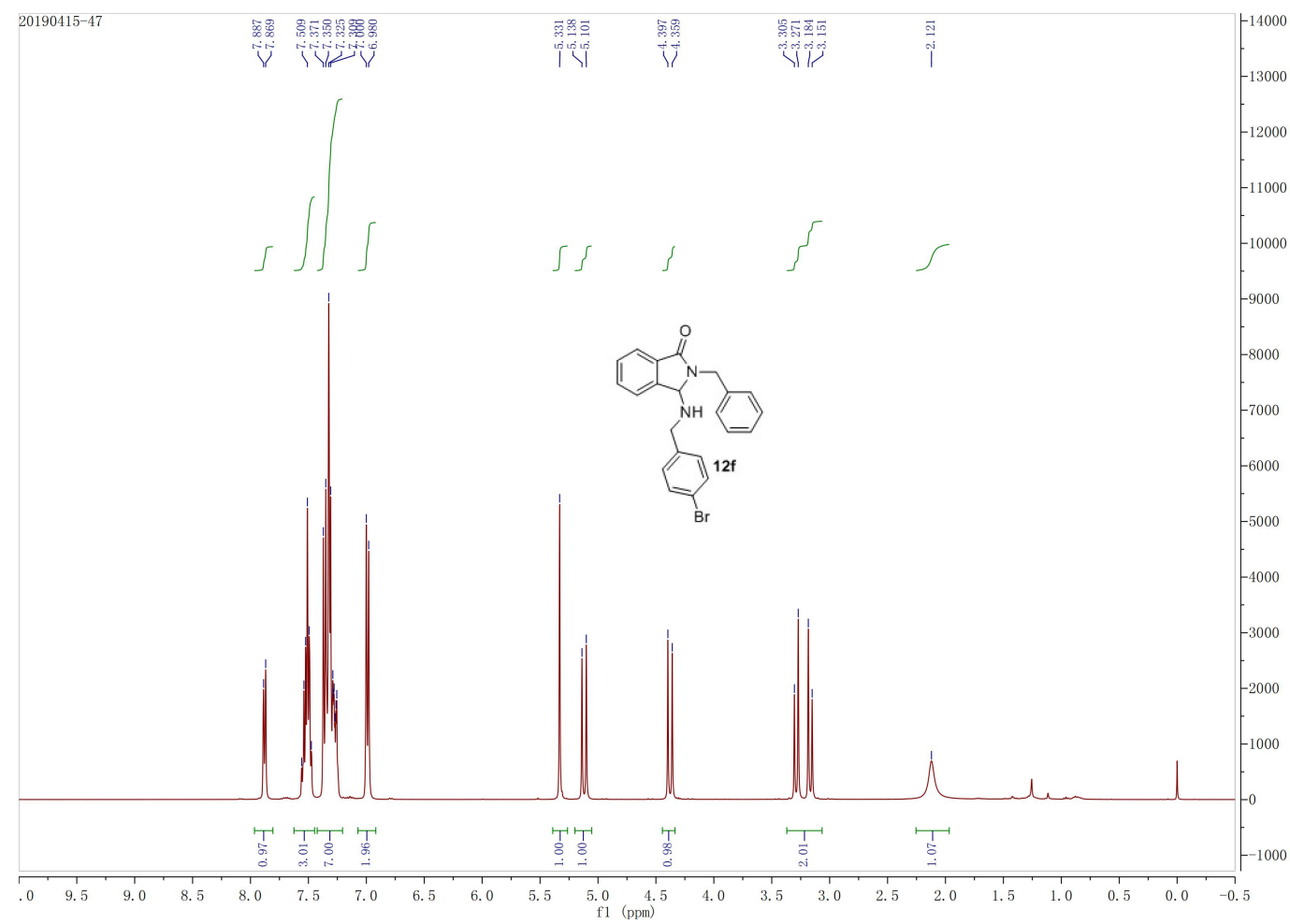

Figure S139. ${ }^{1} \mathrm{HNMR}\left(400 \mathrm{MHz}, \mathrm{CDCl}_{3}\right)$ of compound $12 \mathrm{f}$

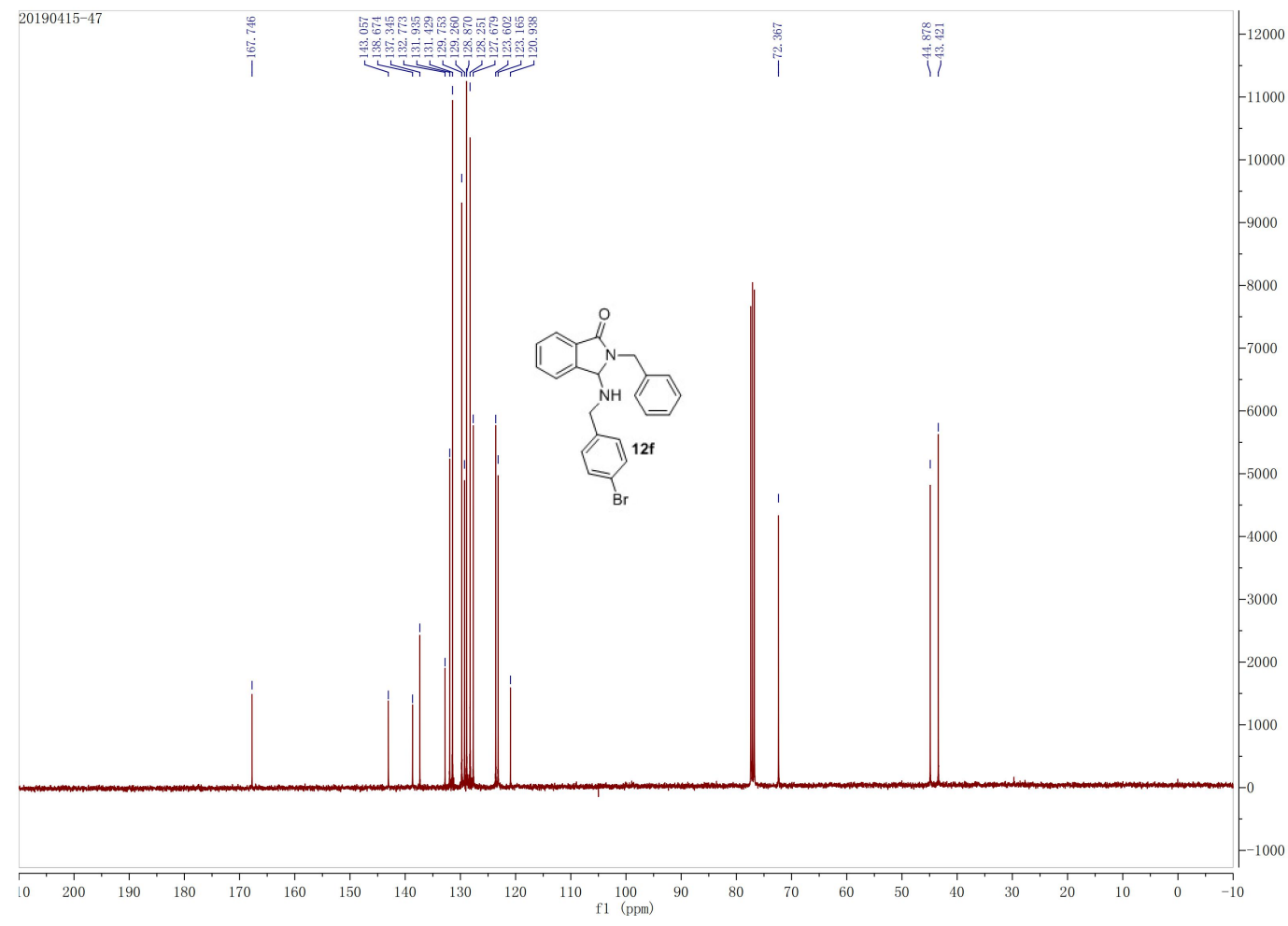

Figure S140. ${ }^{13} \mathrm{CNMR}\left(100 \mathrm{MHz}, \mathrm{CDCl}_{3}\right)$ of compound $12 \mathrm{f}$ 


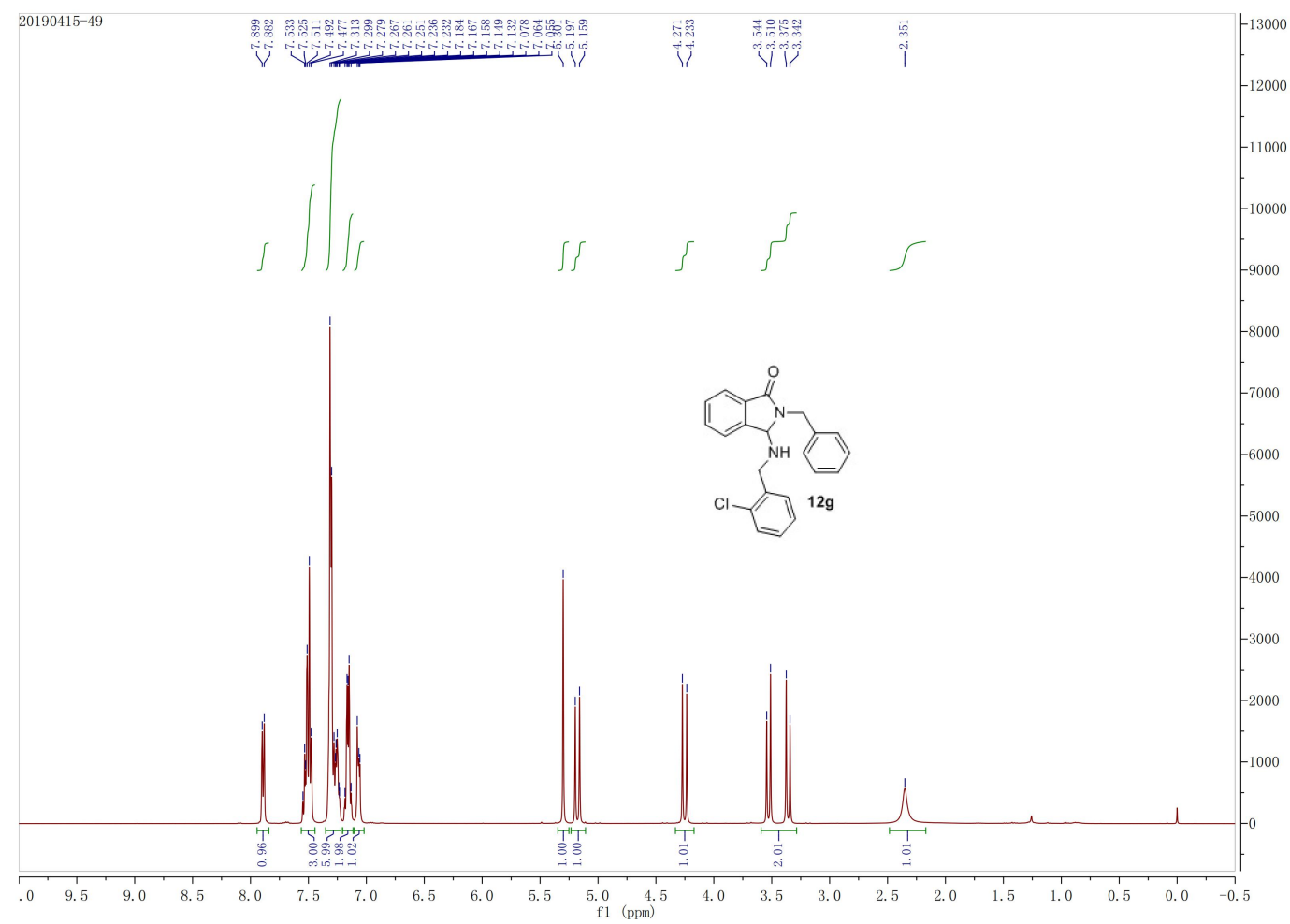

Figure S141. ${ }^{1} \mathrm{HNMR}\left(400 \mathrm{MHz}, \mathrm{CDCl}_{3}\right)$ of compound $12 \mathrm{~g}$

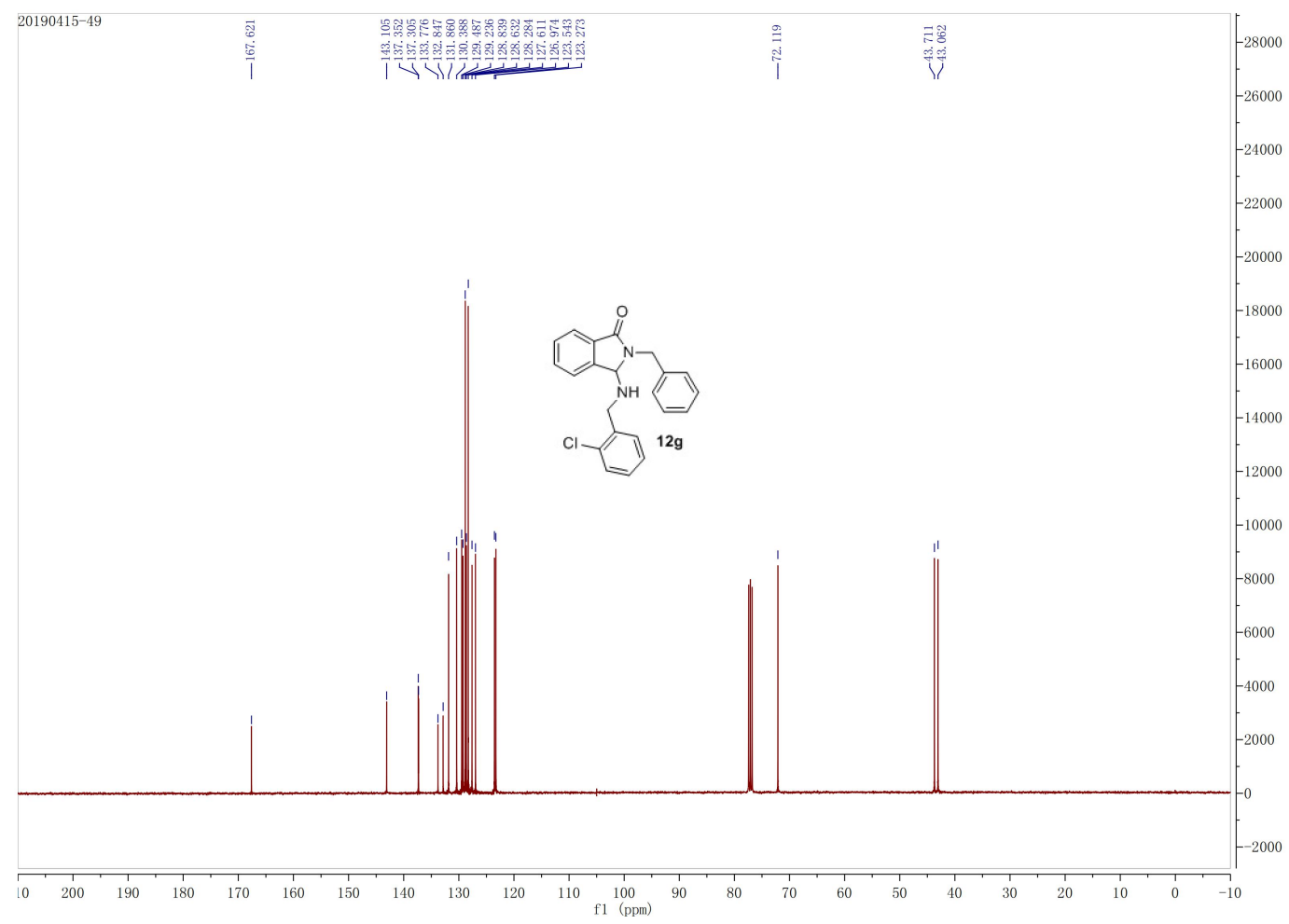

Figure S142. ${ }^{13} \mathrm{CNMR}\left(100 \mathrm{MHz}, \mathrm{CDCl}_{3}\right)$ of compound $12 \mathrm{~g}$ 


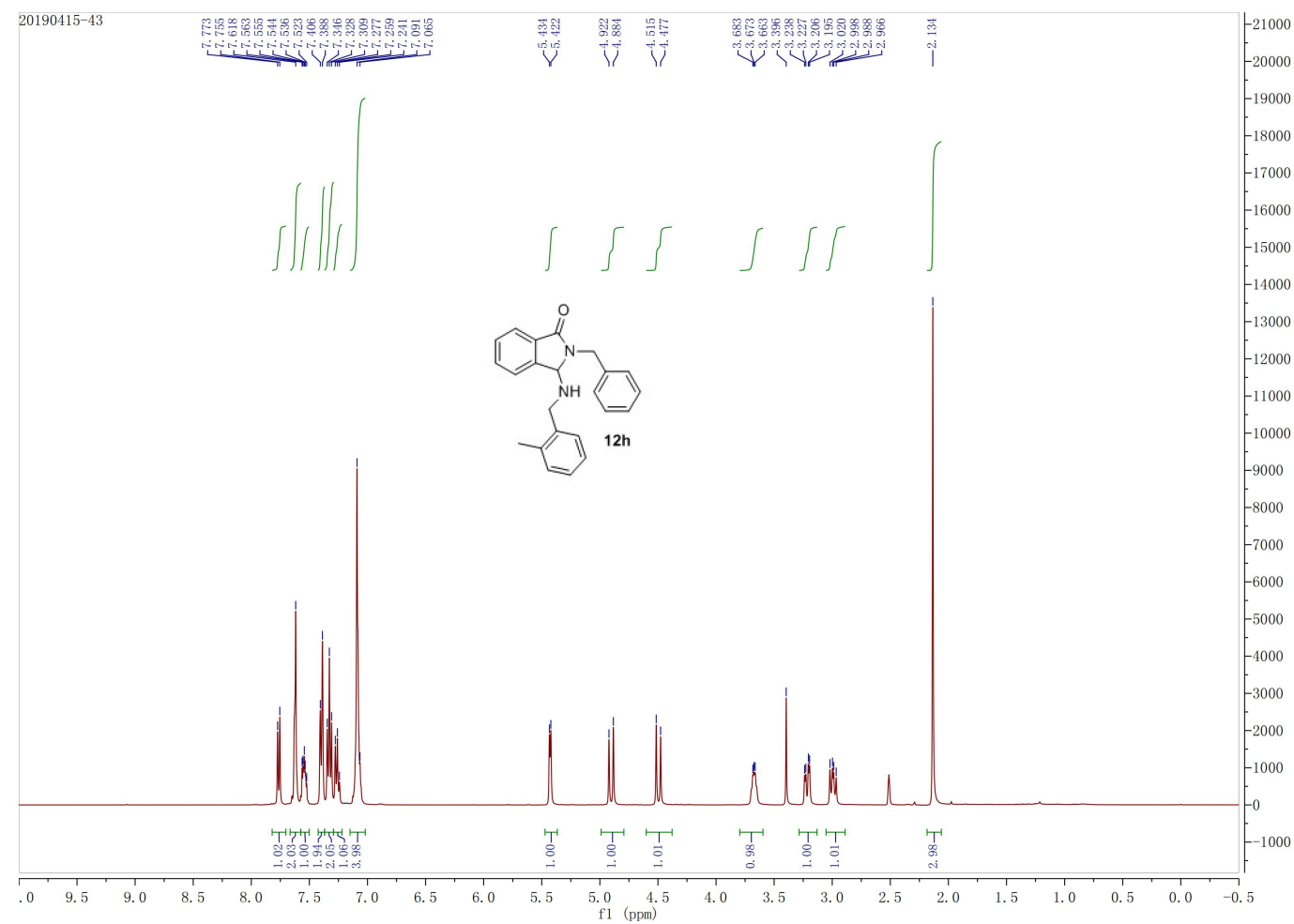

Figure S143. ${ }^{1} \mathrm{HNMR}\left(400 \mathrm{MHz}, \mathrm{CDCl}_{3}\right)$ of compound $12 \mathrm{~h}$

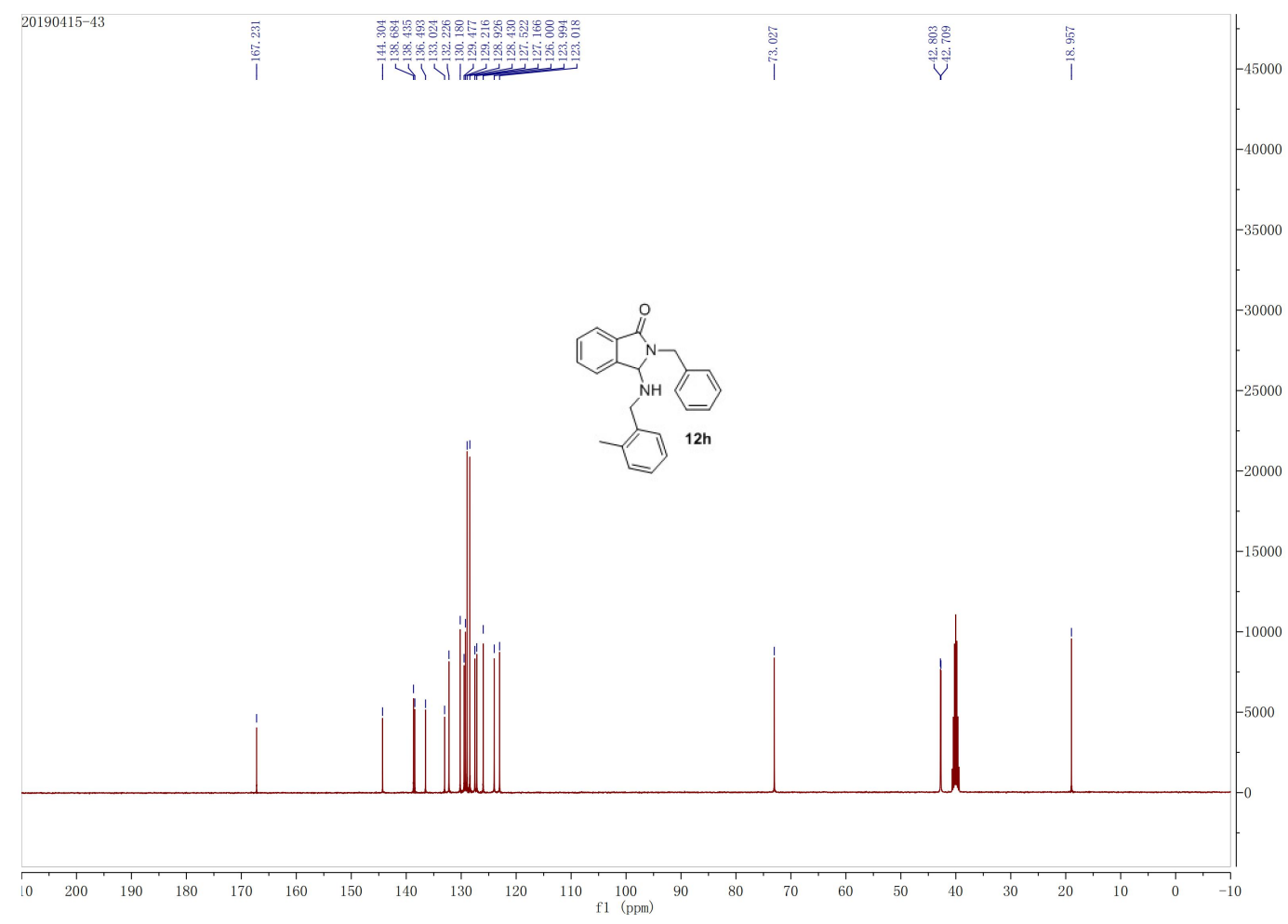

Figure S144. ${ }^{13} \mathrm{CNMR}\left(100 \mathrm{MHz}, \mathrm{CDCl}_{3}\right)$ of compound $12 \mathrm{~h}$ 


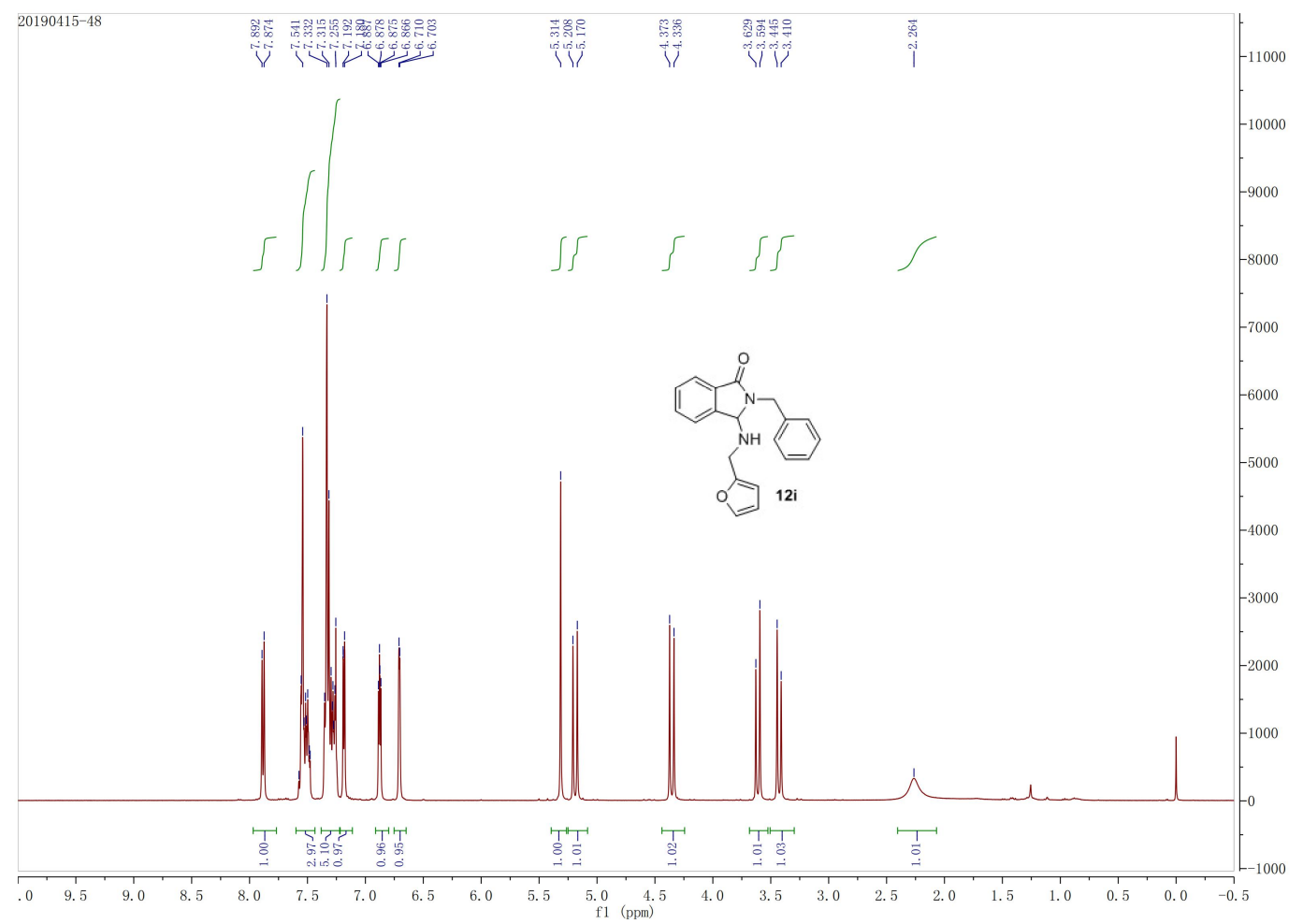

Figure S145. ${ }^{1} \mathrm{HNMR}\left(400 \mathrm{MHz}, \mathrm{CDCl}_{3}\right)$ of compound $12 \mathrm{i}$

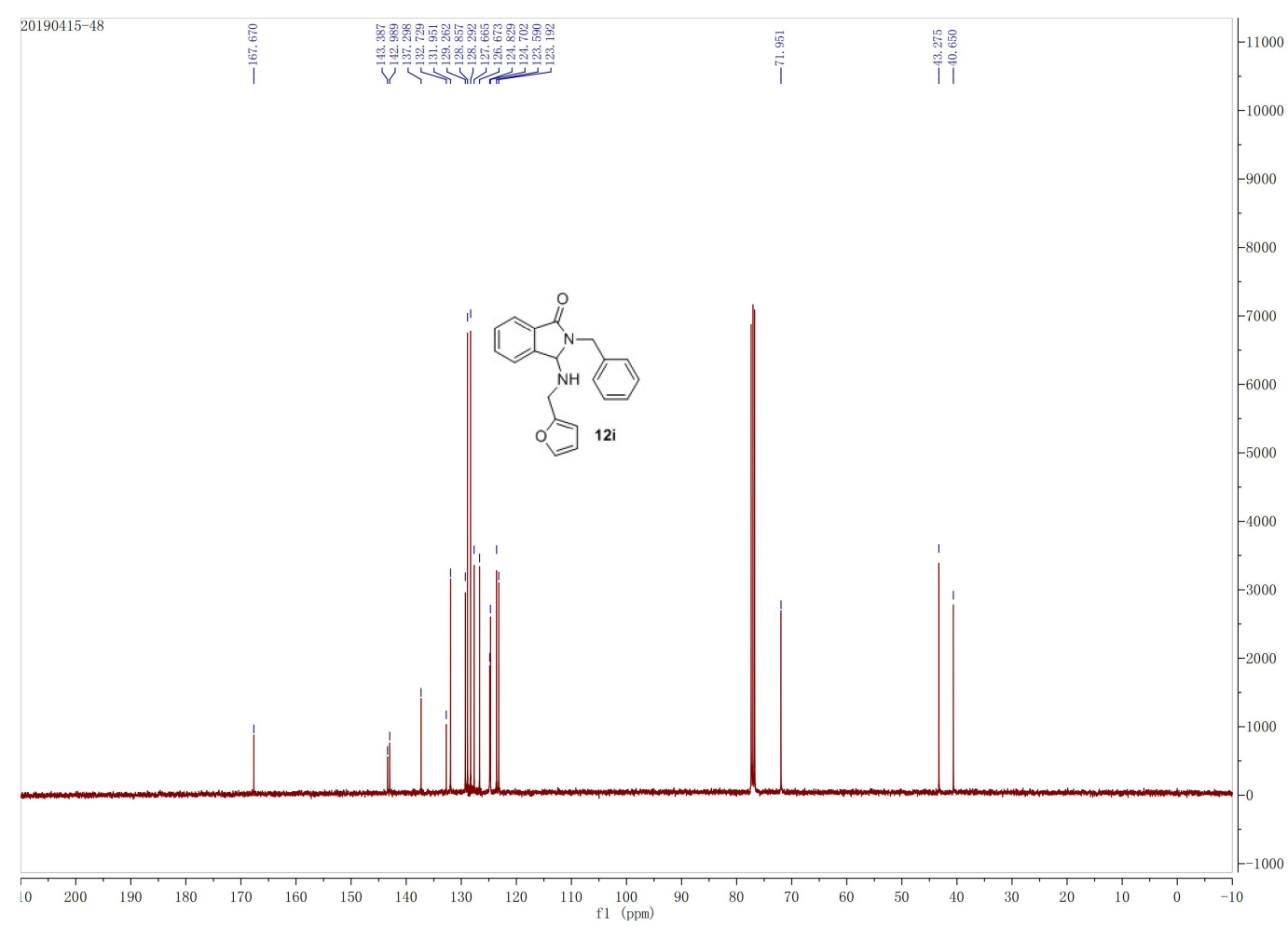

Figure S146. ${ }^{13} \mathrm{CNMR}\left(100 \mathrm{MHz}, \mathrm{CDCl}_{3}\right)$ of compound $12 \mathrm{i}$ 


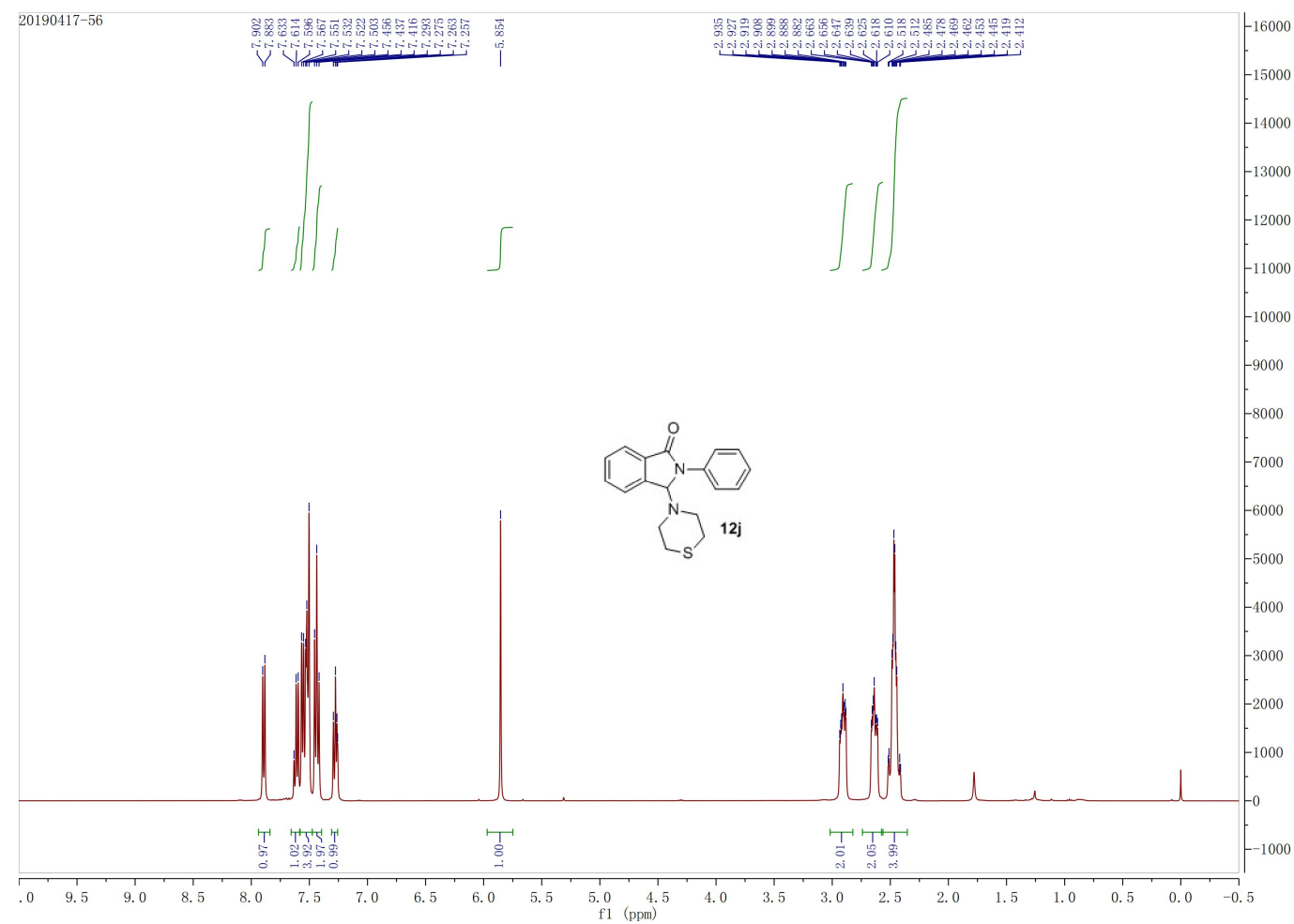

Figure S147. ${ }^{1} \mathrm{HNMR}\left(400 \mathrm{MHz}, \mathrm{CDCl}_{3}\right)$ of compound $12 \mathrm{j}$

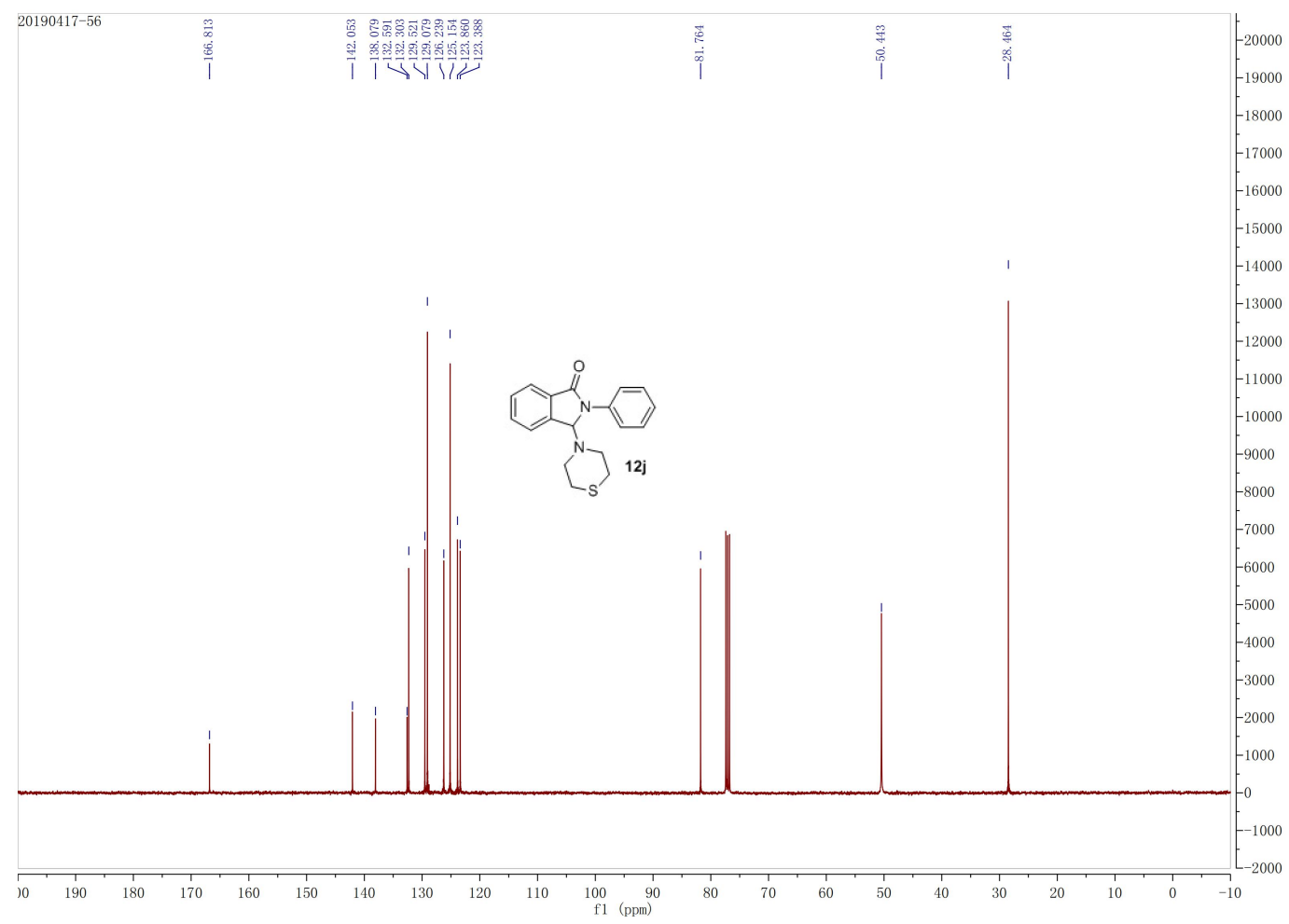

Figure S148. ${ }^{13} \mathrm{CNMR}\left(100 \mathrm{MHz}, \mathrm{CDCl}_{3}\right)$ of compound $12 \mathrm{j}$ 


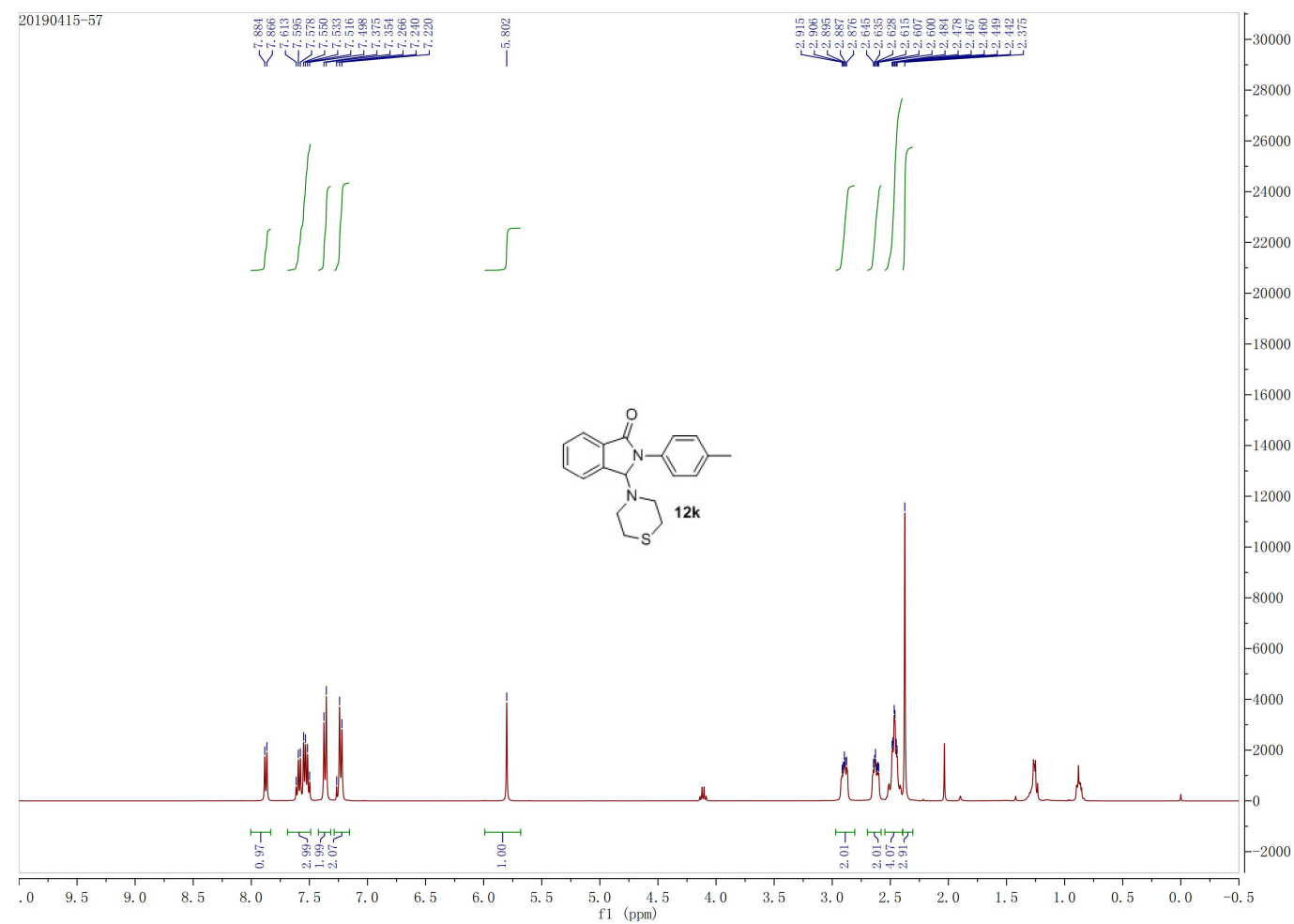

Figure S149. ${ }^{1} \mathrm{HNMR}\left(400 \mathrm{MHz}, \mathrm{CDCl}_{3}\right)$ of compound $12 \mathrm{k}$

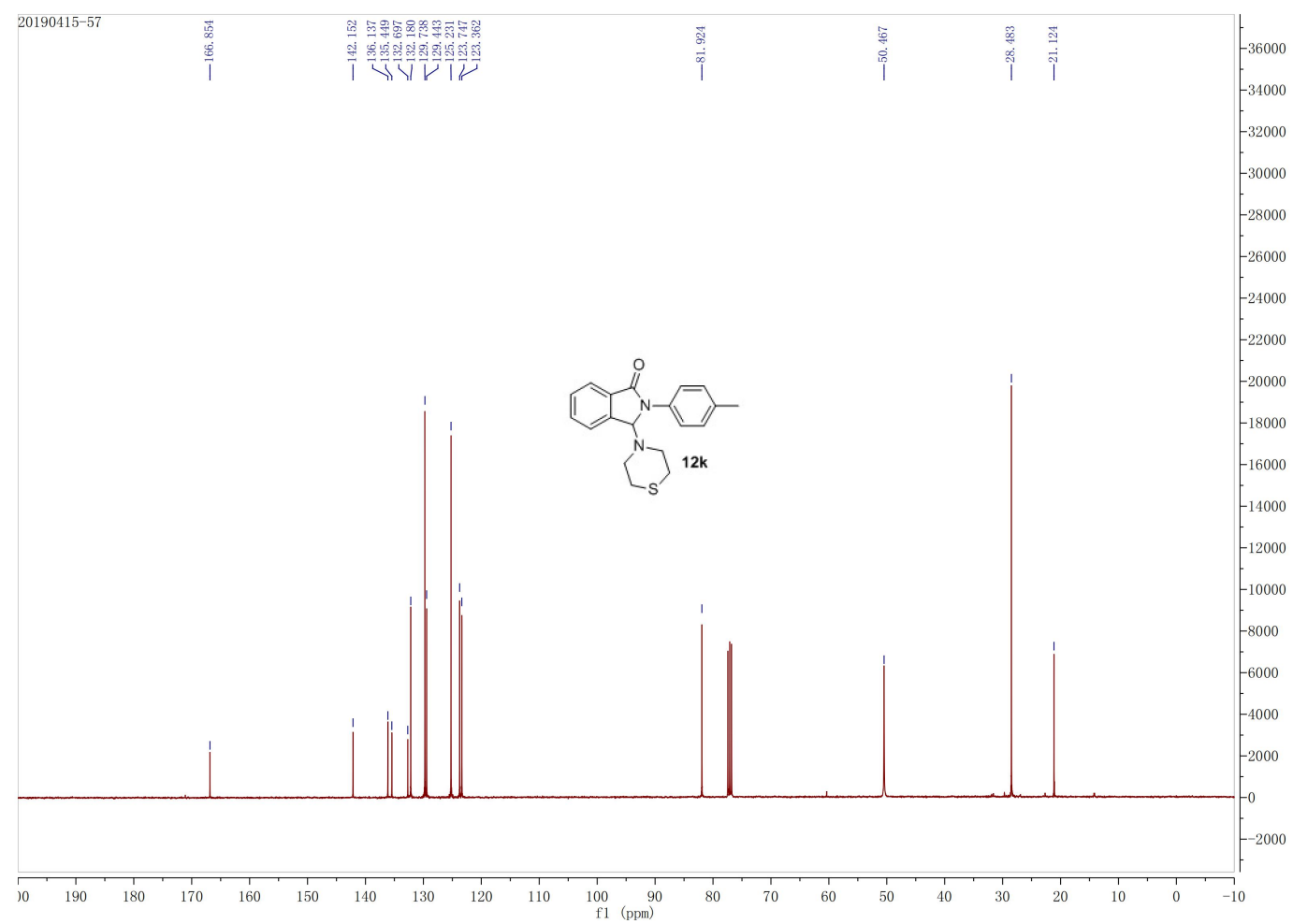

Figure S150. ${ }^{13} \mathrm{CNMR}\left(100 \mathrm{MHz}, \mathrm{CDCl}_{3}\right)$ of compound $12 \mathrm{k}$ 


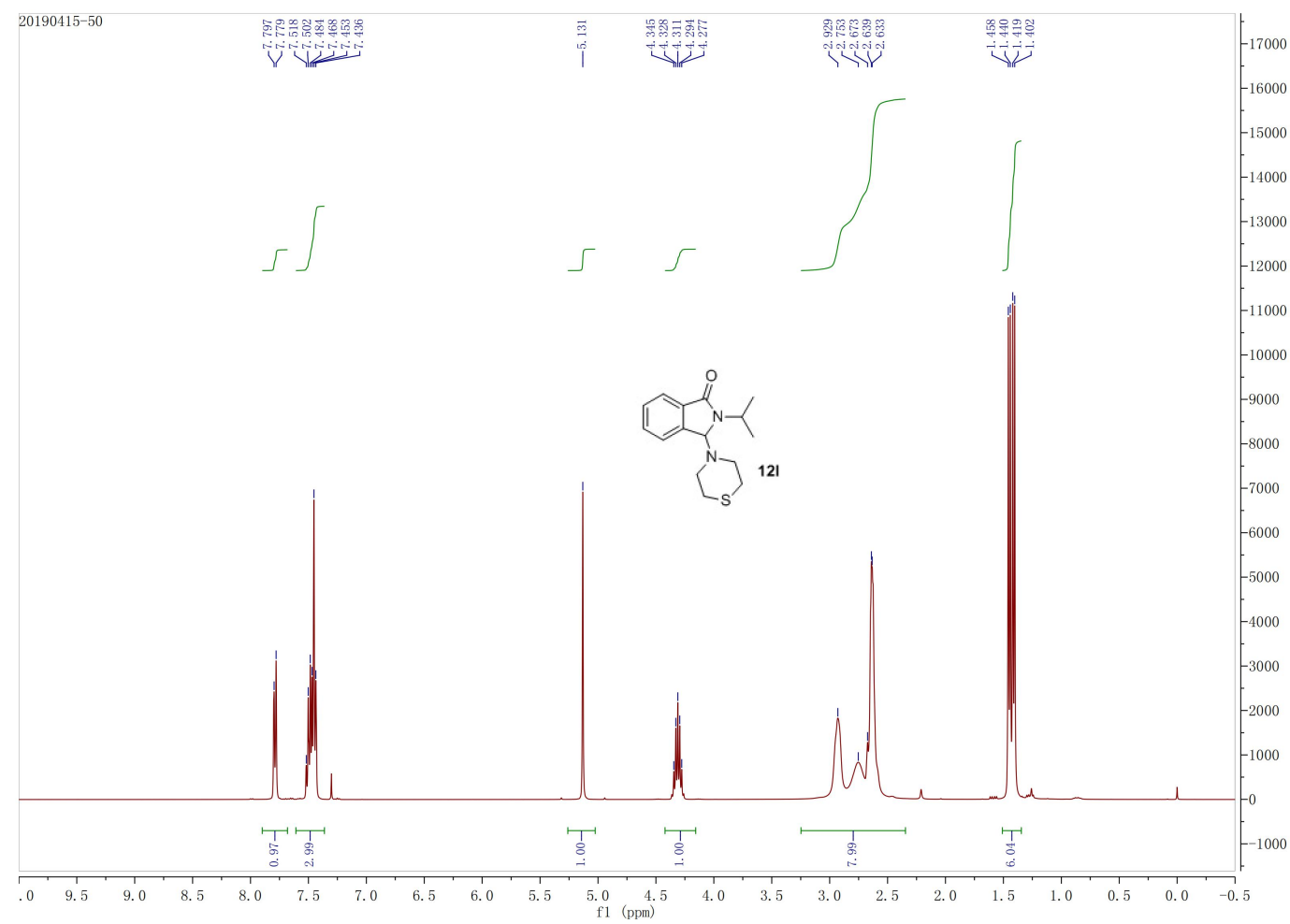

Figure S151. ${ }^{1} \mathrm{HNMR}\left(400 \mathrm{MHz}, \mathrm{CDCl}_{3}\right)$ of compound $12 \mathrm{I}$

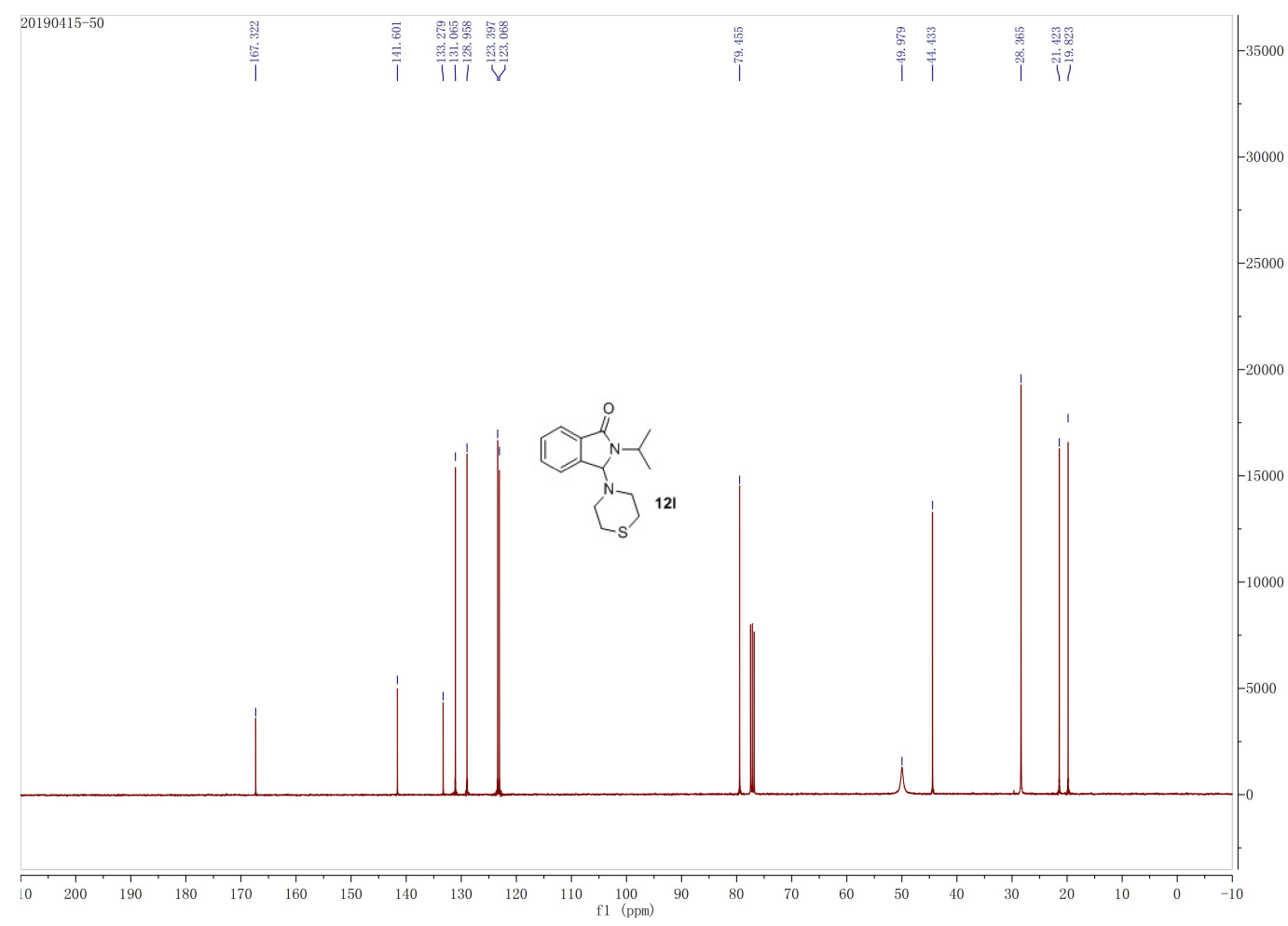

Figure S152. ${ }^{13} \mathrm{CNMR}\left(100 \mathrm{MHz}, \mathrm{CDCl}_{3}\right)$ of compound $12 \mathrm{I}$ 


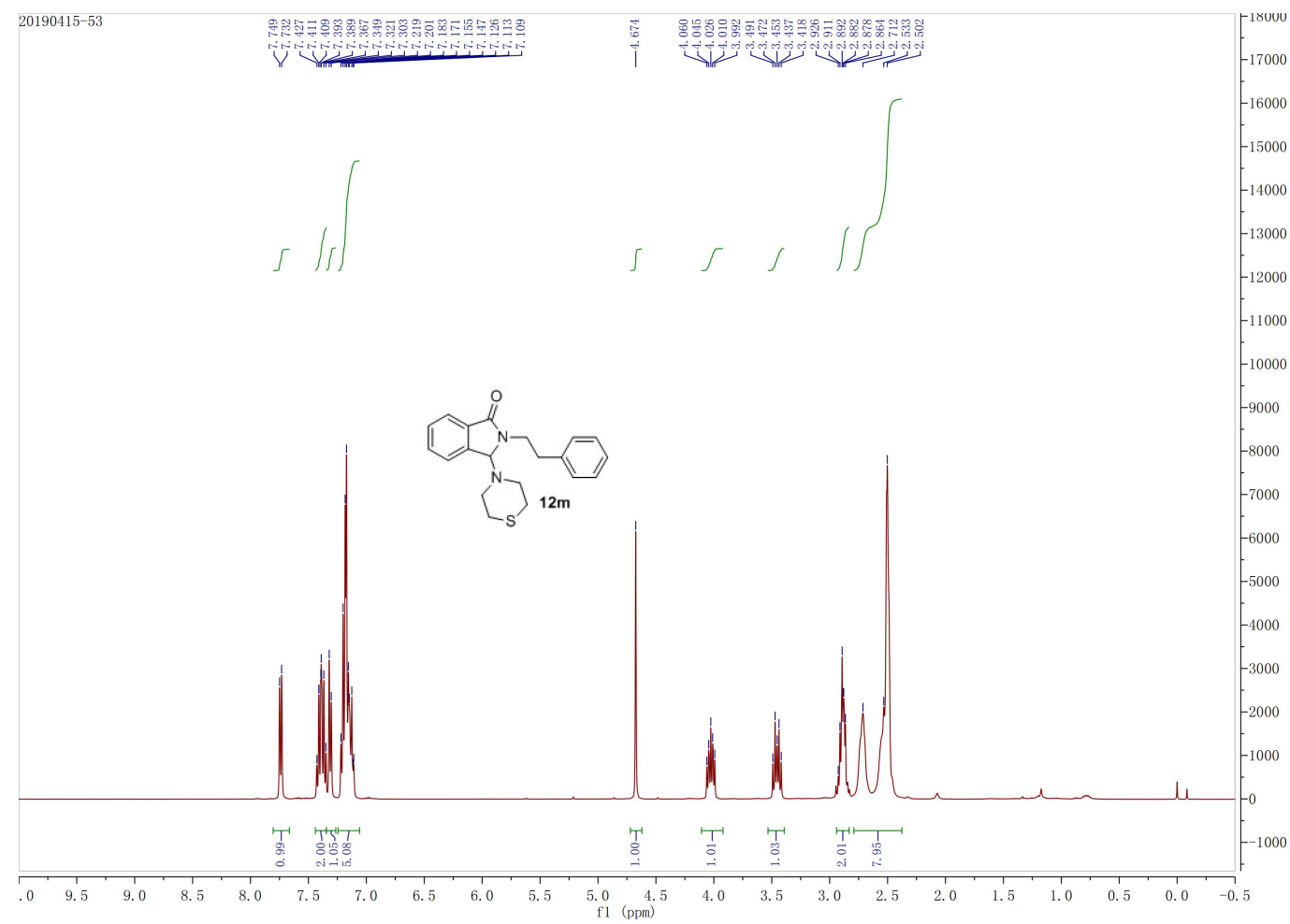

Figure S153. ${ }^{1} \mathrm{HNMR}\left(400 \mathrm{MHz}, \mathrm{CDCl}_{3}\right)$ of compound $12 \mathrm{~m}$

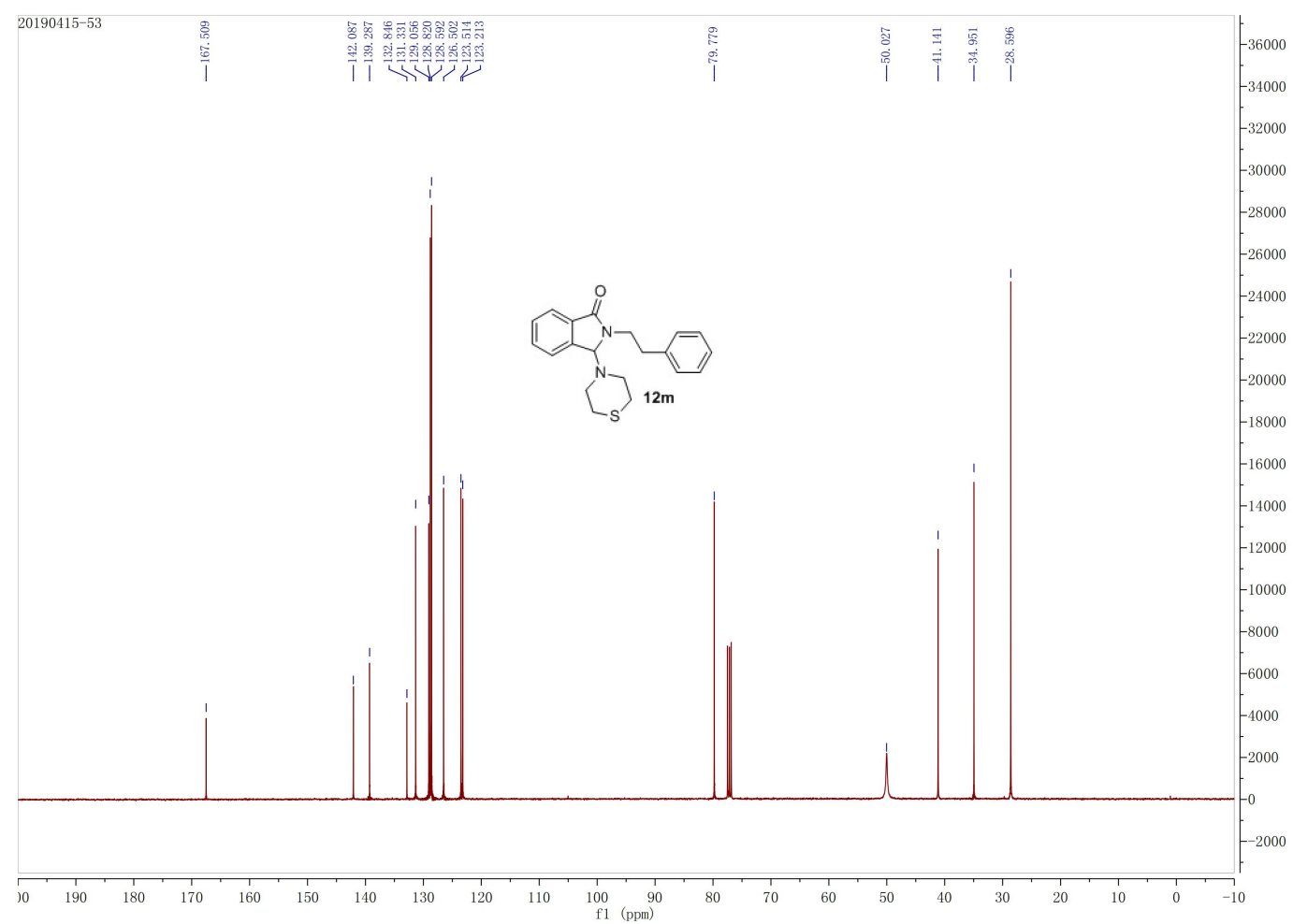

Figure S154. ${ }^{13} \mathrm{CNMR}\left(100 \mathrm{MHz}, \mathrm{CDCl}_{3}\right)$ of compound $12 \mathrm{~m}$ 


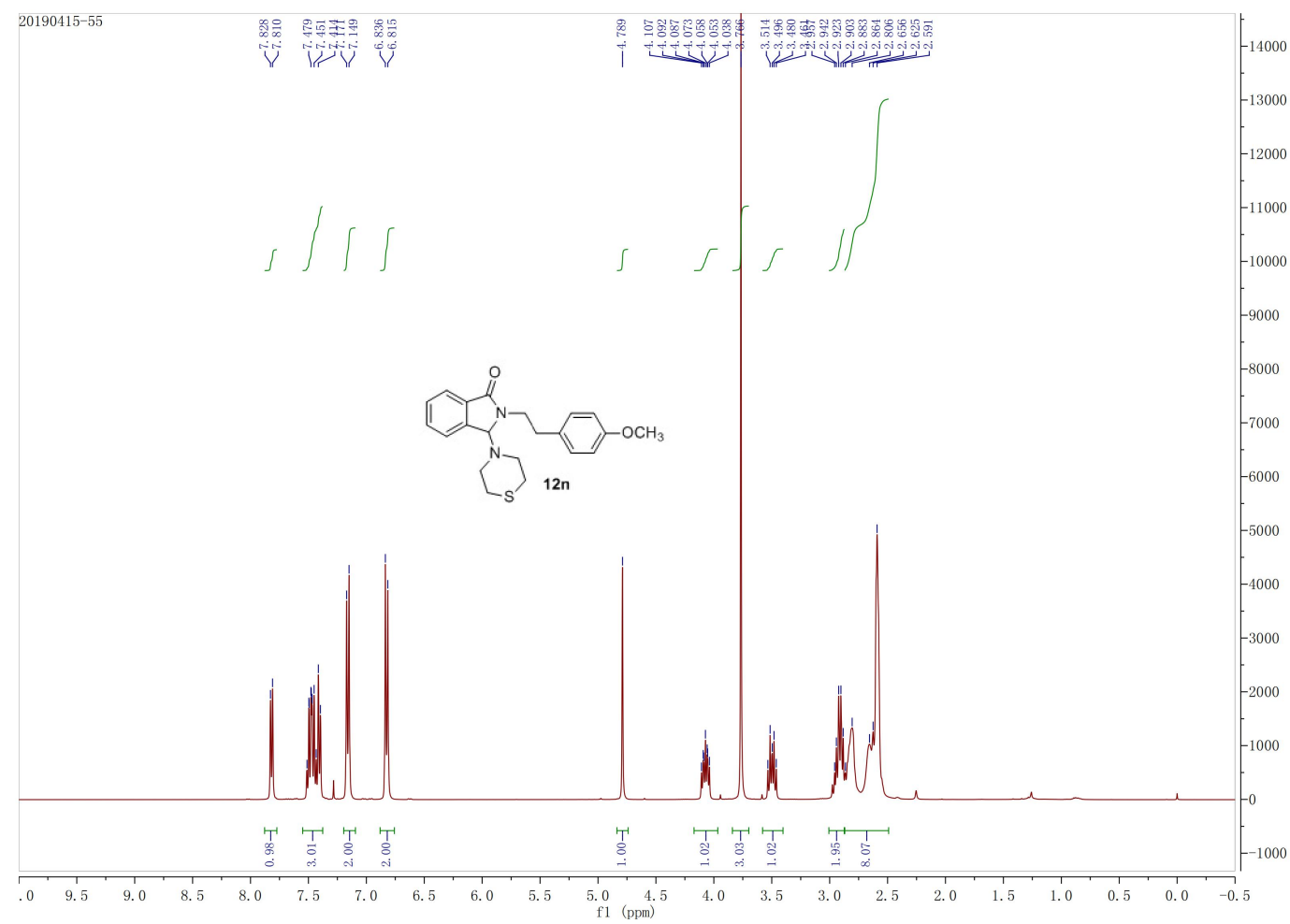

Figure S155. ${ }^{1} \mathrm{HNMR}\left(400 \mathrm{MHz}, \mathrm{CDCl}_{3}\right)$ of compound $12 \mathrm{n}$

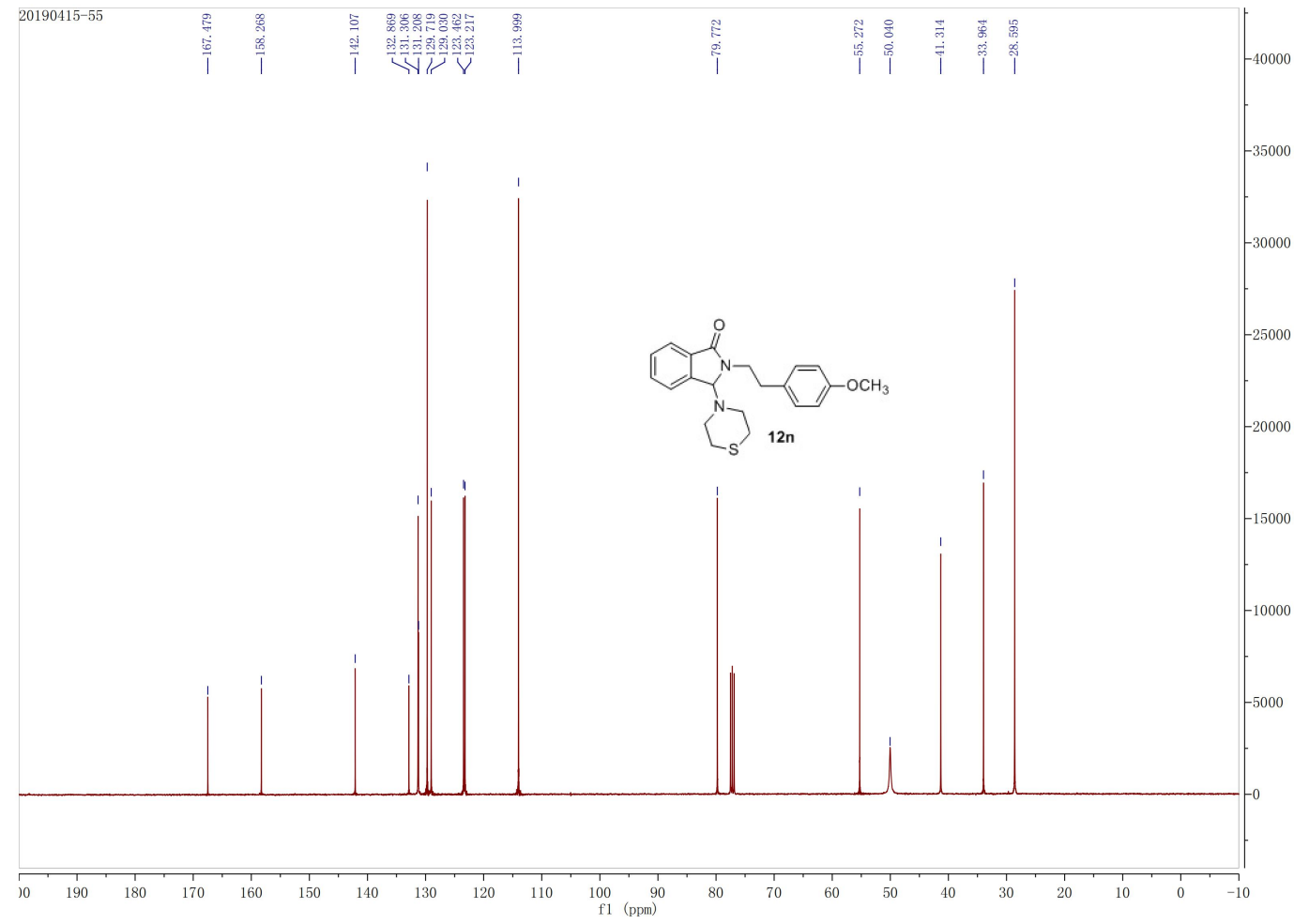

Figure S156. ${ }^{13} \mathrm{CNMR}\left(100 \mathrm{MHz}, \mathrm{CDCl}_{3}\right)$ of compound $12 \mathrm{n}$ 


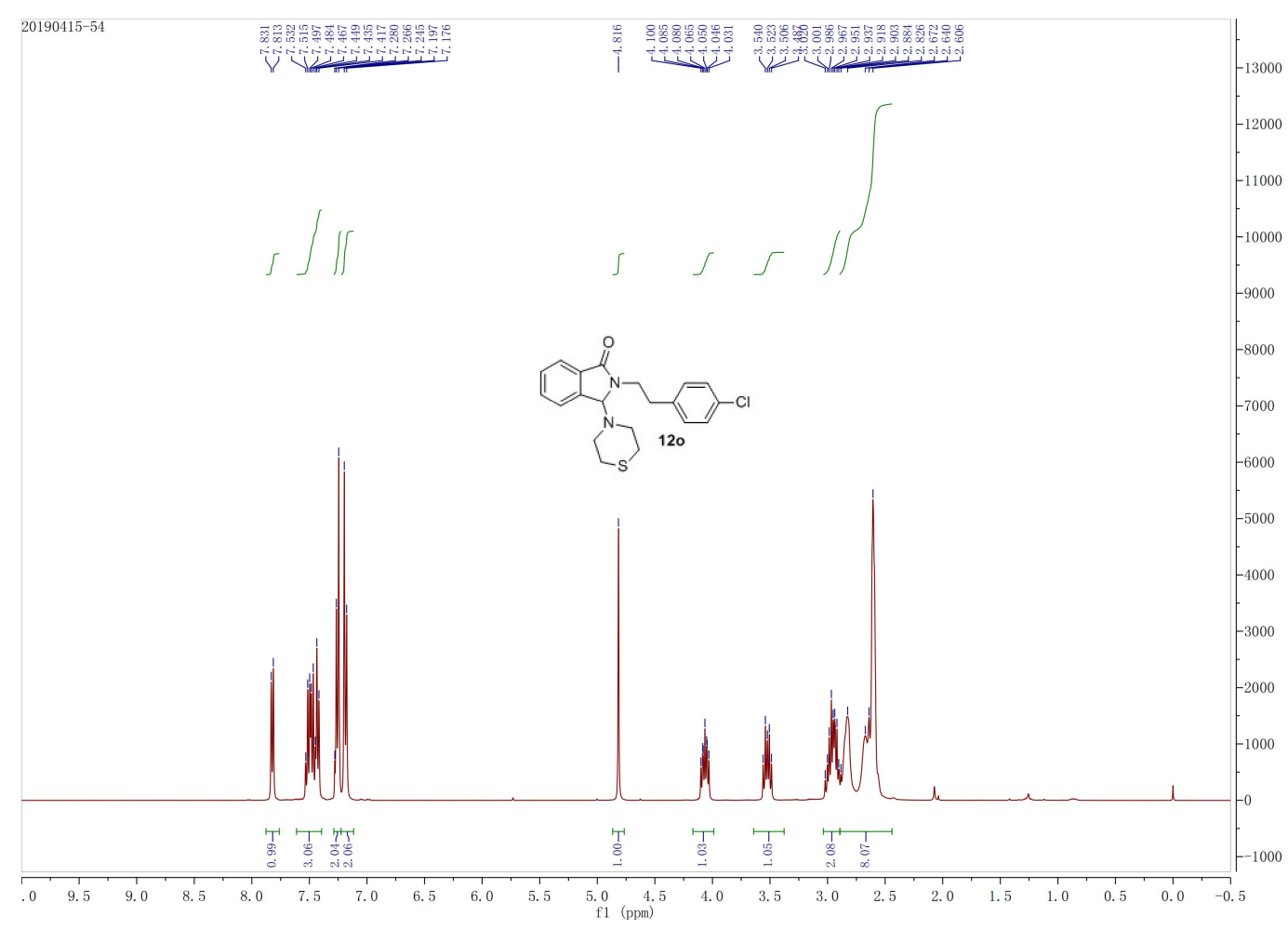

Figure S157. ${ }^{1} \mathrm{HNMR}\left(400 \mathrm{MHz}, \mathrm{CDCl}_{3}\right)$ of compound $12 \mathrm{o}$

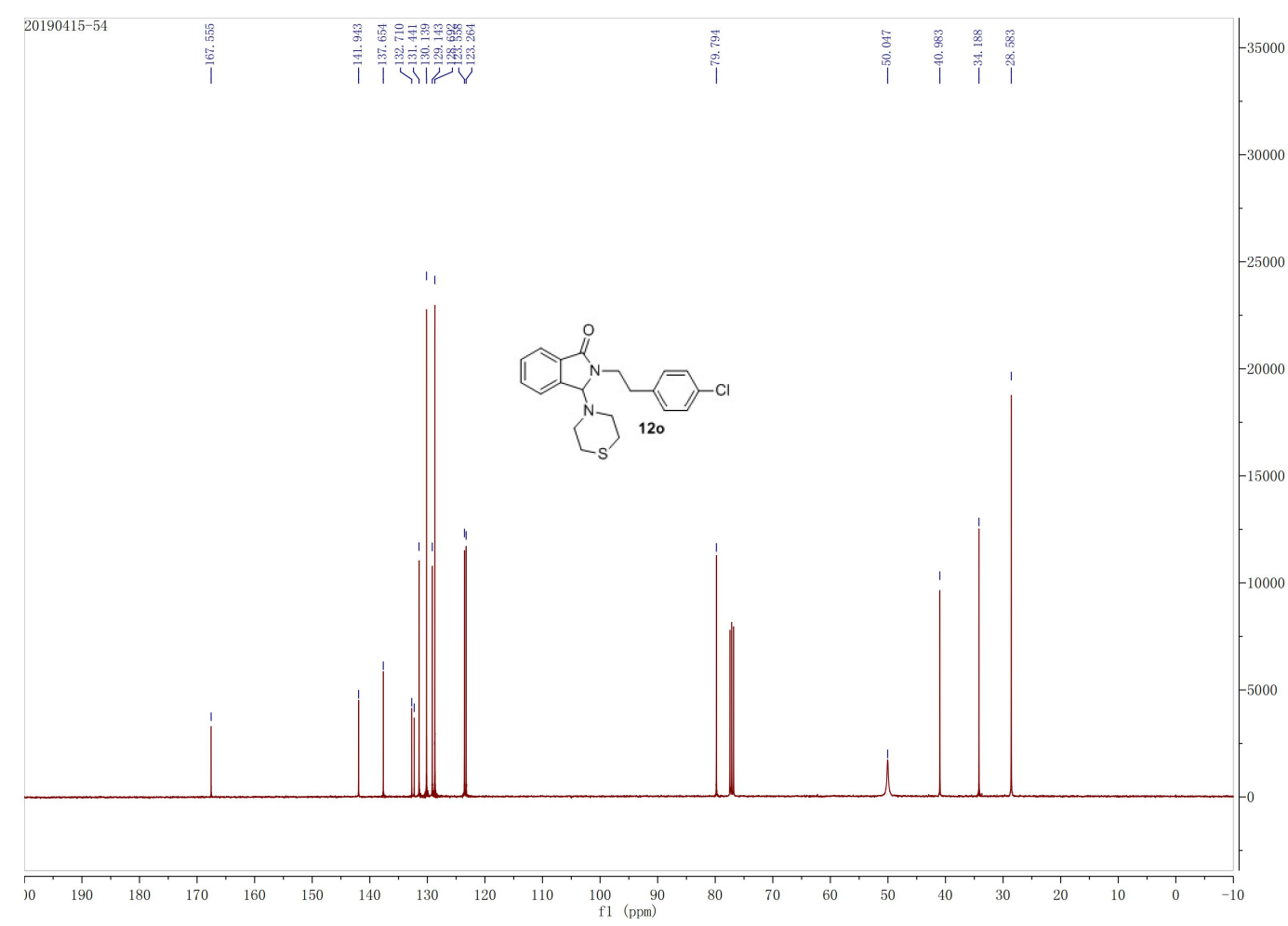

Figure S158. ${ }^{13} \mathrm{CNMR}\left(100 \mathrm{MHz}, \mathrm{CDCl}_{3}\right)$ of compound 120 


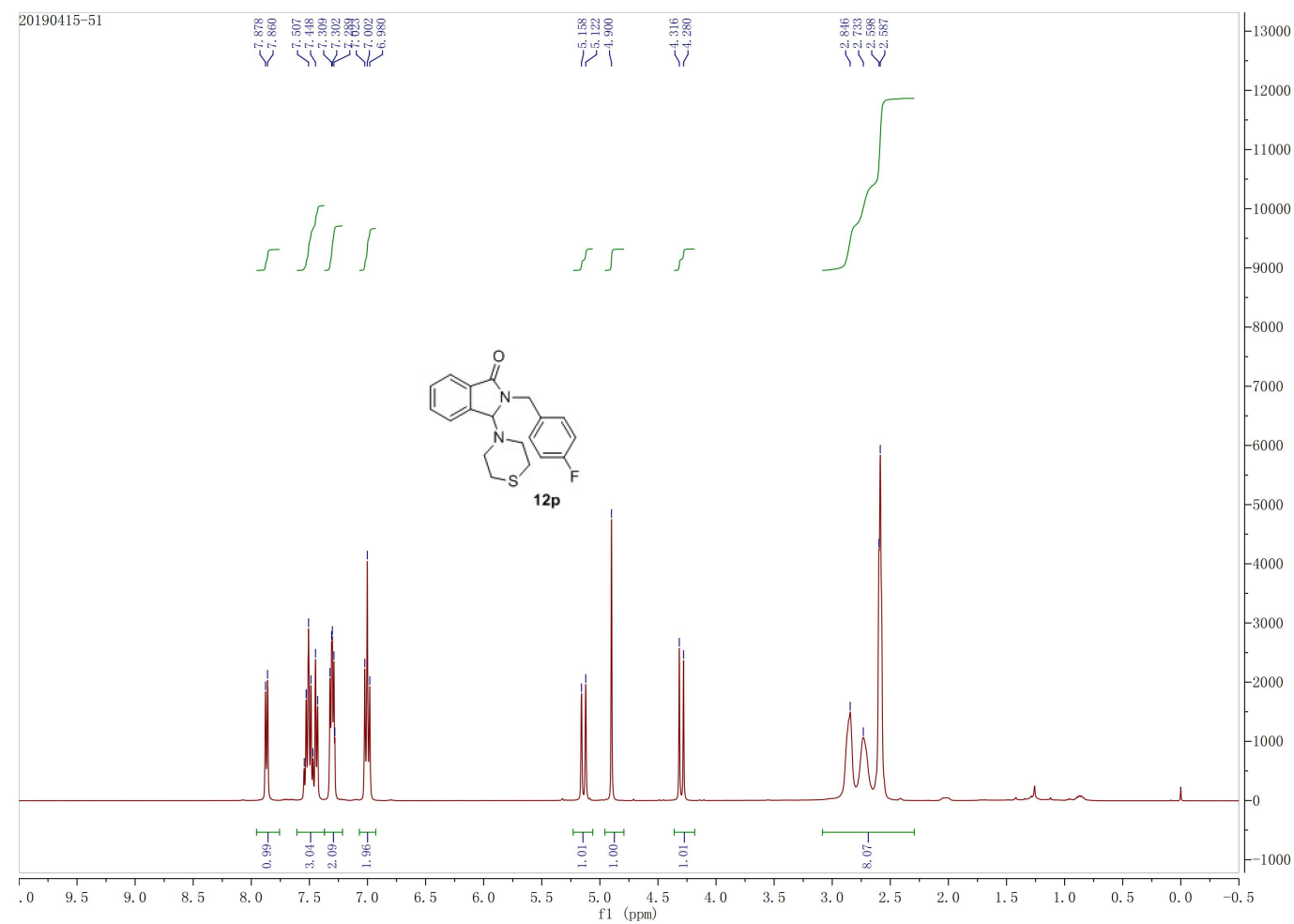

Figure S159. ${ }^{1} \mathrm{HNMR}\left(400 \mathrm{MHz}, \mathrm{CDCl}_{3}\right)$ of compound $12 \mathrm{p}$

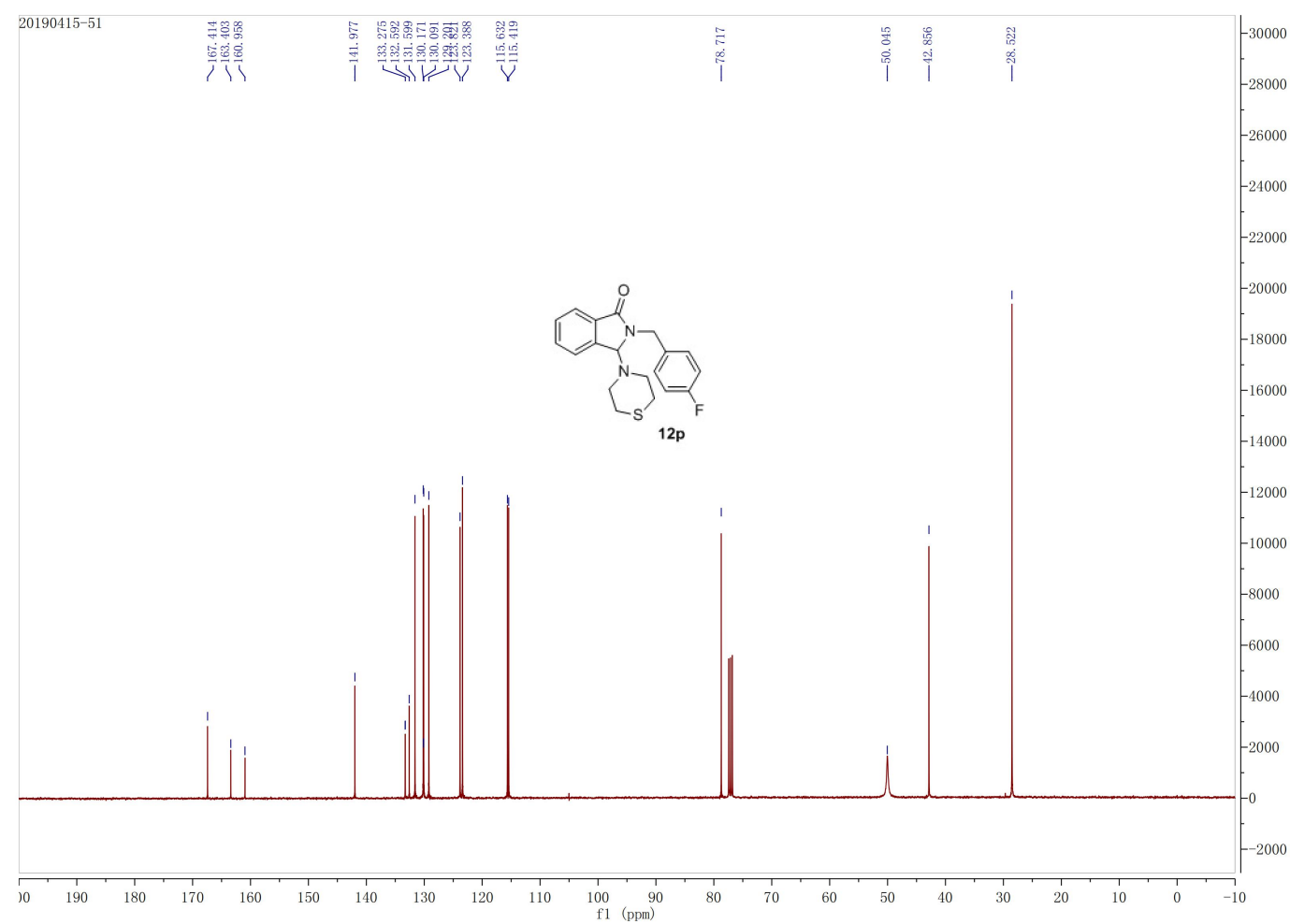

Figure S160. ${ }^{13} \mathrm{CNMR}\left(100 \mathrm{MHz}, \mathrm{CDCl}_{3}\right)$ of compound $12 \mathrm{p}$ 


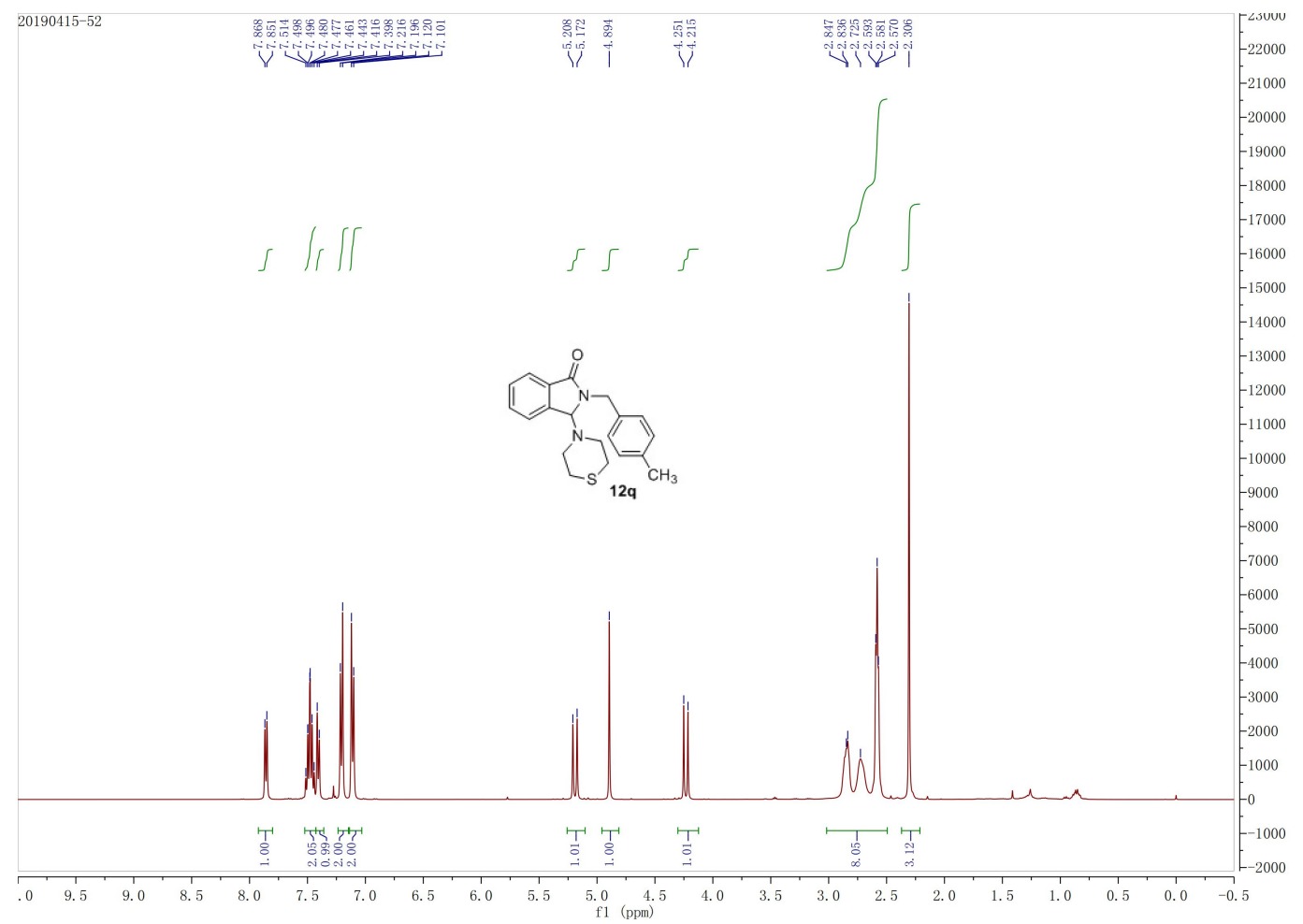

Figure S161. ${ }^{1} \mathrm{HNMR}\left(400 \mathrm{MHz}, \mathrm{CDCl}_{3}\right)$ of compound $12 \mathrm{q}$

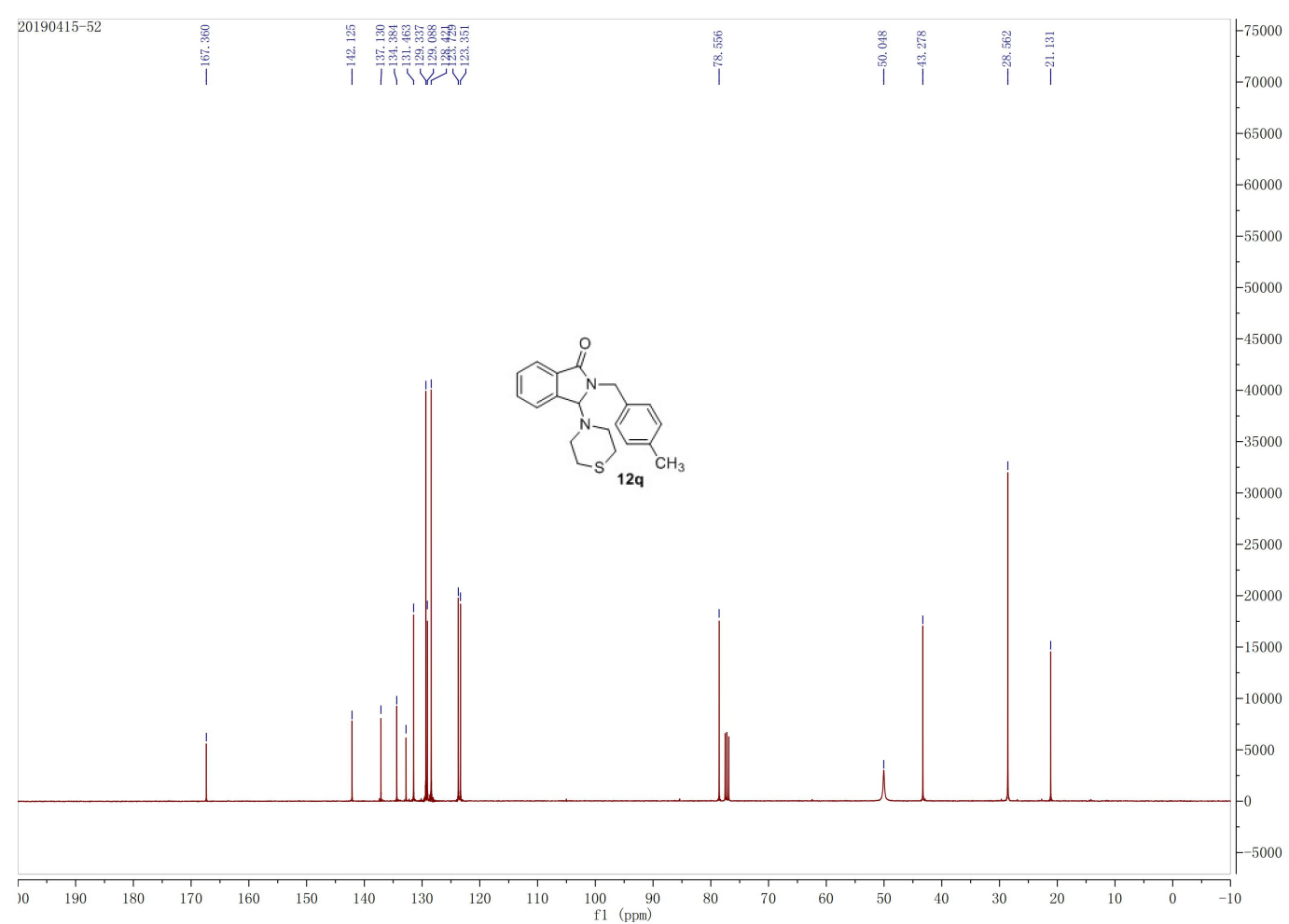

Figure S162. ${ }^{13} \mathrm{CNMR}\left(100 \mathrm{MHz}, \mathrm{CDCl}_{3}\right)$ of compound $12 \mathrm{q}$ 